FINAL REPORT

SUBCONTRACT NO. 9-L68-0562R-1

\title{
CAVITATION AND TWO-PHASE FLOW CHARACTERISTICS OF SRPR (SAVANNAH RIVER PLANT REACTOR) PUMP
}

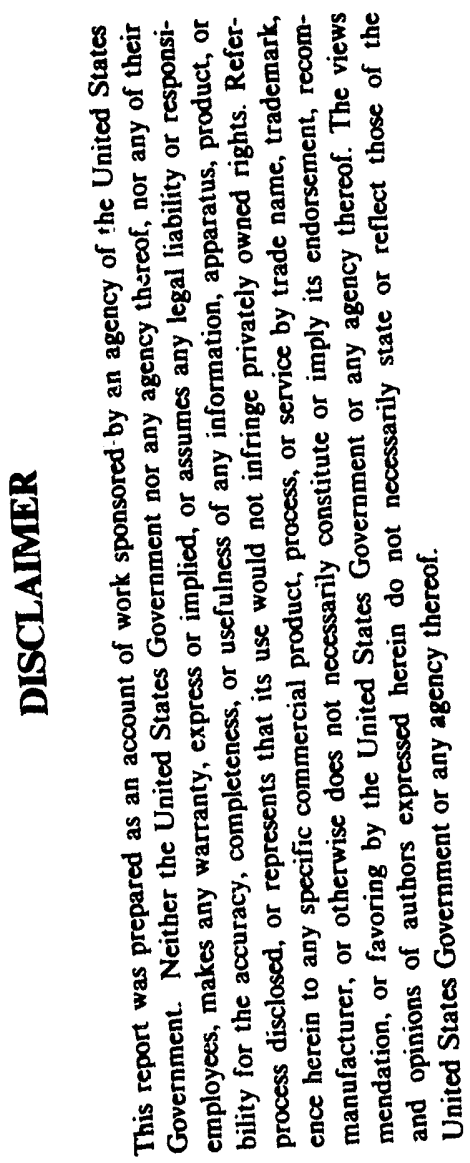

Prepared for

UNIVERSITY OF CALIFORNIA

LOS ALAMOS NATIONAL LABORATORY

LOS ALAMOS, NM 87545

Prepared by

OF TECHNOLOGIES, INC.

1365 RIVIERA DRIVE

PASADENA, CA 91107

JULY 1991

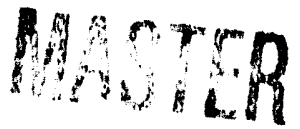


The possible head degradation of the SRPR pumps may be attributable to two independent phenomena, one due to the inception of cavitation and the other due to the two-phase flow phenomena. The head degradation due to the appearance of cavitation on the pump blade is hardly likely in the conventional pressurized water reactor (PWR) since the coolant circulating line is highly pressurized so that the cavitation is difficult to occur even at LOCA (loss of coolant accident) conditions. (Note that the line pressure at PWR is as high as 2500 psi., see the paper of Furuya(1985)).) On the other hand, the suction pressure of SRPR pump is order-of-magnitude smaller than that of PWR so that the cavitation phenomena may prevail, should LOCA occur, depending on the extent of LOCA condition. In this study, therefore, both cavitation phenomena and two-phase flow phenomena were investigated for the SRPR pump by using various analytical tools and the numerical results are presented herein.
\end{abstract}


TABLE OF CONTENTS

1. Introduction. $1-1$

2. Cavitating Flow Performance of SRPR (Savannah River Plant Reactor)

Pumps (Tasks 2, 6, 13 and 14)).

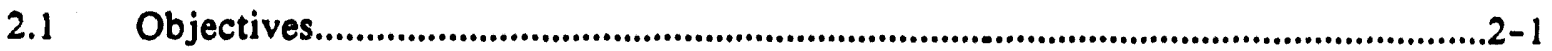

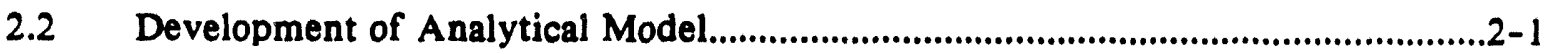

2.2.1 Identification of Type of Pump Used for SRPR....................................2-1

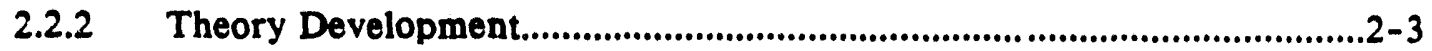

2.2.3 Solution Method and Head..................................................................

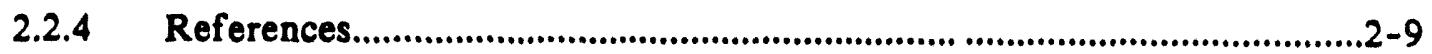

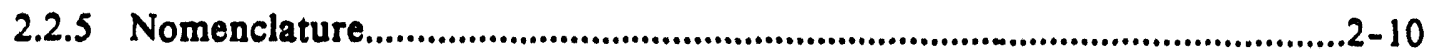

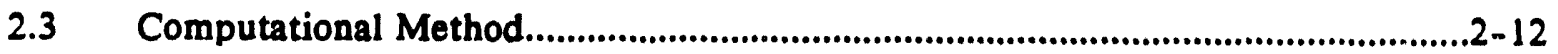

$2.4 \quad$ Numerical Results--Head and Torque...............................................................

2.5 Correlation between the Calculated Results and

Experimental Results for Cavitation Inception

of Savannah River Reactor Pump.................................................................2-13

Table 2.2.1-1

Representative Geometric Data of SRPR Pump.

$.2-18$

Table ?.4.1a-f

Head and Torque vs. Cavitation No. of SRPR Pump with Flow Rate as a Parameter for both AC Motor (1000rpm) and DC Motor (300rpm) Operations.

Figure 2.2.1-1

Typical Plan and Side Vies of a Radial Pump.

Figure 2.2.1-2

Inner and Outer Radius of SRP Pump. $.2-26$

Figure 2.2.1-3

Proof of Logarithmic Spiralness of SRP Pump. $2-27$

Figure 2.2.2-1

Physical Plane $z=x+i$. $.2-28$

Figure 2.2.2-2

Linear Cascade Plane $\omega=\phi+i \psi$ After Mapping of z-plane. $.2-28$

Figure 2.2.2-3

Mixed Boundary Value Plane $\zeta=\xi+i \eta$. . 2-29

Figure 2.4.1

Cavitation Characteristics of SRPR Pump Performance. $2-30$ (AC Motor Operation, 1000rpm) 
Figure 2.4.2

Figure 2.5.1

Figure 2.5.2
Cavitation Characteristics of SRPR Pump Performance (DC Motor Operation, 300rpm)



Appendix 2B Derivation of the Cavity Closure Condition.....................................................

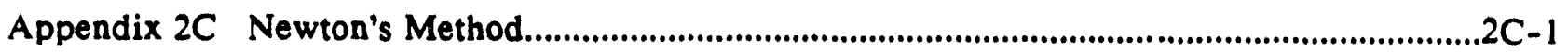

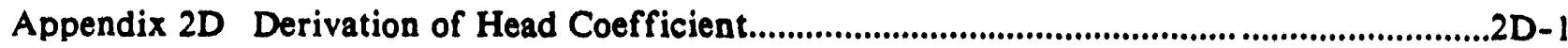

3. Air/Water Two-Phase Flow Performance of SRPR Pump Under AC Motor Operation (Task 3, Task 9 and Task 15)............................................................................

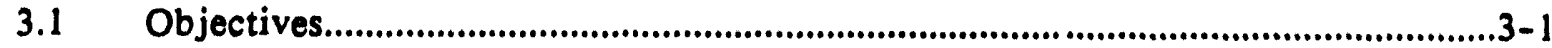

3.2 Theoretical Basis---Without Diffuser (Task 3)...................................................

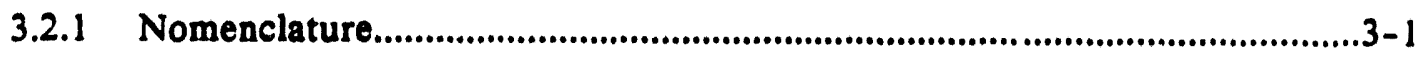

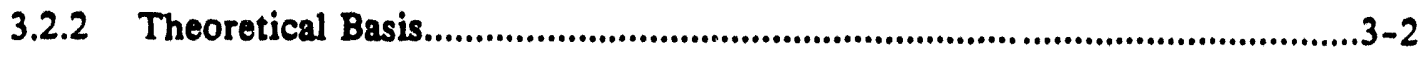

3.2.3 Solution Method...........................................................................................

3.2.4 Two-Phase Flow Head of Pump (Task 3)...............................................3-5

3.2.5 Theoretical Basis---Homologous Torque (Task 9).....................................3-9

3.3 Numerical Calculations---Without Diffuser (Task 3)........................................3-11

3.3.1 Parametric Range....................................................................................

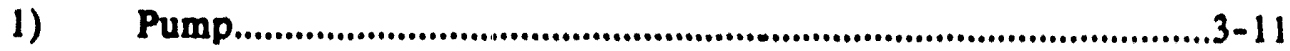

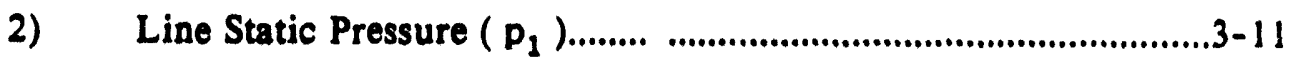

3) Flow Rate ( $Q$ ) and Pump Rotational Speed ( N ).......................



3.3.2 Results---Homologous Head (Task 3)...................................................3-12

3.3.3 Results-.--Homologous Torque (Task 9)...............................................3-13

3.4 Theoretical Basis---With Diffuser (Task 15).......................................................13 
3.5 Numerical Calculations---With Diffuser (Task 15),.........................................

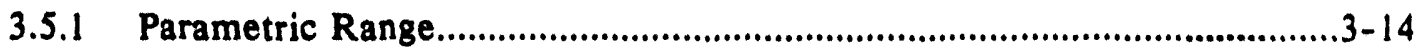

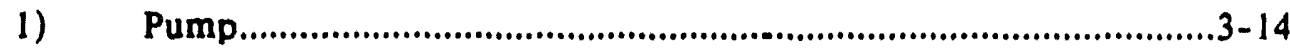

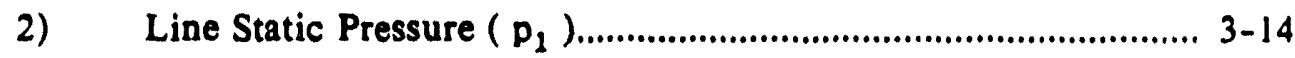

3) Flow Rate ( Q ) and Pump Rotational Speed ( N ).....................3-14

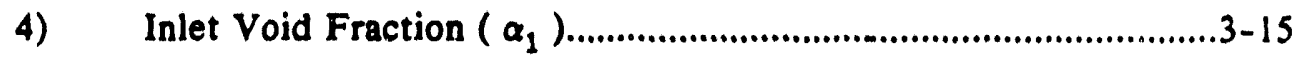

3.5.2 Result3---Detailed Flow Parameters (Task 15).......................................

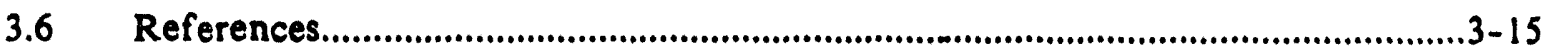

Figure 3.3.1-1 Bingham Pump head Characteristics at $1000 \mathrm{rpm}$ driven by AC Motor.

Figure 3.3.1-2 Bingham Pump Head Characteristics at $300 \mathrm{rpm}$ driven by DC Motor................................................................................

Figure 3.3.2-1a to l'd Head Degradation of SRPR Pump for Air/Water Flow

for Various Flow Parameters $\left(\nu / \alpha_{N}\right)$ at

Line Pressure of 3, 2, 1, and $0.05 \mathrm{~atm}$,

respectively (Without Diffuser)...

$3-19$ to $3-22$

Table 3.3.2-1a to Id Numerical Data used in Figures 3.3.2-la to Id (Without Diffuser)

Table 3.3.2-2a to 2d Void Fraction at Exit of Pump for the Same Cases as in Figures 3.3.2-1a to 1d (Without Diffuser). $3-27$ to $3-30$

Table 3.3.2-3a to 3d Static Pressure at Exit of Pump for the Same Cases as in Figure 3.3.2-la to Id (Without Diffuser). 3-34

Table 3.3.2-4a to $4 d$ Normalized Relative Velocities of Water at Exit of Pump for the Same Cases as in Figures 3.3.2-1a to Id (Without Diffuser)

Table 3.3.2-5a to 5d Normalized Relative Velocities of Air at Exit of Pump for the Same Cases as in Figures 3.3.2-1a to 1d (Without Diffuser) 
Table 3.3.2-6a to $6 \mathrm{~d}$ Homologous Torque Corresponding to Figures 3.3.2-1a to Id (Without Diffuser).

Table 3.5.2-1a to 1d Static Increase Pressure, Liquid Velocity, Gas Velocity, and Voia Fraction at Discharge Flange for $p_{1}=3,2,1$ and $0.5 \mathrm{~atm}$ (With Diffuser).

4 Air/Water Two-Phase Flow Performance of SRPR Pump Under DC Motor Operation (Task 7, Task 9 and Task 16).

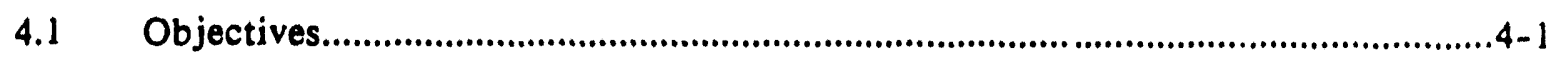

4.2 Theoretical Basis--- Without Diffuser.................................................................4-2

4.3 Numerical Calculations---Without Diffuser.....................................................4-3

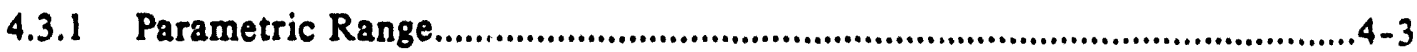

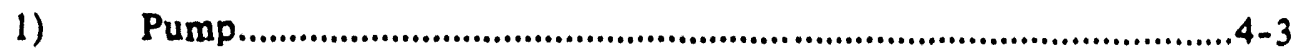



3) Flow Rate ( $Q$ ) and Pump Rotational Speed ( N )......................4-4

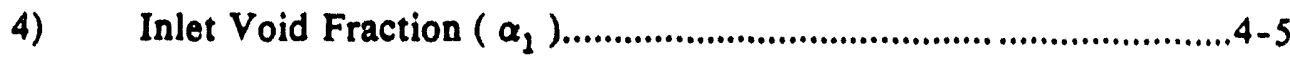

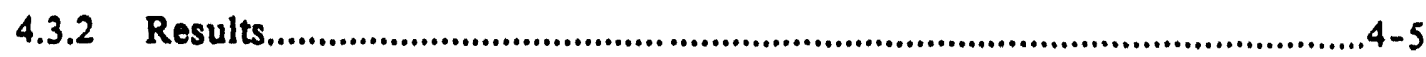

4.4 Theoretical Basis---With Diffuser...................................................................4-6

4.5 Numerical Calculations--- With Diffuser..........................................................4-6

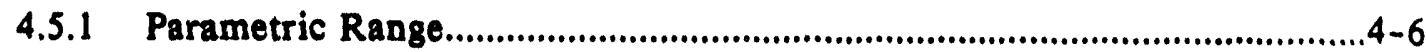

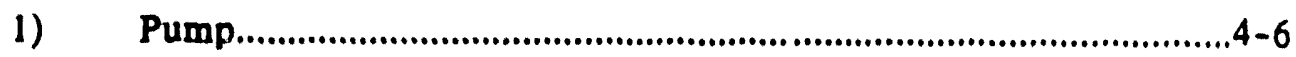

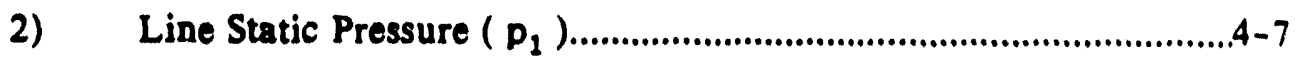

3) Flow Rate ( $Q$ ) and Pump Rotational Speed ( N ),.......................4-7

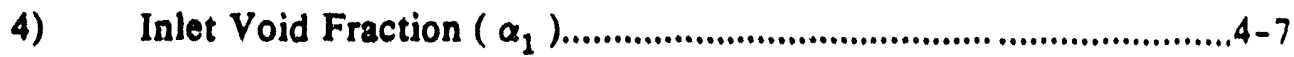

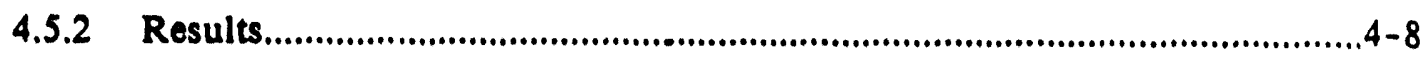

Figure 4.3.1-1 Bingham Pump head Characteristics at $1000 \mathrm{rpm}$ driven by $\mathrm{AC}$ Motor 
Figure 4.3.1-2

Figure 4.3.1-3
Bingham Pump Head Characteristics at $300 \mathrm{rpm}$ driven by DC Motor. $4-10$

Comparison of Pump Performance between AC and DC Motor Operations $4-11$

Figure 4.3.2-1a to Id Head Degradation of SRPR Pump for Air/Water Flow for Various Flow Parameters $\left(\nu / \alpha_{N}\right)$ at Line Pressure of 3, 2, 1, and 0.05 atm, respectively (Without Diffuser). $4-12$ to $4-15$

Table 4.3.2-1a to 1d Numerical Data used in Figures 4.3.2-1a to Id. $4-16$ to $4-19$ (Without Diffuser)

Table 4.3.2-2a to 2d Void Fraction at Exit of Pump for the Same Cases as in Figures 4.3.2-1a to 1d (Without Diffuser). $4-20$ to $4-23$

Table 4.3.2-3a to 3d Static Pressure at Exit of Pump for the Same Cases as in Figure 4.3.2-la to Id (Without Diffuser). $4-24$ to $4-27$

Table 4.3.2-4a to 4d Normalized Relative Velocities of Water at Exit of Pump for the Same Cases as in Figures 4.3.2-1a to Id. (Without Diffuser)

Table 4.3.2-5a to 5d Normalized Relative Velocities of A ir at Exit of Pump for the Same Cases as in Figures 3.3.2-1a to Id. (Without Diffuser)

Table 4.3.2-6a to $6 \mathrm{~d}$ Homologous Torque Corresponding to Figures 4.3.2-1a to Id (Without Diffuser). $4-36$ to $4-39$

Table 4.5.2-1a to $1 \mathrm{~d}$ Static Pressure Increase, Liquid Velocity, Gas Velocity, and Void Fraction at Discharge Flange for $p_{1}=3,2,1$ and 0.5 atm (With Diffuser). $.4-40$ to $4-55$

5. SRPRP Head and Torque Characteristics Under Steam/Water and Steam/Air/Water Conditions for Both AC and DC Motor Operations Without Diffuser Section (Tasks 4, 8, 11 and 12)

5.1 Theoretical Basis

5.2 Numerical Results for Stea/Water Two-Phase Flow Cases Unsder the AC 


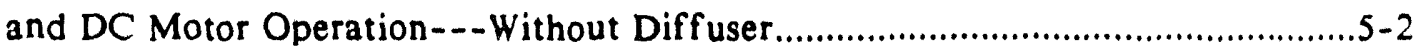

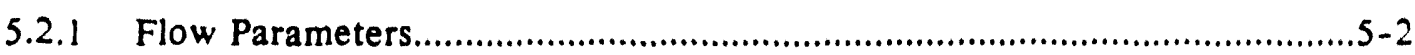

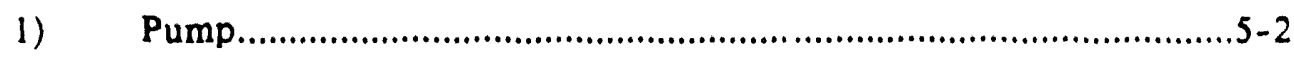

2) Line Static Pressure $\left(p_{1}\right)$......................................................5.-4

3) Flow Rate ( $Q$ ) and Pump Rotational Speed ( N )....................5-4

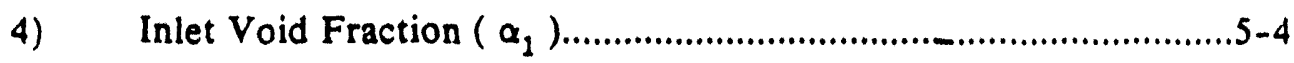

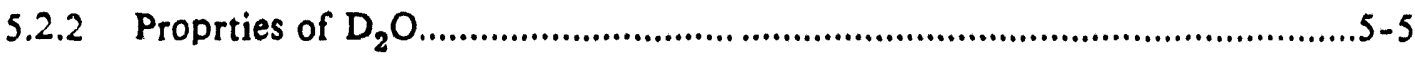

5.2.3 Numerical Results for AC and DC Motor Opeations................................5-5

Figure 5.2.1 to 5.2.4 Head Degradation of SRPR Pump Under AC Motor Operation for Steam/Water Flow for Various Flow Parameters $\left(\nu / \alpha_{N}\right)$ at Line Pressure of 3, 2, 1, and 0.05 atm, respectively $5-8$ to $5-11$

Figure 5.2.5 to 5.2.8 Head Degradation of SRPR Pump Under DC Motor Operation for Steam/Water Flow for Various Flow Parameters $\left(\nu / \alpha_{N}\right)$ at Line Pressure of 3, 2, 1, and $0.05 \mathrm{~atm}$, respectively. $.5-32$ to $5-35$

Table 5.2.1

Steam Table for $\mathrm{D}_{2} \mathrm{O}$ $.5-7$

Table 5.2.2 to 5-2-5 Numerical Data used in Figures 5.2.1 to 5.2.4...........................5-12 to 5-31

Table 5.2.6 to 5-2-9 Numerical Data used in Figures 5.2.5 to 5.2.8.............................5-36 to 5-55 


\section{Introduction}

The possible head degradation of the SRPR pumps may be attributable to two independent phenomena, one due to the inception of cavitation and the other due to the two-phase flow phenomena. The head degradation due to the appearance of cavitation on the pump blade is hardly likely in the conventional pressurized water reactor (PWR) since the coolant circulating line is highly pressurized so that the cavitation is difficult to occur even at LOCA (loss of coolant accident) conditions. (Note that the line pressure at PWR is as high as 2500 psi., see the paper of Furuya(1985)).) On the other hand, the suction pressure of SRPR pump is order-of-magnitude smaller than that of PWR so that the cavitation phenomena may prevail, should LOCA occur, depending on the extent of LOCA condition. In this study, therefore, both cavitation phenomena and two-phase flow phenomena were investigated for the SRPR pump by using various analytical tools and the numerical results are presented herein. In the following the contents of each section will be briefly described.

\section{Section 2:}

The objective of Task 2, Task 6, Task 13 and Task 14 is therefore to determine the performance of the SRPR pump operating under the cavitating conditions. The tasks involved are therefore i) to identify the type of pump used for the SRPR since the type of the analytical tool varies, depending on the geometry of the pump. Then, ii) a theory is developed to analyze the performance of the SRPR pump operating under the cavitating condition with the intention to conduct a numerical analysis based on the theory developed herein.

It has been found however that numerical difficulties existed with the analytical method so that a cemputational methed was employed to determine the performance of the cavitating pump including both the head and torque. The contents included herein correspond to Task 2a (a theory development), Task $2 b$ (the head calculation for the AC motor operation), Task 6 (that for the DC motor operation), Task 13 (the torque calculation for the AC motor operation) and Task 14 (that for the DC motor operation).

\section{Section 3:}

Under the hypothetical loss of coolant accident (LOCA) conditions, there is a possibility that the water level of the coolant tank becomes so low that air may be ingested into the water line. The coolant pump then experiences the air/water flow going through the blades. As has been demonstrated by experiments conducted by Babcock \& Wilcox (1977) and Creare (1982) as well as the

$$
1-1
$$


theory of Furuya (1985), the head of the pump operating under the air/water two-phase flow condition severely degrades as the inlet void fraction reaches about $20 \%$ or higher (although this threshold point for the degradation varies depending on the type of pump and operational conditions). It is crucial from the reactor safety viewpoints to determine the extent of the pump head degradation either theoretically or experimentally. However, this type of experiments is too costly and time consuming.

The objective of Task 3, Task 9 and Task 15 is therefore to theoretically determine the head and torque degradation of the SRPR pump operating under the air/water two-phase flow conditions for various physical, hydrodynamic and mechanical conditions. The parameters which represent such conditions include the inlet void fraction, static pressure at upstream, flow rate and pump rotational speed. During the normal cooling operation, the SRPR pump is driven by the AC motor at $1,000 \mathrm{rpm}$ as the rated speed, while under the emergency condition, it is operated by the DC motor at $300 \mathrm{rpm}$ as its rated speed. The head vs. flow rate curves between these two oparations are quite different.

Two types of pump now configurations were used for the present work. One is the pump configuration normally used for the single flow design and analysis where the pump blade flow passage is used and the diffuser effect is ignored or treated empirically in terms of the head loss and torque change (Tasks 3 and 9). The other is the pump configuration with diffuser section added. (Task 15)

In Tasks 3 and 9 the air/water flnw pump performance under the $A C$ motor operation were calculated without diffuser over a range of the line static pressure of $0.05,1,2$ and $3 \mathrm{~atm}$, inlet void fraction of 0.00 to 0.98 and normalized flow parameter of 0.4 to 1.4. The normalized head and torque are presented in the form of figures as well as of tables. Furthermore, the detailed data obtained as the results of the pump calculations are provided, including the void fraction, static pressure and water/air relative flow velocities at exit of the pump blade.

In Task 15 similar calculations were made with the pump diffuser incorporated over a range of the line static pressure of $0.5,1,2$ and $3 \mathrm{~atm}$, inlet void fraction of 0.00 to 0.98 and normalized flow parameter of 0.02 to 1.4. The static pressure increase, liquid velocity, gas velocity and void fraction at the discharge flange section were obtained and presented in tabular form.

\section{Section 4:}


In Task 7 and Task 9, the air/water flow pump performance including the head and torque degradation under the DC motor operation without the diffuser section was calculated over a range of the line static pressure of $0.05,1,2$ and $3 \mathrm{~atm}$, inlet void fraction of 0.00 to 0.98 and normalized flow parameter of 0.4 to 1.4. In Task 16, similar calculations were made with the pump diffuser section incorporated for the same parameters as those used above except for the static pressure of 0.5 atm instead of 0.05 and the homologous parameter of 0.2 added. The normalized head is presented both in the form of figures as well as of tables for Task 7 and only tables are provided for Task 16. Furthermore, for Task 7, the detailed data obtained as the results of the pump calculations are provided, including the void fraction, static pressure and water/air relative flow velocities at exit of the pump blade.

\section{Section 5:}

The objective of Tasks $4,8,11$ and 12 is to theoretically determine the head and torque degradation of the SRPR pump operating with both $A C$ and DC motors under the steam/water two-phase flow conditions for various physical, hydrodynamic and mechanical conditions. The parameters which represent such conditions include the inlet void fraction, static pressure at upstream, flow rate and pump rotational speed. During the normal cooling operation, the SRPR pump is driven by the AC motor at 1,000 rpm as the rated speed, while under the emergency condition, it is operated by the DC motor at $300 \mathrm{rpm}$ as its rated speed.

The mathematical formula for analyzing the steam/water two-phase flow pump performance was developed some time ago and has been used many times for the purpose of comparing the calculated results with existing experimental results. The correlations obtained to date were found out to be 8ood. The major difference between the previous calculations and the present case lies in the fact that the static line pressure of the previous cases was in a range of 2000 psi since the coolant pumps are for the pressurized water reactor, whereas that of the SRPRP case is $0.05 \mathrm{~atm}(0.7 \mathrm{psi})$ to $3 \mathrm{atms}$ (44 psi). This large difference in the static pressure made the numerical computations extremely unstable since the enthalpy changes so abruptly as a function of the pressure for the latter case.

The results of computations showed fair stability for the $A C$ motor operation while those for the DC motor operation showed total instability for lower pressure cases.

The causes for such instability were investigated, but no definite conclusions have been made to date, requiring further investigation for stabilizing the numerical calculations for the low pressure twophase flow cases. 
2. Cavitating Flow Performance of SRPR (Savannah River Plant Reactor) Pumps (Tusks 2, 6, 13 and 14)

\subsection{Objectives}

The possible head degradation of the SRPR pumps may be attributable to two independent phenomena, one due to the inception of cavitation and the other due to the two-phase flow phenomena. The head degradation due to the appearance of cavitation on the pump blade is hardly likely in the conventional pressurized water reactor (PWR) since the coolant circulating line is highly pressurized so that the cavitation is difficult to occur even at LOCA (loss of coolant accident) conditions. (Note that the line pressure at PWR is as high as 2500 psi., see the paper of Furuya(1985)).) On the other hand, the suction pressure of SRPR pump is order-of-magnitude smaller than that of PWR so that the cavitation phenomena may prevail, should LOCA occur, depending on the extent of LOCA condition.

The objective of Tasks 2, 6, 13 and 14 is therefore to determine the extent of head degradation of SRPR pump when the pump starts cavitating. More specifically, when the suction pressure comes down to the atmospheric pressure, is there still any positive head generated by the pump ? In order to answer these questions, a proper anaiytical tool needs to be developed or selected.

The first task is therefore to identify the type of pump used for the SRPR since the type of the analytical tool varies, depending on the geometry of the pump. Once the type of the SRPR pump is identified, an anlytical or numerical methode is developed or selected to determine the cavitation characteristics of the pump.

\subsection{Develupment of Analytical Model}

\subsubsection{Identification of Type of Pump Used for SRPR}

Based on the available hardware data, the radii of various blade points along the hub (called the "inner radii" and indicated by "RI") and those along the hub (called the "outer radii" and indicated by "RO", were measured as a function of the angle. The results are shown in Table 2.2.1-1 and Figure 2.2 1 - : (see also Figure 2.2.1-1 which shows a schematic diagram of the plan and side views of the pums 
blade).

If this blade profile shape is made as a well-known logarithmic spiral, the angular coordinate $\theta$ and radius $r$ should satisfy the following relationship

$$
\theta=\cot \beta \cdot \ln r / r_{1}
$$

where the definitions of $\theta, \beta, r$ and $r_{1}$ are shown in Figure 2.2.1-2.

Using $r_{1}$ and $r_{2}$ (i.e., the innermost radius and outermost radius) in Eqni. $(2.2 .1-1)$ for both $R I$ and $R O$, $\beta$ can be calculated as follows;

\begin{tabular}{|c|c|c|}
\hline & RI & RO \\
\hline$r_{1}$ (inch) & 7.05 & 9.78 \\
$r_{2}$ (inch) & 20.5 & 21.0 \\
\hline$\beta$ (degree) & $15.071^{\circ}$ & $10.790^{\circ}$ \\
\hline
\end{tabular}

With this $\beta$ value used, the angle, $\theta$, was recalculated by using Eqn. $(2.2 .1-1)$. If this blade profile is made truly in a logarithmic spiral shape, the calculated angle should match with the measured one exactly. The last two columns of Table $2.2 .1-1$ show such results, indicating that the blade profile shape along the hub of the SRPR pump is made exactly in a logarithmic spiral form with $\beta=15.0710$. Figure 2.2.1-3 shows the plottings of the angle as a function of station number. the straight line actually consists of the measured and calculated values based on the radius along the hub (RI) which totally match with each other. On the other hand, the calculated value based on the radius along the shroud (RO) forms a curved line which somewhat deviates from the straight line. This fact indicates that the blade shape along the hub is made exactly in a logarithmic spiral shape while that along the shroud slightly deviates from it. However, it is considered to be a fair assumption that the SRPR pump is essentially designed in a logarithmic spiral profile shape so that very little error will be generated by using this assumption for the cavity flow analysis. 


\subsubsection{Theory Development}

A radial pump of logarithmic spiral blades can be conveniently mapped into a linear cascade by a well-known mapping function. A conformal mapping method combined with the mixed boundary value problem solution method will be used for the development of a supercavitating pump flow theory.

\section{Boundary Conditions}

The Bernoulli equation in the rotating frame gives the following relationship

$$
\rho\left(w^{2}-u^{2}\right) / 2+p=p_{t} \text { (constant) }
$$

where $u$ is the circumferential velocity, $w$ the relative velocity, $\rho$ the liquid density, $p$ the static pressure and $p_{t}$ the total pressure which is constant along the flow. The static pressure $p$ inside or on the boundaries of the cavity is assumed to be that of the vapor pressure of the liquid $p_{v}$, therefore,

$$
w_{u}=u_{2} \sqrt{\sigma+\left(r / r_{2}\right)^{2}}
$$

where $\sigma$ is defined as the cavitation number

$$
\begin{aligned}
\sigma & =\left(p_{t}-p_{v}\right) /\left(\frac{1}{2} \rho u_{2}^{2}\right) \\
& =\left[\rho\left(w_{1}^{2}-u_{1}^{2}\right) / 2+p_{1}-p_{v}\right] /\left(\frac{1}{2} \rho u_{2}^{2}\right)
\end{aligned}
$$

Under the assumption that the cavity boundary is close to the blade,

$$
w_{c}=w_{*}
$$

where $w_{1}$ is the relative flow velocity tangent to the blade surface. The flow tangency condition is then applied to the blade surface:

$$
w_{n}=0
$$


It should be noted that the relative flow velocities in the rotating machinery, $w_{n}$ and $w_{\text {, consist of }}$ four separate components, i.e., the blade through flow due to the flow rate $Q$, the prerotation $\Gamma_{1}$, the rotation of blade $(\Omega)$ and the perturbation components $\left(v_{n}{ }^{\prime}, v_{a}^{\prime}\right)$ due to the existence of blade and cavity,

$$
\begin{aligned}
& w_{n}=(Q / 2 \pi r) \cos \beta-\left(r \dot{n}-\Gamma_{i} / 2 \pi r\right) \sin \beta+v_{n}^{\prime} \\
& w_{s}=(Q / 2 \pi r) \sin \beta+\left(r \Omega-\Gamma_{i} / 2 \pi r\right) \cos \beta+v_{i}
\end{aligned}
$$

$v_{n}$ 'and $v_{a}^{\prime}$ in these equations are the irrotational components so that all the tools available for the potential theory are conveniently employed. In terms of these potential flow components, the boundary conditions on the blade and cavity are now written:

i) on the blade:

$$
v_{n}^{\prime}=-(Q / 2 \pi r) \cos \beta+\left(r n-\Gamma_{i} / 2 \pi r\right) \sin \beta
$$

ii) on the cavity boundary:

$$
\begin{array}{r}
v_{1}^{\prime}=u_{2} \sqrt{\sigma+\left(r / r_{2}\right)^{2}}-(Q / 2 \pi r) \sin \beta \\
-\left(r \Omega-\Gamma_{1} / 2 \pi r\right) \cos \beta
\end{array}
$$

\section{Conformal Mappings}

The blades of logarithmic spiral profile shape in the physical plane are first mapped to a linear cascade plane $\omega$, which is then mapped to the upper half of a mixed-boundary value problem plane ५. The first mapping is achieved by

$$
\omega=N e^{-i r} \ln \left(z / r_{1}\right)
$$

where $\omega=\phi+i \psi$, which maps $N$ logarithmic spiral blades shown in Figure 2.2.2-1 to a linear cascade of stagger angle $\gamma$ having a spacing of $2 \pi$ (see Figure 2.2.2-2) and $\omega$ is defined in Figure 2-2-2-3. The normal and tangential velocity components, $v_{n}{ }^{\prime}$ and $v_{i} ;$ in the $z$-plane are related to those in the $\omega$-plane through the following equations 


$$
\begin{aligned}
& v=-(r / N) v_{n}^{\prime} \\
& u=(r / N) v_{i}^{\prime}
\end{aligned}
$$

where the complex potential $F$ in the $z$ - and $\omega$-plane has been utilized as follows:

$$
\begin{aligned}
d F / d \omega & =u-i v \\
d F / d z e^{i \theta} & =v_{r}-i v_{\theta} \\
& =\left(v_{n}-i v_{n}\right) e^{i \beta} \\
d F / d \omega & =(d F / d z)(d z / d \omega) .
\end{aligned}
$$

The second mapping is performed through the following mapping function (see the paper of Sutherland and Cohen (1958))

$$
\begin{aligned}
\omega & =e^{i \gamma} \ln \left[\left(1-5 / 5_{1}\right) /\left(1-5 / 5_{2}\right)\right] \\
& +e^{-i \gamma} \ln \left[\left(1-5 / 5_{1}^{*}\right) /\left(1-5 / 5_{2}^{*}\right)\right]
\end{aligned}
$$

where

$$
\begin{aligned}
& \zeta_{1}=e^{i(\pi / 2-w)}, \zeta_{2}=a e^{i(\pi / 2+w)} \\
& \zeta_{1}^{*} \cdot \zeta_{2}^{*} \text { are the complex conjugates of } \zeta_{1} \text { and } \zeta_{2}
\end{aligned}
$$

and the mapping parameters $a$ and $w$ are determined through the scaling between the two planes as follows;

$$
\begin{aligned}
\tan w & =[(a-1) /(a+1)] \tan \gamma \\
c / 2 \pi & =(\cos \gamma \cdot \ln a+2 w \sin \gamma) / \pi .
\end{aligned}
$$


$\zeta_{1}$ and $\zeta_{2}$ in the $\zeta$-plane correspond to the points at the upstream and downstream infinities in the $\omega$-plane where the end point of cavity, $\omega_{T}$ is now mapped to the infinity point in the $\zeta$-plane (see Figure 2.2.2-3). The coordinate relationship between the $\zeta$ - and $\omega$-planes is given

$$
\begin{aligned}
& \phi(\xi)=\cos \gamma \cdot\left[\ln \left(1+\xi^{2}-2 \xi \cdot \sin w\right)-\right. \\
& \left.\ln \left(1+(\xi / a)^{2}+2(\xi / a) \sin w\right)\right]+ \\
& 2 \sin \gamma \cdot \tan ^{-1}\left[\left((a-1) \xi \cos w+\xi^{2} \sin 2 w\right)\right] \\
& \left.\left(a-(a-1) \xi \sin w+\xi^{2} \cos 2 w\right)\right] \text {. }
\end{aligned}
$$

\section{Mixed-Type Boundary Value Problem}

The velocity potential $W(\omega)=W(\zeta)$ is defined

$$
W(\omega)=W(\zeta)=u-i v
$$

Through an analytical continuation of W $(\zeta)$ into the lower half of the 5 -plane

$$
W\left(\zeta^{*}\right)=-W(\zeta)=-u-i v \text {, }
$$

the boundary conditions are now written either by the real or imaginary part of $W($ ), i.e., u or $v$, in the following form;

$$
\begin{aligned}
& w^{+}+w^{-}=-2 i v \\
& w^{+}-w^{-}=2 u .
\end{aligned}
$$

where the superscript " + " or " $"$ indicates the limiting value of W approaching from the positive or negative (or upper or lower) side of the $\xi$-axis. Therefore, on the real axis of the 5 -plane, i.e., $\xi$-axis.

$$
\begin{aligned}
& -\infty<\xi<-b ; W^{+}-W^{-}=2 u \\
& -b<\xi<0 ; W^{+}+W^{-}=-2 i v
\end{aligned}
$$




$$
0<\xi<\infty ; W+-W^{-}=2 u
$$

This mixed type boundary value problem may be solved by applying the Plemelj's theory (see the text

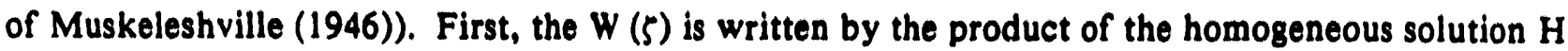
$(\zeta)$ and the general solution $G(\zeta)$

$$
W(5)=H(\zeta) G(5)
$$

By assuming the singularity at the leading edge and no singularity at the trailing edge of the blade, the following form of the homogeneous solution is selected

$$
H(5)=\sqrt{(5+b) / 5}
$$

where the branch cut on the real axis $\xi$ from $-b$ to 0 has been introduced. It is noted however that although no singularity was specifically selected for the cavity end point at this stage, the mapping function automatically provides a quarter root singularity at the cavity end similar to that of the leading edge of the blade. The homogeneous function has the following characteristics on the real axis of 5 -plane

$$
\begin{aligned}
& -\infty<\xi<-b \quad ; H^{+}(\xi)=\sqrt{(\xi+b) / \xi}=H^{-}(\xi) \\
& -b<\xi<0 \quad ; H^{+}(\xi)=\sqrt{-(\xi+b) / \xi / i}=-H^{-}(\xi) \\
& 0<\xi<\infty \quad ; H^{+}(\xi)=\sqrt{(\xi+b) / \xi}=H^{-}(\xi) .
\end{aligned}
$$

In terms of $G(\varsigma)$,

$$
\begin{aligned}
G^{+}-G^{-} & =(W / H)^{+}-(W / H)^{-} \\
& =\left(W^{+}-W^{-}\right) / H^{+}-2 u / \sqrt{(\xi+b) / \xi,}-\infty<\xi<-b \\
& =\left(W^{+}+W^{-}\right) / H^{+}=2 v / \sqrt{-(\xi+b) / \xi,}-b<\xi<0
\end{aligned}
$$




$$
=\left(W^{+}-W^{-}\right) / H^{+}=2 u / \sqrt{(\xi+b) / \xi}, \quad 0<\xi<\infty .
$$

The application of the Plemelj's theorem yields to

$$
\begin{aligned}
& W(\zeta)=H(\zeta) G(\zeta) \\
& =\sqrt{(\zeta+b) / \zeta\left[(1 / 2 \pi i) \int_{-\infty}^{\infty}\left(G^{+}-G^{-}\right) d \xi^{\prime} /\left(\xi^{\prime}-\zeta\right)+P(\zeta)\right]} \\
& =\sqrt{(\zeta+b) / \zeta\left[(1 / 2 \pi i) \int_{-\infty}^{b} 2 u / \sqrt{\left(\xi^{\prime}+b\right) / \xi^{\prime}} d \xi^{\prime} /\left(\xi^{\prime}-\zeta\right)+\right.} \\
& \quad(1 / 2 \pi i) \int_{-b}^{0} 2 v / \sqrt{-\left(\xi^{\prime}+b\right) / \xi^{\prime}} d \xi^{\prime} /\left(\xi^{\prime}-\zeta\right)+ \\
& (1 / 2 \pi i) \int_{0}^{\infty} 2 u / \sqrt{\left(\xi^{\prime}+b\right) / \xi^{\prime}} d \xi^{\prime} /\left(\xi^{\prime}-\zeta\right) \\
& +P(\zeta)]
\end{aligned}
$$

where $P(\zeta)=\Sigma C_{n} \zeta^{n}(n=-\infty$ to $+\infty)$.

However, $P(\zeta)$ cannot have any stronger singularity at $\zeta=0$, only the form $P(\zeta)$ can take is

$$
P(\zeta)=A \zeta+B
$$

where $A$ and $B$ are real constants to be determined. Further, it should be noted that the mapping function between the $\omega$ - and 5 -planes provides the following relationship as has been mentioned earlier

$$
5 \approx 1 /\left(\omega-e^{2}\right)^{1 / 2} ; 5 \rightarrow \infty
$$

where $e^{\prime}$ is the cavity length in the $w$-plane.

\subsubsection{Solution Method and Head}


In the previous section, the perturbed complex velocities, $W=u-i v$, have been obtained with the boundary conditions assumed to be known. In this expression there exist three unknown parameters, i.e., b, A and B. b represents the coordinate for the cavity length so that, if the cavity length in the physical plane $z$ is given, $b$ can be calculated through the mapping functions. These three unknown parameters are uniquely determined by three independent boundary conditions; two of them are provided from the perturbed flow velocities at the upstream infinity and the third one is provided by the cavity closure condition:

$$
\begin{aligned}
& W\left(\zeta_{1}\right)=0+i \cdot 0 \quad \text { (two equations) } \\
& \operatorname{Re}\left[W\left(\zeta_{2}\right) e^{-i \gamma}\right]=0 \quad \text { (one equation) }
\end{aligned}
$$

See Appendix $2 \mathrm{~A}$ for the detailed expressions for Eqns. (2.2.3-1) and $(2.2 .3-2)$ and Appendix $2 \mathrm{~B}$ for the derivation of the cavity closure condition.

The three equations obtained above are linear in terms of $A$ and $B$, but nonlinear in terms of $b$.

The objective of the present study is to determine the maximum cavity length when the static pressure at inlet drops to the vapor pressure and then to obtain the corresponding head. Therefore, the problem is posed in the following manner. By assuming $p_{1}=p_{v}$, the cavitation number is determined based on the given flow rate $Q$ and pump rotation speed $n$. The three equations obtained above are solved for b, A and B by using the Newton's iterative procedure (see Appendix $2 C$ for the more explicit explanation for the Newton's method). The head is then obtained at this limiting condition by balancing the angular momentum

$$
H=\operatorname{Im}\left[W\left(\zeta_{2}\right) e^{-i \gamma}\right] N \Omega / 8
$$

Appendix 2D provides the detailed derivation of this formula.

\subsubsection{References}

Furuya, O., 1975, "Exact Supercavitating Cascade Theory", J. of Fluids Eng., ASME, vol. 97, no.4, 419-429.

$$
2-9
$$


Furuya, O., 1980, "Nonlinear Theory for Partially Cavitating Cascade Flows", Proc. 10th Symp. of IAHR, Tokyo, 221-241.

Furuya, O., 1985, "An Analytical Model for Prediction of Two-Phase (Noncondensable) Flow Pump Performance", J. of Fluids Eng., ASME, vol. 109, 139-147.

Muskhelishvili, N. I., 1946, Singular Integral Equations, P. Noordhoff Ltd., Gronigen, Holland.

Sutherland, C. D. and Cohen, H., 1958, "Finite Cavity Cascade Flow", Proc. 3rd. Nat'l Congress of Applied Mechanics, 837-845.

\subsubsection{Nomenclature}

2. one of two mapping parameters between the w-plane and 5-plane

b: the 8 -coordinate representing the blade trailing edge

c,c': the blade chord length in the physical and the linea cascade plane, respectively

F: complex potential (dF/dw $=u-i v)$

8: the gravitational acceleration

G: the general solution to the Riemann-Hilbert boundary problem

H: the hydrodynamic head; also used as the homoseneous solution to the present Riemann-Hilbert boundary value problem

L C: length of cavity in the physical (z-) and w-plane, respectively

p: the static pressure

$p_{1}$ : the total pressure (see Equation 2.2.2-1)

Pri the vapor pressure

Q: Now rate

r. the redial coordinate in the physical (2-)plane

Re,Im: the rnd and imaginary parts of the complex quantities, respectively

u,v: the purturbed velocities in the physical (z-)plane

$u^{\prime}, v^{\prime}:$ the perturbed velocities in the mapped (w-)plane

$u_{1}, u_{2}:$ the circumforential velocities at inlet and exit of the blade, respectively

$v_{p}, v_{j}$ the radial and circumferential velocities in the physical (z-)plane, respectively

$w_{1}, w_{2}$ : the relative flow velocities at inlet and exit of the blade in the rotating frame

W: the velocity potential in the 5 -plane $(-u-i v)$

z. the physical plane $(-x+i y)$ 
B: the blade angle (see Figure 2.2.2-1 and Equation 2.2.1-1)

$\gamma$. the complementary blade angle $(=\pi / 2-\beta)$

$\Gamma_{1}$ : the strength of the pre-swirl at inlet of the pump

5: $\quad$ the mapped plane $(-\xi+i \eta)$

$\zeta_{1}, \zeta_{2}:$ the 5 coordinates corresponding to the upstream and downstream infinity points, respectively

б. cavitation number (see Equation 2.2.2-3)

w. one of two mapping parameters for the mapping between the $\omega$-plane and 5 -plane

w: mapped plane $(-\phi+i \phi)$

ก: $\quad$ pump rotational speed

\section{Superscripts and Subscripts:}

1,2: the quantities at inlet and exit of the pump, respectively

c: the quantity related to the cavity

$\mathrm{n}$ : the quantity normal to the blade surface

s: the quantity parallel to the blade surface 


\subsection{Computational Method}

As an alternative to the potential theory described above, the computational method may be considered to determine the performance of the cavity flow of the SRPR pump. In this method the discrete vortex $y$ is distributed over the entire flow boundaries including the pump blade and cavity boundaries. The induced velocities $u$ and $v$ will then be expressed

$$
(u-i v)(z)=Q / 2 \pi z+\sum_{n=1}^{N} 1 / 2 \pi \int i \gamma_{n}(s) d s /\left(z-s_{n}(s)\right)
$$

where $\zeta_{n}$ is the coordinate on the boundaries and $\gamma_{n}(8)$ is the strength of the vortex. The first term of Eqn. (2.3.1-1) is the contribution of the source of the flow similar to those in Eqns. $(2.2 .2-6)$ and $(2.2 .2-7)$. The boundary conditions on the solid boundary and the cavity remain the same as those used for the potential flow theory in Section 2.2

\subsection{Numerical Results.--Head and Torque Calculations}

In order to determine the performance of the SRPR pump operating under the cavitating conditions, either of the methods described above may be used. However, due to the aumerical difficulties expected with the potential theory, thecomputntionnl mathod was used herein to obtain the results.

The torque of the cavitating pump has been calculated through the conventional input-power-tohydraulic-energy relationship,

$$
T=(08 Q \mathrm{H}) /(\eta \omega)
$$

where the pump cfrieibacy of the single-phase flow provided in the report of E.I. du Pont, Savannah River Laboratory, No. DPST-88-777 has been used. The power lose eaused by the form drug due to the cavitation was not include since the lose of head is 80 mpld and the power increase due to such term will not play any essential role in the system hydraulic analyals.

Figure 2.4.1 and Figure 2.4.2 show the head in terms of feet vs. cavitation aumber with the flow rate as a parameter for the AC motor of $1000 \mathrm{rpm}$ and the DC motor of $300 \mathrm{rpm}$, respectively. The 
conversion from the cavitation number $\sigma$ to the line static pressure $p$ is readily made by use of the following relatior:

$$
\sigma=\left(p_{1}-p_{v}\right) /\left(t \rho u_{2}^{2}\right)
$$

where

$p_{1}=p+\rho v_{p 2} / 2$ (total head at inlet of the pump) $p=$ line static pressure (pounds $/ \mathrm{ft}^{2}$ )

$v_{r}=Q \times 0.00223 /\left(4 \pi_{1} b_{1}\right)$

$Q=$ flow rate ( $8 \mathrm{pm})$

$r_{1}=$ the inner radius of the blacle ( $16.25 / 2 / 12$ in feet)

$b_{1}=$ the flow channel width at inlet ( $5.7 / 12$ in feet)

$\rho$ - the density of the heavy water ( slug $/ \mathrm{ft}^{3}$ )

$p_{v}=$ the vapor pressure of the heavy water (which is the function of temperature, pounds $\left./ \mathrm{ft}^{2}\right)$

$u_{2}=2 \pi \Omega r_{2}$

$\Omega$ - pump rotational speed in rps (rounds per second, l.e., for the AC motor $\Omega=1000 / 60$ and for the DC motor $n=300 / 60$ )

$r_{2}$ - the outer radius of the blade ( $39.5 / 2 / 12$ in feet)

Tables 2.4.1a through 2.4.1f provide the detail data for the head in terms of feet and the torque in terms of Ibf-ft vs. cavitation number for both the AC motor and DC motor cases with the flow rate as a parameter.

\subsection{Correlation between the Calculated Results and Experimental Results for Cavitation Inception of Savannah River Reactor Pump}

The cavitation number $\sigma$ is defined as

$$
\sigma-\left(p_{1}-p_{v}\right) /\left(1 / 2 \rho u_{2}^{2}\right)
$$

where

$$
\begin{aligned}
p_{1} & =p+\rho v_{r}^{2} / 2 \\
p & =\text { line static pressure } \\
v_{r} & =\text { flow velocity at inlet of blade } \\
& =Q \times 0.00223 /\left(4 \pi r_{1} b_{1}\right) \\
Q & =\text { flow rate in spm } \\
p_{v} & =\text { vapor pressure }
\end{aligned}
$$




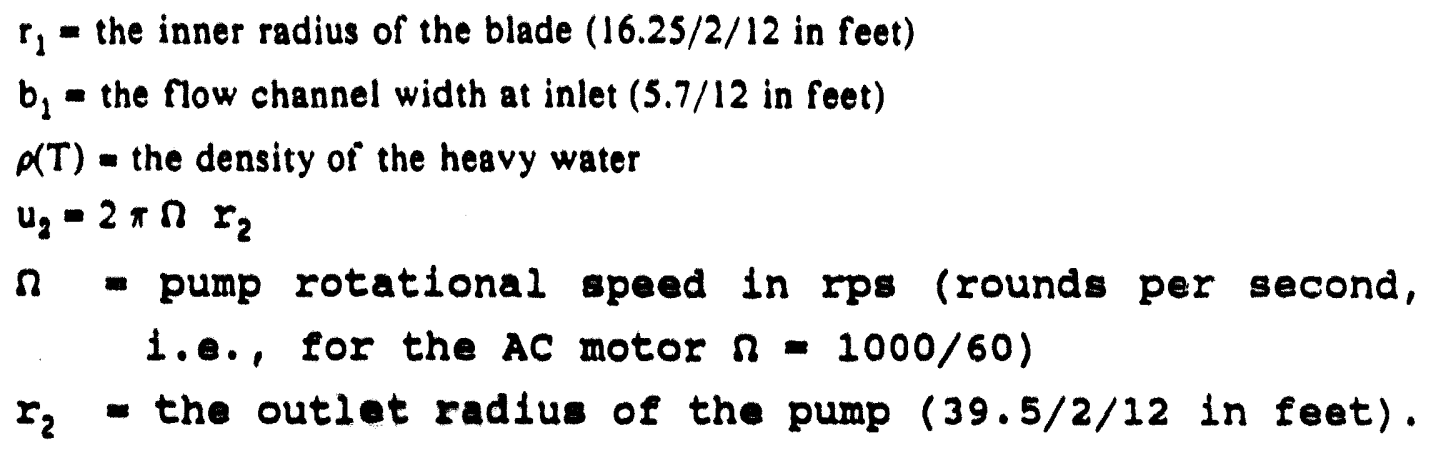

On the other hand, NPSH used in EGEG Report NO. EGG-EAST-8148 (1988) was deflned

$$
\text { NPSH }=\left(p-p_{v}\right) / \gamma(T)+v_{p e}{ }^{2} / 28
$$

where

$$
\begin{aligned}
& \text { NPSH = net positive suction head, feet } \\
& \begin{array}{r}
v_{p t}=\text { average cross sectional velocity at pump suction, } \\
\text { feet } / \text { sec. }\left(=Q \times 0.00223 / \pi(23.25 / 12 / 2)^{2}\right)
\end{array} \\
& \gamma(T)=\text { specific weight of the liquid }\left(=p(T)_{B}\right) .
\end{aligned}
$$

From Equations $(2.5-1)$ and $(2.5-2)$, the relationship between the cavitation number and NPSH is given,

$$
\mathrm{NPSH}=\sigma u_{2}^{2} / 2 g+\left(v_{p 0}^{2}-v_{r}^{2}\right) / 2 g
$$

By using Equation (2.5-3), the calculated cavitation numbers $\sigma$ for inception shown in the OF Tech report mentioned above are converted into NPSH and shown in Table 2.5-1 below.

The experimental data for the NPSH where the head starts degrading are read from the Figure F2 at page 235 of the EG\&G report mentioned above and are shown in Table 2.5-2. 
Table 2.5-1 NPSH vs Cavitation Inception Number $\sigma$ as a Function of Flow Rate

\begin{tabular}{|c|c|c|}
\hline$Q(g p m)$ & $\sigma$ & $\begin{array}{c}\text { NPSH in feet } \\
(\text { calc.) }\end{array}$ \\
\hline 9,391 & 0.008 & 4.19 \\
15,652 & 0.0130 & 7.25 \\
18,782 & 0.0150 & 8.66 \\
25,043 & 0.0125 & 8.65 \\
28,173 & 0.1150 & 58.21 \\
34,434 & 0.3800 & 186.16 \\
\hline
\end{tabular}

These two results are plotted in Figure 2.5-1, indicating that the first four points of the (NPSH) cale values smoothly blend into those of the experimental data. It seems to be quite reasonable to utilize both the calculated results for the low flow rate range and the experimental data for the high flow rate range for determining the threshold points for head degradation. More specifically, the first four data points of calculation and all four experimental data points were used to determine the best fitted polynomial equation of third order:

$$
\mathrm{NPSH}_{1}=a_{0}+a_{1} Q+a_{2} Q^{2}+a_{3} Q^{3}+\Delta \operatorname{NPSH}_{1}(T), \Omega=1000 \mathrm{rpm} \quad(2.5-4)
$$

where

$$
\begin{aligned}
\text { NPSH }_{1}= & \begin{array}{l}
\text { net positive suction head at which the } \\
\text { cavitation occurs or the head degradation } \\
\text { starts occurring (feet) }
\end{array} \\
Q & =\text { flow rate in Kgpm } \\
a_{0} & =-35.3366 \\
a_{1} & =7.57104 \\
a_{2} & =-0.441945 \\
a_{3} & =0.00868303
\end{aligned}
$$

$\Delta \mathrm{NPSH}_{i}(\mathrm{~T})$ a correction term due to the fluid temperature (feet) 


$$
\begin{aligned}
& =b_{0}+b_{1}(T-95)+b_{2}(T-95)^{2}+b_{3}(T-95)^{3} \\
b_{0} & =0 . \\
b_{1} & =-0.0908805 \\
b_{2} & =-0.000745422 \\
b_{3} & =-1.337 E-06 \\
T & =\text { temperature in } \cdot C .
\end{aligned}
$$

$\begin{aligned} \text { Table } 2.5-2 & \text { NPSH-expt. as a Function of Flow Rate } \\ \text { at } T & =95^{\circ} \mathrm{C}\end{aligned}$

\begin{tabular}{|c|c|}
\hline Q (gpm) & $\begin{array}{c}\text { NPSH in feet } \\
\text { (expt.) }\end{array}$ \\
\hline 22,000 & 11.5 \\
25,000 & 14.5 \\
28,000 & 18.0 \\
30,000 & 30.0 \\
\hline
\end{tabular}

The correction term for the fluid temperature for the $\mathrm{NPSH}_{1}$ value was obtained from Figure F3 of the EG\&G report which shows the effect of the fluid temperature on the cavitation inception or the NPSH value at which the head starts degrading. The procedure for determining the $\triangle N P S H_{i}(T)$ was that first the difference of NPSH values from those at $95^{\circ} \mathrm{C}$ were plotted as a function of the fluid temperature for all the flow rate data (see Figure 2.5-2) and the best fitted polynomial curve was obtained.

Equation 2.5-4 now can be used as the threshold point where the head starts degrading. The slope of degradation may be determined by following the slope shown in Figure F of EG\&G report for the flow rate of $22 \mathrm{Kgpm}$ or higher and that of dropping almost vertically to a degree as long as numerical stability is guaranteed for the lower flow rate.

It should be noted that when NPSH value hits 0 as the flow rate $Q$ becomes smail (defining this $Q$ as $Q_{c}$ ), NPSH $=0$ should be used for the flow rate equal to $Q_{c}$ or smaller. 
For the rotational speed different from the AC motor rated speed, i.e., $\Omega=1000 \mathrm{rpm}$, the following step should be applied for determining (NPSH),

1) Step 1:

Find the geometrically similar flow rate $Q_{\mathbf{g}}$ from the following relationship:

$$
Q_{.}=(1000 / \Omega) Q
$$

where $\Omega$ is the rotational speed at which the pump is operated and $Q$ is the flow rate.

2) Step 2:

Use Equation (2.5-4) to find the corresponding NPSH, at which the pump would cavitate if it were at $\Omega=1000$.

3) Step 3:


and $Q$. used.

4) Step 4:

Recalculate NPSH ( $=\mathrm{NPSH}_{\mathrm{i}}$ ) by using Equation (2.5-4) again with now real $\Omega$ and $Q$ for $\mathrm{NPSH}_{1}$ to be obtained. 
Table 2.2.1-1

Representativa Geometric Data of SRPR Pump

\begin{tabular}{|c|c|c|c|c|c|c|}
\hline $\begin{array}{l}\text { Eation } \\
\text { No. }\end{array}$ & $\begin{array}{l}\text { Angle } \\
\text { from } \\
\text { left } \\
\text { (degree) }\end{array}$ & $\begin{array}{l}\text { Angle } \\
\text { from } \\
\text { right } \\
\text { (degree) }\end{array}$ & $\begin{array}{c}\text { Outer } \\
\text { Radius } \\
\text { (inch) } \\
\text { RO }\end{array}$ & $\begin{array}{c}\text { Inner } \\
\text { Radius } \\
\text { (inch) } \\
\text { RI }\end{array}$ & $\begin{array}{c}\text { Logarit } \\
\text { Spiral } \\
\text { based } \\
\text { Ro }\end{array}$ & $\begin{array}{l}\text { mic } \\
\text { itting } \\
\text { on } \\
\quad \text { RI }\end{array}$ \\
\hline $\begin{array}{r}1.0 \\
1.5 \\
2.0 \\
2.5 \\
3.0 \\
3.5 \\
4.0 \\
4.5 \\
5.0 \\
5.5 \\
6.0 \\
7.0 \\
8.0 \\
9.0 \\
10.0 \\
11.0 \\
12.0 \\
13.0 \\
14.0 \\
15.0 \\
16.0 \\
16.5\end{array}$ & $\begin{array}{r}0.0 \\
7.5 \\
15.0 \\
22.5 \\
30.0 \\
37.5 \\
45.0 \\
52.5 \\
60.0 \\
67.5 \\
75.0 \\
90.0 \\
105.0 \\
120.0 \\
135.0 \\
150.0 \\
165.0 \\
180.0 \\
195.0 \\
210.0 \\
225.0 \\
232.5\end{array}$ & $\begin{array}{r}232.5 \\
225.0 \\
217.5 \\
210.0 \\
202.5 \\
195.0 \\
187.5 \\
180.0 \\
172.5 \\
165.0 \\
157.5 \\
142.5 \\
127.5 \\
112.5 \\
97.5 \\
82.5 \\
67.5 \\
52.5 \\
37.5 \\
22.5 \\
7.5 \\
0.0\end{array}$ & $\begin{array}{r}21.00 \\
20.25 \\
19.58 \\
18.90 \\
18.21 \\
17.61 \\
16.98 \\
16.40 \\
15.84 \\
15.30 \\
14.78 \\
13.83 \\
12.94 \\
12.18 \\
11.52 \\
10.98 \\
10.60 \\
10.30 \\
10.03 \\
9.87 \\
9.80 \\
9.78\end{array}$ & $\begin{array}{r}20.50 \\
19.80 \\
19.11 \\
18.47 \\
17.83 \\
17.23 \\
16.63 \\
16.10 \\
15.55 \\
15.00 \\
14.52 \\
13.57 \\
12.62 \\
11.76 \\
11.00 \\
10.28 \\
9.60 \\
8.95 \\
8.35 \\
7.78 \\
7.27 \\
7.05\end{array}$ & $\begin{array}{r}232.50 \\
221.44 \\
211.20 \\
200.44 \\
189.13 \\
178.94 \\
167.85 \\
157.28 \\
146.71 \\
136.15 \\
125.63 \\
105.42 \\
85.18 \\
66.77 \\
49.82 \\
35.21 \\
24.50 \\
15.76 \\
7.68 \\
2.79 \\
0.62 \\
0.00\end{array}$ & $\begin{array}{r}232.50 \\
224.93 \\
217.21 \\
209.79 \\
202.10 \\
194.65 \\
186.93 \\
179.87 \\
172.30 \\
164.46 \\
157.37 \\
142.64 \\
126.83 \\
111.45 \\
96.90 \\
82.16 \\
67.25\end{array}$ \\
\hline
\end{tabular}


Table 2.4.1a

Head vs. Cavitation No. of SRPR Pump with Flow Rate as a Parameter for both AC Motor (1,000rpm) and DC Motor (300rpm) Operations

\begin{tabular}{|c|c|c|c|c|}
\hline \multirow{2}{*}{$\begin{array}{l}\text { Cavitation } \\
\text { No. } \\
\text { (8igma) }\end{array}$} & \multicolumn{2}{|c|}{$\begin{array}{c}\text { AC Motor } \\
(Q=9,391 \mathrm{gpm})\end{array}$} & \multicolumn{2}{|c|}{$\begin{array}{c}\text { DC Motor } \\
(Q=2,8 I 7 \mathrm{gpm})\end{array}$} \\
\hline & $\begin{array}{l}\text { Head } \\
\text { (feet) }\end{array}$ & $\begin{array}{l}\text { Torque } \\
\text { (Ibf-ft) }\end{array}$ & $\begin{array}{l}\text { Head } \\
\text { (feet) }\end{array}$ & $\begin{array}{l}\text { Torque } \\
(1 b f-f t)\end{array}$ \\
\hline $\begin{array}{l}0.0600 \\
0.0500 \\
0.0400 \\
0.0300 \\
0.0200 \\
0.0180 \\
0.0160 \\
0.0140 \\
0.0120 \\
0.0100 \\
0.0080 \\
0.0070 \\
0.0069\end{array}$ & $\begin{array}{r}530 \\
530 \\
534 \\
543 \\
554 \\
557 \\
560 \\
563 \\
565 \\
566 \\
564 \\
96 \\
0\end{array}$ & $\begin{array}{r}11,583 \\
11,583 \\
11,670 \\
11,867 \\
12,107 \\
12,173 \\
12,238 \\
12,304 \\
12,348 \\
12,396 \\
12,326 \\
2,098 \\
0\end{array}$ & $\begin{array}{r}46.9 \\
46.9 \\
47.3 \\
48.1 \\
49.1 \\
49.4 \\
49.6 \\
49.9 \\
50.1 \\
50.2 \\
50.0 \\
7.9 \\
0.0\end{array}$ & $\begin{array}{r}1,025 \\
1,025 \\
1,034 \\
1,051 \\
1,073 \\
1,080 \\
1,084 \\
1,091 \\
1,095 \\
1,097 \\
1,097 \\
173 \\
0\end{array}$ \\
\hline
\end{tabular}


Table 2.4.1b

The Same as Table 2.4.1a except for Different Flow Rates

\begin{tabular}{|c|c|c|c|c|}
\hline \multirow[b]{2}{*}{$\begin{array}{c}\text { Cavitation } \\
\text { No. } \\
\text { (sigma) }\end{array}$} & \multicolumn{2}{|c|}{$\begin{array}{c}\text { AC Motor } \\
(Q=15,652 \mathrm{gpm})\end{array}$} & \multicolumn{2}{|c|}{$\begin{array}{c}\text { DC Motor } \\
(Q=4,696 \mathrm{gpm})\end{array}$} \\
\hline & $\begin{array}{l}\text { Head } \\
\text { (feet) }\end{array}$ & $\begin{array}{l}\text { Torque } \\
(\text { Ib } f-f t)\end{array}$ & $\begin{array}{l}\text { Head } \\
\text { (feet) }\end{array}$ & $\begin{array}{l}\text { Torque } \\
\text { (Ibf-ft) }\end{array}$ \\
\hline $\begin{array}{l}0.1000 \\
0.0850 \\
0.0645 \\
0.0600 \\
0.0580 \\
0.0500 \\
0.0400 \\
0.0300 \\
0.0200 \\
0.0165 \\
0.0130 \\
0.0120 \\
0.0119\end{array}$ & $\begin{array}{r}505 \\
510 \\
515 \\
522 \\
524 \\
526 \\
528 \\
530 \\
531 \\
532 \\
533 \\
136 \\
0\end{array}$ & $\begin{array}{r}14,079 \\
14,218 \\
14,358 \\
14,553 \\
14,609 \\
14,664 \\
14,720 \\
14,776 \\
14,804 \\
14,832 \\
14,860 \\
3,792 \\
0\end{array}$ & $\begin{array}{r}46.8 \\
47.3 \\
47.7 \\
48.4 \\
48.5 \\
48.7 \\
48.9 \\
49.1 \\
49.2 \\
49.3 \\
49.4 \\
13.7 \\
0.0\end{array}$ & $\begin{array}{r}1,305 \\
1,319 \\
1,330 \\
1,349 \\
1,352 \\
1,358 \\
1,363 \\
1,369 \\
1,372 \\
1,374 \\
1,377 \\
382 \\
0\end{array}$ \\
\hline
\end{tabular}


Table 2.4.1c

The Same as Table 2.4.1a except for Different Flow Rates

\begin{tabular}{|c|c|c|c|c|}
\hline \multirow[b]{2}{*}{$\begin{array}{c}\text { Cavitation } \\
\text { No. } \\
\text { (sigma) }\end{array}$} & \multicolumn{2}{|c|}{$\begin{array}{c}\text { AC Motor } \\
(Q=18,782 \mathrm{gpm})\end{array}$} & \multicolumn{2}{|c|}{$\begin{array}{c}\text { DC Motor } \\
(Q=5,635 \mathrm{gpm})\end{array}$} \\
\hline & $\begin{array}{l}\text { Head } \\
\text { (feet) }\end{array}$ & $\begin{array}{l}\text { Torque } \\
\text { (1bf-ft) }\end{array}$ & $\begin{array}{l}\text { Head } \\
\text { (feet) }\end{array}$ & $\begin{array}{l}\text { Torque } \\
\text { (1bt-ft) }\end{array}$ \\
\hline $\begin{array}{l}0.0700 \\
0.0600 \\
0.0500 \\
0.0450 \\
0.0400 \\
0.0300 \\
0.0200 \\
0.0180 \\
0.0160 \\
0.0150 \\
0.0145 \\
0.0144\end{array}$ & $\begin{array}{l}486 \\
486 \\
486 \\
489 \\
494 \\
508 \\
520 \\
524 \\
527 \\
529 \\
243 \\
0\end{array}$ & $\begin{array}{r}15,241 \\
15,241 \\
15,241 \\
15,335 \\
15,492 \\
15,931 \\
16,307 \\
16,433 \\
16,527 \\
16,589 \\
7,620 \\
0\end{array}$ & $\begin{array}{l}46.9 \\
46.9 \\
46.9 \\
47.1 \\
47.6 \\
48.8 \\
49.9 \\
50.3 \\
50.5 \\
50.7 \\
25.0 \\
0.0\end{array}$ & $\begin{array}{r}1,471 \\
1,471 \\
1,471 \\
1,477 \\
1,493 \\
1,530 \\
1,565 \\
1,577 \\
1,584 \\
1,590 \\
784 \\
0\end{array}$ \\
\hline
\end{tabular}


Table 2.4.1d

The Same as Table 2.4.1a except for Different Flow Rates

\begin{tabular}{|c|c|c|c|c|}
\hline \multirow{2}{*}{$\begin{array}{c}\text { Cavitation } \\
\text { No. } \\
\text { (sigma) }\end{array}$} & \multicolumn{2}{|c|}{$\begin{array}{c}\text { AC Motor } \\
(Q=25,043 \mathrm{gpm})\end{array}$} & \multicolumn{2}{|c|}{$\begin{array}{c}\text { DC Motor } \\
(Q=7,513 \mathrm{gpm})\end{array}$} \\
\hline & $\begin{array}{l}\text { Head } \\
\text { ( feet) }\end{array}$ & $\begin{array}{l}\text { Torque } \\
(1 b t-f t)\end{array}$ & $\begin{array}{l}\text { Head } \\
\text { ( Feet) }\end{array}$ & $\begin{array}{l}\text { Torque } \\
\text { (1bf-ft) }\end{array}$ \\
\hline $\begin{array}{l}0.0600 \\
0.0500 \\
0.0400 \\
0.0300 \\
0.0250 \\
0.0200 \\
0.0180 \\
0.0160 \\
0.0145 \\
0.0125 \\
0.0120 \\
0.0119\end{array}$ & $\begin{array}{r}429 \\
429 \\
430 \\
431 \\
432 \\
433 \\
435 \\
439 \\
441 \\
442 \\
299 \\
0\end{array}$ & $\begin{array}{r}17,349 \\
27,349 \\
17,389 \\
17,430 \\
17,470 \\
17,511 \\
17,591 \\
17,753 \\
17,834 \\
17,874 \\
12,092 \\
0\end{array}$ & $\begin{array}{r}47.2 \\
47.2 \\
47.3 \\
47.4 \\
47.5 \\
47.6 \\
47.8 \\
48.1 \\
48.3 \\
48.4 \\
35.5 \\
0.0\end{array}$ & $\begin{array}{r}1,909 \\
1,909 \\
1,913 \\
1,917 \\
1,921 \\
1,925 \\
1,933 \\
1,945 \\
1,953 \\
1,957 \\
1,436 \\
0\end{array}$ \\
\hline
\end{tabular}


Table 2.4.1e

The Same as Table 2.4.1a except for Different Flow Rates

\begin{tabular}{|c|c|c|c|c|}
\hline \multirow{2}{*}{$\begin{array}{c}\text { Cavitation } \\
\text { No. } \\
\text { (sigma) }\end{array}$} & \multicolumn{2}{|c|}{$\begin{array}{c}\text { AC Motor } \\
(Q=28,173 \mathrm{gpm})\end{array}$} & \multicolumn{2}{|c|}{$\begin{array}{c}\text { DC Motor } \\
(Q=8,452 \mathrm{gpm})\end{array}$} \\
\hline & $\begin{array}{l}\text { Head } \\
\text { (feet) }\end{array}$ & $\begin{array}{l}\text { Torque } \\
\text { (1bf-ft) }\end{array}$ & $\begin{array}{l}\text { Head } \\
\text { (feet) }\end{array}$ & $\begin{array}{l}\text { Torque } \\
\text { (Ibf- } t t)\end{array}$ \\
\hline $\begin{array}{l}0.3500 \\
0.3000 \\
0.2500 \\
0.2250 \\
0.2000 \\
0.1750 \\
0.1500 \\
0.1250 \\
0.1200 \\
0.1150 \\
0.1100 \\
0.1090\end{array}$ & $\begin{array}{l}387 \\
387 \\
388 \\
389 \\
391 \\
396 \\
401 \\
402 \\
404 \\
403 \\
242 \\
0\end{array}$ & $\begin{array}{r}18,144 \\
18,144 \\
18,191 \\
18,238 \\
18,332 \\
18,566 \\
18,801 \\
18,848 \\
18,942 \\
18,895 \\
11,346 \\
0\end{array}$ & $\begin{array}{r}47.5 \\
47.5 \\
47.6 \\
47.7 \\
47.9 \\
48.3 \\
48.8 \\
48.9 \\
49.1 \\
49.0 \\
34.5 \\
0.0\end{array}$ & $\begin{array}{r}2,227 \\
2,227 \\
2,232 \\
2,236 \\
2,245 \\
2,265 \\
2,288 \\
2,293 \\
2,302 \\
2,297 \\
1,618 \\
0\end{array}$ \\
\hline
\end{tabular}


Table 2.4.1f

The Same as Table 2.4.1a except for Different Flow Rates

\begin{tabular}{ccccc} 
& \multicolumn{2}{c}{$\begin{array}{c}\text { AC Motor } \\
(Q=34,434 \mathrm{gpm})\end{array}$} & $\begin{array}{c}\text { DC Motor } \\
(Q=10,330 \mathrm{pm})\end{array}$ \\
$\begin{array}{c}\text { Cavitation } \\
\text { No. }\end{array}$ & $\begin{array}{c}\text { Head } \\
\text { (feet) }\end{array}$ & $\begin{array}{c}\text { Torque } \\
\text { (1bin) }\end{array}$ & $\begin{array}{c}\text { Head } \\
\text { (fet) }\end{array}$ & Torque \\
\hline 0.7000 & 267 & 18,033 & 48.3 & 3,262 \\
0.6000 & 267 & 18,033 & 48.3 & 3,262 \\
0.5500 & 267 & 18,033 & 48.3 & 3,262 \\
0.4500 & 277 & 18,709 & 49.2 & 3,323 \\
0.4000 & 281 & 18,979 & 49.6 & 3,350 \\
0.3850 & 286 & 19,316 & 50.0 & 3,377 \\
0.3800 & 277 & 18,709 & 49.2 & 3,323 \\
0.3750 & 92 & 6,214 & 32.6 & 2,202 \\
0.3740 & 0 & 0 & 0.0 & 0
\end{tabular}



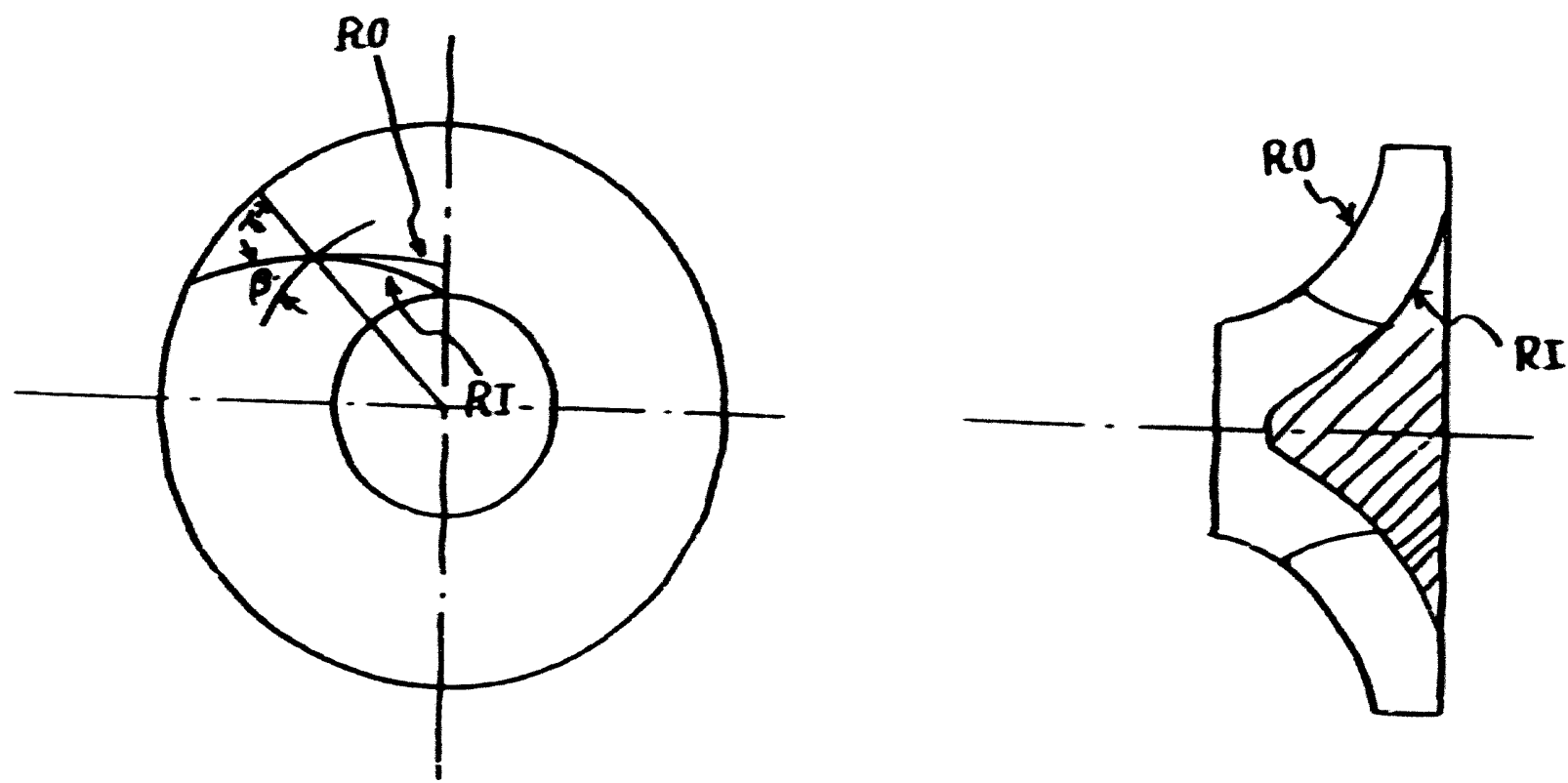

Figure 2.2.1-1

Typical Pan and Side Views of a Radial Pump 
Figure 2.2.1-2

\section{INNER AND OUTER RADIUS}

\section{OF SRP PUMP}

$\stackrel{2}{\alpha}$

$\frac{\sqrt{8}}{\frac{8}{3}}$

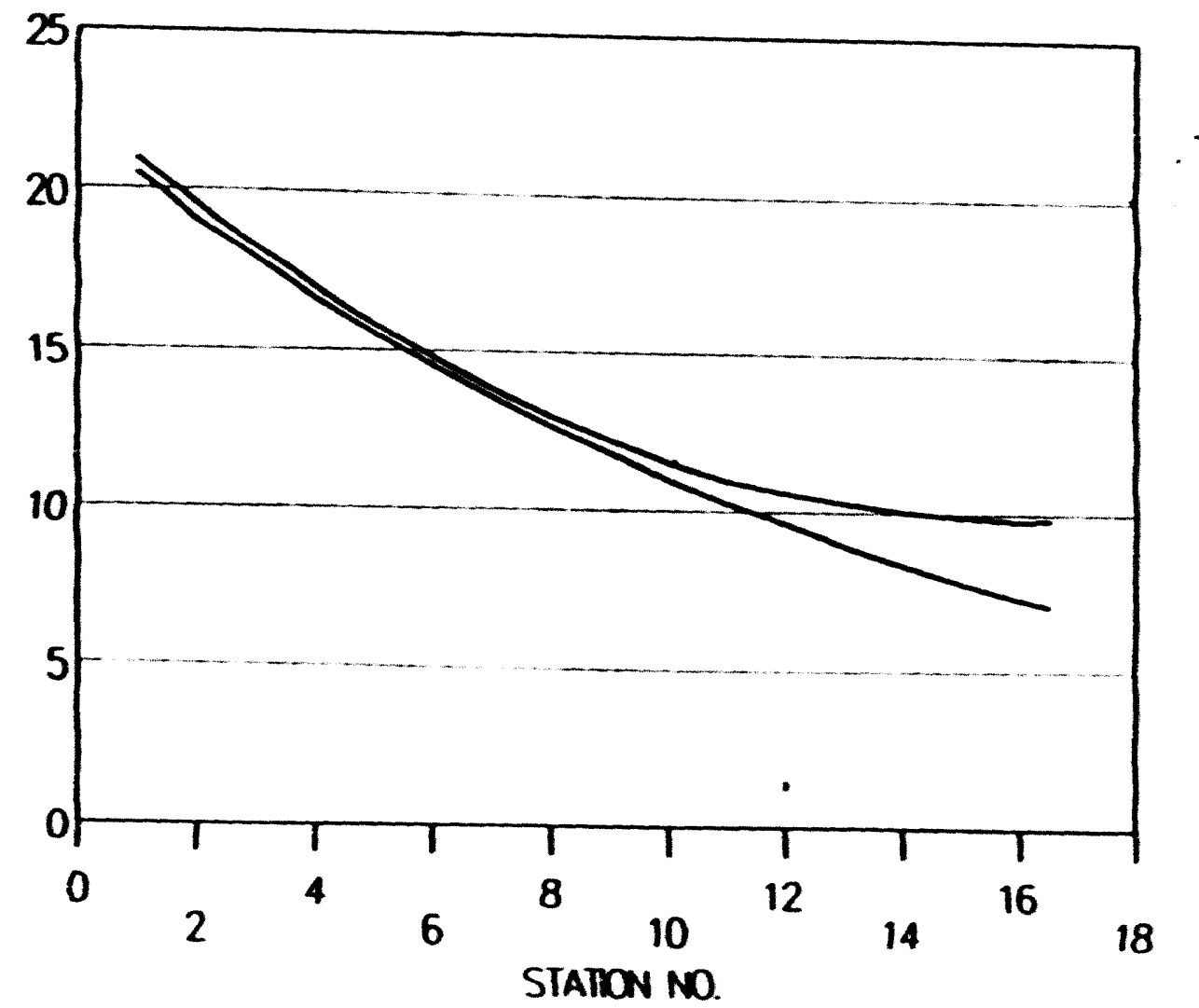


Figure 2.2.1-3

\section{PROOF OF LOGARITHMIC SPIRALNESS}

\section{OF SRP PUMP}

N

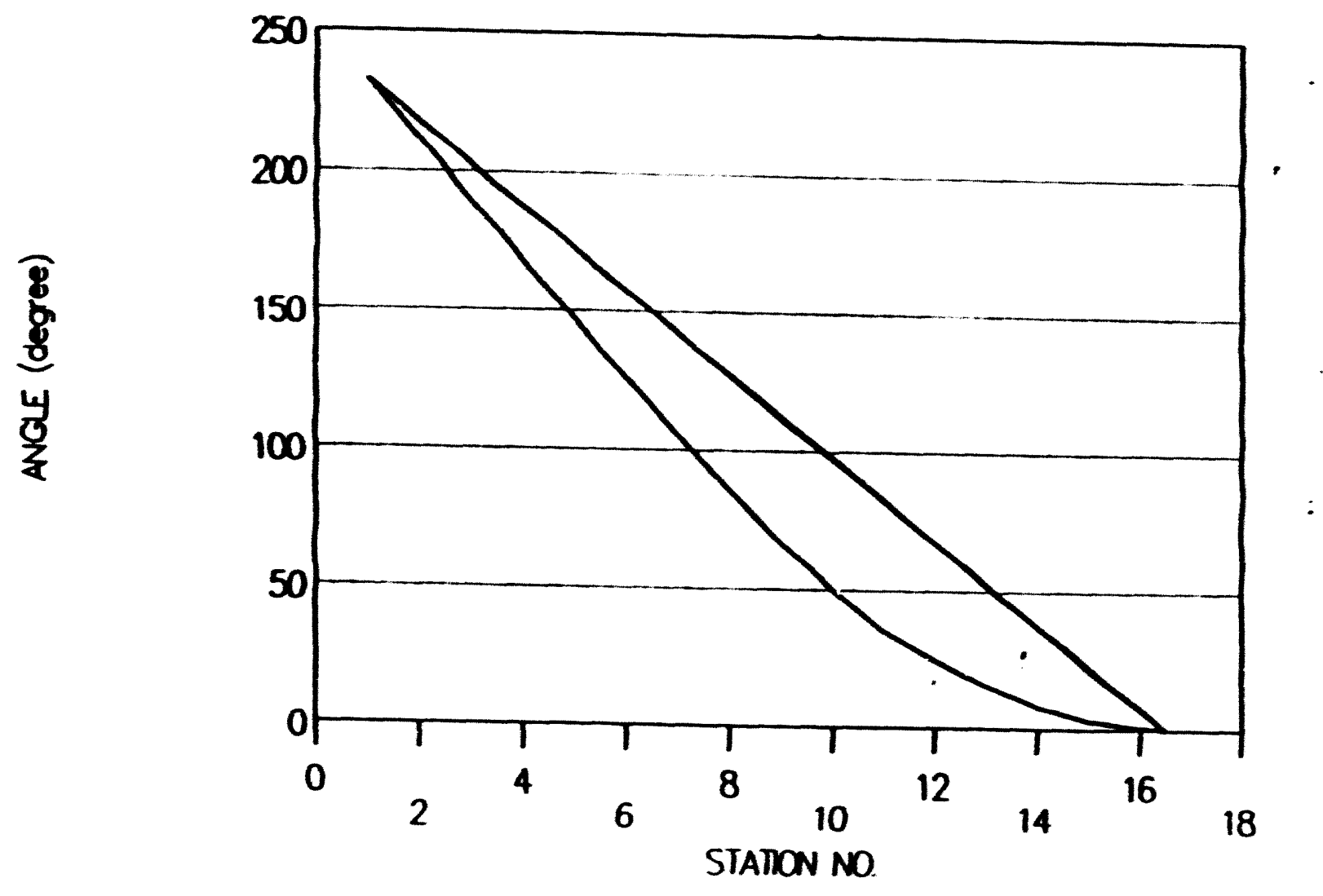






Figure 2.2.2-1

Physical Plane $z=x+i y$

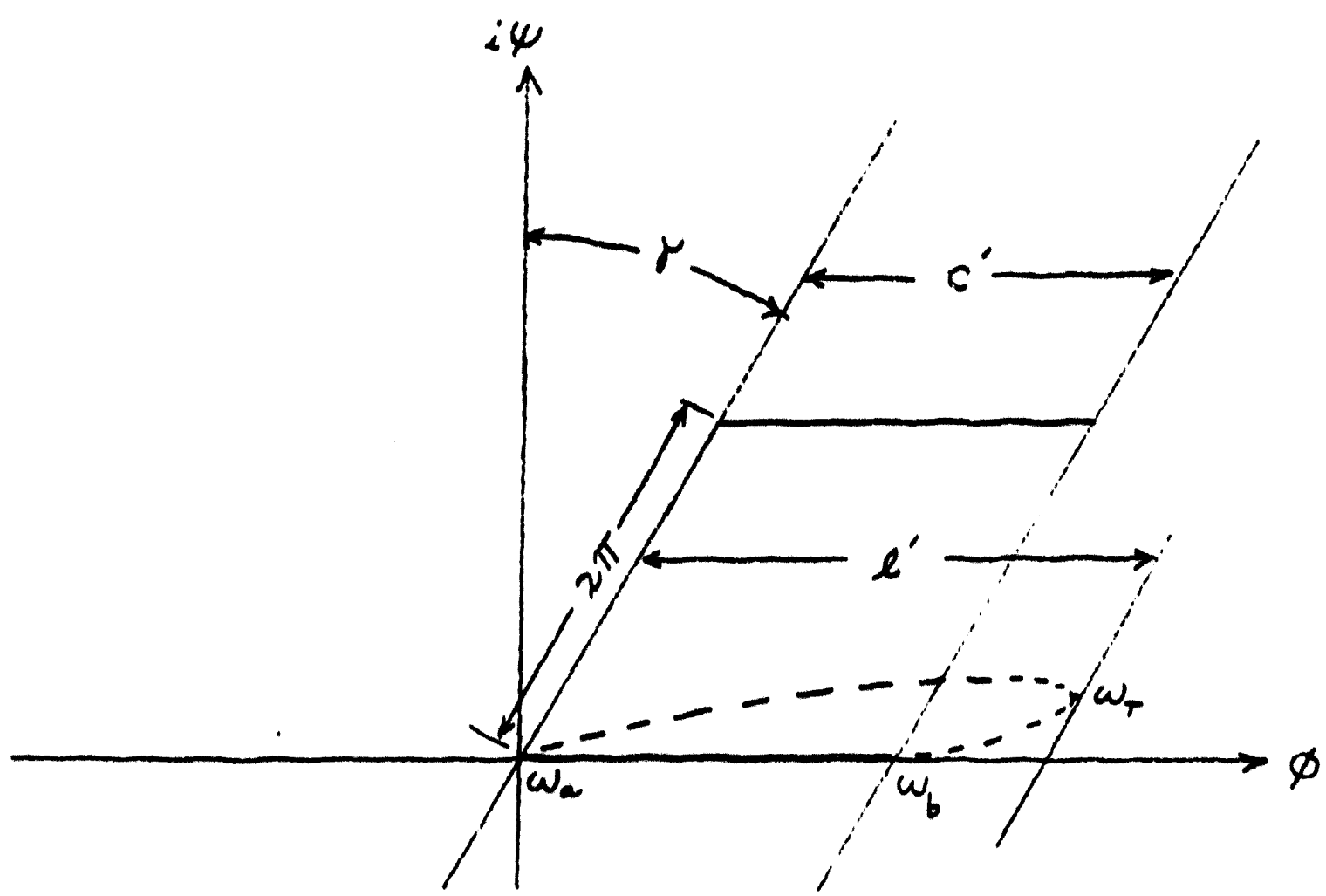

Figure 2.2.2-2

Linear Cascade Plane $w=\phi+i \phi$ After Mapping of z-plane 




Figure 2.2.2-3

Mixed Boundary Value Plane $\zeta=\xi+i n$ 


\section{Figure 2.4.1}

Cavitation Characteristics of SRPR Pump Performance

(AC Motor Operation, 1,000 rpm)

(See Section 2.4 for Conversion from

Cavitation No. $\sigma$ to Static Pressure p)

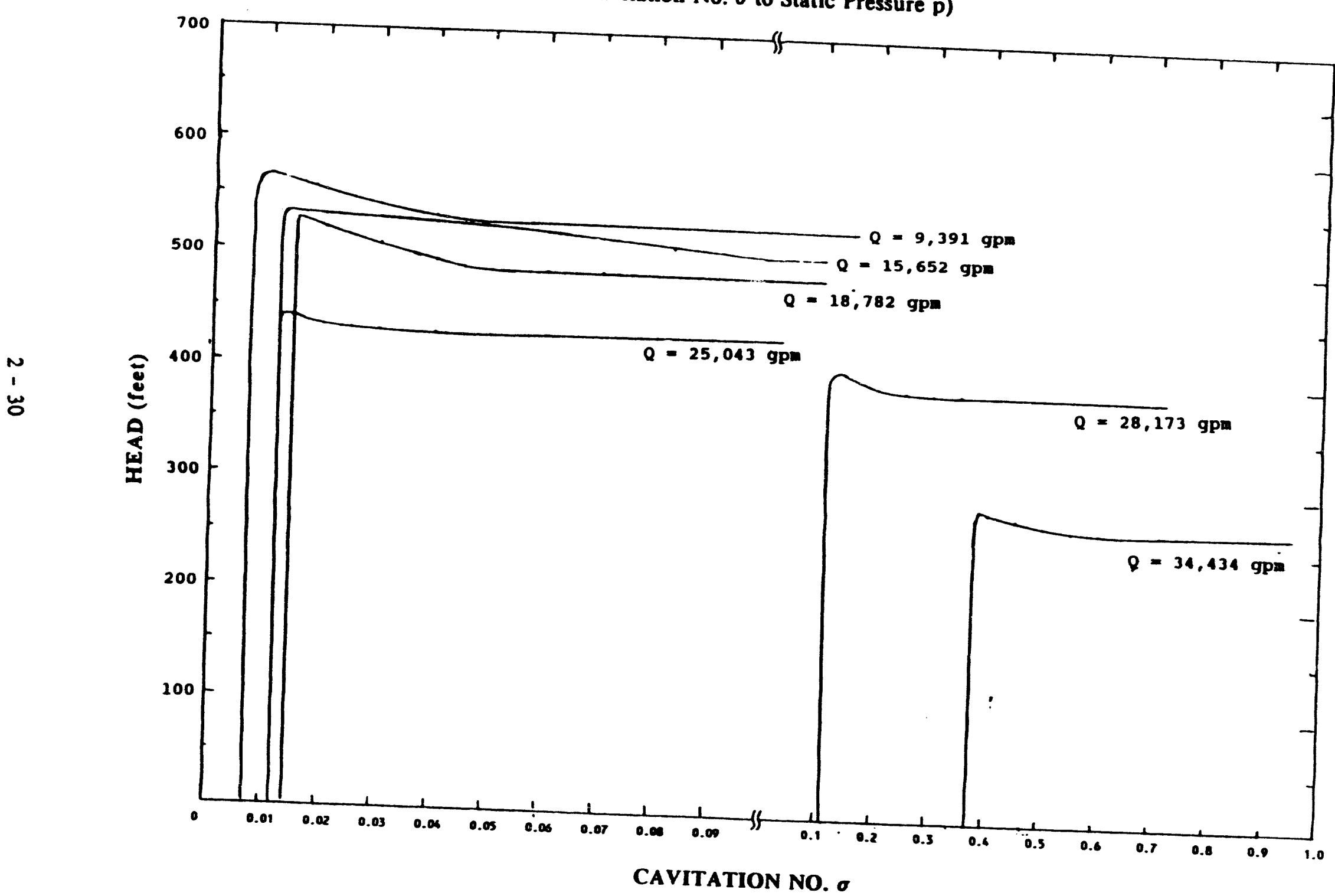


Figure 2.4.2

Cavitation Characteristics of SRPR Pump Performance (DC Motor Operation, $300 \mathrm{rpm}$ )

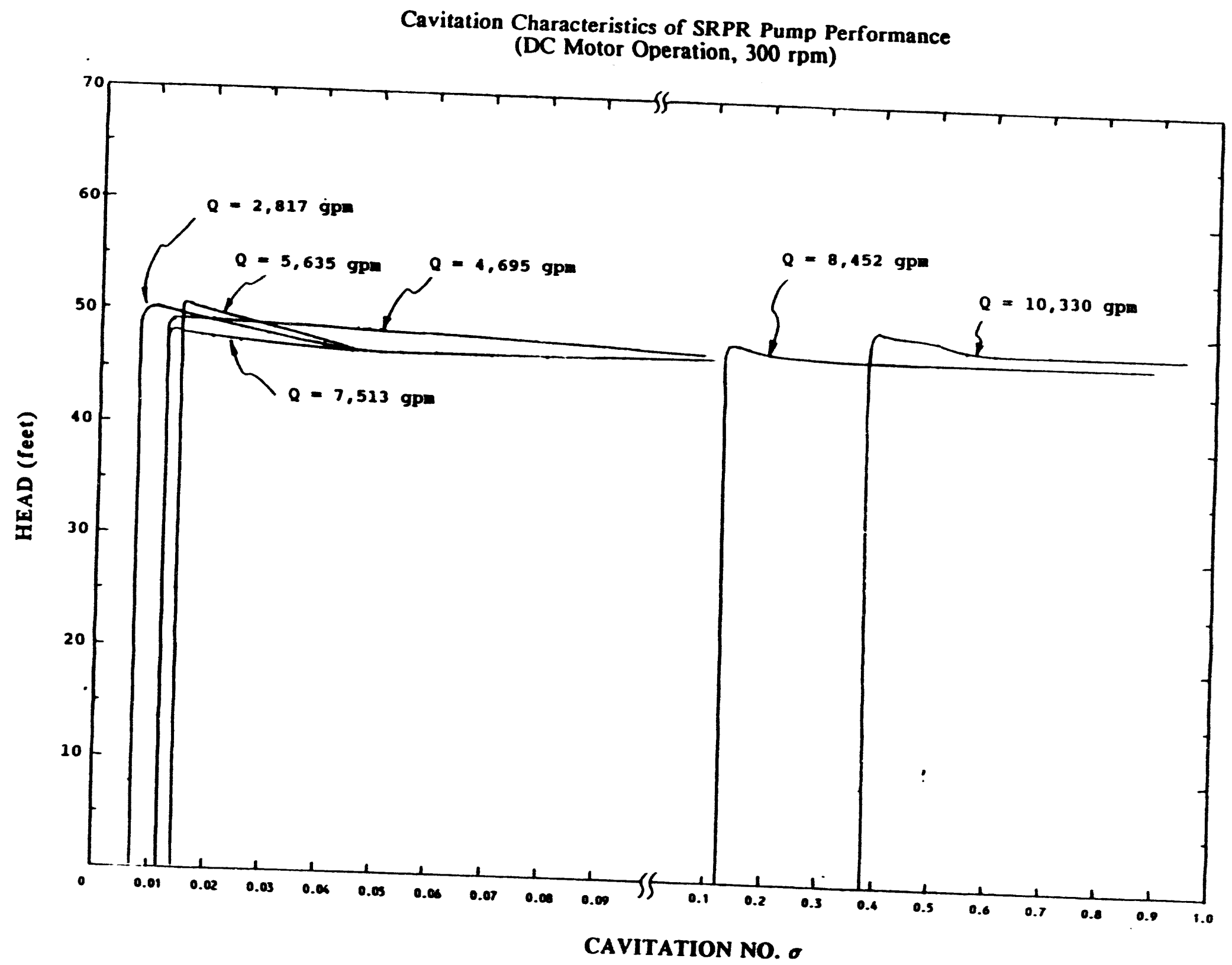


Figare 2.5.1

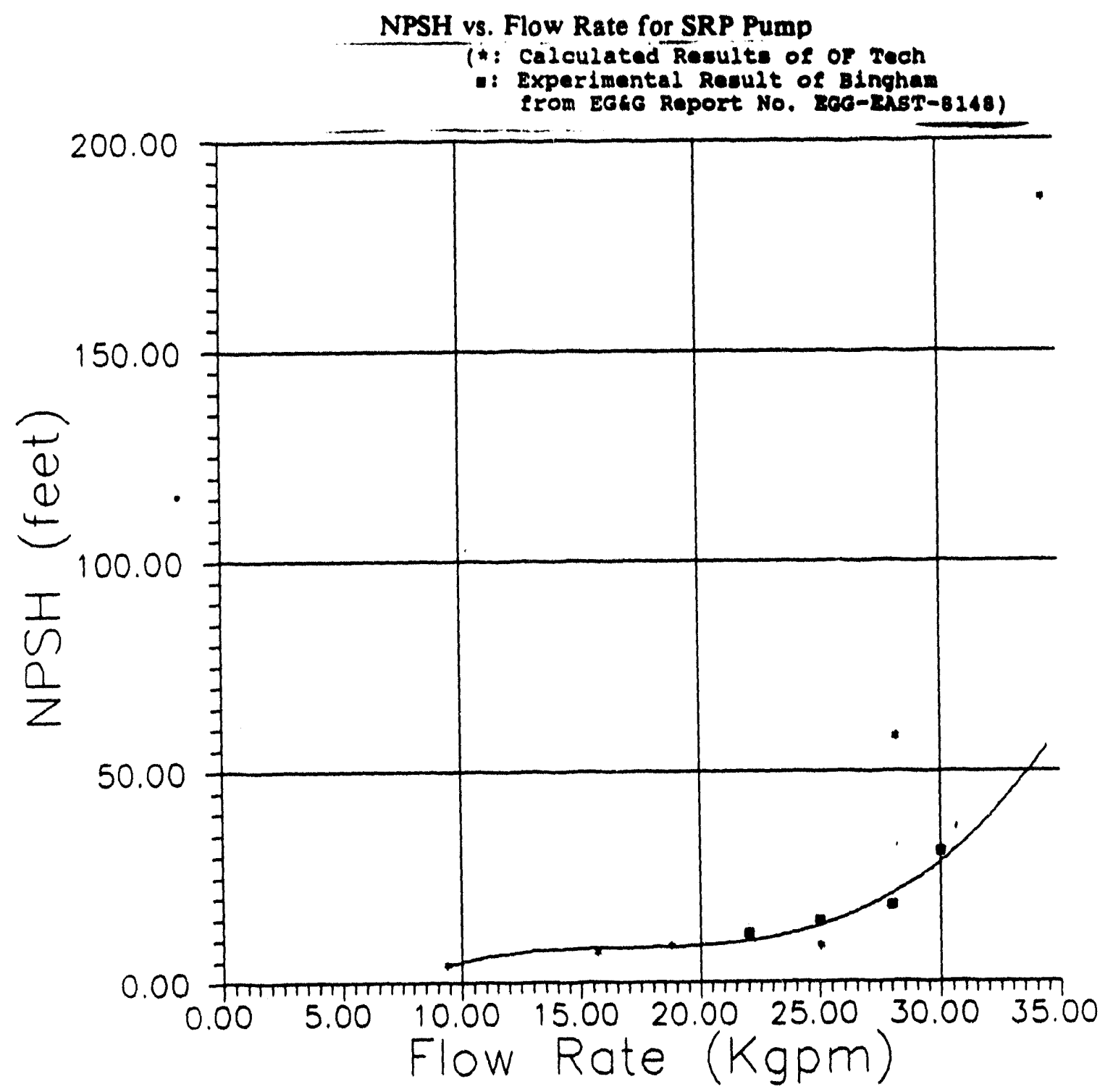


Figure 2.5.2

Difference in $\mathrm{NPSH}_{1}$ as a Function of Temperature

(from Figure F3 of EGG-EAST-8148)






\section{APPENDIX 2A \\ Explicit Expressions For Eans. (2,2,3-1) and (2,2,3-2)}

Substituting $\zeta_{1}$ together with Eqn. (2.2.3-) yields to the following expressions

$$
\begin{aligned}
& 0+0 \cdot i=\sqrt{\left(\zeta_{1}+b\right) / \zeta_{1}}\left[(1 / 2 \pi i) \int_{-\infty}^{b} 2 u / \sqrt{\left(\xi^{\prime}+b\right) / \xi^{\prime}} d \xi^{\prime} /\left(\xi^{\prime}-\zeta_{1}\right)+\right. \\
&(1 / 2 \pi i) \int_{-b^{0}}^{0} 2 v / \sqrt{\left(b-\xi^{\prime}\right) / \xi^{\prime}} d \xi^{\prime} /\left(\xi^{p}-\zeta_{1}\right)+ \\
&(1 / 2 \pi i) \int_{0}^{\infty} 2 u / \sqrt{\left(\xi^{\prime}+b\right) / \xi^{\prime}} d \xi^{\prime} /\left(\xi^{\prime}-\zeta_{1}\right) \\
&\left.+A \zeta_{1}+B\right]
\end{aligned}
$$

where $\zeta_{1}=e^{i(\pi / 2-w)}$. In order for the computations to be carried out, Eqn. $(2 A-1)$ should be separated into the real and imaginary parts as follows.

i) $\sqrt{\left(s_{1}+b\right) / s_{1}}$ :

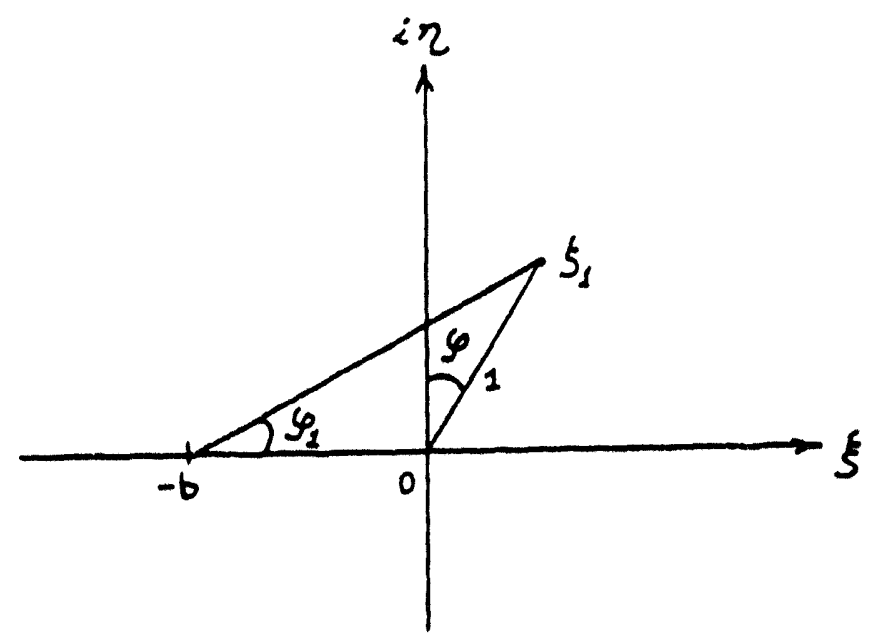

$$
\begin{aligned}
\zeta_{1}+b & =\left[(\sin w+b)^{2}+(\cos w)^{2}\right]^{1 / 2} e^{i \omega_{1}} \\
& =\left(1+2 b \sin w+b^{2}\right)^{1 / 2} e^{i w_{1}}
\end{aligned}
$$


where

$$
\begin{aligned}
& \tan w_{1}=\cos w /(\sin w+b) \\
& \zeta_{1}=e^{i(\pi / 2-w)} .
\end{aligned}
$$

Therefore,

$$
\begin{aligned}
& {\left[\left(\zeta_{1}+b\right) / \zeta_{1}\right]^{1 / 2}=R_{0}+i I_{0} } \\
& R_{0}=\left(1+2 b \sin w+b^{2}\right)^{1 / 4} \cos \left[\left(w_{1}+w-\pi / 2\right) / 2\right] \\
& I_{0}=\left(1+2 b \sin w+b^{2}\right)^{1 / 4} \sin \left[\left(w_{1}+w-\pi / 2\right) / 2\right] \\
& 1 /\left(\xi^{\prime}-\zeta_{1}\right): \\
& 1 /\left(\xi^{\prime}-\zeta_{1}\right)=R_{1}(\xi)+i I_{1}(\xi)
\end{aligned}
$$

ii) $1 /\left(\xi^{\prime}-\varsigma_{1}\right)$ :

where

$$
\begin{aligned}
& R_{1}(\xi)=\left(\xi^{\prime}-\sin \omega\right) /\left(\xi^{\prime 2}-2 \xi^{4} \sin \omega+1\right) \\
& I_{1}(\xi)=\cos w /\left(\xi^{\prime 2}-2 \xi^{\prime} \sin w+1\right)
\end{aligned}
$$

iii) A $\zeta_{1}+B$ :

$$
A \zeta_{1}+B=R_{2}+i I_{2}
$$

where

$$
\begin{aligned}
& R_{2}=A \sin w+B \\
& I_{2}=B \cos w
\end{aligned}
$$

Therefore, Eqn. $(2 A-1)$ is written

$$
0+0 \cdot i=\left(R_{0}+i I_{0}\right)\left[(-i / 2 \pi) \int_{-\infty}^{b} 2 u / \sqrt{\left(\xi^{\prime}+b\right) / \xi^{0}}\left(R_{1}\left(\xi^{\prime}\right)+i I_{1}\left(\xi^{\prime}\right)\right) d \xi^{\dagger}+\right.
$$




$$
\begin{aligned}
& (-i / 2 \pi) \int_{-b^{0}}^{0} 2 v / \sqrt{\left(b-\xi^{\prime}\right) / \xi^{\prime}}\left(R_{1}\left(\xi^{\prime}\right)+i I_{1}\left(\xi^{\prime}\right)\right) d \xi^{\prime}+ \\
& (-i / 2 \pi) \int_{0}^{\infty} 2 u / \sqrt{\left(\xi^{\prime}+b\right) / \xi^{\prime}}\left(R_{1}\left(\xi^{\prime}\right)+i I_{1}\left(\xi^{\prime}\right)\right) d \xi^{\prime} \\
& \left.+R_{2}+i I_{2}\right] .
\end{aligned}
$$

By defining the real and imaginary parts in the square brackets by $R_{\text {aum }}$ and $I_{\text {sum }}$, Eqn. $(2 A-1)$ is now reduced to the following two equations,

$$
\begin{aligned}
& R_{0} R_{\text {sum }}-I_{0} I_{\text {sum }}=0 \\
& R_{0} I_{\text {sum }}+I_{0} R_{\text {sum }}=0
\end{aligned}
$$

The closure condition of Eqn. (2.2.3-2) is also explicitly expressed in a manner similar to that used above.

$$
\begin{aligned}
& 0=\operatorname{Re}\left[W\left(\zeta_{2}\right) e^{-i \gamma}\right]=\operatorname{Re}\left(e ^ { - i \gamma } \left[\sqrt{\left(\zeta_{2}+b\right) / \zeta_{2}}(1 / 2 \pi i) \int_{-\infty}^{b} 2 u / \sqrt{\left(\xi^{\prime}+b\right) / \xi^{\prime}} d \xi^{\prime} /\left(\xi^{\prime}-\zeta_{2}\right)+\right.\right. \\
& (1 / 2 \pi i) \int_{-b}^{0} 2 v / \sqrt{\left(b-\xi^{\prime}\right) / \xi} d \xi /\left(\xi-\zeta_{2}\right)+ \\
& (1 / 2 \pi i) \int_{0}^{\infty} 2 u / \sqrt{\left(\xi^{p}+b\right) / \xi^{+}} d \xi^{*} /\left(\xi^{+}-\zeta_{2}\right) \\
& \left.\left.+A \zeta_{2}+B\right]\right)
\end{aligned}
$$

iv) $\sqrt{\left(5_{2}+b\right) / 5_{2}}$ :

$$
\sqrt{\left(\zeta_{2}+b\right) / \zeta_{2}}=R_{01}+i I_{01}
$$


where

$$
\begin{aligned}
& R_{01}=\left(a^{2}-2 a b \sin w+b^{2}\right)^{1 / 4} \cos w_{2} \\
& I_{01}=\left(a^{2}-2 a b \sin w+b^{2}\right)^{1 / 4} \sin w_{2}
\end{aligned}
$$

$\tan w_{2}=a \cos w /(b-a \sin w), b-a \sin w>0$

$$
\begin{array}{cc}
w_{2}=\pi / 2 & , b-a \sin w=0, \\
w_{2}=\pi-w_{2}^{\prime} & , b-a \sin w<0, \\
& \tan w_{2}^{\prime}-|a \cos w /(b-a \sin w)|
\end{array}
$$

v) $1 /\left(\xi^{4}-5_{2}\right)$ :

$1 /\left(\xi^{\prime}-s_{2}\right)=R_{8}(\xi)+i I_{8}(\xi)$

where

$$
\begin{aligned}
& R_{3}(\xi)=\left(\xi^{1}+a \sin w\right) /\left(\xi^{2}+a^{2}+2 a \xi^{4} \sin w\right) \\
& I_{3}(\xi)=a \cos w /\left(\xi^{2}+a^{2}+2 a \xi^{0} \sin w\right)
\end{aligned}
$$

vi) $\quad A S_{2}+B$ :

$A S_{2}+B=R_{4}+i I_{4}$

where

$$
\begin{aligned}
& R_{4}=-a \sin w+B \\
& I_{4}=a \cos w
\end{aligned}
$$


Now defining the real and imaginary parts in the square brackets in Eqn. $(2 A-4)$ by $R_{\text {oume }}$ and $l_{\text {aume' }}$ Eqn. $(2 A-4)$ becomes

$$
\begin{aligned}
& \left(R_{01} R_{\text {aume }}-I_{01} I_{\text {oume }}\right) \cos \gamma \\
& +\left(R_{01} R_{\text {aume }}+I_{01} I_{\text {sume }}\right) \sin \gamma=0
\end{aligned}
$$




\section{APPENDIX 2B}

\section{Derivation of the Carity Clogure Condition}

The cavity closure condition may be obtained by formally calculating the two streamlines which 80 through the leading edge of the blade and forcing these two streamlines going along the top and the bottom of the blade and cavity system to match at the end of the cavity. However, it is readily shown that this geometric condition is equivalent to that of having no source inside the cavity-body closure system. In terms of the perturbed velocities, i.e., $u$ and $v$, in the w-plane, this condition provides the condition of having zero net mass flow increase across the blade so that

$$
(u \cos \gamma-v \sin \gamma) \text { at the downotroum infinity }=0 \text {. }
$$

In the 5-plane, Eqd. (2B-1) is written

$$
\operatorname{Real}\left[W\left(\zeta_{2}\right) e^{-i \gamma}\right]=0
$$

A more explicit expression of this relationship has already been given in Appendix A. 
where the derivatives are taken along the are length " $\mathrm{g}$ " along the flow passage or the stream surface and " $A$ " denotes the cross section area of the blade passage normal to the direction of " $\mathrm{s}$ ". All other symbols are summarized in the section above (Section 3.2.1 Nomenclature).

The momentum equation for the gas bubble is written

$$
\frac{d}{d s}\left(\rho_{c} \propto w_{B}^{2} A\right)+C_{V m} \rho_{1} w_{s} \frac{d}{d s}\left(\left(w_{s}-w_{1}\right) \propto A\right)
$$

$$
--\frac{d p}{d s} \cdot a A+p_{8} a A r w^{2} \frac{d r}{d s}+\frac{C_{d}}{r_{8}} \cdot \frac{3}{8} \cdot p_{1} a A\left(w_{8}-w_{1}\right) \cdot\left|w_{8}-w_{1}\right|
$$

It should be noted that the second term on the left hand side of Equation (3.2.1-3) is the added (virtual) mass term, and the second and third terms on the right hand side are the centrifugal force term and the drag force term between the two phases acting on the bubble wall, respectively. The added mass (or virtual mass) coefficient $\mathrm{C}_{\mathrm{Vm}}$ is theoretically determined to be 0.5 for the case of the bubble having spherical shape (see Landau and Lifshitz (1959)).

On the other hand, the momentum equation for the two-phase flow mixture is provided

$$
\begin{aligned}
& \frac{d}{d s}\left(\rho_{B} \alpha w_{B}^{2} A\right)+\frac{d}{d s}\left[\rho_{1}(1-\alpha) w^{2} A\right] \\
& =-\frac{d p}{d s} \cdot A+\left[\rho_{B} \alpha+p_{1}(1-\alpha)\right] r w^{2} \frac{d r}{d s}
\end{aligned}
$$


Finally, the polytropic process for the aas phase is expressed by

$p / p_{g}^{\gamma}=$ constant.

\subsubsection{Solution Method-.--Homolosous Head (Task 3)}

There are five unknown parameters on the present equations, l.e., $\alpha_{,} p_{8}, w_{1}, w_{8}$ and $p$ for the five equations, Equations $(3.2 .1-1)$ to $(3.2 .1-5)$. The initial values for these unknown parameters are readily determined by selecting the flow rate, pump rotational speed, inlet void fraction and static pressure tosether with the pump geometry and the seometry of the Mow stream surface. These four nonlinear ordinary differential equations, $(3.2 .1-1)$ to $(3.2 .1-4)$, with one functional equation $(3.2 .1-5)$ can be solved by typical numerical method such as the Runge-Kutte method, the predictorcorrector method, etc. The accuracy of the numerical computations are tested by changing the number of control points covering the inlet to the exit of the pump. As the results of the computations, these five solution parameters at the exit of the pump are obtained and used to calculate various pump characteristics such as the head and the void fraction at the exit.

Two-Phase Flow Head of Pump (Task 3)

The head of the pump operating under the two-phase flow condition is defined from the energy balance between the inlet and the exit of the pump

$$
\begin{aligned}
H_{21}= & \frac{p_{2}-p_{1}}{p_{18} 8}(1-x)+\int_{1}^{2} \frac{d p}{p_{8} 8} \cdot x \\
& +\frac{1}{28}\left(v_{12}^{2}-v_{12}^{2}\right)(1-x)+\frac{1}{28}\left(v_{12}^{2}-v_{81}^{2}\right) \cdot x
\end{aligned}
$$


If the density of the gas phase should remain constant (i.e., for example, in the case of solid particle/water flow), then the above equation may be written

$$
\begin{aligned}
H_{20}= & \frac{p_{2}-p_{1}}{p_{1} 8}(1-x)+\left(\frac{p_{2}}{p_{82} 8}-\frac{p_{1}}{p_{81} 8}\right) \cdot x+ \\
& +\frac{1}{28}\left(v_{12}^{2}-v_{11}^{2}\right)(1-x)+\frac{1}{28}\left(v_{82}^{2}-v_{81}^{2}\right) \cdot x
\end{aligned}
$$

The formula for the head given in Equation (3.2.4-1a) can be reduced to the conventional form if $v_{11}$ $=v_{12}=v_{81}-v_{82}$ and $p_{81}=p_{s a}$. i.e., the assumption of the homogeneous flow and incompressibility,

$$
H_{10}=\frac{p_{2}-p_{1}}{p_{8}} \text {. }
$$

The complexity in the definition of the two-phase flow head stems from the following two factors:

i) the change of the gas density between the inlet and exit due to the compressibility, and

ii) the velocity slip between two-phases, i.e., $v_{18} \neq v_{8 g}$.

It is proved that the sum of the static head at the upstream (or inlet) of the pump between the liquid and the gas phases becomes that of the two-phase mixture flow, which is expressed in a more conventional form, i.e.,

$$
\frac{p_{1}}{p_{18}}(1-x)+\frac{p_{1}}{p_{81} 8} \times=\frac{p_{1}}{p_{2 \phi 1} 8}
$$


where

$$
p_{2 \phi 1}=\left(1-\alpha_{1}\right) \cdot p_{1}+\alpha_{1} \cdot p_{81}
$$

and the following relationships for $x$ and $(1-x)$ have been used for the derivation of the right hand side of Equation (3.2.4-4):

$$
\begin{aligned}
x & =m_{1} / m \\
1-x & =m_{1} / m \\
m & =m_{8}+m_{1} \\
m_{c} & =A\left(1-\alpha_{1}\right) w_{11} \rho_{1} \\
m_{1} & =A \alpha_{1} w_{11} \rho_{81} \\
w_{11} & =w_{s 1} .
\end{aligned}
$$

However, the sum of the downstream (or exit) static pressure is expressed somewhat different from the conventional expression, i.e., $p_{2} /\left(p_{2102} 8\right)$ even if $p_{8}=$ constant

$$
\frac{P_{2}}{P_{18}}(1-x)+\frac{P_{2}}{P_{82} 8} \times=\frac{P_{2}}{P_{242}^{*} 8}
$$

where

$$
\rho_{2,2} *=\frac{\left(1-\alpha_{2}\right) w_{12} \rho_{1}+\alpha_{2} w_{82} \rho_{82}}{\left(1-\alpha_{2}\right) w_{12}+\alpha_{2} w_{82} .}
$$

Of course, if $w_{12}=w_{82}$

$$
\rho_{2+2}^{*}=\left(1-\alpha_{1}\right) \rho_{1}+\alpha_{2} \rho_{c 2}=\rho_{2+2} \text {. }
$$

thus the total static head of the two-phase flow would be written 


$$
H_{2 \phi \text { conventional }}=\frac{p_{2}}{\rho_{2 \phi 2} \mathrm{~g}}-\frac{p_{1}}{\rho_{2 \phi 1} \mathrm{~g}}
$$

A similar argument also applies to the dynamic head part of Equation $(3.2 .4-1 \mathrm{a})$. If $v_{12}=v_{82} \equiv v_{2}$, then the dynamic head part would be written

$$
\text { Dynamic Head Part of Equation (3.2.4-1a) }=\frac{1}{2 g}\left(v_{2}^{2}-v_{1}^{2}\right) \text {. }
$$

It has been demonstrated that the head defined for the two-phase flow is clearly different from that of the single-phase flow case due to the existence of the slip between two-phases.

With the understanding of the facts described above, the head of the SRPR pump operating under the air/water two-phase flow conditions will be provided in the form of Equation (3.2.4-1). However, in order for the user to be able to perform various analyses of the pump or the subsequent hydraulic system, the detailed data at the exit of the pump are also necessary, including the void fraction $\alpha_{2}$, the relative flow speeds of liquid and gas, $w_{12}$ and $w_{z_{2}}$, and the static pressure $p_{2}$.

One of the important features of the SRPR pump system is that the inlet (or suction) and exit (or discharge) pipe diameters of the pump are different, i.e., 20" and 16". As has been explained above, even if these pipe diameters are the same, the liquid and gas ( or air in this case) flow velocities are different due to the slip. Due to this additional complexity, i.e., the difference in the pipe diameters of the SRPR pump system, it is readily seen that providing the so-called flange-to-flange head is meaningless. It should be noted that the present two-phase flow pump model only calculates the flow behavior between the inlet and exit of the pump and the head provided in the following section is only that between these two points. From the exit of the pump blade, these two-phase flow still needs to go through the diffuser section before reaching the discharge pipe section having the diameter of 16". The thermohydraulic calculations of the diffuse part of the pump are therefore left for the system analysis code in consideration of this section being one part of the primary coolant system. It is for this reason that the detailed data at the pump blade exit point are also provided as part of the present calculations. 
Nomenclature

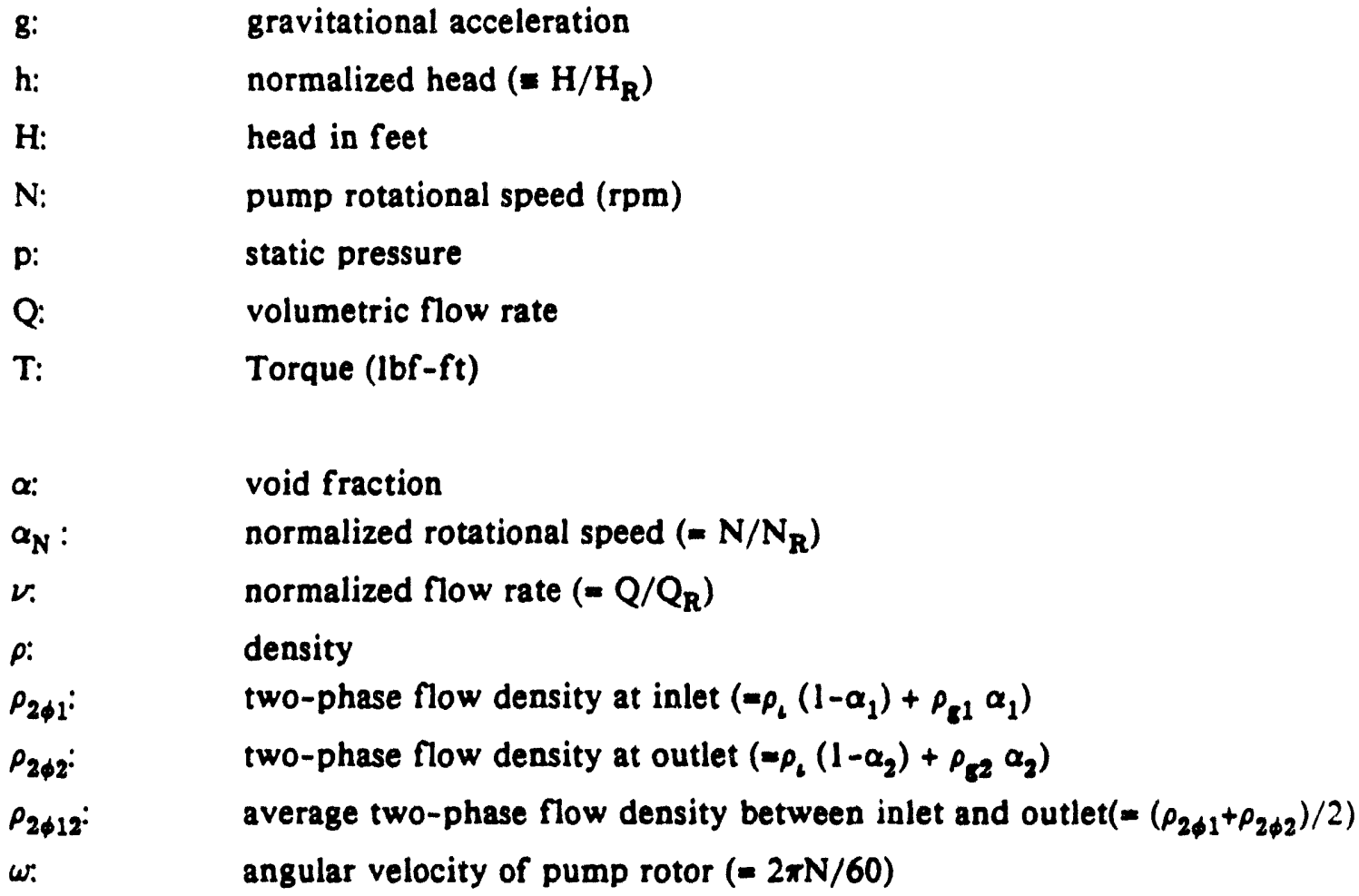

Subscriots:
8,l:
denotes the quantities related to the gas and liquid phases, respectively
1,2: demotes the quantities related to the inlet and exit of the pump, respectively
R: denotes the quantities at the pump rated condition
$2 \phi:$ two-phase flow quantities
1ф: single-phase flow quantities

The single-phase and two-two-phase torque will be written, respectively,

$$
\begin{aligned}
& T_{1 \phi} \omega=\left(H_{1 \phi}+\Delta H_{1 \phi}\right) Q_{\rho_{1 \phi}} 8 \\
& T_{2 \phi} \omega=\left(H_{2 \phi}+\Delta H_{1 \phi}\right) Q_{\rho_{2 \phi 12}} 8
\end{aligned}
$$


By eliminating $\Delta \mathrm{H}_{1 \phi}$ from these equations and dividing both sides by $H_{R}$ and $\omega$, one obtains

$$
T_{2 \phi} \omega /\left(\rho_{2 \phi 12} g Q H_{R}\right)-T_{1 \phi \omega} /\left(\rho_{1 \phi} g Q H_{R}\right)=h_{2 \phi}-h_{1 \phi} .
$$

Since $T_{1 \phi} \omega /\left(\rho_{1 \phi} g \mathrm{QH}_{R}\right)=h_{1 \phi} / \eta$ and the normalized torque is defined as $\beta_{2 \phi}=T_{2 \phi} \rho_{R} /\left(T_{R} \rho_{2 \phi 1}\right)$, the equation above now becomes

$$
\beta_{2 \phi}\left(T_{R} \omega / \rho_{2 \phi 12} B Q H_{R}\right)\left(\rho_{2 \phi 1} / \rho_{R}\right)=h_{2 \phi}-h_{1 \phi}+h_{1 \phi} / \eta \text {. }
$$

Use of $T_{R} \omega_{R} /\left(\rho_{R} g Q H_{R}\right)=1 / \eta_{R}$, rearrangement of the terms and division of both sides by $\alpha_{N}{ }^{2}$ will finally lead to the formula for the homologous torque

$$
\begin{aligned}
\beta_{2 \phi} / \alpha_{N}^{2} & =\left(\left(h_{2 \phi}-h_{1 \phi}\right)+h_{1 \phi} / \eta\right)\left(\rho_{2 \phi 12} / \rho_{2 \phi 1}\right) \\
& \bullet\left(\nu / \alpha_{N}\right) \eta_{R} / \alpha_{N}^{2} .
\end{aligned}
$$

The definitions of the various symbols used in this derivation are provide in the subsequent section. The two-phase flow homologous head in this equation has already been calculated for both the AC and DC operations and tabulated in the OF Technology Report No. 88-003 (AC Motor case) and 88007). These will be utilized for the present calculations. The two-phase flow average density will be calculated by using the pressure and void fraction data at exit of the pump which have also been provided in the reports mentioned above. The rest of the terms in Equation (3.2.5-1) are obtained from the single-phase flow performance of the pump.

The efficiency of the pump is provided in the report of E.I. du Pont, Savannah River Laboratory, NO. DPST $-88-777$ as follows:

$$
\begin{aligned}
& \eta=c_{1}+c_{2}(q / \omega)+c_{3}(q / \omega)^{2} \\
& c_{1}=.1321 \\
& c_{2}=2.980 \\
& c_{3}=-2.966 \\
& q=\text { flow rate in } \mathrm{ft}^{3} / \mathrm{sec} \\
& \omega=\text { rotational speed in radian } / \mathrm{sec} .
\end{aligned}
$$




\subsection{Numerical Calculations--- Without Diffuser (Task 3 and Task 9)}

\subsubsection{Parametric Range}

1) Pump

The SRPR pump is a double suction, double-volute centrifugal pump manufactured by Bingham Corp. having the rated flow rate of $25,000 \mathrm{gpm}(12,500 \mathrm{gpm} /$ volute) with the total head of 420 feet at the rated rotational speed of 1,000 rpm. The specific speed $N_{1}$ is 1,205 in the English units, indicating that the pump is a typical centrifugal pump:

$$
\begin{aligned}
N_{1} & =N Q^{1 / 2} / H^{3 / 4} \\
& =1,000 \times 12,500^{1 / 2} / 420^{3 / 4}=1,205
\end{aligned}
$$

The head vs. flow rate curve driven by the $\mathrm{AC}$ motor at $1,000 \mathrm{rpm}$ is given in Figure 3.3.1-1.

2) Line Static Pressure $\left(p_{1}\right)$

The following line static pressures were used for the present calculations:

$$
\begin{aligned}
& 0.05 \mathrm{~atm} \\
& 1.00 \mathrm{~atm} \\
& 2.00 \mathrm{~atm} \\
& 3.00 \mathrm{~atm}
\end{aligned}
$$

3) Flow Rate ( Q ) and Pump Rotational Speed (N)

Since the pump performance only depends on the ratio between the flow rate and the rotational speed, the range of these parameters is expressed in terms of the homologous flow parameter defined by $\nu$ $/ \alpha_{N}$ where 


$$
\begin{gathered}
\nu=Q / Q_{R} \\
\alpha_{N}=N / N_{R} .
\end{gathered}
$$

The range of the homologous parameter used for the calculations is

$$
\begin{array}{r}
\nu / \alpha_{N}= \\
0.4 \\
0.6 \\
0.8 \\
1.0 \\
1.2 \\
1.4
\end{array}
$$

With these homologous parameters, the range of the actual flow rate will be $10,000 \mathrm{gpm}$ to 35,000 gpm assuming the constant rotational speed (i.e., 1,000 rpm).

$$
\begin{aligned}
& \text { 4) Inlet Void Fraction }\left(\alpha_{1}\right) \\
& \quad \alpha_{1}=0.00 \text { to } 0.98
\end{aligned}
$$

\subsubsection{Results---Homologous Head (Without Diff user) (Task-3)}

Figures 3.3.2-1a to $1 \mathrm{~d}$ show the homologous head as the function of the inlet void fraction with the homologous flow parameter $\nu / \alpha_{N}$ ranging from 0.04 to 1.4 at the static pressure $p_{1}=3 \mathrm{~atm}, 2 \mathrm{~atm}$, $1 \mathrm{~atm}$ and $0.05 \mathrm{~atm}$, respectively.

Tables 3.3.2-1a to Id simply provide the homologous head data used for the figures above in terms of the numbers.

Tables 3.3.2-2 to 5 are the detailed pump hydraulic data as discussed in Section 3.2.4. Tables 3.3.2-2a to $2 \mathrm{~d}$ provide the void fraction at the exit of the pump blade for the same inlet static pressure range while Tables 3.3.2-3a to $3 \mathrm{~d}$ present the static pressure at the pump blade exit.

Tables 3.3.2-4a to $4 \mathrm{~d}$ and $3.3 .2-5 \mathrm{a}$ to $5 \mathrm{~d}$ provide the relative flow velocities of water and air at the

$$
3-12
$$


blade exit of the pump, respectively. These relative flow velocities in these tables were normalized by the pump blade tip speed $u_{2}$ at the rated pump rotational speed of $1000 \mathrm{rpm}$ :

$$
\begin{aligned}
u_{2 \text { at } 1000 \mathrm{rpm}} & =2 \pi N_{R} r_{2} / 60 \\
& =2 \pi \times 1000 \times 21^{\prime \prime} / 12 / 60 \\
& =183.26 \mathrm{ft} / \mathrm{sec}
\end{aligned}
$$

The absolute velocities of water and air at the exit of the pump blade will be readily calculated from the velocity triangle with the blade angle at the exit $\beta=17.071^{\circ}$ known (see the table at page $2-2$ of the interim report for Task 2).

\subsubsection{Results---Homologous Torque (Without Diffuser) (Task 9)}

The following parameters were used in the Formula given in Equation (3.2.5-1) for the AC motor operation:

$$
\begin{aligned}
& Q_{R}=25,000 \mathrm{gpm} \\
& H_{R}=420 \text { feet } \\
& \rho_{R} 8=66.6 \mathrm{lbm} / \mathrm{ft}^{3} \text { for the heavy water at } 1 \mathrm{~atm} \\
& T_{R}=16,963 \mathrm{lbf}-\mathrm{ft} \\
& \eta_{R}=87.8 \% \\
& \rho_{g 1} 8=0.00234 \times 32.17 \times p_{1} / 14.7 \mathrm{lbm} / \mathrm{ft}^{3} \\
& P_{1}=\text { line pressure at inlet in psi } \\
& \alpha_{N}=1 .(=1000 / 1000)
\end{aligned}
$$

Tables 3.3.2-6a to $6 \mathrm{~d}$ provide the homologous head as the function of the inlet void fraction with the homologous flow parameter $\nu / \alpha_{N}$ ranging from 0.4 to 1.4 at the static pressure $p_{1}=3 \mathrm{~atm}, 2 \mathrm{~atm}$, 1 atm and 0.05 atm for the $A C$ operation.

\subsection{Theoretical Basis---With Diffuser (Task 15)}

The diffuser section is mathematically treated in a manner similar to that for the pump blade section execpt that the centrifugal force is omitted from the momentum equations for both the liquid and gas 
phases. Although incorporation of this portion into the existing computer code requires a substantial effort from the viewpoints of controlling various parameters, change is rather straightforwad. The purpose of continuing the hydrodynamic computations through the diffuser portion is mainly to obtain the information of the detailed hydrodynamic parameters including the static pressure, liquid velocity, gas velocity and void fraction at the discharge flange. Unlike the single phase flow case, the Bernoulli's equation would not provide any such data since two phases slip in the diffuser seciton as well.

$$
\text { Numerical Calculations---With Diffuser (Task 15) }
$$

\subsection{1}

$$
\text { Parametric Range }
$$

1) Pump (The same as that of Section 3.3.1)

$$
\begin{array}{ll}
\text { Rated Flow Rate } \mathrm{Q}_{\mathrm{R}}: & 25,000 \mathrm{gpm}(12,500 \mathrm{gpm} / \text { volute) } \\
\text { Rated Head } \mathrm{H}_{\mathrm{R}}: & 402.9 \mathrm{feet} \text { (Experimental Interpolation) } \\
\text { Rotation Speed (AC Motor): } & 1,000 \mathrm{rpm}
\end{array}
$$

2) Line Static Pressure $\left(p_{1}\right)$

The following line static pressures were used for the present calculations:

$$
\begin{aligned}
& 0.5 \mathrm{~atm} \\
& 1.00 \mathrm{~atm} \\
& 2.00 \mathrm{~atm} \\
& 3.00 \mathrm{~atm}
\end{aligned}
$$

3) Flow Rate ( Q ) and Pump Rotational Speed (N)

Since the pump performance only depends on the ratio between the flow rate and the rotational speed. the range of these parameters is expressed in terms of the homologous flow parameter defined by $/ \alpha_{N}$ where 


$$
\begin{gathered}
\nu=Q / Q_{R} \\
\alpha_{N}=N / N_{R} .
\end{gathered}
$$

The range of the homologous parameter used for the calculations is

$$
\begin{aligned}
& \nu / \alpha_{N}=0.2 \\
& 0.4 \\
& 0.6 \\
& 0.8 \\
& 1.0 \\
& 1.2 \\
& 1.4
\end{aligned}
$$

With these homologous parameters, the range of the actual flow rate will be $5,000 \mathrm{gpm}$ to $35,000 \mathrm{gpm}$ assuming the constant rotational speed (i.e., 1,000 rpm).

4) Inlet Void Fraction $\left(\alpha_{1}\right)$

$$
\alpha_{1}=0.00 \text { to } 0.98
$$

Tables 3.5.2-1a to Id show the static pressure increase $\left(=p_{2}-p_{1}\right)$ (or decrease in some cases), liquid flow velocity $v_{12}$, gas flow velociy $v_{22}$ and void fraction $\alpha_{2}$ at the discharge flange section as the function of the inlet void fraction with the homologous flow parameter $\nu / \alpha_{N}$ ranging from 0.2 to 1.4 at the static pressure $p_{1}=3 \mathrm{~atm}, 2 \mathrm{~atm}, 1 \mathrm{~atm}$ and $0.5 \mathrm{~atm}$, respectively.

Babcock \& Wilcox, 1977, One third dcale air water pump program, Test program and pump performance, EPRI NP-135, July. 
Creare, Inc. 1982, Two-phase performance of scale models of a primary coolant pump, Block, J. A., Principal Investigator), EPRI NP-2578, September.

Furuya, O., 1985, An analytical model for prediction of two-phase (noncondensable) flow pump performance, J. of Fluids Eng., ASME, vol. 97, no.4, 419-429

Furuya, O., 1987, An analytical model for pump performance in condensable two-phase flows, EPRI NP-5529SP, November.

Landau, L.D. and Lifshitz, E.M., 1959, Fluid Mechanics, PERGAMON PRESS, London. 
Figure 3.3.1-1

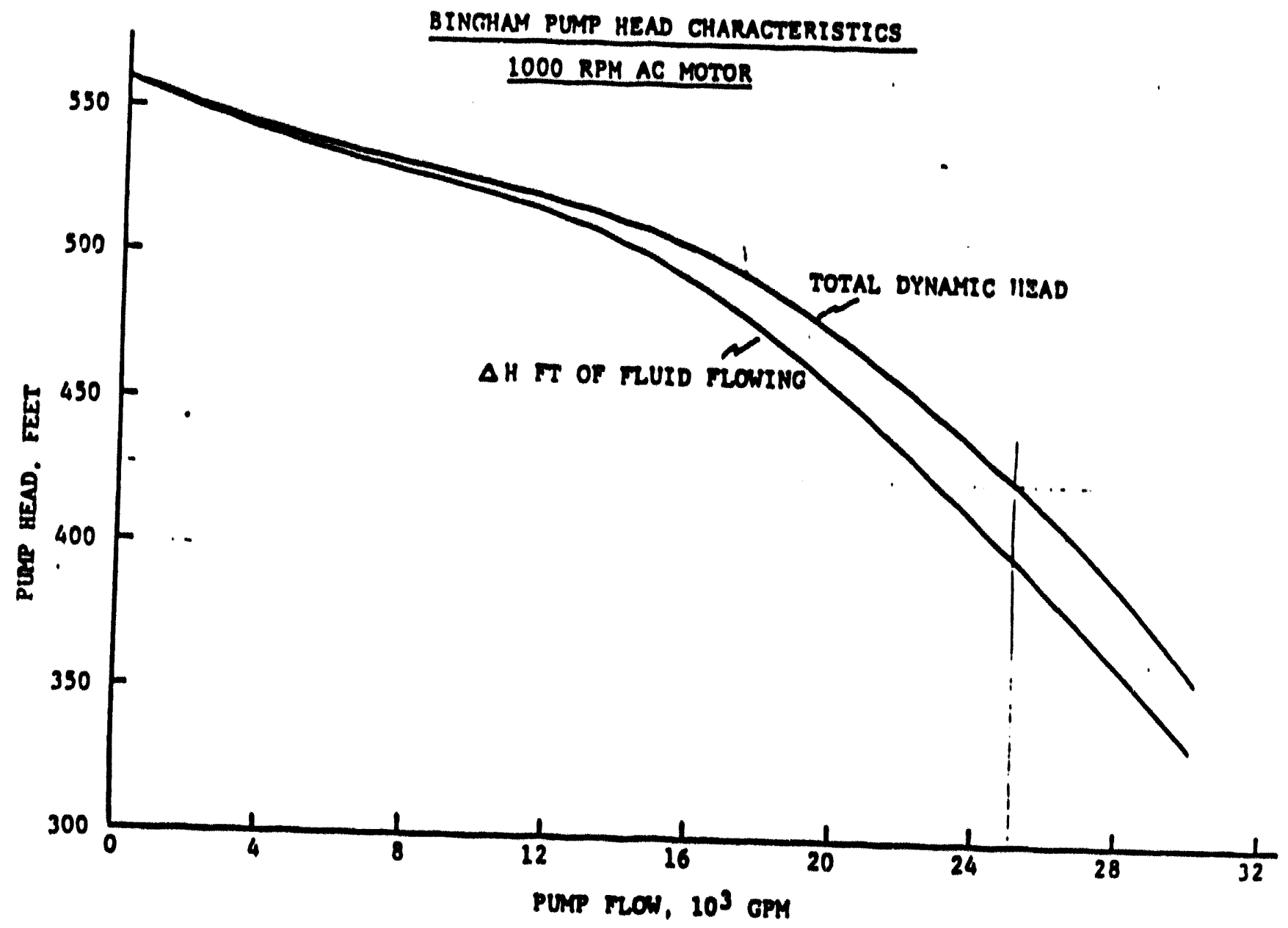


Figura 3.3.1-2

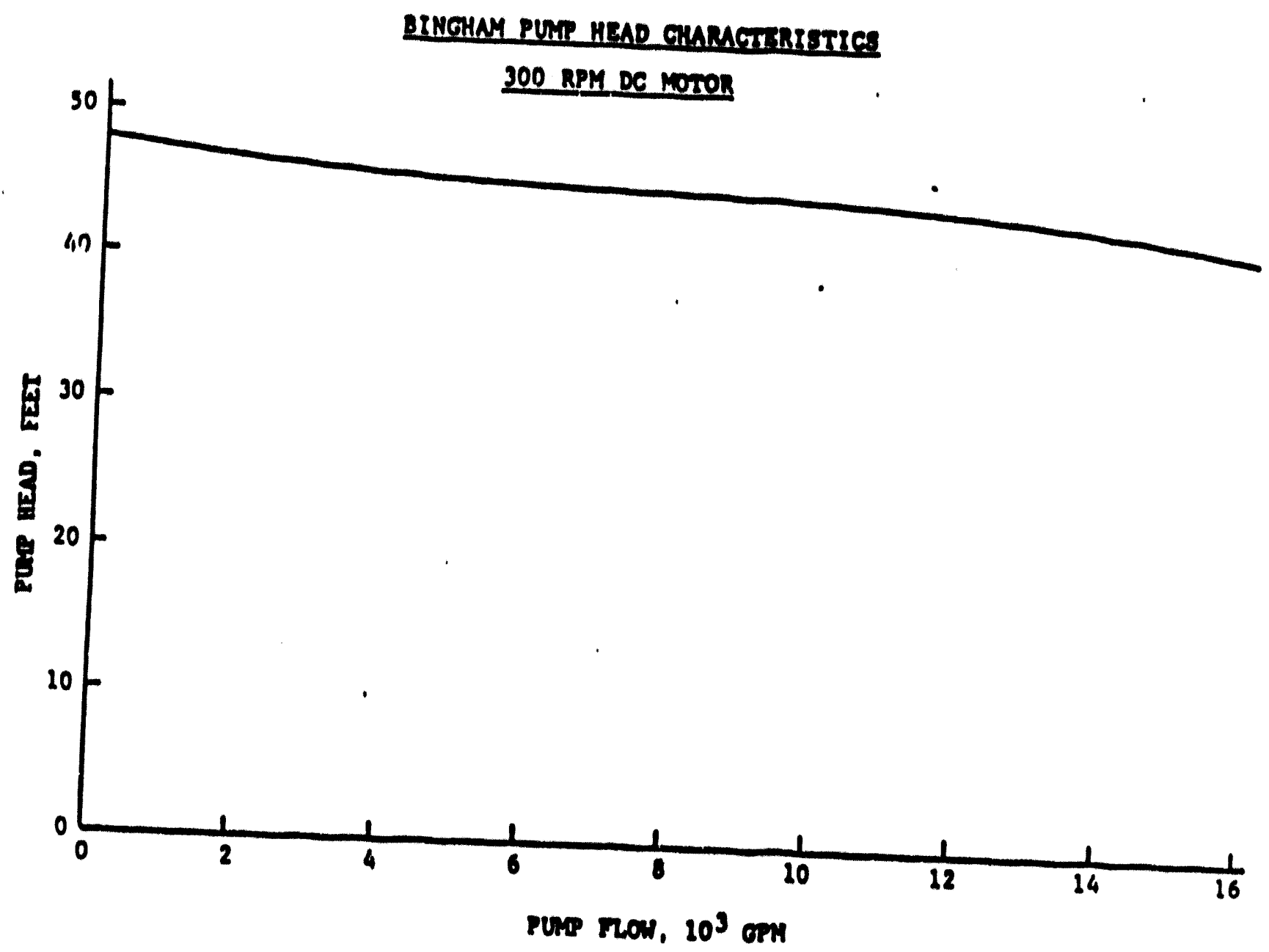


Figare 3.3.2-1a $\quad\left(p_{1}=3 \mathrm{ata}\right)$

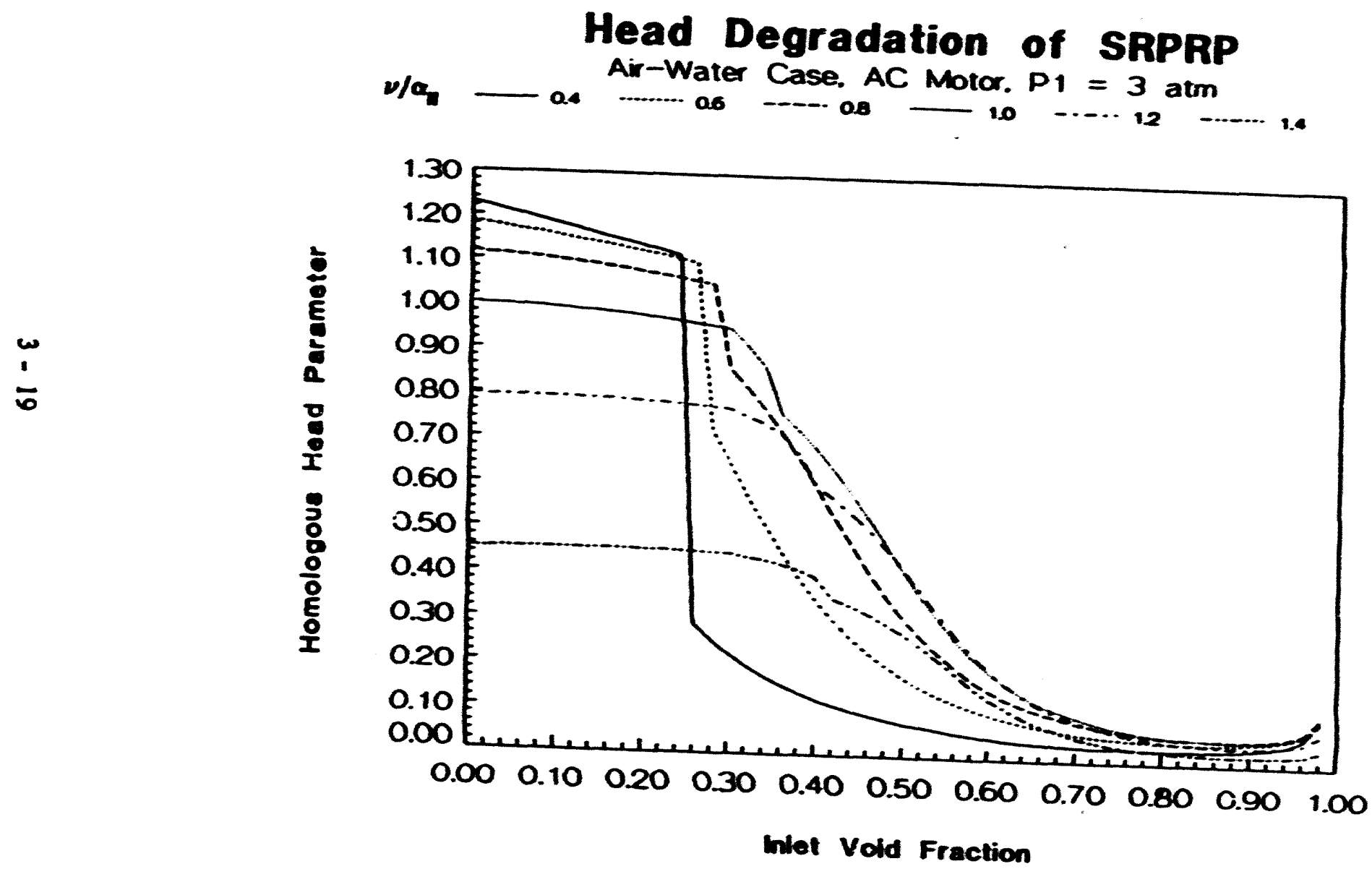


Flgure 3.3.2-1b $\quad\left(p_{1}=2 \mathrm{atb}\right)$

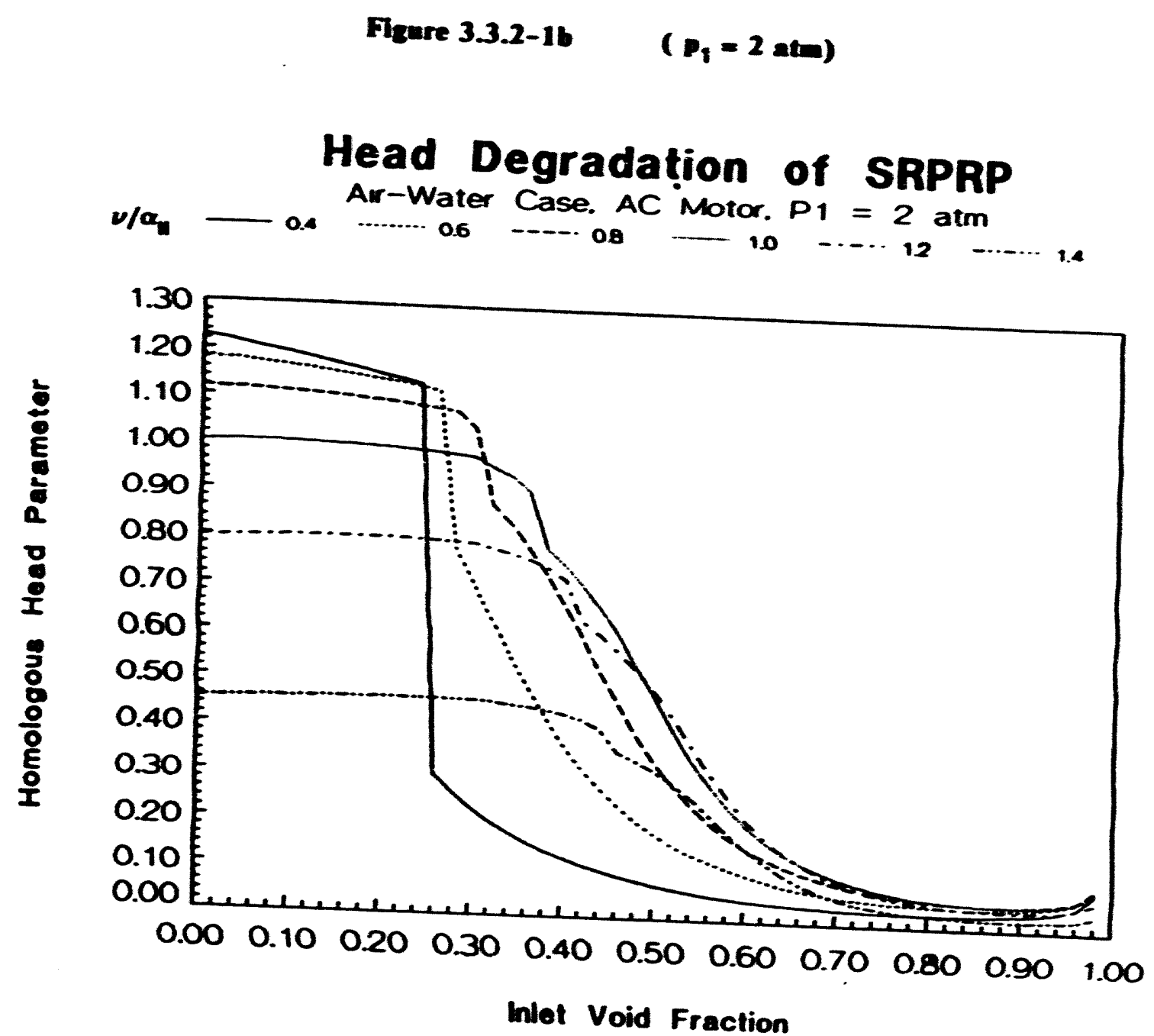




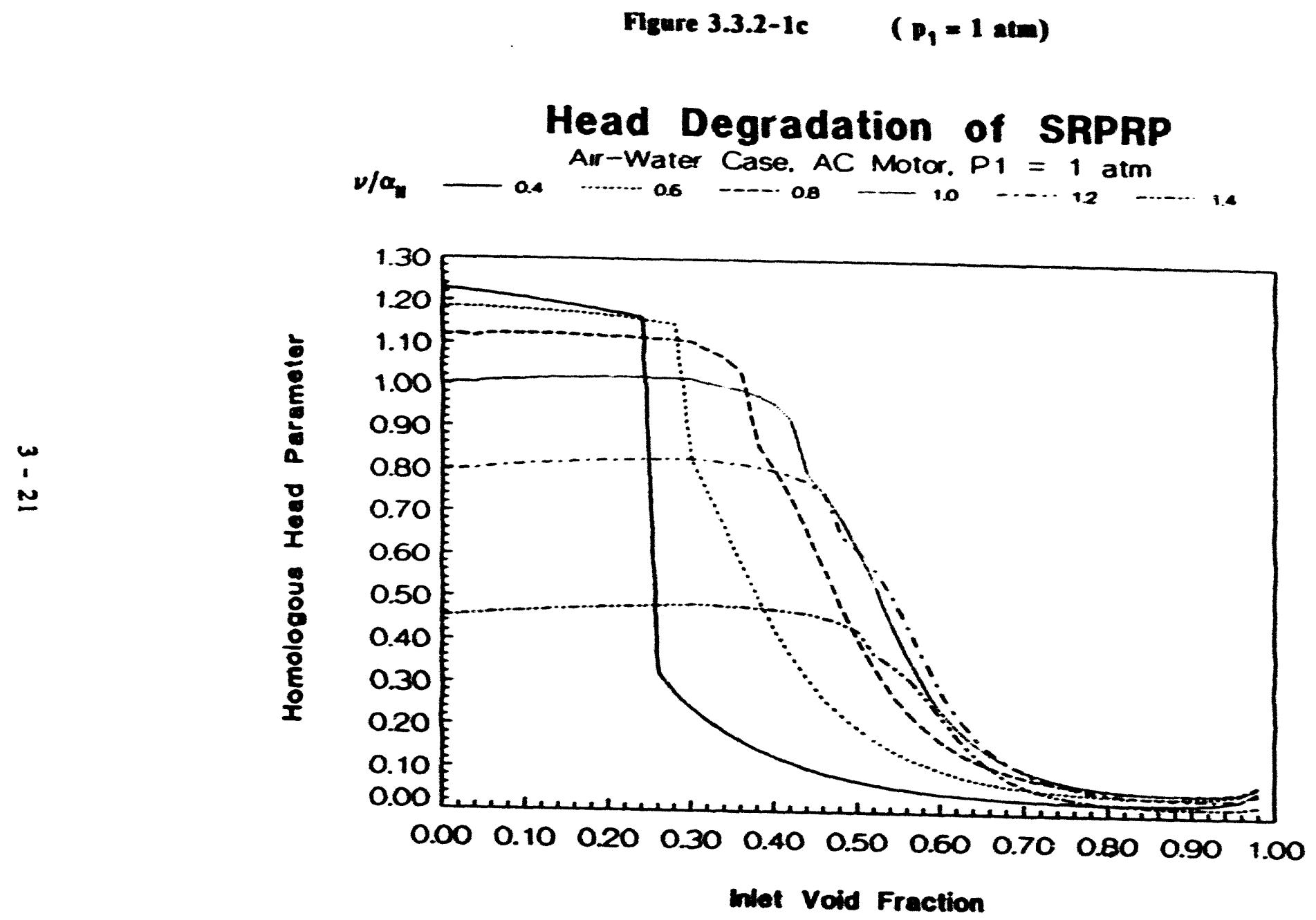


Figure 3.3.2-1d $\quad\left(p_{1}=0.05 \mathrm{~atm}\right)$

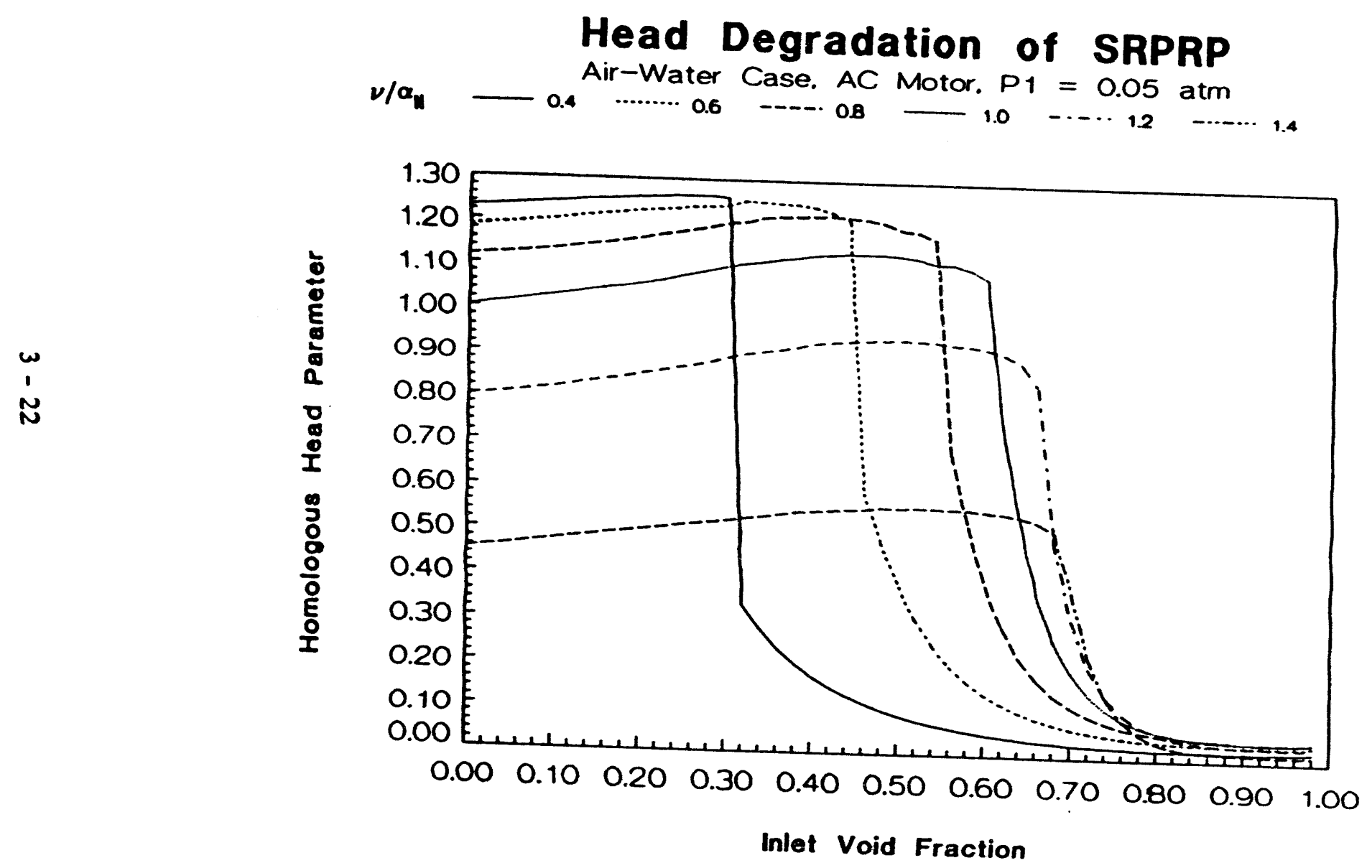


Table 3.3.2-1a $\quad\left(p_{1}=3 \mathrm{~atm}\right)$

Homologous Head Parameter vs. Inlet vold Fraction for Various Homologous Flow Parameters at P1 = 3 atm AC Motor Case

\begin{tabular}{|c|c|c|c|c|c|c|}
\hline \multirow{2}{*}{$\begin{array}{l}\text { Inlet } \\
\text { vold }\end{array}$} & \multicolumn{6}{|c|}{ Howologous Flow Paraneter } \\
\hline & 0.4 & 0.6 & 0.8 & 1.0 & 1.2 & 2.4 \\
\hline 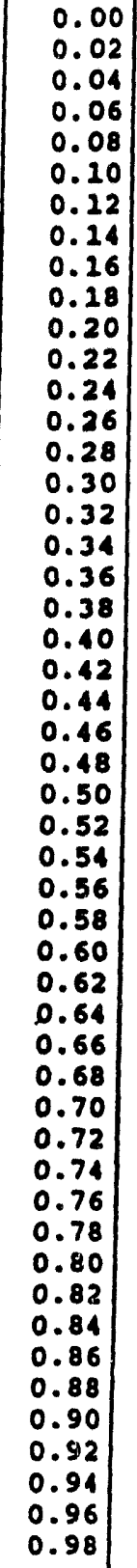 & $\begin{array}{l}1.230 \\
1.221 \\
1.212 \\
1.203 \\
1.193 \\
1.184 \\
1.175 \\
1.165 \\
1.155 \\
1.146 \\
1.236 \\
1.126 \\
1.215 \\
0.289 \\
0.254 \\
0.224 \\
0.198 \\
0.176 \\
0.157 \\
0.140 \\
0.125 \\
0.112 \\
0.101 \\
0.091 \\
0.082 \\
0.074 \\
0.068 \\
0.062 \\
0.057 \\
0.052 \\
0.048 \\
0.045 \\
0.042 \\
0.040 \\
0.038 \\
0.036 \\
0.035 \\
0.034 \\
0.033 \\
0.033 \\
0.033 \\
0.033 \\
0.033 \\
0.035 \\
0.036 \\
0.039 \\
0.043 \\
0.050 \\
0.065 \\
0.105\end{array}$ & $\begin{array}{l}1.186 \\
1.281 \\
1.175 \\
1.169 \\
1.164 \\
1.157 \\
1.251 \\
1.244 \\
1.138 \\
1.130 \\
1.223 \\
1.116 \\
1.208 \\
1.099 \\
0.723 \\
0.653 \\
0.584 \\
0.518 \\
0.456 \\
0.100 \\
0.350 \\
0.306 \\
0.267 \\
0.234 \\
0.205 \\
0.180 \\
0.159 \\
0.141 \\
0.125 \\
0.112 \\
0.100 \\
0.090 \\
0.082 \\
0.075 \\
0.069 \\
0.064 \\
0.060 \\
0.057 \\
0.054 \\
0.052 \\
0.051 \\
0.050 \\
0.050 \\
0.050 \\
0.051 \\
0.053 \\
0.056 \\
0.063 \\
0.074 \\
0.112 \\
\end{array}$ & $\begin{array}{l}1.119 \\
1.116 \\
1.113 \\
1.109 \\
1.106 \\
1.102 \\
1.097 \\
1.093 \\
1.088 \\
1.083 \\
1.077 \\
1.071 \\
1.065 \\
1.059 \\
1.051 \\
0.861 \\
0.820 \\
0.774 \\
0.722 \\
0.665 \\
0.603 \\
0.539 \\
0.476 \\
0.1417 \\
0.362 \\
0.314 \\
0.272 \\
0.235 \\
0.201 \\
0.178 \\
0.156 \\
0.137 \\
0.121 \\
0.108 \\
0.097 \\
0.088 \\
0.080 \\
0.074 \\
0.069 \\
0.065 \\
0.063 \\
0.060 \\
0.059 \\
0.059 \\
0.059 \\
0.060 \\
0.063 \\
0.068 \\
0.079 \\
0.111 \\
\end{array}$ & $\begin{array}{l}1.000 \\
0.999 \\
0.999 \\
0.997 \\
0.995 \\
0.993 \\
0.990 \\
0.987 \\
0.984 \\
0.981 \\
0.977 \\
0.973 \\
0.969 \\
0.964 \\
0.959 \\
0.952 \\
0.915 \\
0.868 \\
0.762 \\
0.727 \\
0.688 \\
0.642 \\
0.592 \\
0.536 \\
0.478 \\
0.429 \\
0.364 \\
0.313 \\
0.269 \\
0.230 \\
0.197 \\
0.270 \\
0.147 \\
0.128 \\
0.112 \\
0.100 \\
0.089 \\
0.081 \\
0.074 \\
0.068 \\
0.064 \\
0.061 \\
0.059 \\
0.058 \\
0.057 \\
0.058 \\
0.060 \\
0.064 \\
0.073 \\
0.102 \\
\end{array}$ & $\begin{array}{l}0.796 \\
0.795 \\
0.795 \\
0.795 \\
0.794 \\
0.794 \\
0.793 \\
0.791 \\
0.790 \\
0.788 \\
0.786 \\
0.784 \\
0.781 \\
0.778 \\
0.775 \\
0.771 \\
0.755 \\
0.740 \\
0.718 \\
0.675 \\
0.606 \\
0.578 \\
0.546 \\
0.510 \\
0.469 \\
0.423 \\
0.371 \\
0.325 \\
0.279 \\
0.238 \\
0.201 \\
0.171 \\
0.115 \\
0.123 \\
0.106 \\
0.092 \\
0.080 \\
0.071 \\
0.063 \\
0.058 \\
0.053 \\
0.050 \\
0.047 \\
0.046 \\
0.045 \\
0.045 \\
0.047 \\
0.050 \\
0.056 \\
0.076\end{array}$ & $\begin{array}{l}0.453 \\
0.453 \\
0.454 \\
0.454 \\
0.455 \\
0.455 \\
0.455 \\
0.455 \\
0.454 \\
0.451 \\
0.453 \\
0.453 \\
0.452 \\
0.450 \\
0.449 \\
0.447 \\
0.441 \\
0.435 \\
0.427 \\
0.316 \\
0.100 \\
0.353 \\
0.338 \\
0.320 \\
0.300 \\
0.277 \\
0.251 \\
0.222 \\
0.292 \\
0.163 \\
0.137 \\
0.115 \\
0.095 \\
0.080 \\
0.067 \\
0.056 \\
0.048 \\
0.041 \\
0.036 \\
0.032 \\
0.029 \\
0.026 \\
0.025 \\
0.023 \\
0.023 \\
0.023 \\
0.023 \\
0.025 \\
0.028 \\
0.039\end{array}$ \\
\hline
\end{tabular}


Table 3.3.2-1b $\quad\left(P_{1}=2\right.$ atm $)$

Homologous Head Parameter ve. Inlet Vold Fraction for Various Homologous flow parameters at P1 $=2$ atm AC Motor Case

\begin{tabular}{|c|c|c|c|c|c|c|}
\hline \multirow{2}{*}{$\begin{array}{l}\text { Inlet } \\
\text { vold }\end{array}$} & \multicolumn{6}{|c|}{ Homologous Flow Parameter } \\
\hline & 0.4 & 0.6 & 0.8 & 1.0 & 1.2 & 1.4 \\
\hline $\begin{array}{l}0.00 \\
0.02 \\
0.04 \\
0.06 \\
0.08 \\
0.10 \\
0.12 \\
0.14 \\
0.16 \\
0.18 \\
0.20 \\
0.22 \\
0.24 \\
0.26 \\
0.28 \\
0.30 \\
0.32 \\
0.34 \\
0.34 \\
0.36 \\
0.38 \\
0.40 \\
0.42 \\
0.44 \\
0.46 \\
0.48 \\
0.50 \\
0.50 \\
0.52 \\
0.54 \\
0.56\end{array}$ & $\begin{array}{l}1.230 \\
1.223 \\
1.216 \\
1.208 \\
1.201 \\
1.193 \\
1.185 \\
1.177 \\
1.168 \\
1.160 \\
1.131 \\
1.142 \\
1.132 \\
0.298 \\
0.261 \\
0.229 \\
0.202 \\
0.179 \\
0.159 \\
0.111 \\
0.126 \\
0.112 \\
0.101 \\
0.090 \\
0.082 \\
0.074 \\
0.067 \\
0.061 \\
0.056 \\
0.051 \\
0.047 \\
0.044 \\
0.041 \\
0.039 \\
0.037 \\
0.035 \\
0.033 \\
0.032 \\
0.031 \\
0.031 \\
0.030 \\
0.030 \\
0.031 \\
0.031 \\
0.033 \\
0.031 \\
0.037 \\
0.043 \\
0.053 \\
0.085\end{array}$ & $\begin{array}{l}1.286 \\
1.180 \\
1.179 \\
1.174 \\
1.270 \\
1.166 \\
1.161 \\
1.156 \\
1.250 \\
1.145 \\
1.139 \\
1.133 \\
1.126 \\
1.119 \\
0.782 \\
0.708 \\
0.633 \\
0.560 \\
0.491 \\
0.428 \\
0.371 \\
0.322 \\
0.279 \\
0.243 \\
0.211 \\
0.185 \\
0.162 \\
0.142 \\
0.126 \\
0.112 \\
0.100 \\
0.090 \\
0.081 \\
0.074 \\
0.068 \\
0.063 \\
0.059 \\
0.055 \\
0.053 \\
0.051 \\
0.049 \\
0.048 \\
0.047 \\
0.047 \\
0.048 \\
0.049 \\
0.051 \\
0.056 \\
0.065 \\
0.094\end{array}$ & $\begin{array}{l}1.129 \\
1.118 \\
1.116 \\
1.211 \\
1.112 \\
1.110 \\
1.107 \\
1.104 \\
1.101 \\
1.097 \\
1.094 \\
1.090 \\
1.085 \\
1.080 \\
1.074 \\
1.040 \\
0.877 \\
0.836 \\
0.787 \\
0.731 \\
0.668 \\
0.598 \\
0.527 \\
0.458 \\
0.394 \\
0.338 \\
0.289 \\
0.247 \\
0.213 \\
0.183 \\
0.159 \\
0.139 \\
0.122 \\
0.108 \\
0.097 \\
0.087 \\
0.079 \\
0.073 \\
0.068 \\
0.064 \\
0.061 \\
0.058 \\
0.057 \\
0.056 \\
0.056 \\
0.056 \\
0.058 \\
0.062 \\
0.070 \\
0.095\end{array}$ & $\begin{array}{l}1.000 \\
1.001 \\
1.002 \\
1.002 \\
1.002 \\
1.001 \\
1.000 \\
0.999 \\
0.997 \\
0.996 \\
0.994 \\
0.991 \\
0.989 \\
0.986 \\
0.982 \\
0.977 \\
0.957 \\
0.938 \\
0.907 \\
0.787 \\
0.752 \\
0.710 \\
0.661 \\
0.605 \\
0.541 \\
0.473 \\
0.407 \\
0.346 \\
0.292 \\
0.247 \\
0.209 \\
0.178 \\
0.152 \\
0.231 \\
0.114 \\
0.100 \\
0.089 \\
0.080 \\
0.073 \\
0.067 \\
0.063 \\
0.059 \\
0.057 \\
0.055 \\
0.055 \\
0.055 \\
0.056 \\
0.059 \\
0.066 \\
0.087\end{array}$ & $\begin{array}{l}0.796 \\
0.796 \\
0.798 \\
0.799 \\
0.800 \\
0.801 \\
0.801 \\
0.801 \\
0.801 \\
0.801 \\
0.802 \\
0.800 \\
0.799 \\
0.797 \\
0.795 \\
0.793 \\
0.783 \\
0.774 \\
0.763 \\
0.747 \\
0.722 \\
0.633 \\
0.605 \\
0.572 \\
0.533 \\
0.487 \\
0.433 \\
0.376 \\
0.319 \\
0.267 \\
0.222 \\
0.185 \\
0.154 \\
0.229 \\
0.109 \\
0.093 \\
0.081 \\
0.071 \\
0.063 \\
0.057 \\
0.052 \\
0.048 \\
0.046 \\
0.044 \\
0.043 \\
0.043 \\
0.044 \\
0.046 \\
0.052 \\
0.067\end{array}$ & $\begin{array}{l}0.453 \\
0.454 \\
0.456 \\
0.457 \\
0.458 \\
0.459 \\
0.460 \\
0.461 \\
0.462 \\
0.462 \\
0.463 \\
0.463 \\
0.463 \\
0.463 \\
0.462 \\
0.461 \\
0.459 \\
0.453 \\
0.449 \\
0.443 \\
0.435 \\
0.424 \\
0.406 \\
0.358 \\
0.340 \\
0.319 \\
0.293 \\
0.263 \\
0.228 \\
0.492 \\
0.159 \\
0.130 \\
0.206 \\
0.086 \\
0.071 \\
0.058 \\
0.049 \\
0.042 \\
0.036 \\
0.031 \\
0.028 \\
0.026 \\
0.024 \\
0.022 \\
0.022 \\
0.021 \\
0.022 \\
0.023 \\
0.025 \\
0.034\end{array}$ \\
\hline
\end{tabular}


Table 3.3.2-1c $\quad\left(p_{q}=1 \mathrm{~atm}\right)$

Homologous Head Parameter ve. Inlet Vold Fraction for Varioue Homologous Flow Parameters at PI = 1 atm AC Motor Case

\begin{tabular}{|c|c|c|c|c|c|c|}
\hline \multirow{2}{*}{$\begin{array}{r}\text { Inlet } \\
\text { void }\end{array}$} & \multicolumn{6}{|c|}{ Homologous Flow Parameter } \\
\hline & 0.4 & 0.6 & 0.8 & 2.0 & 1.2 & 1.4 \\
\hline $\begin{array}{l}0.00 \\
0.02 \\
0.04 \\
0.06 \\
0.08 \\
0.20 \\
0.12 \\
0.24 \\
0.16 \\
0.18 \\
0.20 \\
0.22 \\
0.24 \\
0.26 \\
0.28 \\
0.30 \\
0.32 \\
0.34 \\
0.36 \\
0.38 \\
0.38 \\
0.40 \\
0.42 \\
0.44 \\
0.46 \\
0.48 \\
0.50 \\
0.52 \\
0.54 \\
0.56 \\
0.58 \\
0.60 \\
0.62 \\
0.64 \\
0.65\end{array}$ & $\begin{array}{l}1.230 \\
1.226 \\
1.221 \\
1.217 \\
1.212 \\
1.207 \\
1.201 \\
1.195 \\
1.189 \\
1.283 \\
1.176 \\
1.170 \\
1.162 \\
0.323 \\
0.279 \\
0.243 \\
0.213 \\
0.287 \\
0.265 \\
0.146 \\
0.129 \\
0.125 \\
0.102 \\
0.091 \\
0.082 \\
0.074 \\
0.067 \\
0.060 \\
0.055 \\
0.050 \\
0.046 \\
0.043 \\
0.040 \\
0.037 \\
0.035 \\
0.033 \\
0.032 \\
0.030 \\
0.029 \\
0.028 \\
0.028 \\
0.028 \\
0.028 \\
0.028 \\
0.028 \\
0.029 \\
0.031 \\
0.034 \\
0.042 \\
0.063\end{array}$ & $\begin{array}{l}1.186 \\
1.185 \\
1.184 \\
1.182 \\
1.180 \\
1.178 \\
1.176 \\
1.174 \\
1.171 \\
1.168 \\
1.164 \\
1.161 \\
1.156 \\
1.152 \\
1.146 \\
0.825 \\
0.747 \\
0.664 \\
0.580 \\
0.501 \\
0.429 \\
0.366 \\
0.313 \\
0.267 \\
0.230 \\
0.198 \\
0.171 \\
0.149 \\
0.230 \\
0.115 \\
0.101 \\
0.090 \\
0.081 \\
0.074 \\
0.067 \\
0.062 \\
0.057 \\
0.054 \\
0.051 \\
0.048 \\
0.047 \\
0.045 \\
0.044 \\
0.044 \\
0.044 \\
0.044 \\
0.046 \\
0.048 \\
0.054 \\
0.073\end{array}$ & $\begin{array}{l}1.119 \\
1.120 \\
1.121 \\
1.122 \\
1.122 \\
1.122 \\
1.122 \\
1.122 \\
1.121 \\
1.120 \\
1.219 \\
1.118 \\
1.116 \\
1.114 \\
1.111 \\
1.107 \\
1.086 \\
1.068 \\
1.031 \\
0.861 \\
0.805 \\
0.737 \\
0.657 \\
0.570 \\
0.184 \\
0.406 \\
0.339 \\
0.284 \\
0.238 \\
0.201 \\
0.171 \\
0.146 \\
0.127 \\
0.111 \\
0.098 \\
0.087 \\
0.079 \\
0.072 \\
0.066 \\
0.062 \\
0.059 \\
0.056 \\
0.054 \\
0.053 \\
0.052 \\
0.052 \\
0.053 \\
0.055 \\
0.060 \\
0.076\end{array}$ & $\begin{array}{l}1.000 \\
1.004 \\
1.007 \\
1.009 \\
1.011 \\
1.013 \\
1.015 \\
1.016 \\
1.017 \\
1.018 \\
1.029 \\
1.019 \\
1.019 \\
1.018 \\
1.018 \\
1.016 \\
1.007 \\
0.999 \\
0.991 \\
0.978 \\
0.959 \\
0.922 \\
0.793 \\
0.747 \\
0.689 \\
0.617 \\
0.532 \\
0.145 \\
0.366 \\
0.299 \\
0.241 \\
0.201 \\
0.167 \\
0.140 \\
0.119 \\
0.103 \\
0.090 \\
0.080 \\
0.072 \\
0.066 \\
0.061 \\
0.057 \\
0.055 \\
0.053 \\
0.052 \\
0.051 \\
0.052 \\
0.053 \\
0.057 \\
0.070\end{array}$ & $\begin{array}{l}0.796 \\
0.798 \\
0.802 \\
0.805 \\
0.808 \\
0.811 \\
0.814 \\
0.816 \\
0.819 \\
0.821 \\
0.822 \\
0.821 \\
0.825 \\
0.826 \\
0.827 \\
0.827 \\
0.826 \\
0.819 \\
0.815 \\
0.809 \\
0.802 \\
0.791 \\
0.775 \\
0.749 \\
0.651 \\
0.615 \\
0.569 \\
0.511 \\
0.439 \\
0.361 \\
0.290 \\
0.231 \\
0.181 \\
0.148 \\
0.221 \\
0.100 \\
0.081 \\
0.072 \\
0.063 \\
0.056 \\
0.051 \\
0.047 \\
0.044 \\
0.042 \\
0.041 \\
0.040 \\
0.040 \\
0.042 \\
0.044 \\
0.054\end{array}$ & $\begin{array}{l}0.453 \\
0.455 \\
0.457 \\
0.461 \\
0.464 \\
0.466 \\
0.468 \\
0.471 \\
0.473 \\
0.475 \\
0.476 \\
0.478 \\
0.480 \\
0.481 \\
0.482 \\
0.483 \\
0.483 \\
0.480 \\
0.479 \\
0.477 \\
0.474 \\
0.470 \\
0.465 \\
0.457 \\
0.446 \\
0.428 \\
0.372 \\
0.349 \\
0.329 \\
0.279 \\
0.230 \\
0.182 \\
0.140 \\
0.208 \\
0.084 \\
0.067 \\
0.053 \\
0.044 \\
0.037 \\
0.032 \\
0.028 \\
0.025 \\
0.023 \\
0.022 \\
0.020 \\
0.020 \\
0.020 \\
0.020 \\
0.022 \\
0.027\end{array}$ \\
\hline
\end{tabular}


Table 3.3.2-1d

$\left(p_{1}=0.05 \mathrm{~atm}\right)$

Homologous Head Parameter vs. Inlet Vold Fraction for Varlous Homologous Flow Parameters at $\mathrm{P}_{1}=0.05$ atm AC Motor Case

\begin{tabular}{|c|c|c|c|c|c|c|}
\hline \multirow{2}{*}{$\begin{array}{l}\text { Inlet } \\
\text { vold }\end{array}$} & \multicolumn{6}{|c|}{ Homologous Flow Paraneter } \\
\hline & 0.4 & 0.6 & 0.8 & 1.0 & 1.2 & 1.4 \\
\hline $\begin{array}{l}0.00 \\
0.02 \\
0.04 \\
0.06 \\
0.08 \\
0.10 \\
0.12 \\
0.14 \\
0.16 \\
0.18 \\
0.20 \\
0.22 \\
0.24 \\
0.26 \\
0.28 \\
0.30 \\
0.32 \\
0.34 \\
0.36 \\
0.38 \\
0.40 \\
0.42 \\
0.44 \\
0.46 \\
0.48 \\
0.50 \\
0.52 \\
0.54 \\
0.56 \\
0.58 \\
0.60 \\
0.62 \\
0.64 \\
0.66 \\
0.68 \\
0.70 \\
0.72 \\
0.74 \\
0.76 \\
0.78 \\
0.80 \\
0.82 \\
0.84 \\
0.86 \\
0.88 \\
0.90 \\
0.92 \\
0.94 \\
0.96 \\
0.98\end{array}$ & $\begin{array}{l}1.230 \\
1.232 \\
1.234 \\
1.237 \\
1.240 \\
1.243 \\
1.246 \\
1.249 \\
1.251 \\
1.254 \\
1.256 \\
1.258 \\
1.260 \\
1.261 \\
1.261 \\
1.258 \\
0.334 \\
0.279 \\
0.237 \\
0.204 \\
0.176 \\
0.152 \\
0.132 \\
0.125 \\
0.100 \\
0.088 \\
0.077 \\
0.068 \\
0.061 \\
0.054 \\
0.049 \\
0.044 \\
0.040 \\
0.036 \\
0.034 \\
0.032 \\
0.023 \\
0.029 \\
0.028 \\
0.026 \\
0.025 \\
0.024 \\
0.024 \\
0.023 \\
0.022 \\
0.023 \\
0.023 \\
0.023 \\
0.025\end{array}$ & 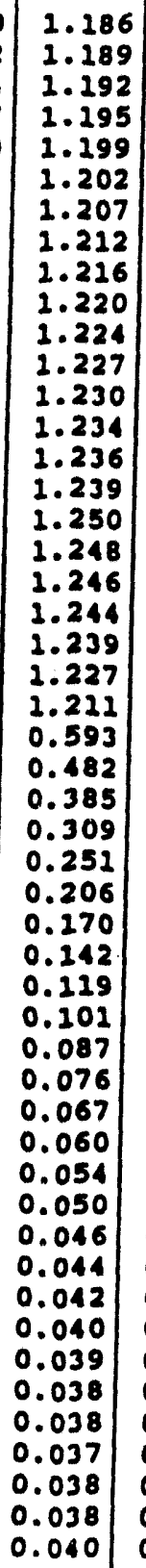 & $\begin{array}{l}1.119 \\
1.122 \\
1.125 \\
1.128 \\
1.132 \\
1.136 \\
1.142 \\
1.148 \\
1.152 \\
1.159 \\
1.165 \\
1.172 \\
1.178 \\
1.185 \\
1.192 \\
1.201 \\
1.204 \\
1.213 \\
1.216 \\
1.218 \\
1.219 \\
1.220 \\
1.218 \\
1.215 \\
1.208 \\
1.194 \\
1.190 \\
1.173 \\
0.688 \\
0.524 \\
0.391 \\
0.297 \\
0.229 \\
0.180 \\
0.144 \\
0.117 \\
0.097 \\
0.082 \\
0.071 \\
0.064 \\
0.058 \\
0.054 \\
0.051 \\
0.049 \\
0.047 \\
0.046\end{array}$ & $\begin{array}{l}1.000 \\
1.007 \\
1.011 \\
1.017 \\
1.022 \\
1.028 \\
1.033 \\
1.040 \\
1.047 \\
1.052 \\
1.059 \\
1.066 \\
1.075 \\
1.083 \\
1.092 \\
1.100 \\
1.107 \\
1.122 \\
1.129 \\
1.123 \\
1.129 \\
1.132 \\
1.135 \\
1.135 \\
1.135 \\
1.132 \\
1.127 \\
1.116 \\
1.115 \\
1.104 \\
1.083 \\
0.743 \\
0.541 \\
0.375 \\
0.268 \\
0.197 \\
0.148 \\
0.114 \\
0.091 \\
0.075 \\
0.065 \\
0.058 \\
0.053 \\
0.050 \\
0.048 \\
0.045 \\
0.045 \\
0.047\end{array}$ & $\begin{array}{l}0.796 \\
0.800 \\
0.804 \\
0.809 \\
0.824 \\
0.819 \\
0.826 \\
0.832 \\
0.838 \\
0.843 \\
0.852 \\
0.858 \\
0.866 \\
0.872 \\
0.880 \\
0.890 \\
0.899 \\
0.905 \\
0.920 \\
0.928 \\
0.927 \\
0.931 \\
0.934 \\
0.937 \\
0.939 \\
0.940 \\
0.939 \\
0.937 \\
0.931 \\
0.929 \\
0.923 \\
0.912 \\
0.892 \\
0.839 \\
0.494 \\
0.316 \\
0.210 \\
0.145 \\
0.204 \\
0.078 \\
0.061 \\
0.050 \\
0.044 \\
0.036\end{array}$ & $\begin{array}{l}0.453 \\
0.458 \\
0.462 \\
0.467 \\
0.472 \\
0.477 \\
0.482 \\
0.487 \\
0.492 \\
0.496 \\
0.500 \\
0.505 \\
0.510 \\
0.514 \\
0.518 \\
0.523 \\
0.528 \\
0.532 \\
0.538 \\
0.543 \\
0.546 \\
0.549 \\
0.552 \\
0.554 \\
0.557 \\
0.558 \\
0.559 \\
0.559 \\
0.558 \\
0.557 \\
0.554 \\
0.551 \\
0.545 \\
0.535 \\
0.516 \\
0.381 \\
0.236 \\
0.141 \\
0.089 \\
0.058 \\
0.040 \\
0.030 \\
0.024 \\
0.021 \\
0.019 \\
0.018 \\
0.018\end{array}$ \\
\hline
\end{tabular}


Table 3.3.2-2a

$\left(p_{1}=3 \mathrm{~atm}\right)$

Diecharge Vold Fraction vs. Inlet Vold Fraction for Various Homologous Flow Parameters at P1 - 3 atm AC Motor Case

\begin{tabular}{|c|c|c|c|c|c|c|}
\hline \multirow{2}{*}{$\begin{array}{l}\text { Inlet } \\
\text { vold } \\
\text { Iraction }\end{array}$} & \multicolumn{6}{|c|}{ Homologous Flow Paramete } \\
\hline & 0.4 & 0.6 & 0.8 & 1.0 & 1.2 & 1.4 \\
\hline $\begin{array}{l}0.00 \\
0.02 \\
0.04 \\
0.06 \\
0.08 \\
0.10 \\
0.12 \\
0.14 \\
0.16 \\
0.18 \\
0.20 \\
0.22 \\
0.24 \\
0.26 \\
0.28 \\
0.30 \\
0.32 \\
0.34 \\
0.36 \\
0.38 \\
0.40 \\
0.42 \\
0.44 \\
0.46 \\
0.48 \\
0.50 \\
0.52 \\
0.54 \\
0.56 \\
0.58 \\
0.60 \\
0.62 \\
0.64 \\
0.66 \\
0.68 \\
0.70 \\
0.72 \\
0.74\end{array}$ & $\begin{array}{l}0.00 \\
0.01 \\
0.03 \\
0.04 \\
0.06 \\
0.07 \\
0.09 \\
0.10 \\
0.22 \\
0.24 \\
0.26 \\
0.17 \\
0.19 \\
0.84 \\
0.86 \\
0.87 \\
0.87 \\
0.88 \\
0.89 \\
0.90 \\
0.90 \\
0.91 \\
0.91 \\
0.92 \\
0.92 \\
0.92 \\
0.93 \\
0.93 \\
0.94 \\
0.94 \\
0.94 \\
0.94 \\
0.95 \\
0.95 \\
0.95 \\
0.96 \\
0.96 \\
0.96 \\
0.97 \\
0.97 \\
0.97 \\
0.97 \\
0.98\end{array}$ & $\begin{array}{l}0.00 \\
0.01 \\
0.02 \\
0.03 \\
0.04 \\
0.05 \\
0.07 \\
0.08 \\
0.09 \\
0.11 \\
0.12 \\
0.14 \\
0.15 \\
0.17 \\
0.57 \\
0.62 \\
0.67 \\
0.71 \\
0.75 \\
0.77 \\
0.80 \\
0.82 \\
0.83 \\
0.85 \\
0.86 \\
0.87 \\
0.88 \\
0.89 \\
0.90 \\
0.90 \\
0.91 \\
0.92 \\
0.92 \\
0.93 \\
0.94 \\
0.94 \\
0.94 \\
0.95 \\
0.95 \\
0.96 \\
0.96 \\
0.97 \\
0.97 \\
0.97 \\
0.98 \\
0.98 \\
0.99 \\
0.99 \\
0.99 \\
1.00\end{array}$ & $\begin{array}{l}0.00 \\
0.00 \\
0.02 \\
0.03 \\
0.04 \\
0.05 \\
0.06 \\
0.07 \\
0.08 \\
0.09 \\
0.11 \\
0.12 \\
0.13 \\
0.15 \\
0.16 \\
0.37 \\
0.41 \\
0.46 \\
0.51 \\
0.56 \\
0.62 \\
0.66 \\
0.70 \\
0.74 \\
0.77 \\
0.79 \\
0.81 \\
0.83 \\
0.85 \\
0.86 \\
0.87 \\
0.88 \\
0.89 \\
0.90 \\
0.91 \\
0.92 \\
0.93 \\
0.93 \\
0.94 \\
0.94 \\
0.95 \\
0.95 \\
0.96 \\
0.96 \\
0.97 \\
0.98 \\
0.98 \\
0.99 \\
0.99 \\
1.00\end{array}$ & $\begin{array}{l}0.00 \\
0.00 \\
0.02 \\
0.03 \\
0.03 \\
0.04 \\
0.05 \\
0.06 \\
0.08 \\
0.09 \\
0.10 \\
0.11 \\
0.12 \\
0.14 \\
0.15 \\
0.17 \\
0.19 \\
0.21 \\
0.38 \\
0.42 \\
0.46 \\
0.51 \\
0.56 \\
0.61 \\
0.65 \\
0.70 \\
0.73 \\
0.76 \\
0.79 \\
0.81 \\
0.83 \\
0.85 \\
0.86 \\
0.87 \\
0.88 \\
0.90 \\
0.90 \\
0.91 \\
0.92 \\
0.93 \\
0.94 \\
0.94 \\
0.95 \\
0.96 \\
0.96 \\
0.97 \\
0.98 \\
0.98 \\
0.99 \\
0.99\end{array}$ & $\begin{array}{l}0.00 \\
0.00 \\
0.02 \\
0.02 \\
0.03 \\
0.04 \\
0.05 \\
0.06 \\
0.07 \\
0.08 \\
0.09 \\
0.10 \\
0.12 \\
0.13 \\
0.14 \\
0.16 \\
0.27 \\
0.19 \\
0.21 \\
0.25 \\
0.38 \\
0.42 \\
0.16 \\
0.51 \\
0.56 \\
0.60 \\
0.65 \\
0.69 \\
0.73 \\
0.76 \\
0.79 \\
0.81 \\
0.83 \\
0.85 \\
0.86 \\
0.88 \\
0.89 \\
0.90 \\
0.91 \\
0.92 \\
0.93 \\
0.93 \\
0.94 \\
0.95 \\
0.96 \\
0.96 \\
0.97 \\
0.98 \\
0.99 \\
0.99\end{array}$ & $\begin{array}{l}0.00 \\
0.00 \\
0.02 \\
0.02 \\
0.03 \\
0.04 \\
0.05 \\
0.06 \\
0.07 \\
0.08 \\
0.09 \\
0.10 \\
0.12 \\
0.13 \\
0.14 \\
0.15 \\
0.17 \\
0.18 \\
0.20 \\
0.22 \\
0.24 \\
0.37 \\
0.41 \\
0.44 \\
0.49 \\
0.53 \\
0.58 \\
0.63 \\
0.67 \\
0.71 \\
0.75 \\
0.77 \\
0.80 \\
0.82 \\
0.84 \\
0.86 \\
0.87 \\
0.88 \\
0.89 \\
0.90 \\
0.92 \\
0.93 \\
0.93 \\
0.94 \\
0.95 \\
0.96 \\
0.97 \\
0.98 \\
0.98 \\
0.99\end{array}$ \\
\hline
\end{tabular}


Table 3.3.2-2b

$\left(p_{1}=2 \mathrm{~atm}\right)$

Discharge Void Fraction vs. Inlet Vold Fraction for Varlous Homologous Flow Parameters at PI - 2 atm AC Motor Case

\begin{tabular}{|c|c|c|c|c|c|c|}
\hline \multirow{2}{*}{$\begin{array}{l}\text { Inlet } \\
\text { vold } \\
\text { eraction }\end{array}$} & \multicolumn{6}{|c|}{ Homologous Flow Paramete } \\
\hline & 0.4 & 0.6 & 0.8 & 2.0 & 2.2 & 2.4 \\
\hline $\begin{array}{l}\quad 0.00 \\
0.02 \\
0.04 \\
0.06 \\
0.08 \\
0.10 \\
0.22 \\
0.14 \\
0.16 \\
0.18 \\
0.20 \\
0.22 \\
0.24 \\
0.26 \\
0.28 \\
0.30 \\
0.32 \\
0.34 \\
0.34 \\
0.36 \\
0.38 \\
0.40 \\
0.12 \\
0.44\end{array}$ & $\begin{array}{l}0.00 \\
0.01 \\
0.02 \\
0.03 \\
0.05 \\
0.06 \\
0.07 \\
0.09 \\
0.20 \\
0.12 \\
0.13 \\
0.15 \\
0.16 \\
0.84 \\
0.85 \\
0.86 \\
0.87 \\
0.88 \\
0.89 \\
0.90 \\
0.90 \\
0.91 \\
0.91 \\
0.92 \\
0.92 \\
0.93 \\
0.93 \\
0.94 \\
0.94 \\
0.94 \\
0.94 \\
0.95 \\
0.95 \\
0.95 \\
0.96 \\
0.96 \\
0.96 \\
0.97 \\
0.97 \\
0.97 \\
0.98 \\
0.98 \\
0.98 \\
0.98 \\
0.99 \\
0.99 \\
0.99 \\
0.99 \\
1.00 \\
1.00\end{array}$ & 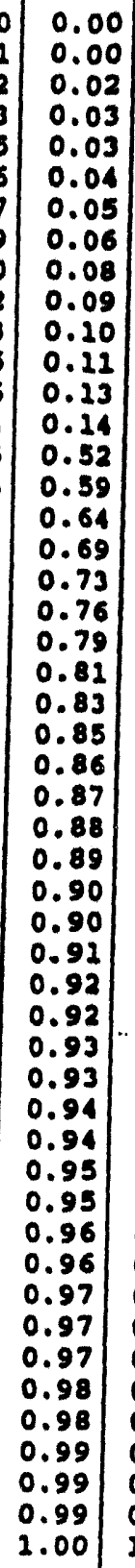 & $\begin{array}{l}0.00 \\
0.00 \\
0.01 \\
0.02 \\
0.03 \\
0.04 \\
0.05 \\
0.06 \\
0.07 \\
0.08 \\
0.09 \\
0.10 \\
0.11 \\
0.12 \\
0.14 \\
0.25 \\
0.35 \\
0.40 \\
0.45 \\
0.51 \\
0.57 \\
0.62 \\
0.68 \\
0.72 \\
0.75 \\
0.78 \\
0.81 \\
0.83 \\
0.85 \\
0.86 \\
0.87 \\
0.88 \\
0.89 \\
0.90 \\
0.91 \\
0.92 \\
0.92 \\
0.93 \\
0.94 \\
0.94 \\
0.95 \\
0.95 \\
0.96 \\
0.96 \\
0.97 \\
0.98 \\
0.98 \\
0.99 \\
0.99 \\
1.00\end{array}$ & $\begin{array}{l}0.00 \\
0.00 \\
0.01 \\
0.02 \\
0.03 \\
0.04 \\
0.04 \\
0.05 \\
0.06 \\
0.07 \\
0.08 \\
0.09 \\
0.10 \\
0.12 \\
0.12 \\
0.14 \\
0.15 \\
0.17 \\
0.19 \\
0.36 \\
0.40 \\
0.45 \\
0.50 \\
0.56 \\
0.62 \\
0.66 \\
0.71 \\
0.75 \\
0.78 \\
0.82 \\
0.83 \\
0.84 \\
0.86 \\
0.87 \\
0.88 \\
0.89 \\
0.90 \\
0.91 \\
0.92 \\
0.93 \\
0.94 \\
0.94 \\
0.95 \\
0.96 \\
0.96 \\
0.97 \\
0.98 \\
0.98 \\
0.99 \\
0.99 \\
0.99\end{array}$ & $\begin{array}{l}0.00 \\
0.00 \\
0.01 \\
0.02 \\
0.03 \\
0.03 \\
0.04 \\
0.05 \\
0.06 \\
0.07 \\
0.08 \\
0.08 \\
0.09 \\
0.10 \\
0.12 \\
0.13 \\
0.14 \\
0.16 \\
0.27 \\
0.29 \\
0.21 \\
0.36 \\
0.40 \\
0.44 \\
0.19 \\
0.55 \\
0.60 \\
0.66 \\
0.70 \\
0.71 \\
0.78 \\
0.80 \\
0.82 \\
0.84 \\
0.86 \\
0.87 \\
0.89 \\
0.90 \\
0.91 \\
0.92 \\
0.93 \\
0.93 \\
0.94 \\
0.95 \\
0.96 \\
0.96 \\
0.97 \\
0.98 \\
0.99 \\
0.99 \\
0.99 \\
0.94\end{array}$ & $\begin{array}{l}0.00 \\
0.00 \\
0.01 \\
0.01 \\
0.02 \\
0.03 \\
0.03 \\
0.01 \\
0.05 \\
0.05 \\
0.06 \\
0.07 \\
0.08 \\
0.09 \\
0.10 \\
0.11 \\
0.12 \\
0.13 \\
0.25 \\
0.16 \\
0.28 \\
0.19 \\
0.21 \\
0.23 \\
0.38 \\
0.42 \\
0.16 \\
0.51 \\
0.57 \\
0.63 \\
0.68 \\
0.72 \\
0.76 \\
0.79 \\
0.81 \\
0.83 \\
0.85 \\
0.87 \\
0.88 \\
0.89 \\
0.90 \\
0.92 \\
0.93 \\
0.93 \\
0.94 \\
0.95 \\
0.96 \\
0.99 \\
0.98 \\
0.98 \\
0.99\end{array}$ \\
\hline
\end{tabular}


Table 3.3.2-2c $\quad\left(p_{1}=1\right.$ atm $)$

Dlscharge Vold Fraction vo. Inlet Vold Fraction for various Homologous flow Parametere at PI - 1 atm AC Motor Case

\begin{tabular}{|c|c|c|c|c|c|c|}
\hline \multirow{2}{*}{$\begin{array}{l}\text { Inlet } \\
\text { Vold }\end{array}$} & \multicolumn{6}{|c|}{ Howologous Flow Paramete } \\
\hline & 0.4 & 0.6 & 0.8 & 2.0 & 2.2 & 1.4 \\
\hline $\begin{array}{l}0.00 \\
0.02 \\
0.04 \\
0.06 \\
0.08 \\
0.10 \\
0.12 \\
0.14 \\
0.16 \\
0.18 \\
0.20 \\
0.22 \\
0.24 \\
0.24 \\
0.26 \\
0.28 \\
0.30 \\
0.32 \\
0.34 \\
0.36 \\
0.36 \\
0.38 \\
0.40 \\
0.42\end{array}$ & $\begin{array}{l}0.00 \\
0.00 \\
0.02 \\
0.02 \\
0.03 \\
0.04 \\
0.05 \\
0.06 \\
0.07 \\
0.08 \\
0.10 \\
0.11 \\
0.12 \\
0.83 \\
0.85 \\
0.86 \\
0.87 \\
0.88 \\
0.89 \\
0.89 \\
0.90 \\
0.91 \\
0.91 \\
0.92 \\
0.92 \\
0.93 \\
0.93 \\
0.93 \\
0.94 \\
0.94 \\
0.94 \\
0.95 \\
0.95 \\
0.95 \\
0.96 \\
0.96 \\
0.96 \\
0.97 \\
0.97 \\
0.97 \\
0.98 \\
0.98 \\
0.98 \\
0.98 \\
0.99 \\
0.99 \\
0.99 \\
0.99 \\
1.00 \\
1.00 \\
0.00\end{array}$ & $\begin{array}{l}0.00 \\
0.00 \\
0.02 \\
0.02 \\
0.02 \\
0.03 \\
0.04 \\
0.04 \\
0.05 \\
0.06 \\
0.07 \\
0.08 \\
0.09 \\
0.10 \\
0.21 \\
0.49 \\
0.56 \\
0.63 \\
0.68 \\
0.73 \\
0.77 \\
0.80 \\
0.82 \\
0.84 \\
0.85 \\
0.87 \\
0.88 \\
0.89 \\
0.90 \\
0.90 \\
0.91 \\
0.92 \\
0.92 \\
0.93 \\
0.93 \\
0.94 \\
0.94 \\
0.95 \\
0.95 \\
0.96 \\
0.96 \\
0.97 \\
0.97 \\
0.97 \\
0.98 \\
0.98 \\
0.99 \\
0.99 \\
0.99 \\
1.00\end{array}$ & $\begin{array}{l}0.00 \\
0.00 \\
0.00 \\
0.01 \\
0.02 \\
0.03 \\
0.03 \\
0.04 \\
0.04 \\
0.05 \\
0.06 \\
0.07 \\
0.07 \\
0.08 \\
0.09 \\
0.10 \\
0.12 \\
0.13 \\
0.24 \\
0.30 \\
0.45 \\
0.32 \\
0.59 \\
0.65 \\
0.71 \\
0.75 \\
0.79 \\
0.81 \\
0.84 \\
0.85 \\
0.87 \\
0.88 \\
0.89 \\
0.90 \\
0.91 \\
0.92 \\
0.92 \\
0.93 \\
0.94 \\
0.94 \\
0.95 \\
0.95 \\
0.96 \\
0.96 \\
0.97 \\
0.98 \\
0.98 \\
0.99 \\
0.99 \\
1.00\end{array}$ & $\begin{array}{l}0.00 \\
0.00 \\
0.00 \\
0.01 \\
0.02 \\
0.02 \\
0.03 \\
0.03 \\
0.04 \\
0.05 \\
0.05 \\
0.06 \\
0.07 \\
0.08 \\
0.08 \\
0.09 \\
0.10 \\
0.11 \\
0.13 \\
0.24 \\
0.15 \\
0.17 \\
0.37 \\
0.43 \\
0.49 \\
0.56 \\
0.63 \\
0.69 \\
0.75 \\
0.78 \\
0.81 \\
0.83 \\
0.85 \\
0.87 \\
0.88 \\
0.89 \\
0.90 \\
0.91 \\
0.92 \\
0.93 \\
0.94 \\
0.94 \\
0.95 \\
0.96 \\
0.96 \\
0.97 \\
0.98 \\
0.98 \\
0.99 \\
0.99\end{array}$ & $\begin{array}{l}0.00 \\
0.00 \\
0.00 \\
0.01 \\
0.02 \\
0.02 \\
0.03 \\
0.03 \\
0.04 \\
0.04 \\
0.05 \\
0.06 \\
0.06 \\
0.07 \\
0.08 \\
0.09 \\
0.09 \\
0.10 \\
0.22 \\
0.23 \\
0.24 \\
0.15 \\
0.27 \\
0.19 \\
0.36 \\
0.41 \\
0.47 \\
0.54 \\
0.61 \\
0.68 \\
0.74 \\
0.78 \\
0.82 \\
0.83 \\
0.85 \\
0.87 \\
0.88 \\
0.90 \\
0.92 \\
0.92 \\
0.93 \\
0.93 \\
0.94 \\
0.95 \\
0.96 \\
0.96 \\
0.97 \\
0.98 \\
0.99 \\
0.99\end{array}$ & $\begin{array}{l}0.00 \\
0.00 \\
0.00 \\
0.01 \\
0.02 \\
0.02 \\
0.03 \\
0.03 \\
0.04 \\
0.04 \\
0.05 \\
0.05 \\
0.06 \\
0.07 \\
0.07 \\
0.08 \\
0.09 \\
0.20 \\
0.21 \\
0.22 \\
0.23 \\
0.14 \\
0.26 \\
0.17 \\
0.29 \\
0.21 \\
0.37 \\
0.42 \\
0.49 \\
0.56 \\
0.64 \\
0.71 \\
0.76 \\
0.80 \\
0.82 \\
0.85 \\
0.86 \\
0.88 \\
0.89 \\
0.90 \\
0.91 \\
0.92 \\
0.93 \\
0.94 \\
0.93 \\
0.96 \\
0.97 \\
0.98 \\
0.98 \\
0.99\end{array}$ \\
\hline
\end{tabular}


Table 3.3.2-2d $\quad\left(p_{1}=0.05\right.$ atm)

Discharge Vold Fraction ve. Inlet Vold Fruction for varlous Homologous Flow Parameters at $\mathrm{PI}=0.05$ atm AC Motor Case

\begin{tabular}{|c|c|c|c|c|c|c|}
\hline \multirow{2}{*}{$\begin{array}{l}\text { Inlet } \\
\text { vold }\end{array}$} & \multicolumn{6}{|c|}{ Homologous Flow Paramete } \\
\hline & 0.4 & 0.6 & 0.8 & 1.0 & 2.2 & 2.4 \\
\hline $\begin{array}{l}0.00 \\
0.02 \\
0.04 \\
0.08 \\
0.08 \\
0.30 \\
0.22 \\
0.24 \\
0.26 \\
0.18 \\
0.20 \\
0.22 \\
0.24 \\
0.26 \\
0.28 \\
0.30 \\
0.32 \\
0.34 \\
0.34 \\
0.38 \\
0.40 \\
0.42\end{array}$ & $\begin{array}{l}0.00 \\
0.00 \\
0.00 \\
0.00 \\
0.00 \\
0.00 \\
0.00 \\
0.00 \\
0.00 \\
0.00 \\
0.00 \\
0.00 \\
0.00 \\
0.00 \\
0.00 \\
0.00 \\
0.83 \\
0.86 \\
0.87 \\
0.88 \\
0.89 \\
0.90 \\
0.92 \\
0.92 \\
0.92 \\
0.92 \\
0.92 \\
0.93 \\
0.94 \\
0.94 \\
0.94 \\
0.95 \\
0.95 \\
0.95 \\
0.96 \\
0.96 \\
0.96 \\
0.97 \\
0.97 \\
0.97 \\
0.98 \\
0.98 \\
0.98 \\
0.98 \\
0.99 \\
0.99 \\
0.99 \\
0.99 \\
1.00 \\
1.00 \\
\end{array}$ & $\begin{array}{l}0.00 \\
0.00 \\
0.00 \\
0.00 \\
0.00 \\
0.00 \\
0.00 \\
0.00 \\
0.00 \\
0.00 \\
0.00 \\
0.00 \\
0.00 \\
0.00 \\
0.00 \\
0.00 \\
0.00 \\
0.00 \\
0.00 \\
0.00 \\
0.00 \\
0.00 \\
0.05 \\
0.74 \\
0.78 \\
0.82 \\
0.84 \\
0.86 \\
0.88 \\
0.89 \\
0.90 \\
0.91 \\
0.92 \\
0.93 \\
0.83 \\
0.94 \\
0.94 \\
0.93 \\
0.95 \\
0.96 \\
0.96 \\
0.97 \\
0.97 \\
0.97 \\
0.98 \\
0.98 \\
0.99 \\
0.99 \\
0.99 \\
1.00\end{array}$ & $\begin{array}{l}0.00 \\
0.00 \\
0.00 \\
0.00 \\
0.00 \\
0.00 \\
0.00 \\
0.00 \\
0.00 \\
0.00 \\
0.00 \\
0.00 \\
0.00 \\
0.00 \\
0.00 \\
0.00 \\
0.00 \\
0.00 \\
0.00 \\
0.00 \\
0.00 \\
0.00 \\
0.00 \\
0.00 \\
0.00 \\
0.00 \\
0.06 \\
0.06 \\
0.66 \\
0.74 \\
0.00 \\
0.84 \\
0.86 \\
0.88 \\
0.89 \\
0.92 \\
0.92 \\
0.93 \\
0.91 \\
0.91 \\
0.95 \\
0.95 \\
0.96 \\
0.96 \\
0.97 \\
0.98 \\
0.98 \\
0.99 \\
0.99 \\
1.00\end{array}$ & $\begin{array}{l}0.00 \\
0.00 \\
0.00 \\
0.00 \\
0.00 \\
0.00 \\
0.00 \\
0.00 \\
0.00 \\
0.00 \\
0.00 \\
0.00 \\
0.00 \\
0.00 \\
0.00 \\
0.00 \\
0.00 \\
0.00 \\
0.00 \\
0.00 \\
0.00 \\
0.00 \\
0.00 \\
0.00 \\
0.00 \\
0.00 \\
0.00 \\
0.00 \\
0.05 \\
0.06 \\
0.07 \\
0.85 \\
0.69 \\
0.78 \\
0.83 \\
0.86 \\
0.88 \\
0.90 \\
0.91 \\
0.93 \\
0.93 \\
0.91 \\
0.95 \\
0.96 \\
0.96 \\
0.97 \\
0.98 \\
0.98 \\
0.99 \\
0.99 \\
0.90\end{array}$ & $\begin{array}{l}0.00 \\
0.00 \\
0.00 \\
0.00 \\
0.00 \\
0.00 \\
0.00 \\
0.00 \\
0.00 \\
0.00 \\
0.00 \\
0.00 \\
0.00 \\
0.00 \\
0.00 \\
0.00 \\
0.00 \\
0.00 \\
0.00 \\
0.00 \\
0.00 \\
0.00 \\
0.00 \\
0.00 \\
0.00 \\
0.00 \\
0.00 \\
0.00 \\
0.00 \\
0.05 \\
0.06 \\
0.07 \\
0.08 \\
0.09 \\
0.64 \\
0.77 \\
0.83 \\
0.87 \\
0.89 \\
0.00 \\
0.92 \\
0.93 \\
0.91 \\
0.95 \\
0.96 \\
0.96 \\
0.97 \\
0.98 \\
0.99 \\
0.99\end{array}$ & $\begin{array}{l}0.00 \\
0.00 \\
0.00 \\
0.00 \\
0.00 \\
0.00 \\
0.00 \\
0.00 \\
0.00 \\
0.00 \\
0.00 \\
0.00 \\
0.00 \\
0.00 \\
0.00 \\
0.00 \\
0.00 \\
0.00 \\
0.00 \\
0.00 \\
0.00 \\
0.00 \\
0.00 \\
0.00 \\
0.00 \\
0.00 \\
0.00 \\
0.00 \\
0.00 \\
0.00 \\
0.05 \\
0.06 \\
0.06 \\
0.07 \\
0.09 \\
0.41 \\
0.70 \\
0.82 \\
0.85 \\
0.88 \\
0.90 \\
0.92 \\
0.03 \\
0.94 \\
0.95 \\
0.96 \\
0.97 \\
0.98 \\
0.98 \\
0.99\end{array}$ \\
\hline
\end{tabular}


Table 3.3.2-3n $\quad\left(p_{1}=3 \mathrm{~atm}\right)$

statlo preseure Rise in pola ve. Inlet vold rraction for Varlous Honologou Flow Parameters at $P_{2}$ - $J$ at Ac Motor cane

\begin{tabular}{|c|c|c|c|c|c|c|}
\hline \multirow{2}{*}{$\begin{array}{l}\text { Inlet } \\
\text { vold } \\
\text { eraetion }\end{array}$} & \multicolumn{6}{|c|}{ Homologous Flow paramoter } \\
\hline & 0.4 & 0.6 & 0.8 & 2.0 & 2.2 & 2.4 \\
\hline 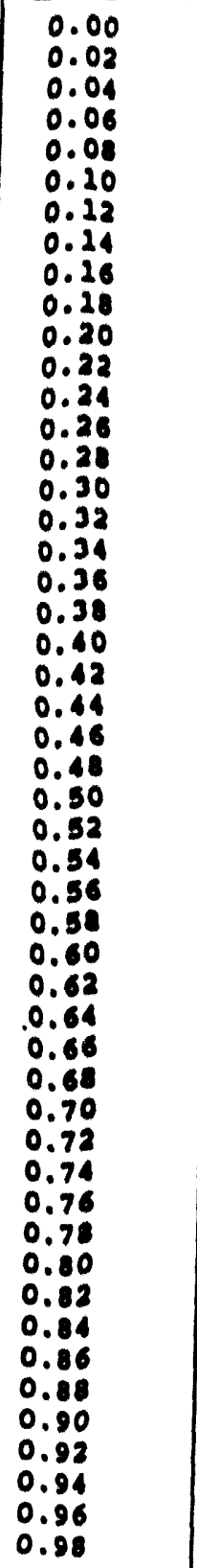 &  &  & 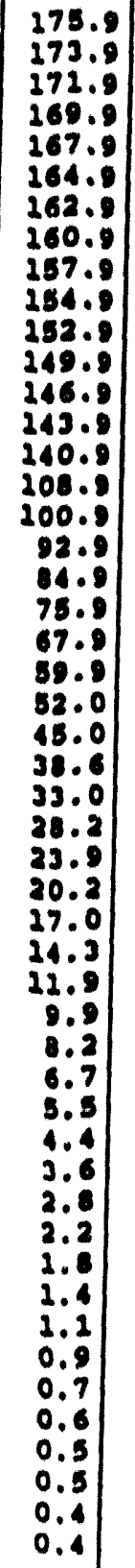 & 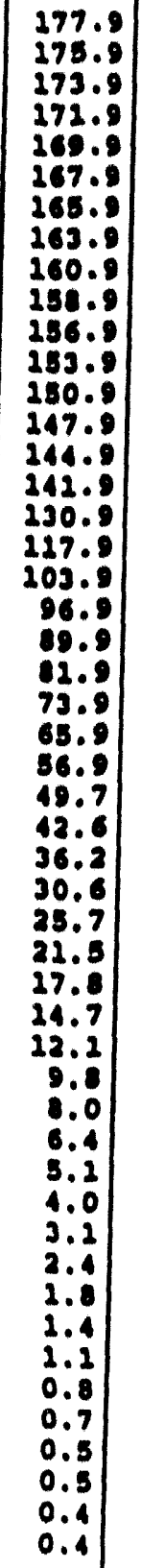 & 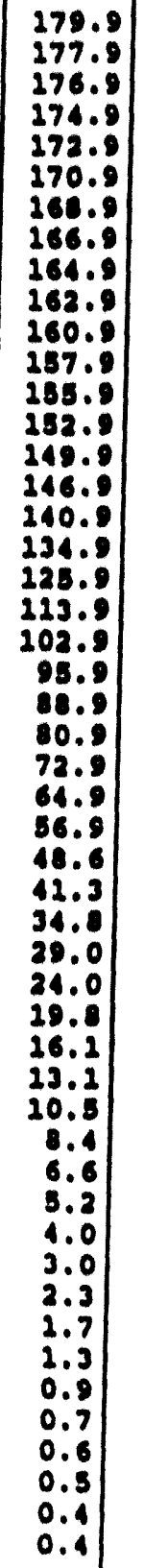 & 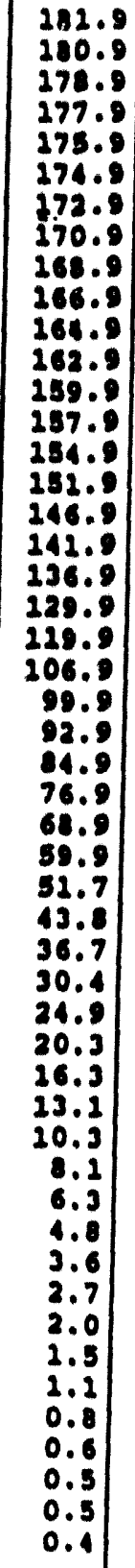 \\
\hline
\end{tabular}




\begin{tabular}{|c|c|}
\hline 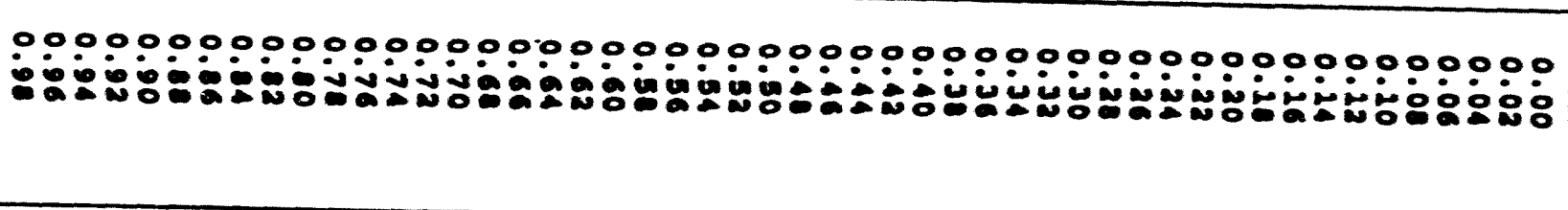 & \\
\hline 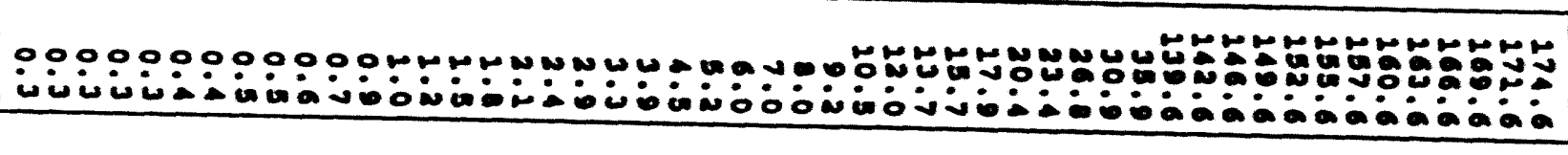 & : \\
\hline 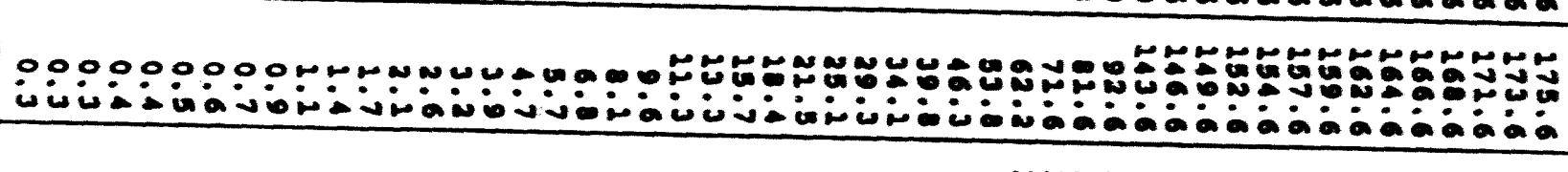 & \begin{tabular}{|l|} 
\\
$\vdots$ \\
\end{tabular} \\
\hline  & 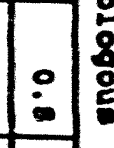 \\
\hline  & 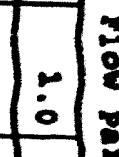 \\
\hline Oo & 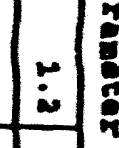 \\
\hline 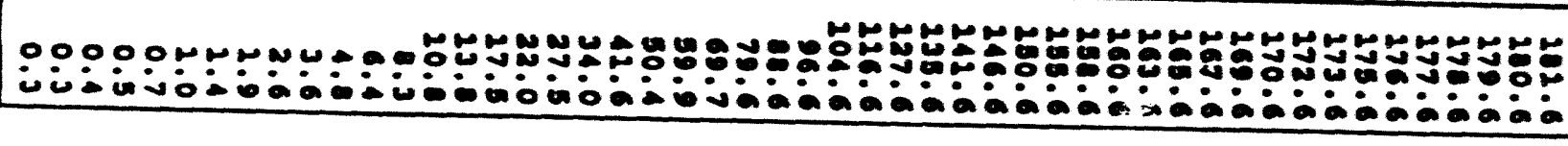 & מל: \\
\hline
\end{tabular}


Table 3.3.2-3c $\quad\left(p_{1}=1 \mathrm{~atm}\right)$

statele preseure Rlee in pela ve. Indet vold Fraction for Varlous Howologous flow paraneters at $P_{1}$ i atm Ac Motor case

\begin{tabular}{|c|c|c|c|c|c|c|}
\hline \multirow{2}{*}{$\begin{array}{l}\text { Indet } \\
\text { vold } \\
\text { eraotion }\end{array}$} & \multicolumn{6}{|c|}{ Honologoue Flow Parameter } \\
\hline & 0.4 & 0.6 & 0.8 & 2.0 & 2.2 & 2. \\
\hline $\begin{array}{l}0.00 \\
0.02 \\
0.04 \\
0.04 \\
0.04 \\
0.10 \\
0.12\end{array}$ & 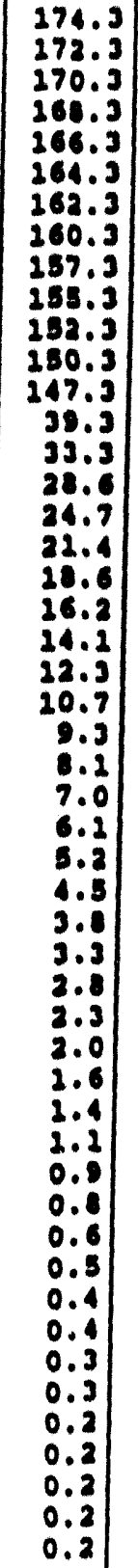 & 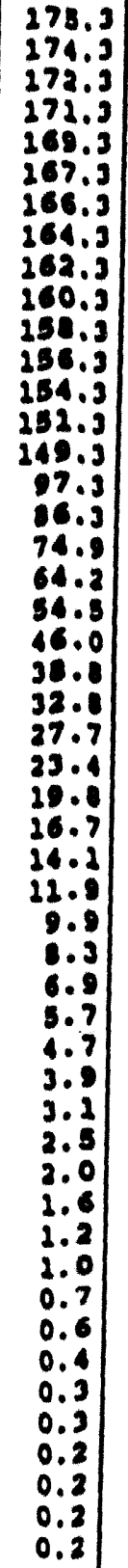 & 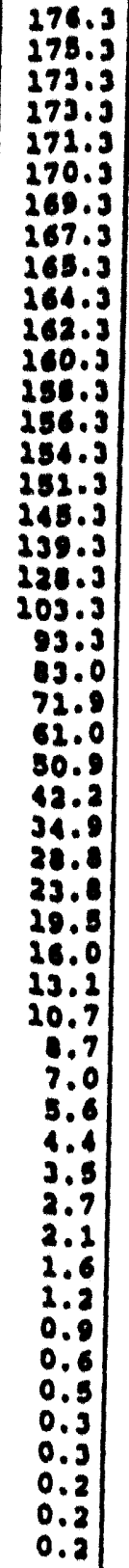 & 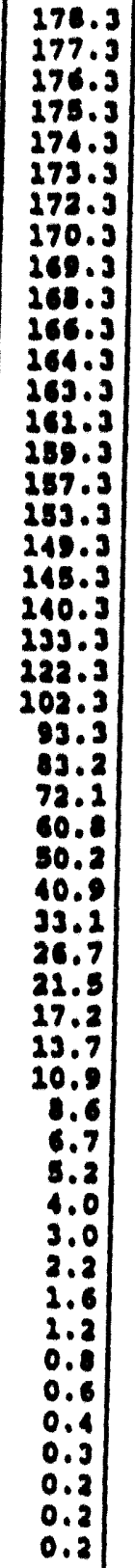 & 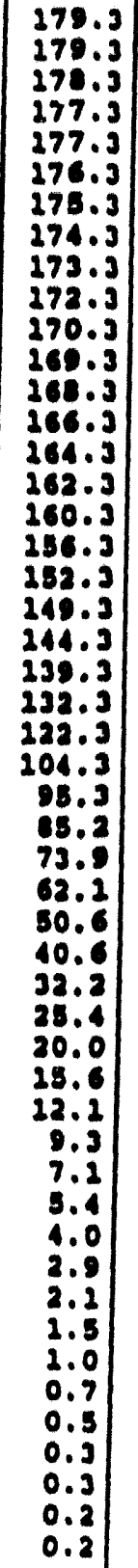 & 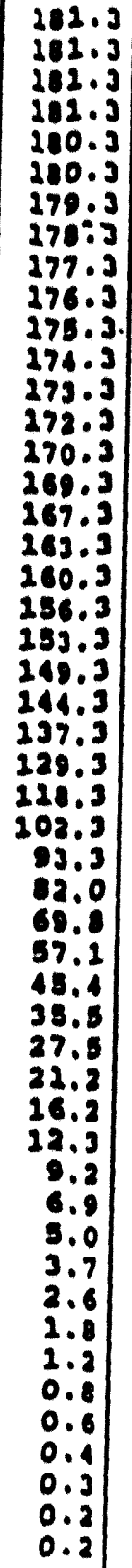 \\
\hline
\end{tabular}


Table 3.3.2-3d $\quad\left(p_{1}=0.05 \mathrm{~atm}\right)$

statle proseure Rise in pala va. Indet Vold rraction cor varlous Howologoue Flow Paramotere at P1 $=.05$ atw AC Motor Care

\begin{tabular}{|c|c|c|c|c|c|c|}
\hline \multirow{2}{*}{$\begin{array}{l}\text { Indet } \\
\text { vold } \\
\text { exaetion }\end{array}$} & \multicolumn{6}{|c|}{ Honologous Flow Paraneter } \\
\hline & 0.4 & 0.6 & 0.8 & 2.0 & 2.2 & 2.4 \\
\hline 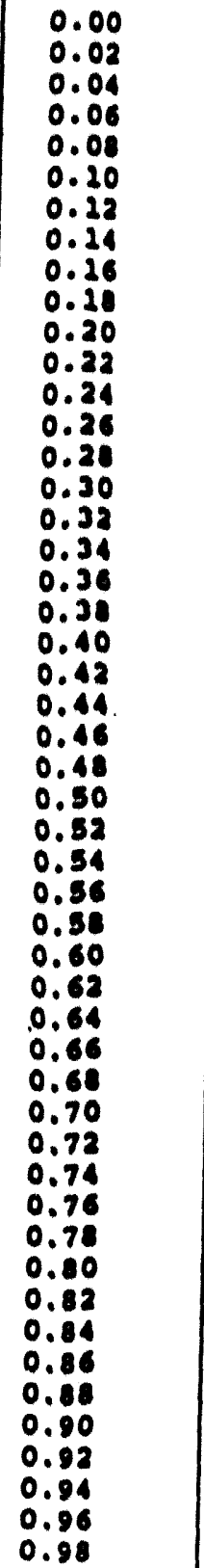 & 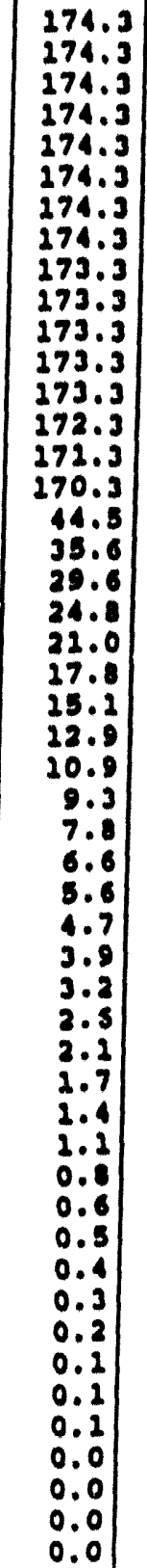 & 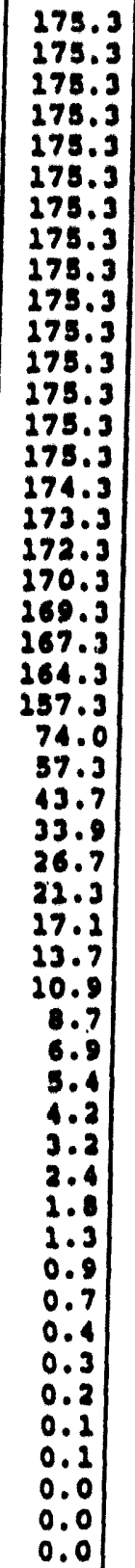 & 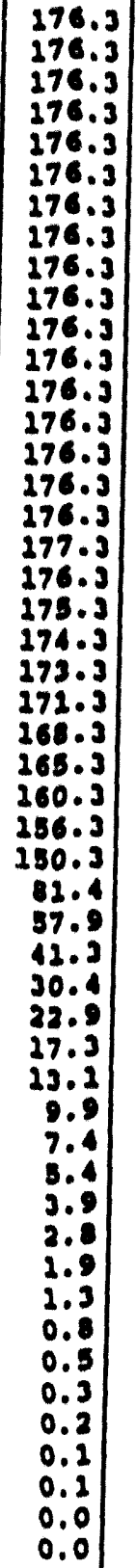 &  & 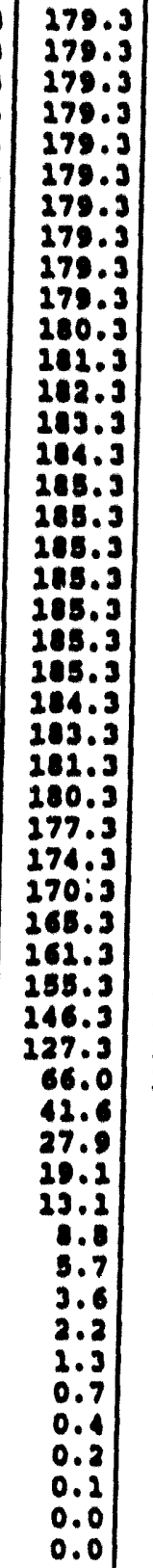 & 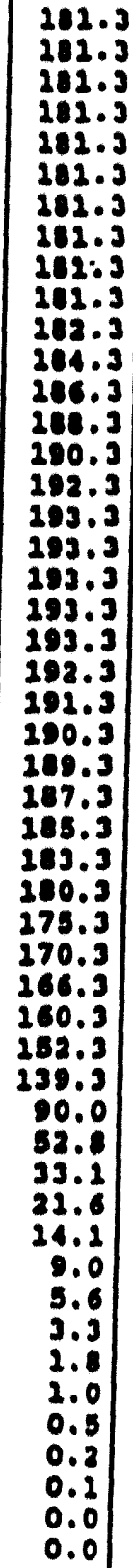 \\
\hline
\end{tabular}


Table 3.3.2-4a $\quad\left(p_{1}=3 \mathrm{~atm}\right)$

Normalised Relative Veloolty of Water at Vane Ixit ve. Inlet Vold Fraction gor Varlous Homologous Flow Parametera at PI i j ate AC Notor Case

\begin{tabular}{|c|c|c|c|c|c|c|}
\hline \multirow{2}{*}{$\begin{array}{l}\text { Indet } \\
\text { vold } \\
\text { Praetion }\end{array}$} & \multicolumn{6}{|c|}{ Honologoue Flow paraneter } \\
\hline & 0.1 & 0.6 & 0.1 & 1.0 & 2.2 & 2.4 \\
\hline $\begin{array}{l}0.00 \\
0.02 \\
0.04 \\
0.06 \\
0.03 \\
0.20 \\
0.212 \\
0.14 \\
0.26 \\
0.18 \\
0.20 \\
0.22 \\
0.24 \\
0.26 \\
0.28 \\
0.30 \\
0.32 \\
0.34 \\
0.34 \\
0.38 \\
0.10 \\
0.42\end{array}$ & 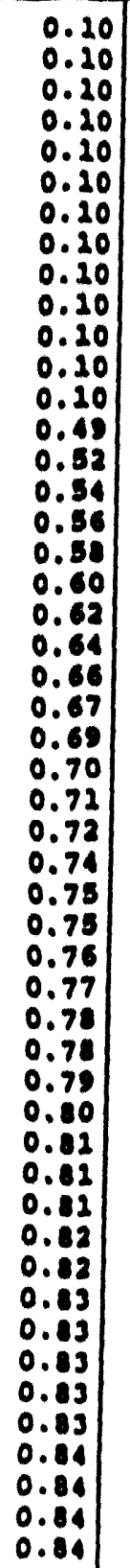 & 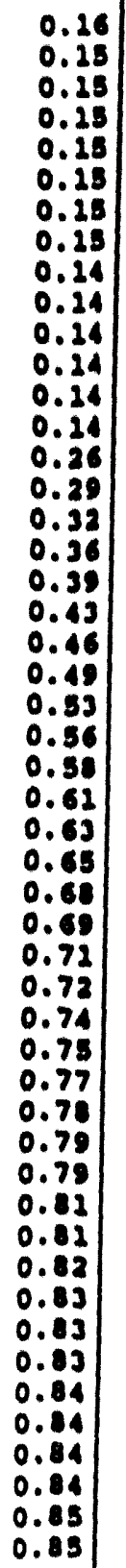 & 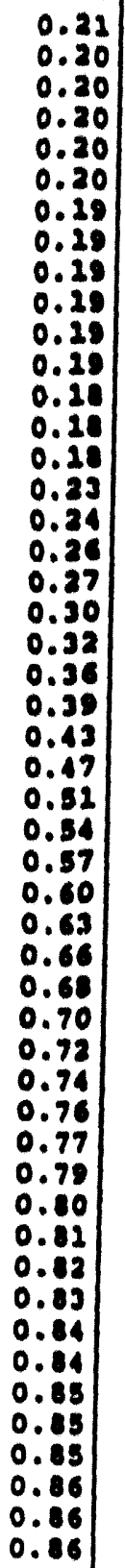 & 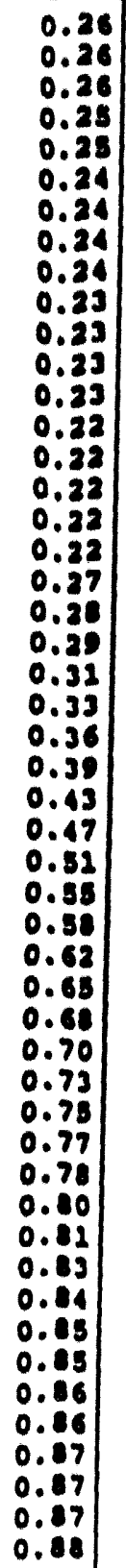 & 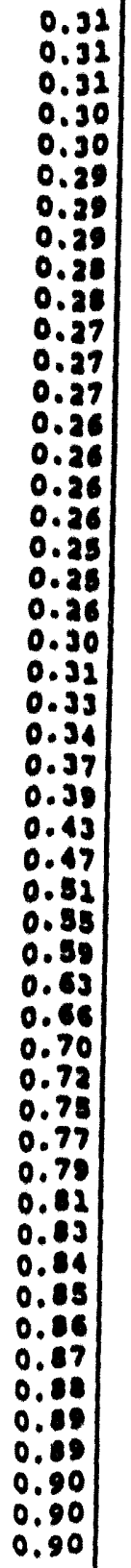 &  \\
\hline
\end{tabular}


Table 3.3.2-4b

$\left(p_{1}=2 \mathrm{~atm}\right)$

Normalized Relative Velocity of water at Vane Exit vs. Inlet vold Fraction lor Various Homologous Flow Parameters at $P 1=2$ atw AC Motor Case

\begin{tabular}{|c|c|c|c|c|c|c|}
\hline \multirow{2}{*}{$\begin{array}{l}\text { Inlet } \\
\text { Vold } \\
\text { Fraction }\end{array}$} & \multicolumn{6}{|c|}{ Homologous Flow Parameter } \\
\hline & 0.4 & 0.6 & 0.8 & 1.0 & 1.2 & 1.4 \\
\hline $\begin{array}{l}0.00 \\
0.02 \\
0.04 \\
0.04 \\
0.06 \\
0.08 \\
0.10 \\
0.12\end{array}$ & 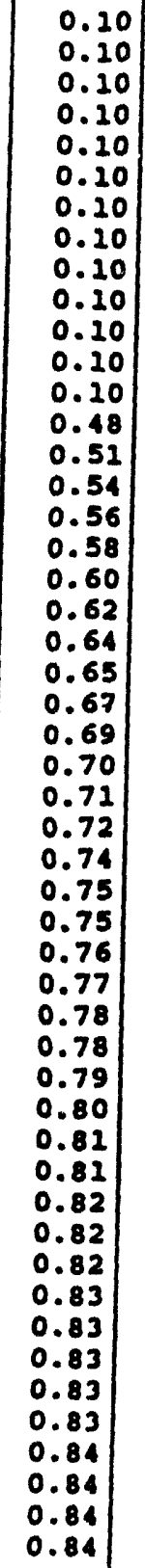 & $\begin{array}{l}0.16 \\
0.15 \\
0.15 \\
0.15 \\
0.15 \\
0.15 \\
0.25 \\
0.14 \\
0.14 \\
0.14 \\
0.24 \\
0.14 \\
0.14 \\
0.13 \\
0.24 \\
0.26 \\
0.30 \\
0.33 \\
0.37 \\
0.41 \\
0.44 \\
0.48 \\
0.51 \\
0.54 \\
0.57 \\
0.60 \\
0.62 \\
0.65 \\
0.67 \\
0.69 \\
0.71 \\
0.72 \\
0.74 \\
0.75 \\
0.76 \\
0.78 \\
0.78 \\
0.79 \\
0.81 \\
0.81 \\
0.82 \\
0.83 \\
0.83 \\
0.83 \\
0.84 \\
0.84 \\
0.84 \\
0.85 \\
0.85 \\
0.85\end{array}$ & $\begin{array}{l}0.21 \\
0.20 \\
0.20 \\
0.20 \\
0.20 \\
0.19 \\
0.19 \\
0.19 \\
0.19 \\
0.19 \\
0.18 \\
0.18 \\
0.18 \\
0.18 \\
0.17 \\
0.17 \\
0.22 \\
0.23 \\
0.24 \\
0.26 \\
0.29 \\
0.32 \\
0.36 \\
0.40 \\
0.44 \\
0.18 \\
0.52 \\
0.56 \\
0.59 \\
0.62 \\
0.65 \\
0.67 \\
0.70 \\
0.72 \\
0.74 \\
0.76 \\
0.77 \\
0.79 \\
0.80 \\
0.81 \\
0.82 \\
0.83 \\
0.84 \\
0.84 \\
0.85 \\
0.85 \\
0.85 \\
0.86 \\
0.86 \\
0.86\end{array}$ & $\begin{array}{l}0.26 \\
0.26 \\
0.25 \\
0.25 \\
0.25 \\
0.24 \\
0.24 \\
0.23 \\
0.23 \\
0.23 \\
0.23 \\
0.22 \\
0.22 \\
0.22 \\
0.21 \\
0.21 \\
0.22 \\
0.21 \\
0.22 \\
0.25 \\
0.26 \\
0.27 \\
0.29 \\
0.32 \\
0.35 \\
0.39 \\
0.43 \\
0.48 \\
0.52 \\
0.56 \\
0.60 \\
0.64 \\
0.67 \\
0.70 \\
0.72 \\
0.75 \\
0.77 \\
0.78 \\
0.80 \\
0.82 \\
0.83 \\
0.84 \\
0.85 \\
0.85 \\
0.86 \\
0.87 \\
0.87 \\
0.87 \\
0.87 \\
0.88\end{array}$ & $\begin{array}{l}0.31 \\
0.31 \\
0.30 \\
0.30 \\
0.30 \\
0.29 \\
0.29 \\
0.28 \\
0.28 \\
0.27 \\
0.27 \\
0.27 \\
0.26 \\
0.26 \\
0.25 \\
0.25 \\
0.25 \\
0.24 \\
0.24 \\
0.24 \\
0.24 \\
0.28 \\
0.29 \\
0.30 \\
0.32 \\
0.34 \\
0.38 \\
0.42 \\
0.47 \\
0.52 \\
0.56 \\
0.60 \\
0.64 \\
0.68 \\
0.71 \\
0.74 \\
0.77 \\
0.79 \\
0.81 \\
0.83 \\
0.84 \\
0.85 \\
0.86 \\
0.87 \\
0.88 \\
0.89 \\
0.89 \\
0.90 \\
0.90 \\
0.90\end{array}$ & $\begin{array}{l}0.37 \\
0.36 \\
0.35 \\
0.35 \\
0.35 \\
0.34 \\
0.33 \\
0.33 \\
0.32 \\
0.32 \\
0.31 \\
0.31 \\
0.30 \\
0.30 \\
0.30 \\
0.29 \\
0.29 \\
0.28 \\
0.28 \\
0.28 \\
0.27 \\
0.27 \\
0.27 \\
0.32 \\
0.32 \\
0.34 \\
0.36 \\
0.39 \\
0.43 \\
0.48 \\
0.53 \\
0.58 \\
0.63 \\
0.67 \\
0.71 \\
0.74 \\
0.77 \\
0.80 \\
0.82 \\
0.84 \\
0.86 \\
0.87 \\
0.88 \\
0.89 \\
0.90 \\
0.91 \\
0.91 \\
0.92 \\
0.93 \\
0.93\end{array}$ \\
\hline
\end{tabular}


Table 3.3.2-4c $\quad\left(p_{1}=1 \mathrm{~atm}\right)$

Normalized Relative Velocity of Water at Vane Exit vs. Inlet Void

Fraction for Various Homologous Flow Parameters at PI = 1 atm AC Motor Case

\begin{tabular}{|c|c|c|c|c|c|c|}
\hline \multirow{2}{*}{$\begin{array}{l}\text { Inlet } \\
\text { Vold } \\
\text { Fraction }\end{array}$} & \multicolumn{6}{|c|}{ Homologous Flow parameter } \\
\hline & 0.4 & 0.6 & 0.8 & 2.0 & 1.2 & 2.4 \\
\hline $\begin{array}{l}0.00 \\
0.02 \\
0.04 \\
0.04 \\
0.06 \\
0.08 \\
0.10 \\
0.12 \\
0.24 \\
0.16 \\
0.18 \\
0.20 \\
0.22 \\
0.24 \\
0.26 \\
0.28 \\
0.30 \\
0.32 \\
0.34 \\
0.36 \\
0.38 \\
0.40 \\
0.42\end{array}$ &  & $\begin{array}{l}0.16 \\
0.25 \\
0.15 \\
0.15 \\
0.15 \\
0.14 \\
0.14 \\
0.14 \\
0.24 \\
0.14 \\
0.13 \\
0.13 \\
0.13 \\
0.13 \\
0.13 \\
0.22 \\
0.24 \\
0.28 \\
0.32 \\
0.36 \\
0.40 \\
0.44 \\
0.48 \\
0.52 \\
0.55 \\
0.58 \\
0.61 \\
0.64 \\
0.66 \\
0.68 \\
0.70 \\
0.72 \\
0.73 \\
0.75 \\
0.76 \\
0.77 \\
0.78 \\
0.79 \\
0.81 \\
0.81 \\
0.82 \\
0.83 \\
0.83 \\
0.83 \\
0.84 \\
0.84 \\
0.84 \\
0.85 \\
0.85 \\
0.85\end{array}$ & 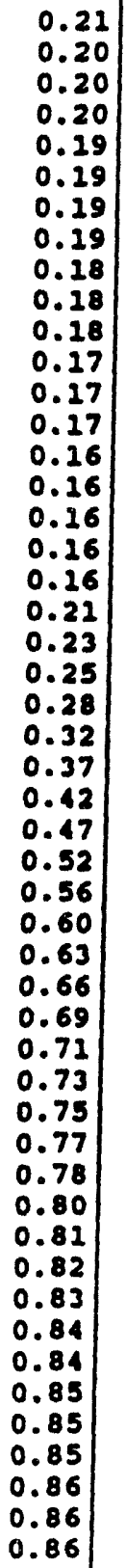 & 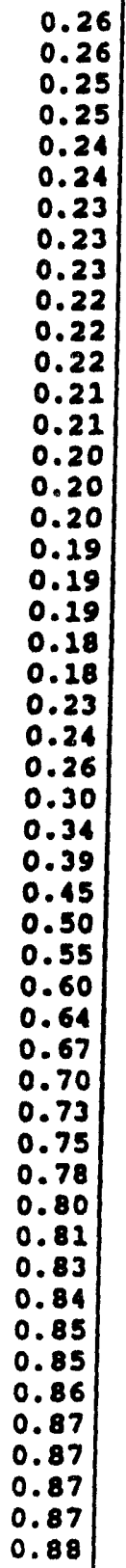 & $\begin{array}{l}0.31 \\
0.32 \\
0.30 \\
0.30 \\
0.29 \\
0.29 \\
0.28 \\
0.28 \\
0.27 \\
0.27 \\
0.26 \\
0.26 \\
0.25 \\
0.25 \\
0.24 \\
0.24 \\
0.24 \\
0.24 \\
0.23 \\
0.23 \\
0.22 \\
0.22 \\
0.21 \\
0.21 \\
0.21 \\
0.21 \\
0.25 \\
0.26 \\
0.28 \\
0.28 \\
0.31 \\
0.35 \\
0.41 \\
0.48\end{array}$ & $\begin{array}{l}0.37 \\
0.36 \\
0.35 \\
0.35 \\
0.34 \\
0.34 \\
0.33 \\
0.32 \\
0.32 \\
0.31 \\
0.30 \\
0.30 \\
0.30 \\
0.29 \\
0.28 \\
0.28 \\
0.27 \\
0.27 \\
0.26 \\
0.26 \\
0.25 \\
0.25 \\
0.24 \\
0.24 \\
0.23 \\
0.23 \\
0.28 \\
0.29 \\
0.31 \\
0.35 \\
0.41 \\
0.48 \\
0.51 \\
0.61 \\
0.66 \\
0.71 \\
0.75 \\
0.78 \\
0.81 \\
0.83 \\
0.85 \\
0.87 \\
0.88 \\
0.89 \\
0.90 \\
0.91 \\
0.91 \\
0.92 \\
0.93 \\
0.93\end{array}$ \\
\hline
\end{tabular}


Table 3.3.2-4d $\quad\left(p_{1}=0.05 \mathrm{~atm}\right)$

Normalized Relative Velocity of Water at Vane Exit vs. Inlet Void Fraction for Various Homologous Flow Parameters at P1 $=0.05$ atm AC Motor Case

\begin{tabular}{|c|c|c|c|c|c|c|}
\hline \multirow{2}{*}{$\begin{array}{l}\text { Inlet } \\
\text { Void } \\
\text { Fraction }\end{array}$} & \multicolumn{6}{|c|}{ Homologous Flow Parameter } \\
\hline & 0.4 & 0.6 & 0.8 & 2.0 & 1.2 & 2.4 \\
\hline 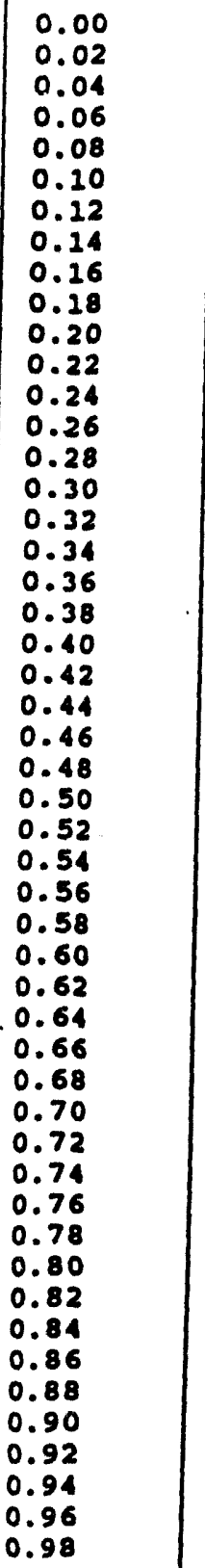 & 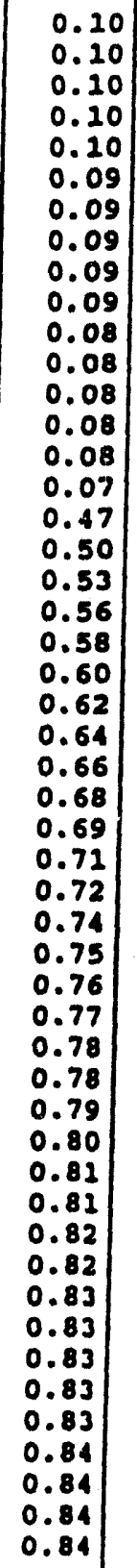 & $\begin{array}{l}0.16 \\
0.15 \\
0.15 \\
0.15 \\
0.14 \\
0.14 \\
0.14 \\
0.13 \\
0.13 \\
0.13 \\
0.13 \\
0.12 \\
0.12 \\
0.12 \\
0.11 \\
0.12 \\
0.11 \\
0.10 \\
0.10 \\
0.10 \\
0.10 \\
0.09 \\
0.09 \\
0.09 \\
0.33 \\
0.38 \\
0.43 \\
0.48 \\
0.52 \\
0.52 \\
0.56 \\
0.59\end{array}$ & 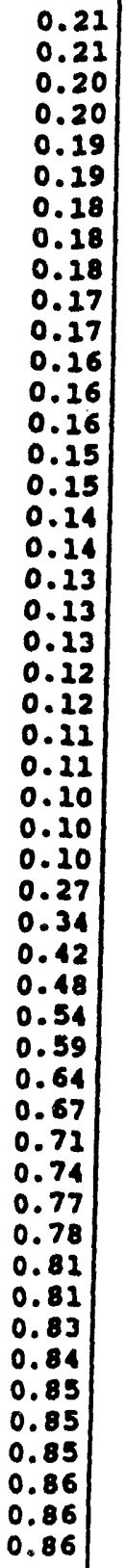 & $\begin{array}{l}0.26 \\
0.26 \\
0.25 \\
0.25 \\
0.24 \\
0.23 \\
0.23 \\
0.22 \\
0.22 \\
0.21 \\
0.21 \\
0.20 \\
0.20 \\
0.19 \\
0.19 \\
0.18 \\
0.18 \\
0.27 \\
0.17 \\
0.16 \\
0.16 \\
0.16 \\
0.15 \\
0.14 \\
0.14 \\
0.14 \\
0.13 \\
0.12 \\
0.12 \\
0.12 \\
0.12 \\
0.22 \\
0.11 \\
0.22 \\
0.31 \\
0.41 \\
0.30 \\
0.57 \\
0.63\end{array}$ & 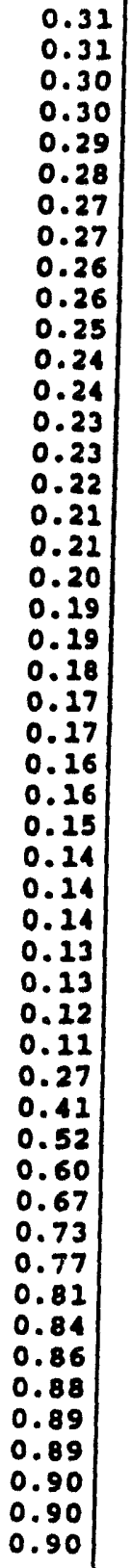 & 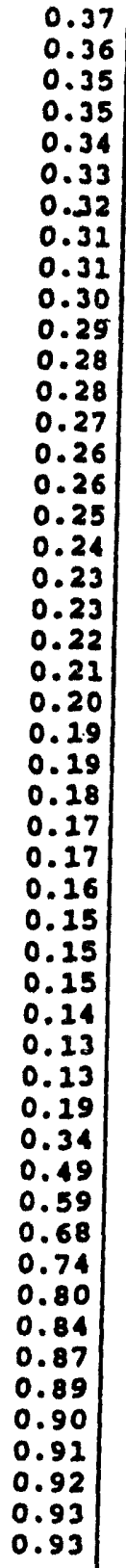 \\
\hline
\end{tabular}


Table 3.3.2-5a $\quad\left(p_{1}=3 \mathrm{~atm}\right)$

Normalized Rerative Velocity of Air at Vane Exit vs. Inlet Void
Fraction for Various Homologous Flow Parameters at PI 3 atm Fraction for Varlous Homologous Flow Parameters at P1 $=3$ atm

\begin{tabular}{|c|c|c|c|c|c|c|}
\hline \multirow{2}{*}{$\begin{array}{l}\text { Inlat } \\
\text { Vold } \\
\text { Fraction }\end{array}$} & \multicolumn{6}{|c|}{ Homologous Flow Parameter } \\
\hline & 0.4 & 0.6 & 0.8 & 1.0 & 1.2 & 1.4 \\
\hline $\begin{array}{l}0.00 \\
0.02 \\
0.04 \\
0.06 \\
0.08 \\
0.10 \\
0.12 \\
0.14 \\
0.16 \\
0.18 \\
0.20 \\
0.22 \\
0.24 \\
0.26 \\
0.28 \\
0.30 \\
0.32 \\
0.34 \\
0.36 \\
0.38 \\
0.40 \\
0.42 \\
0.44 \\
0.46 \\
0.48 \\
0.50 \\
0.52 \\
0.54 \\
0.56 \\
0.58 \\
0.60 \\
0.62 \\
0.64 \\
0.66 \\
0.68 \\
0.70 \\
0.72 \\
0.74 \\
0.76 \\
0.78 \\
0.80 \\
0.82 \\
0.84 \\
0.86 \\
0.88 \\
0.90 \\
0.922 \\
0.94 \\
0.96 \\
0.98\end{array}$ & $\begin{array}{l}N A \\
0.05 \\
0.05 \\
0.05 \\
0.05 \\
0.05 \\
0.05 \\
0.05 \\
0.05 \\
0.05 \\
0.05 \\
0.05 \\
0.05 \\
0.02 \\
0.02 \\
0.03 \\
0.03 \\
0.03 \\
0.03 \\
0.04 \\
0.04 \\
0.04 \\
0.04 \\
0.05 \\
0.05 \\
0.05 \\
0.05 \\
0.06 \\
0.06 \\
0.06 \\
0.06 \\
0.06 \\
0.07 \\
0.07 \\
0.07 \\
0.07 \\
0.08 \\
0.08 \\
0.08 \\
0.08 \\
0.08 \\
0.09 \\
0.09 \\
0.09 \\
0.09 \\
0.09 \\
0.20 \\
0.20 \\
0.10 \\
0.10\end{array}$ & $\begin{array}{l}\text { NA } \\
0.10 \\
0.10 \\
0.10 \\
0.09 \\
0.09 \\
0.09 \\
0.09 \\
0.09 \\
0.09 \\
0.09 \\
0.09 \\
0.09 \\
0.09 \\
0.03 \\
0.04 \\
0.04 \\
0.04 \\
0.04 \\
0.05 \\
0.05 \\
0.05 \\
0.06 \\
0.06 \\
0.07 \\
0.07 \\
0.07 \\
0.08 \\
0.08 \\
0.09 \\
0.09 \\
0.10 \\
0.10 \\
0.10 \\
0.11 \\
0.11 \\
0.11 \\
0.12 \\
0.12 \\
0.13 \\
0.13 \\
0.13 \\
0.13 \\
0.24 \\
0.14 \\
0.14 \\
0.14 \\
0.15 \\
0.15 \\
0.15\end{array}$ & $\begin{array}{l}\text { NA } \\
0.00 \\
0.15 \\
0.14 \\
0.24 \\
0.14 \\
0.14 \\
0.14 \\
0.14 \\
0.13 \\
0.23 \\
0.13 \\
0.23 \\
0.13 \\
0.13 \\
0.07 \\
0.07 \\
0.07 \\
0.07 \\
0.07 \\
0.07 \\
0.07 \\
0.07 \\
0.08 \\
0.08 \\
0.09 \\
0.09 \\
0.10 \\
0.10 \\
0.12 \\
0.12 \\
0.22 \\
0.23 \\
0.13 \\
0.14 \\
0.15 \\
0.15 \\
0.16 \\
0.16 \\
0.27 \\
0.17 \\
0.17 \\
0.18 \\
0.28 \\
0.19 \\
0.19 \\
0.29 \\
0.20 \\
0.20 \\
0.20\end{array}$ & $\begin{array}{l}N A \\
0.00 \\
0.20 \\
0.19 \\
0.19 \\
0.19 \\
0.29 \\
0.28 \\
0.18 \\
0.18 \\
0.18 \\
0.17 \\
0.17 \\
0.17 \\
0.17 \\
0.27 \\
0.27 \\
0.17 \\
0.10 \\
0.20 \\
0.20 \\
0.20 \\
0.10 \\
0.10 \\
0.10 \\
0.12 \\
0.11 \\
0.12 \\
0.12 \\
0.13 \\
0.14 \\
0.15 \\
0.16 \\
0.17 \\
0.27 \\
0.16 \\
0.29 \\
0.19 \\
0.20 \\
0.21 \\
0.21 \\
0.22 \\
0.23 \\
0.23 \\
0.23 \\
0.24 \\
0.24 \\
0.25 \\
0.25 \\
0.26\end{array}$ & $\begin{array}{l}\text { MA } \\
0.00 \\
0.25 \\
0.24 \\
0.24 \\
0.24 \\
0.23 \\
0.23 \\
0.23 \\
0.22 \\
0.22 \\
0.22 \\
0.22 \\
0.21 \\
0.21 \\
0.21 \\
0.21 \\
0.20 \\
0.20 \\
0.29 \\
0.14 \\
0.14 \\
0.13 \\
0.13 \\
0.13 \\
0.14 \\
0.24 \\
0.24 \\
0.15 \\
0.26 \\
0.16 \\
0.17 \\
0.18 \\
0.18 \\
0.21 \\
0.22 \\
0.22 \\
0.23 \\
0.24 \\
0.25 \\
0.26 \\
0.26 \\
0.27 \\
0.28 \\
0.28 \\
0.29 \\
0.29 \\
0.30 \\
0.30 \\
0.31\end{array}$ & $\begin{array}{l}N A \\
0.00 \\
0.30 \\
0.29 \\
0.29 \\
0.28 \\
0.28 \\
0.28 \\
0.27 \\
0.27 \\
0.26 \\
0.26 \\
0.26 \\
0.26 \\
0.25 \\
0.25 \\
0.24 \\
0.24 \\
0.24 \\
0.24 \\
0.24 \\
0.27 \\
0.27 \\
0.27 \\
0.27 \\
0.17 \\
0.27 \\
0.17 \\
0.27 \\
0.28 \\
0.29 \\
0.20 \\
0.21 \\
0.22 \\
0.24 \\
0.25 \\
0.26 \\
0.27 \\
0.28 \\
0.29 \\
0.30 \\
0.31 \\
0.32 \\
0.32 \\
0.33 \\
0.34 \\
0.34 \\
0.35 \\
0.35 \\
0.36\end{array}$ \\
\hline
\end{tabular}


Table 3.3.2-5b $\quad\left(p_{1}=2 \mathrm{~atm}\right)$

Normalized Rerative Velocity of Air at Vane Exit ve. Inlet Vold Fraction for Various Honologous flow parametere at $P 1=2$ atm AC Motor Case

\begin{tabular}{|c|c|c|c|c|c|c|}
\hline \multirow{2}{*}{$\begin{array}{l}\text { Inlet } \\
\text { Void } \\
\text { Iraction }\end{array}$} & \multicolumn{6}{|c|}{ Honologour Flow Parameter } \\
\hline & 0.4 & 0.6 & 0.8 & 2.0 & 1.2 & 1.4 \\
\hline $\begin{array}{l}0.00 \\
0.02 \\
0.04 \\
0.06 \\
0.08 \\
0.10 \\
0.12 \\
0.14 \\
0.16 \\
0.18 \\
0.20 \\
0.22 \\
0.24 \\
0.26 \\
0.28 \\
0.30 \\
0.32 \\
0.34 \\
0.36 \\
0.38 \\
0.40 \\
0.42 \\
0.44 \\
0.46 \\
0.48 \\
0.50 \\
0.52 \\
0.54 \\
0.56 \\
0.58 \\
0.60 \\
0.62 \\
0.64 \\
0.64 \\
0.64 \\
0.70 \\
0.72\end{array}$ & $\begin{array}{l}\text { NA } \\
0.05 \\
0.05 \\
0.05 \\
0.05 \\
0.05 \\
0.05 \\
0.04 \\
0.04 \\
0.04 \\
0.04 \\
0.04 \\
0.04 \\
0.02 \\
0.02 \\
0.02 \\
0.03 \\
0.03 \\
0.03 \\
0.03 \\
0.03 \\
0.03 \\
0.04 \\
0.04 \\
0.04 \\
0.05 \\
0.05 \\
0.05 \\
0.05 \\
0.05 \\
0.06 \\
0.06 \\
0.06 \\
0.06 \\
0.06 \\
0.07 \\
0.07 \\
0.07 \\
0.07 \\
0.07 \\
0.08 \\
0.08 \\
0.00\end{array}$ & 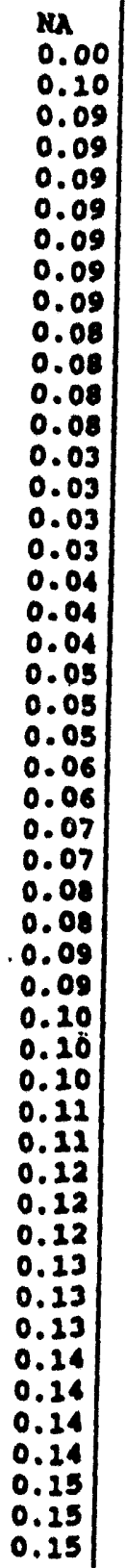 & 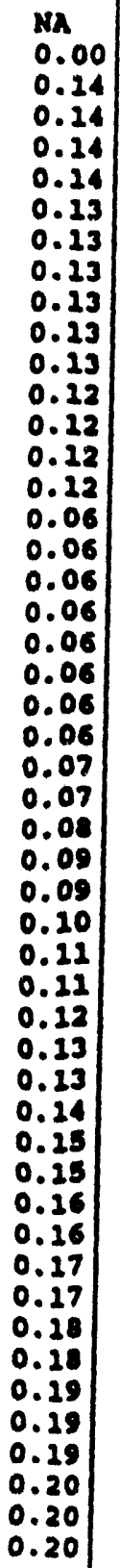 &  & 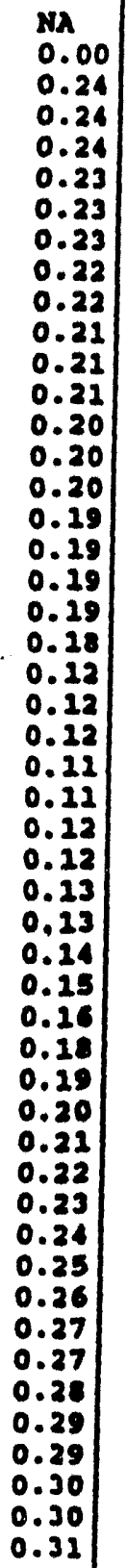 & $\begin{array}{l}N A \\
0.00 \\
0.30 \\
0.29 \\
0.28 \\
0.28 \\
0.28 \\
0.27 \\
0.27 \\
0.26 \\
0.26 \\
0.25 \\
0.25 \\
0.24 \\
0.24 \\
0.24 \\
0.23 \\
0.23 \\
0.22 \\
0.22 \\
0.22 \\
0.22 \\
0.22 \\
0.15 \\
0.15 \\
0.15 \\
0.24 \\
0.24 \\
0.15 \\
0.15 \\
0.16 \\
0.17 \\
0.18 \\
0.20 \\
0.21 \\
0.23 \\
0.24 \\
0.26 \\
0.27 \\
0.28 \\
0.29 \\
0.30 \\
0.33 \\
0.32 \\
0.33 \\
0.34 \\
0.34 \\
0.35 \\
0.35 \\
0.36\end{array}$ \\
\hline
\end{tabular}


Table 3.3.2-5c $\quad\left(p_{q}=1\right.$ atm $)$

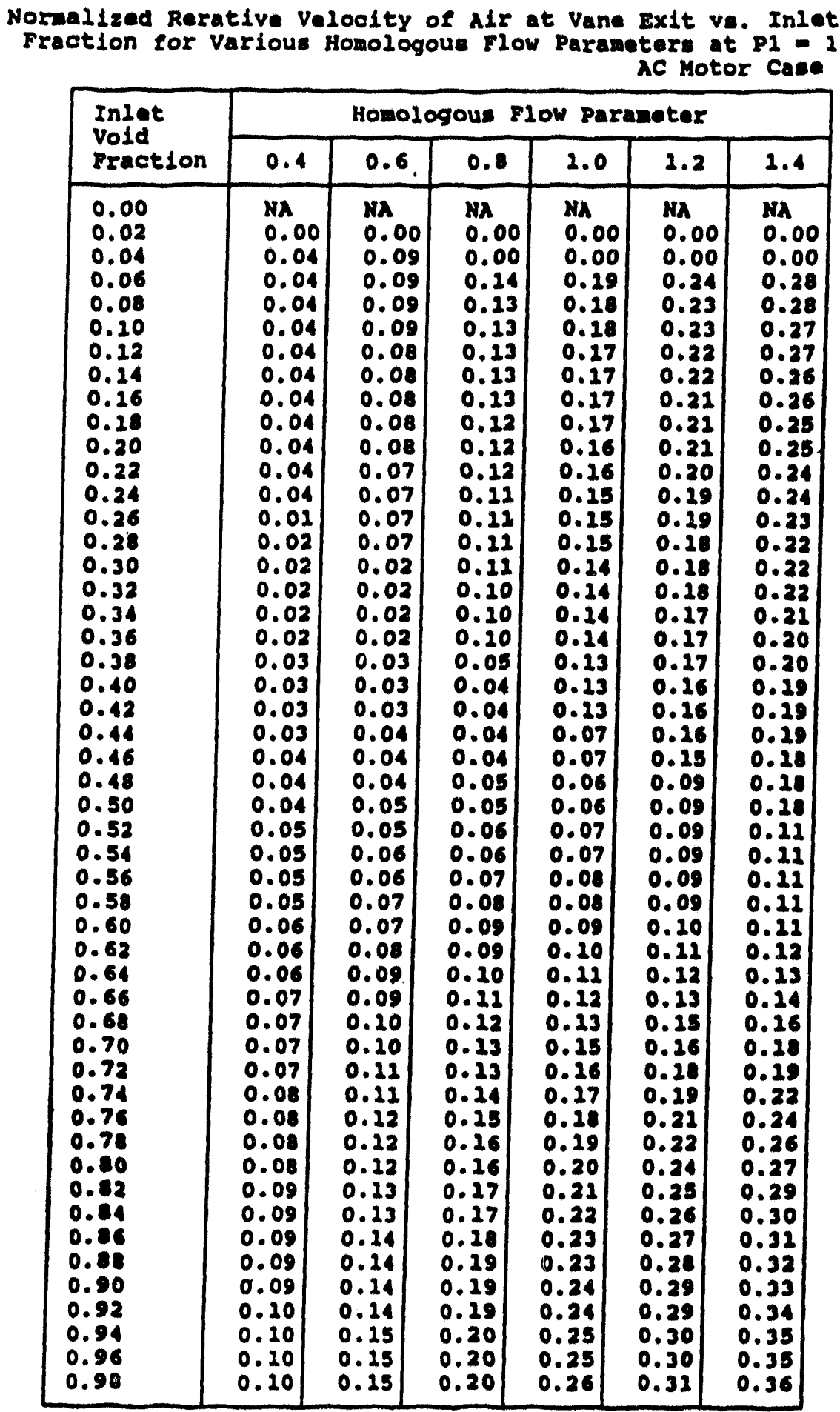


Table 3.3.2-5d $\quad\left(p_{1}=0.05 \mathrm{~atm}\right)$

Normalized Rerative Velocity of Air at Vane Exit ve. Inlet Vold Fraction for Varlous Homologous Flow Paraseters at P1 $=0.05$ atm AC Motor Case

\begin{tabular}{|c|c|c|c|c|c|c|}
\hline \multirow{2}{*}{$\begin{array}{l}\text { Inlat } \\
\text { Vold } \\
\text { Iraotion }\end{array}$} & \multicolumn{6}{|c|}{ Homologous Flow Parameter } \\
\hline & 0.4 & 0.6 & 0.8 & 2.0 & 1.2 & 2.4 \\
\hline 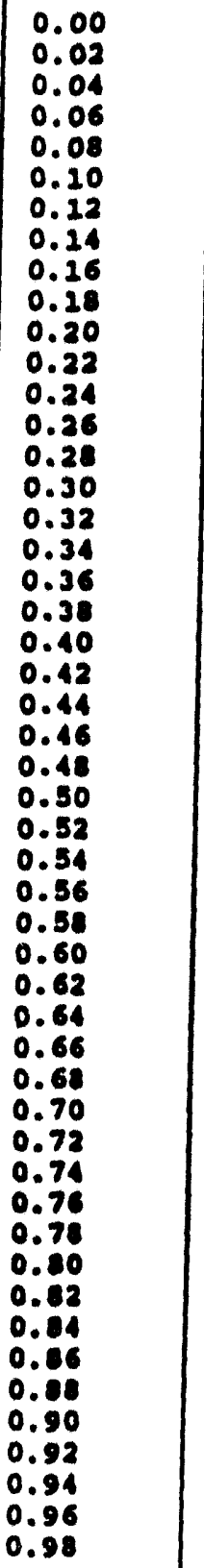 & 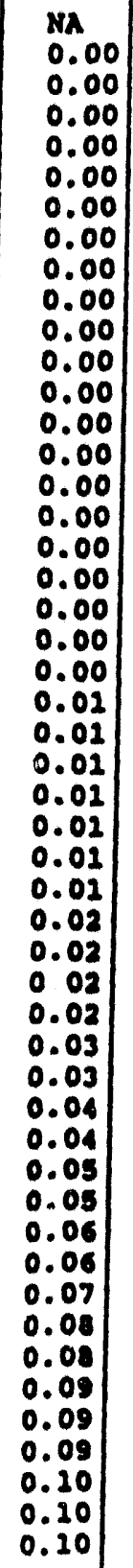 & $\begin{array}{l}\text { NA } \\
0.00 \\
0.00 \\
0.00 \\
0.00 \\
0.00 \\
0.00 \\
0.00 \\
0.00 \\
0.00 \\
0.00 \\
0.00 \\
0.00 \\
0.00 \\
0.00 \\
0.00 \\
0.00 \\
0.00 \\
0.00 \\
0.00 \\
0.00 \\
0.00 \\
0.03 \\
0.00 \\
0.00 \\
0.01 \\
0.01 \\
0.01 \\
0.01 \\
0.01 \\
0.01 \\
0.01 \\
0.02 \\
0.02 \\
0.02 \\
0.03 \\
0.03 \\
0.00 \\
0.05 \\
0.06 \\
0.07 \\
0.08 \\
0.10 \\
0.11 \\
0.12 \\
0.13 \\
0.14 \\
0.24 \\
0.15 \\
0.15\end{array}$ & 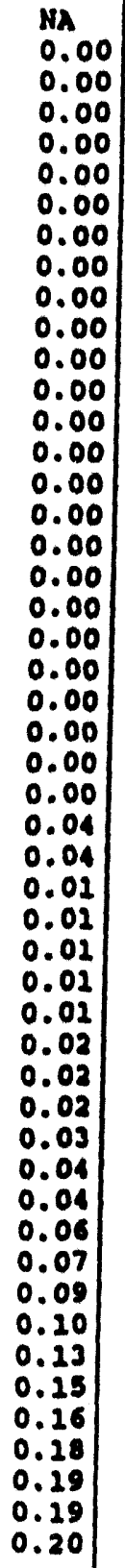 & 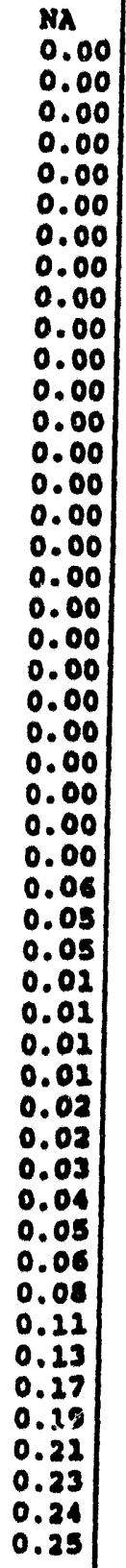 &  & $\begin{array}{l}N A \\
0.00 \\
0.00 \\
0.00 \\
0.00 \\
0.00 \\
0.00 \\
0.00 \\
0.000 \\
0.00 \\
0.00 \\
0.00 \\
0.00 \\
0.00 \\
0.00 \\
0.00 \\
0.00 \\
0.00 \\
0.00 \\
0.00 \\
0.00 \\
0.00 \\
0.00 \\
0.00 \\
0.00 \\
0.00 \\
0.00 \\
0.00 \\
0.00 \\
0.00 \\
0.00 \\
0.08 \\
0.07 \\
0.07 \\
0.06 \\
0.02 \\
0.02 \\
0.02 \\
0.03 \\
0.03 \\
0.05 \\
0.07 \\
0.10 \\
0.23 \\
0.18 \\
0.24 \\
0.28 \\
0.32 \\
0.31 \\
0.35\end{array}$ \\
\hline
\end{tabular}


Table 3.3.2-1 a Homologous Torque at $p_{1}=3 \mathrm{~atm}$ (AC Motor)

Homologous Flow Parameter $\nu / \alpha_{N}$

\begin{tabular}{|c|c|c|c|c|c|c|}
\hline pld & 0.400 & 0.600 & 0.800 & 1.000 & 1.200 & 1.400 \\
\hline $\begin{array}{l}0.000 \\
0.020 \\
0.040 \\
0.060 \\
0.080 \\
0.100 \\
0.120 \\
0.140 \\
0.160 \\
0.180 \\
0.200 \\
0.220 \\
0.240 \\
0.260 \\
0.280 \\
0.300 \\
0.320 \\
0.340 \\
0.360 \\
0.380 \\
0.400 \\
0.120 \\
0.440 \\
0.160 \\
0.480 \\
0.500 \\
0.520 \\
0.540 \\
0.560 \\
0.580 \\
0.600 \\
0.620 \\
0.610 \\
0.660 \\
0.680 \\
0.700 \\
0.720 \\
0.740 \\
0.760 \\
0.780 \\
0.800 \\
0.820 \\
0.840 \\
0.860 \\
0.880 \\
0.900 \\
0.920 \\
0.940 \\
0.960 \\
0.980 \\
0.980\end{array}$ & $\begin{array}{l}0.687 \\
0.687 \\
0.681 \\
0.681 \\
0.681 \\
0.682 \\
0.679 \\
0.680 \\
0.676 \\
0.671 \\
0.670 \\
0.671 \\
0.668 \\
0.217 \\
0.206 \\
0.198 \\
0.193 \\
0.187 \\
0.182 \\
0.177 \\
0.177 \\
0.170 \\
0.169 \\
0.165 \\
0.164 \\
0.160 \\
0.160 \\
0.156 \\
0.156 \\
0.156 \\
0.156 \\
0.153 \\
0.153 \\
0.151 \\
0.151 \\
0.151 \\
0.152 \\
0.119 \\
0.150 \\
0.151 \\
0.116 \\
0.148 \\
0.150 \\
0.152 \\
0.145 \\
0.147 \\
0.151 \\
0.158 \\
0.139 \\
0.146 \\
0.146 \\
0\end{array}$ & $\begin{array}{l}0.799 \\
0.802 \\
0.801 \\
0.802 \\
0.801 \\
0.805 \\
0.803 \\
0.801 \\
0.806 \\
0.802 \\
0.801 \\
0.801 \\
0.803 \\
0.799 \\
0.144 \\
0.101 \\
0.359 \\
0.3222 \\
0.289 \\
0.264 \\
0.239 \\
0.220 \\
0.205 \\
0.190 \\
0.179 \\
0.170 \\
0.161 \\
0.154 \\
0.1177 \\
0.114 \\
0.139 \\
0.134 \\
0.133 \\
0.129 \\
0.125 \\
0.125 \\
0.125 \\
0.121 \\
0.122 \\
0.119 \\
0.120 \\
0.117 \\
0.119 \\
0.121 \\
0.117 \\
0.121 \\
0.114 \\
0.120 \\
0.131 \\
0.117 \\
0\end{array}$ & $\begin{array}{l}0.908 \\
0.915 \\
0.913 \\
0.915 \\
0.919 \\
0.921 \\
0.923 \\
0.926 \\
0.929 \\
0.931 \\
0.928 \\
0.931 \\
0.933 \\
0.931 \\
0.932 \\
0.692 \\
0.653 \\
0.606 \\
0.557 \\
0.505 \\
0.147 \\
0.398 \\
0.351 \\
0.308 \\
0.272 \\
0.241 \\
0.219 \\
0.197 \\
0.178 \\
0.165 \\
0.151 \\
0.1141 \\
0.135 \\
0.128 \\
0.122 \\
0.116 \\
0.1111 \\
0.110 \\
0.106 \\
0.107 \\
0.101 \\
0.105 \\
0.102 \\
0.105 \\
0.102 \\
0.098 \\
0.103 \\
0.098 \\
0.109 \\
0.100 \\
0\end{array}$ & $\begin{array}{l}1.000 \\
1.009 \\
1.010 \\
1.013 \\
1.023 \\
1.027 \\
1.031 \\
1.035 \\
1.033 \\
1.038 \\
1.011 \\
1.045 \\
1.050 \\
1.017 \\
1.051 \\
1.047 \\
1.014 \\
0.072 \\
0.780 \\
0.737 \\
0.691 \\
0.631 \\
0.574 \\
0.511 \\
0.154 \\
0.393 \\
0.316 \\
0.302 \\
0.265 \\
0.236 \\
0.210 \\
0.189 \\
0.174 \\
0.162 \\
0.151 \\
0.110 \\
0.136 \\
0.130 \\
0.121 \\
0.120 \\
0.116 \\
0.117 \\
0.114 \\
0.111 \\
0.114 \\
0.112 \\
0.108 \\
0.117 \\
0.115 \\
0.150 \\
0\end{array}$ & $\begin{array}{l}1.037 \\
1.046 \\
1.017 \\
1.058 \\
1.063 \\
1.069 \\
1.075 \\
1.080 \\
1.086 \\
1.091 \\
1.097 \\
1.103 \\
1.102 \\
1.108 \\
1.111 \\
1.112 \\
1.104 \\
1.089 \\
1.067 \\
1.005 \\
0.852 \\
0.808 \\
0.761 \\
0.703 \\
0.610 \\
0.581 \\
0.513 \\
0.153 \\
0.398 \\
0.353 \\
0.313 \\
0.201 \\
0.259 \\
0.236 \\
0.223 \\
0.207 \\
0.197 \\
0.189 \\
0.182 \\
0.176 \\
0.171 \\
0.171 \\
0.170 \\
0.167 \\
0.163 \\
0.171 \\
0.169 \\
0.165 \\
0.158 \\
0.199 \\
0\end{array}$ & $\begin{array}{l}0.950 \\
0.960 \\
0.961 \\
0.972 \\
0.978 \\
0.984 \\
0.990 \\
0.897 \\
1.002 \\
1.009 \\
1.016 \\
1.023 \\
1.030 \\
1.030 \\
1.037 \\
1.014 \\
1.039 \\
1.011 \\
1.033 \\
1.022 \\
1.003 \\
0.861 \\
0.831 \\
0.802 \\
0.756 \\
0.713 \\
0.659 \\
0.602 \\
0.551 \\
0.502 \\
0.157 \\
0.129 \\
0.397 \\
0.376 \\
0.357 \\
0.339 \\
0.331 \\
0.321 \\
0.318 \\
0.311 \\
0.299 \\
0.291 \\
0.303 \\
0.299 \\
0.296 \\
0.293 \\
0.287 \\
0.279 \\
0.313 \\
0.315\end{array}$ \\
\hline
\end{tabular}


Table 3.3.2-1b Homologous Torque at $p_{1}=2 \mathrm{~atm}$ (AC Motor)

\begin{tabular}{|c|c|c|c|c|c|c|}
\hline \multirow{2}{*}{$\begin{array}{l}\text { Iniet } \\
\text { Vold }\end{array}$} & \multicolumn{6}{|c|}{ Homologous Flow Parameter $\nu / \alpha_{N}$} \\
\hline & 0.100 & 0.600 & 0.800 & 1.000 & 1.200 & 1.400 \\
\hline 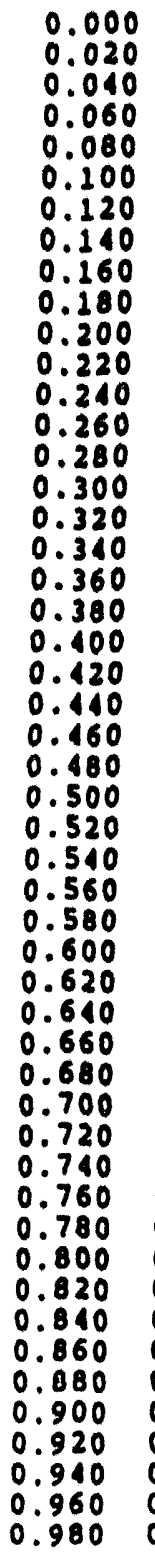 & $\begin{array}{l}0.687 \\
0.688 \\
0.689 \\
0.690 \\
0.688 \\
0.689 \\
0.690 \\
0.688 \\
0.689 \\
0.687 \\
0.688 \\
0.686 \\
0.687 \\
0.219 \\
0.210 \\
0.201 \\
0.194 \\
0.188 \\
0.182 \\
0.177 \\
0.175 \\
0.170 \\
0.169 \\
0.164 \\
0.161 \\
0.160 \\
0.159 \\
0.156 \\
0.156 \\
0.156 \\
0.156 \\
0.153 \\
0.153 \\
0.151 \\
0.151 \\
0.151 \\
0.132 \\
0.148 \\
0.149 \\
0.151 \\
0.146 \\
0.147 \\
0.149 \\
0.152 \\
0.144 \\
0.116 \\
0.150 \\
0.157 \\
0.137 \\
0.142 \\
0.142\end{array}$ & $\begin{array}{l}0.799 \\
0.801 \\
0.803 \\
0.805 \\
0.812 \\
0.815 \\
0.817 \\
0.819 \\
0.817 \\
0.820 \\
0.823 \\
0.825 \\
0.823 \\
0.826 \\
0.190 \\
0.135 \\
0.389 \\
0.316 \\
0.308 \\
0.278 \\
0.250 \\
0.229 \\
0.210 \\
0.193 \\
0.181 \\
0.171 \\
0.162 \\
0.154 \\
0.118 \\
0.1141 \\
0.139 \\
0.131 \\
0.132 \\
0.128 \\
0.128 \\
0.121 \\
0.124 \\
0.121 \\
0.1212 \\
0.119 \\
0.120 \\
0.116 \\
0.118 \\
0.120 \\
0.116 \\
0.119 \\
0.113 \\
0.118 \\
0.129 \\
0.112 \\
0.12 \\
0\end{array}$ & $\begin{array}{l}0.908 \\
0.917 \\
0.920 \\
0.924 \\
0.928 \\
0.932 \\
0.936 \\
0.939 \\
0.9914 \\
0.947 \\
0.952 \\
0.956 \\
0.960 \\
0.961 \\
0.962 \\
0.914 \\
0.723 \\
0.678 \\
0.629 \\
0.570 \\
0.509 \\
0.150 \\
0.388 \\
0.338 \\
0.296 \\
0.259 \\
0.227 \\
0.203 \\
0.182 \\
0.167 \\
0.155 \\
0.145 \\
0.136 \\
0.128 \\
0.122 \\
0.116 \\
0.114 \\
0.110 \\
0.106 \\
0.106 \\
0.103 \\
0.104 \\
0.101 \\
0.103 \\
0.101 \\
0.097 \\
0.101 \\
0.096 \\
0.106 \\
0.094 \\
0\end{array}$ & 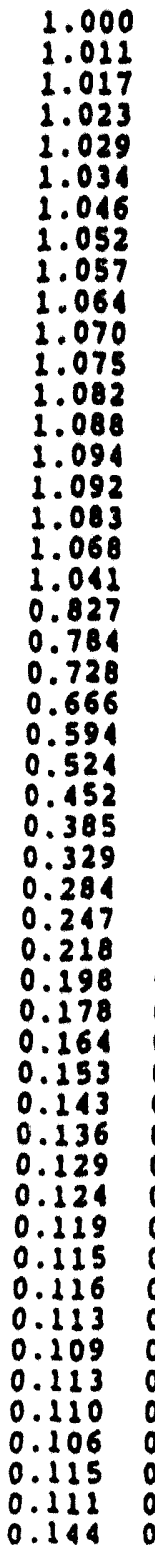 & $\begin{array}{l}1.037 \\
1.017 \\
1.055 \\
1.062 \\
1.070 \\
1.083 \\
1.090 \\
1.097 \\
1.104 \\
1.112 \\
1.121 \\
1.135 \\
1.113 \\
1.150 \\
1.151 \\
1.160 \\
1.159 \\
1.153 \\
1.151 \\
1.137 \\
1.1112 \\
0.911 \\
0.867 \\
0.817 \\
0.751 \\
0.677 \\
0.601 \\
0.518 \\
0.150 \\
0.389 \\
0.336 \\
0.300 \\
0.271 \\
0.216 \\
0.225 \\
0.212 \\
0.197 \\
0.189 \\
0.182 \\
0.176 \\
0.171 \\
0.172 \\
0.169 \\
0.165 \\
0.162 \\
0.170 \\
0.167 \\
0.163 \\
0.156 \\
0.195 \\
0\end{array}$ & $\begin{array}{l}0.950 \\
0.961 \\
0.969 \\
0.975 \\
0.982 \\
0.995 \\
1.002 \\
1.010 \\
1.021 \\
1.032 \\
1.041 \\
1.049 \\
1.058 \\
1.067 \\
1.075 \\
1.081 \\
1.092 \\
1.087 \\
1.093 \\
1.090 \\
1.091 \\
1.081 \\
1.060 \\
0.896 \\
0.859 \\
0.816 \\
0.763 \\
0.695 \\
0.621 \\
0.556 \\
0.501 \\
0.152 \\
0.415 \\
0.389 \\
0.368 \\
0.3188 \\
0.332 \\
0.325 \\
0.3199 \\
0.313 \\
0.299 \\
0.295 \\
0.303 \\
0.299 \\
0.296 \\
0.292 \\
0.287 \\
0.279 \\
0.313 \\
0.315\end{array}$ \\
\hline
\end{tabular}


Table 3.3.2-1c Homologous Torque at $p_{1}=1 \mathrm{~atm}$ (AC Motor)

Homologous Flow Parameter $\nu / \alpha_{N}$

\begin{tabular}{|c|c|c|c|c|c|c|}
\hline Vold & 0.400 & 0.600 & 0.800 & 1.000 & 1.200 & 1.100 \\
\hline $\begin{array}{l}0.000 \\
0.020 \\
0.010 \\
0.060 \\
0.080 \\
0.100 \\
0.120 \\
0.110 \\
0.160 \\
0.180 \\
0.200 \\
0.220 \\
0.210 \\
0.260 \\
0.280 \\
0.300 \\
0.320 \\
0.340 \\
0.360 \\
0.380 \\
0.100 \\
0.120 \\
0.4140 \\
0.160 \\
0.180 \\
0.500 \\
0.520 \\
0.540 \\
0.560 \\
0.580 \\
0.600 \\
0.620 \\
0.640 \\
0.660 \\
0.680 \\
0.700 \\
0.720 \\
0.7100 \\
0.760 \\
0.780 \\
0.800 \\
0.820 \\
0.810 \\
.860 \\
.880 \\
.900 \\
.920 \\
.940 \\
.960 \\
.980 \\
0.900 \\
0\end{array}$ & $\begin{array}{l}0.687 \\
0.692 \\
0.691 \\
0.697 \\
0.699 \\
0.701 \\
0.701 \\
0.706 \\
0.709 \\
0.711 \\
0.710 \\
0.113 \\
0.116 \\
0.227 \\
0.214 \\
0.204 \\
0.197 \\
0.190 \\
0.183 \\
0.180 \\
0.1775 \\
0.171 \\
0.169 \\
0.165 \\
0.164 \\
0.160 \\
0.159 \\
0.159 \\
0.156 \\
0.156 \\
0.156 \\
0.153 \\
0.153 \\
0.154 \\
0.150 \\
0.151 \\
0.152 \\
0.118 \\
0.1199 \\
0.150 \\
0.145 \\
0.147 \\
0.149 \\
0.151 \\
0.143 \\
0.146 \\
0.110 \\
0\end{array}$ & $\begin{array}{l}0.799 \\
0.806 \\
0.810 \\
0.811 \\
0.822 \\
0.825 \\
0.830 \\
0.839 \\
0.813 \\
0.817 \\
0.851 \\
0.856 \\
0.861 \\
0.866 \\
0.870 \\
0.527 \\
0.169 \\
0.110 \\
0.361 \\
0.315 \\
0.277 \\
0.217 \\
0.221 \\
0.204 \\
0.191 \\
0.176 \\
0.165 \\
0.157 \\
0.149 \\
0.1145 \\
0.139 \\
0.139 \\
0.132 \\
0.128 \\
0.128 \\
0.124 \\
0.124 \\
0.121 \\
0.121 \\
0.1218 \\
0.119 \\
0.115 \\
0.115 \\
0.120 \\
0.115 \\
0.118 \\
0.111 \\
0.116 \\
0.126 \\
0.106 \\
0\end{array}$ & 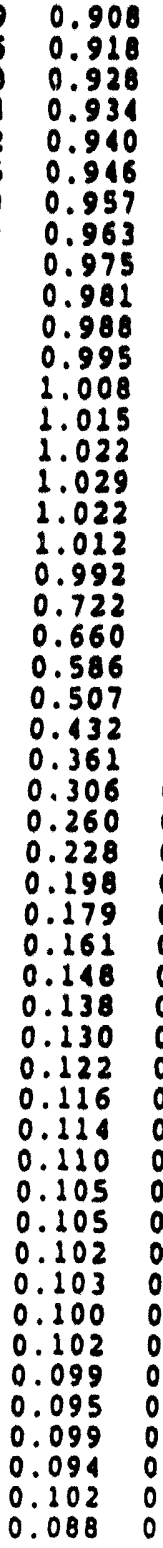 & $\begin{array}{l}1.000 \\
1.014 \\
1.027 \\
1.035 \\
1.013 \\
1.056 \\
1.065 \\
1.079 \\
1.088 \\
1.097 \\
1.112 \\
1.121 \\
1.131 \\
1.1110 \\
1.157 \\
1.167 \\
1.169 \\
1.174 \\
1.171 \\
1.171 \\
1.166 \\
1.133 \\
0.871 \\
0.801 \\
0.722 \\
0.626 \\
0.1523 \\
0.130 \\
0.319 \\
0.1294 \\
0.219 \\
0.216 \\
0.191 \\
0.170 \\
0.156 \\
0.115 \\
0.137 \\
0.129 \\
0.121 \\
0.1119 \\
0.111 \\
0.115 \\
0.112 \\
0.108 \\
0.112 \\
0.108 \\
0.105 \\
0.112 \\
0.107 \\
0.135 \\
0\end{array}$ & $\begin{array}{l}1.037 \\
1.050 \\
1.065 \\
1.071 \\
1.081 \\
1.100 \\
1.110 \\
1.126 \\
1.137 \\
1.151 \\
1.161 \\
1.176 \\
1.191 \\
1.206 \\
1.219 \\
1.230 \\
1.250 \\
1.255 \\
1.256 \\
1.263 \\
1.270 \\
1.273 \\
1.260 \\
1.235 \\
0.988 \\
0.924 \\
0.811 \\
0.739 \\
0.625 \\
0.511 \\
0.127 \\
0.350 \\
0.300 \\
0.266 \\
0.2140 \\
0.218 \\
0.205 \\
0.190 \\
0.182 \\
0.175 \\
0.170 \\
0.172 \\
0.168 \\
0.161 \\
0.161 \\
0.168 \\
0.165 \\
0.160 \\
0.152 \\
0.188 \\
0\end{array}$ & $\begin{array}{l}0.950 \\
0.962 \\
0.975 \\
0.985 \\
0.995 \\
1.009 \\
1.018 \\
1.034 \\
1.041 \\
1.061 \\
1.070 \\
1.088 \\
1.100 \\
1.111 \\
1.130 \\
1.112 \\
1.151 \\
1.162 \\
1.171 \\
1.186 \\
1.196 \\
1.206 \\
1.207 \\
1.212 \\
1.205 \\
1.187 \\
0.985 \\
0.931 \\
0.850 \\
0.756 \\
0.614 \\
0.514 \\
0.172 \\
0.119 \\
0.389 \\
0.357 \\
0.314 \\
0.327 \\
0.320 \\
0.315 \\
0.310 \\
0.306 \\
0.303 \\
0.299 \\
0.295 \\
0.292 \\
0.286 \\
0.277 \\
0.312 \\
0.314\end{array}$ \\
\hline
\end{tabular}


Table 3.3.2-1d Homologous Torque at $p_{1}=0.05$ atm (AC Motor)

Homologous Flow Parameter $\nu / \alpha_{N}$

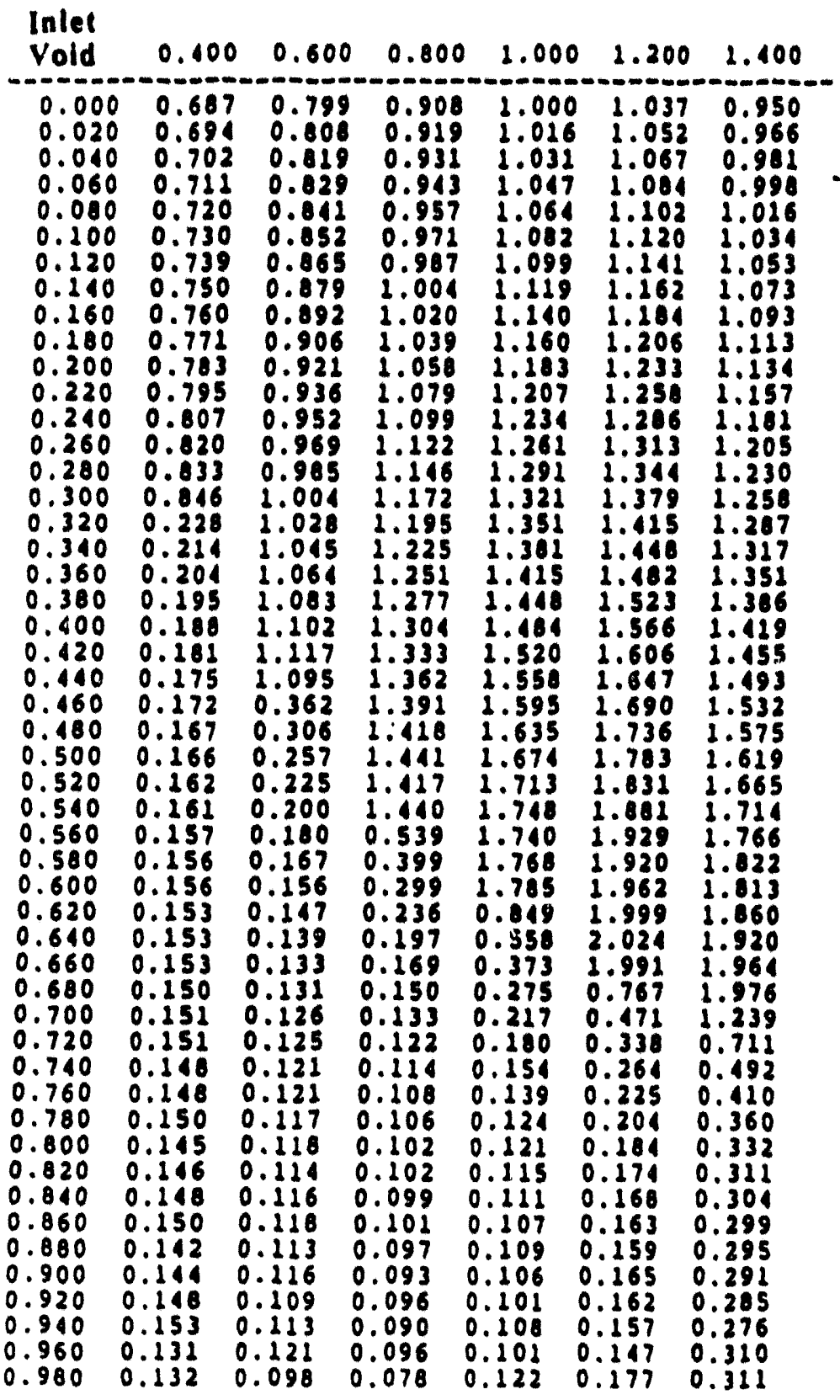


Inlet Void

Fraction

$a_{1}$

0.00

0.02

0.04

0.06

0.08

0.10

0.12

0.14

0.16

0.18

0.20

0.22

0.24

0.26

0.28

0.30

0.32

0.34

0.36

0.38

0.40

0.42

0.44

0.46

0.48

0.50

0.52

0.54

0.56

0.58

0.60

0.62

0.64

0.66

0.68

0.70

0.72

0.74

0.76

0.78

0.80

0.82

0.84

0.86

0.88

0.90

0.92

0.94

0.96

0.98

0.00
0.2

$259.688 \quad 250.569$

256.988

254.551

252.115

249.418

246.197

242.191

237.138

230.776

8.397

6.511

5.765

5.192

4.682

4.273

3.885

3.547

3.256

2.994

2.756

2.538

2.339

2.157

1.991

1.838

1.698

1.571

1.455

1.350

1.253

1.165

1.086

1.014

0.948

0.889

0.836

0.788

0.744

0.705

0.669

0.637

0.608

0.581

0.557

0.535

0.514

0.495

0.477

0.460

0.443

0.000

$$
0.4
$$

244.429

223.120

219.746

37.478

31.861

23.520

17.644

15.339

13.351

11.633

10.139

8.840

7.706

6.716

5.850

5.093

4.430

3.850

3. 344

2.902

2.518

2.185

1.898

1.649

1.436

1.254

1. 101

0.971

0.863

0.773

0.699

0.640

0.591

0.553

0.521

0.496

0.475

0.456

0.000

Table 3.5.2-1a

$\left(p_{1}=\right.$

(m)

Statlc Pressure Increase, $\left(p_{2}-p_{1}\right)$ (psi)

\section{Homologous Parameter}

0.6

0.8

1.0

1.2

1.4

$\begin{array}{llllll}238.569 & 220.437 & 192.918 & 152.761 & 96.712\end{array}$

$\begin{array}{lllll}237.467 & 219.959 & 192.995 & 153.280\end{array}$

97.385

98.002

$\begin{array}{llllll}246.580 & 236.266 & 219.386 & 192.984 & 153.720\end{array}$

234.969

218.724

192.889154 .086

$233.572 \quad 217.966 \quad 192.705 \quad 154.375$

$232.070 \quad 217.108 \quad 192.427 \quad 154.583$

$230.459216 .146 \quad 192.050 \quad 154.705$

228.733

215.072

$191.570 \quad 154.737$

226.887

213.883

190.981154 .675

224.916

222.815

212.569

211.121

18

190.278 154.513

220.576

218.182

207.8

215.555

205.892

\section{7}

134.959

203.738

115.485

195.916

97.219

185.979

$80.798 \quad 249.313$

66.754

136.507
55.070

45.498

37.725

31.368

26.147

21.840

18.270

15.294

12.802

10.709

8.947

7.460

6.206

5.147

4.256

3.506

2.880

2. 358

1.926

1.572

1.286

1.056

0.876

0.736

0.631

0.556

0.505

0.471

0.453

0.444

0.442

0.000
121.755

105.473

88.743

72.976

59.208

47.781

38.585

31.171

25.200

20.385

16.485

13.311

10.720

8.600

6.864

5.444

4.285

3. 344

2.585

1.979

1.499

1.129

0.848

0.644

0.501

0.408

0.358

0.338

0.343

0.365

0.396

0.000

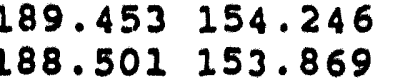

$187.414 \quad 153.373$

186.167

184.719

182.987

177.937

173.429

167.354

158.239

131.016

220.301

107.845

93.737

78.690

64.076

51.152

40.496

31.950

25.128

19.728

15.443

12.035

9.317

7.150 
$v_{1}$ Liquild Flow Velocity (feet/sec)

Inlet Vold

Fraction$$
a_{1}
$$

0.00

0.02

0.04

0.06

0.08

0.10

0.12

0.14

0.16

0.18

0.20

0.22

0.24

0.26

0.28

0.30

0.32

0.34

0.36

0.38

0.40

0.42

0.44

0.46

0.48

0.50

0.52

0.54

0.56

0.58

0.60

0.62

0.64

0.66

0.68

0.70

0.72

0.74

0.76

0.78

0.80

0.82

0.84

0.86

0.88

0.90

0.92

0.94

0.96

0.98

0.00
0.2

$$
9.078
$$

8.930

8.780

8.630

8.479

8.329

8.180

8.031

7.885

8.612

8.612

8.585

8.558

8.515

8.507

8.475

8.454

8.438

8.418

8.398

8.377

8.357

8.337

8.317

8.297

8.277

8.257

8.238

8.218

8.199

8.180

8.162

8.144

8.126

8.108

8.089

8.070

8.050

8.031

8.011

7.991

7.970

7.949

7.928

7.907

7.886

7.865

7.845

7.825

7.805

0.000
0.4

18.155

17.850

17.547

17.245

16.945

16.647

16.351

26.056

15.763

25.472

15.184

14.896

14.611

15.644

15.597

25.575

15.568

15.572

15.586

15.607

15.634

15.665

15.699

25.735

15.770

15.805

15.838

15.869

15.897

15.922

15.943

15.960

15.972

15.981

15.984

15.984

15.978

15.969

15.955

15.936

15.914

15.887

15.858

15.825

15.790

15.753

15.715

15.675

15.636

15.597

0.000

\section{Homologous Parameter}

$$
\begin{array}{ll}
0.6 & 0.8
\end{array}
$$

1.0

1.2

1.4

27.233

26.773

26.315

25.861

25.410

24.962

24.514

24.072

23.631

23.194

22.758

22.327

21.897

21.470

21.293

21.080

20.958

20.929

20.977

21.080

21.208

21.200

21.285

21.456

21.653

21.862

22.076

22.289

22.496

22.692

22.876

23.045

23.198

23.333

23.450

23.549

23.630

23.692

23.737

23.764

23.776

23.772

23.754

23.723

23.682

23.632

23.575

23.512

23.446

23.378

0.000

36.311

35.693

35.075

34.456

33.839

33.222

32.607

31.992

31.379

30.779

30.199

29.626

29.067

28.506

27.951

27.432

26.940

26.500

26.112

25.814

25.644

25.632

25.786

26.075

26.437

26.567

26.896

27.345

27.825

28.312

28.789

29.246

29.673

30.064

30.415

30.723

30.987

31.207

31.383

31.517

31.609

31.664

31.683

31.670

31.630

31.565

31.481

31.382

31.273

31.157

0.000

45.389

54.466

53.559

52.648

51.737

50.826

49.915

49.004

48.094

47.184

46.276

45.369

44.464

.43 .561

42.662

41.766

40.875

40.013

39.156

38.315 
Inlet Vold

Fraction

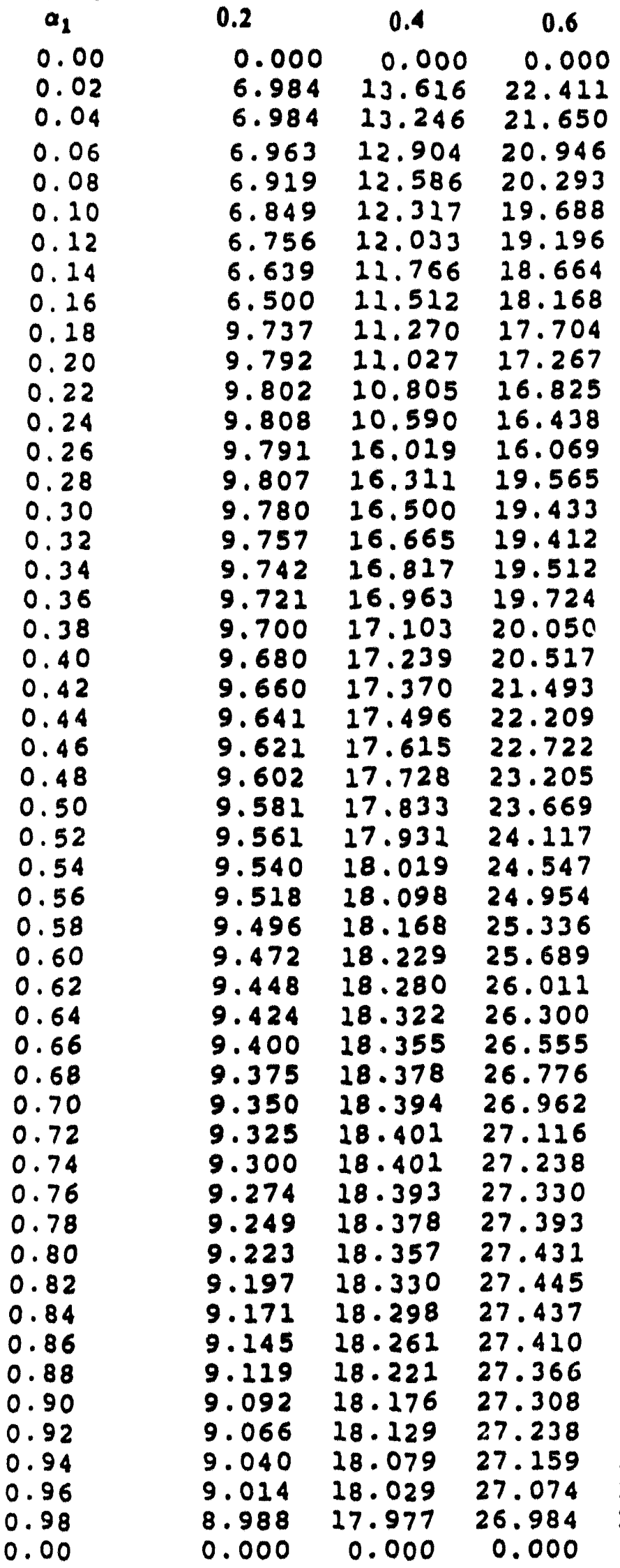

vas Flow Velocity (feet/sec)

Homolozous Parameter

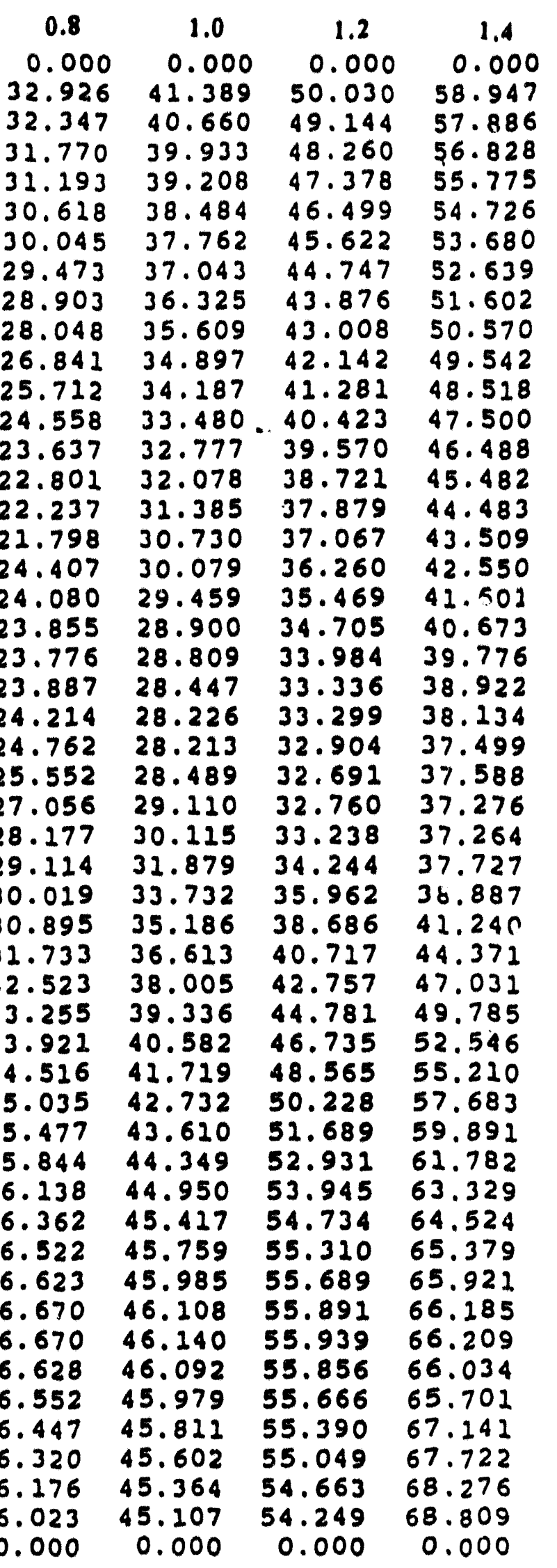


Inlet Void

Fraction

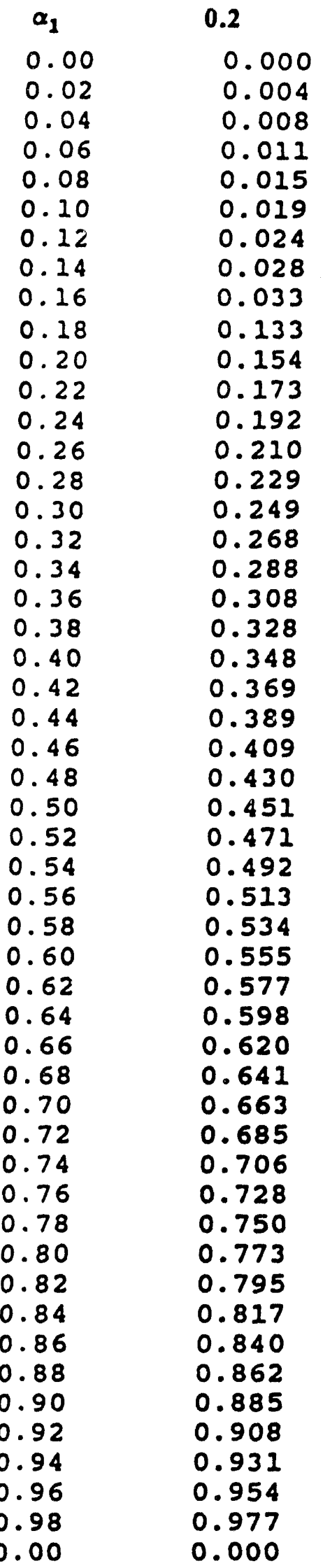

$\alpha_{2}$ Void Fraction

Homologous Parameter

\section{4 \\ 0.6}

0.000

0.000

0.003

0.8

0.003

0.007

0.006

0.010

0.010

0.014

0.014

0.018

0.023

0.022

0.027

0.027

0.032

0.037

0.038

0.043

0.049

0.055

0.043

0.049

0.055

0.141

0.162

0.061

0.078

0.096

0.207

0.118

0.143

0.171

0.200

0.230

0.256

0.284

0.315

0.346

0.377

0.408

0.438

0.467

0.496

0.524

0.551

0.577

0.603

0.628

0.653

0.677

0.701

0.725

0.748

0.77 .1

0.794

0.817

0.000

0.003

0.006

0.009

0.013

0.016

0.020

0.024

0.028

0.033

0.038

0.044

0.051

0.057

0.065

0.073

0.083

0.095

0.109

0.127

0.150

0.178

0.212

0.248

0.286

0.317

0.352

0.389

0.426

0.461

0.496

0.528

0.559

0.589

0.618

0.645

0.672

0.697

0.722

0.747

0.770

0.794

0.817

0.839

0.839

0.862

0.885

0.862

0.885

0.908

0.931

0.954

0.977

0.000
1.0

0.000

0.003

0.006

0.010

0.013

0.017

0.021

0.025

0.029

0.033

0.038

0.043

0.048

0.054 .

0.059

0.065

0.073

0.081

0.090

0.102

0.125

0.142

0.164

0.190

0.223

0.262

0.305

0.342

0.380

0.422

0.463

0.502

0.539

0.574

0.607

0.637

0.666

0.694

0.720

0.746

0.770

0.794

0.817

0.840

0.863

0.885

0.908

0.931

0.954

0.977

0.000
1.2

0.000

0.003

0.007

0.010

0.014

0.018

0.022

0.026

0.030

0.035

0.040

0.044

0.050

0.055

0.061

0.067

0.074

0.082

0.090

0.099

0.110

0.123

0.149

0.169

0.192

0.222

0.258

0.301

0.346

0.384

0.430

0.475

0.518

0.558

0.595

0.630

0.662

0.691

0.719

0.746

0.771

0.795

0.818

0.841

0.864

0.886

0.909

0.931

0.954

0.977

0.000
1.4

0.000

0.004

0.007

0.011

0.015

0.019

0.023

0.027

0.032

0.036

0.041

0.046

0.051

0.057

0.063

0.069

0.076

0.083

0.091

0.100

0.110

0.121

0.134

0.151

0.180

0.203

0.231

0.267

0.311

0.355

0.398

0.449

0.497

0.543

0.584

0.623

0.658

0.690

0.720

0.747

0.773

0.797

0.820

0.843

0.865

0.887

0.949

0.962

0.975

0.987

0.000 
Table 3.5.2-1b $\quad\left(p_{1}=2 \mathrm{~atm}\right)$

Static Pressure Increase, $\left(p_{2}-p_{1}\right)$ (psi)

\begin{tabular}{|c|c|c|c|c|c|c|c|}
\hline \multirow{2}{*}{$\begin{array}{l}\text { Inlet Void } \\
\text { Fraction } \\
\alpha_{1}\end{array}$} & \multicolumn{7}{|c|}{ Homologous Parameter } \\
\hline & 0.2 & 0.4 & 0.6 & 0.8 & 1.0 & 1.2 & 1.4 \\
\hline 0.00 & 259.688 & 250.569 & 569 & 220.437 & 192 & 152.761 & 96.712 \\
\hline 0.02 & 259.530 & 249.230 & 237.995 & 220.439 & 193.440 & 153.676 & 97.663 \\
\hline 0.04 & 258.213 & 247.779 & 237.323 & 220.368 & 193.896 & 154.518 & 98.562 \\
\hline 0.06 & 255.999 & 246.231 & 236.568 & 220.219 & 194.281 & 155.298 & 99.417 \\
\hline 0.08 & 253.154 & 244.581 & .235 .727 & 2.29 .988 & 194.590 & 156.012 & 100.226 \\
\hline 0.10 & 249.942 & 242.824 & 234.792 & 219.672 & 194.820 & 156.658 & 100.987 \\
\hline 2 & 246.623 & 240.956 & 233.760 & 219.264 & 194.965 & 157.230 & 101.696 \\
\hline 0.14 & 243.462 & 238.973 & 232.624 & 218.761 & 195.020 & 157.726 & 102.350 \\
\hline 0 & 240.724 & 236.871 & 231.381 & 218.155 & 194.979 & 158.141 & 102.948 \\
\hline 0.18 & 38.670 & 234.644 & 230.023 & 217.441 & 194.839 & 158.467 & 103.485 \\
\hline 0.20 & 6.467 & 232.287 & 228.543 & 216.614 & 194.593 & 158.702 & 103.958 \\
\hline 0.22 & .704 & 229.793 & 226.935 & 215.664 & 194.233 & 158.839 & 104.361 \\
\hline 24 & .118 & 227.080 & 225.186 & 214.583 & 193.755 & $158.87 j$ & 104.692 \\
\hline 26 & 599 & 39.479 & 223.241 & 213.335 & 193.136 & 258.791 & 104.943 \\
\hline 28 & 183 & 33.261 & 220.984 & 211.886 & 192.346 & 158.565 & 105.094 \\
\hline 0.30 & 790 & 28.323 & 136.036 & 207.791 & 191.332 & 158.168 & 105.131 \\
\hline 0.32 & 446 & 24.254 & 115.000 & 203.092 & 188.136 & 156.612 & 104.967 \\
\hline 0.34 & .152 & 20.863 & 932 & 196.735 & 185.441 & 155.024 & 103.826 \\
\hline .36 & 2.886 & 18.022 & 317 & 184.045 & 182.149 & 153.307 & 103.089 \\
\hline 38 & 2.645 & 15.601 & 62.676 & 145.621 & 177.756 & 151.140 & 102.134 \\
\hline 40 & .424 & 13.525 & 50.881 & 129.359 & 171.499 & 148.335 & 100.883 \\
\hline 0.42 & 2.222 & 11.737 & 41.491 & 110.464 & 161.521 & 144.633 & 99.252 \\
\hline 0.44 & 2.038 & 10.191 & 34.036 & 90.564 & 131.597 & 139.572 & 97.117 \\
\hline 46 & .870 & 8.849 & 28.030 & 72.140 & 118.101 & .263 & 94.277 \\
\hline 48 & .715 & 7.681 & 23.169 & 56.778 & 101.730 & 109.305 & 90.408 \\
\hline & 574 & 6.665 & 202 & 44. & 83.2 & 98.7 & 84.910 \\
\hline 52 & .445 & 5.778 & 940 & 35. & 65.2 & 85.600 & $69 \cdot 9,98$ \\
\hline 54 & 1.328 & 5.003 & 239 & 28 & 50.0 & 69.9 & 62.240 \\
\hline 56 & 1.220 & 4.328 & 10.993 & 6 & 38.321 & 54.040 & 52.407 \\
\hline & .124 & 3.738 & 9.117 & 17.786 & 29.361 & 473 & 41.007 \\
\hline 60 & .035 & 3.224 & 7.546 & 14. & 22.530 & 119 & 30.336 \\
\hline 62 & .955 & 776 & 6.228 & 11.290 & 17.297 & .326 & 21.985 \\
\hline 64 & 881 & 387 & 5.123 & 8.957 & .250 & 16.520 & 15.757 \\
\hline 66 & 315 & & 4.196 & 7.0 & 10.098 & 12.161 & 11.213 \\
\hline 68 & 56 & & 3.421 & 5.5 & 7.6 & 8.8 & 7.882 \\
\hline & 101 & & 2.7 & & 5.6 & 6.3 & 5.419 \\
\hline & 652 & & & & 4.1 & 4.4 & 3.589 \\
\hline & 609 & & 1.75 & & 2.9 & 2. & 2.235 \\
\hline & & & 1.43 & 1.8 & 20 & 1.9 & 1.243 \\
\hline 78 & & 0.825 & 1.14 & 1.3 & 1.3 & 1.0 & 0.535 \\
\hline & & 0.717 & & & & 0.5 & 0.048 \\
\hline & & & & & & 0.1 & -0.265 \\
\hline & & & 0.588 & & 0.247 & -0.12 & -0.444 \\
\hline & & 0.493 & 0.484 & $0.3 !$ & 0.086 & -0.265 & -0.519 \\
\hline & & 0.445 & 0.407 & 0.260 & 0.000 & -0.318 & -0.520 \\
\hline & & 0.406 & 0.356 & & -0.02 & -0.303 & -0.466 \\
\hline & 0.357 & 0.375 & 0.324 & 0.191 & -0.014 & -0.242 & 0.161 \\
\hline & & 0.349 & 0.305 & 0.197 & 0.0 & -0.14 & 0.139 \\
\hline & & 0.329 & 0.297 & 0.220 & 0.102 & -0.0 & 0.140 \\
\hline & & . & & 0. & 0. & 0.09 & 0.153 \\
\hline & 0 & 0 & 0 & Do & 0.1 & 0.000 & 0.000 \\
\hline
\end{tabular}


$v_{12}$ Liquid Flow Velocity (feet/sec)

Inlet Void

Fraction

0.00

0.02

0.04

0.06

0.08

0.10

0.12

0.14

0.16

0.18

0.20

0.22

0.24

0.26

0.28

0.30

0.32

0.34

0.36

0.38

0.40

0.42

0.44

0.46

0.48

0.50

0.52

0.54

0.56

0.58

0.60

0.62

0.64

0.66

0.68

0.70

0.72

0.74

0.76

0.78

0.80

0.82

0.84

0.86

0.88

0.90

0.92

0.94

0.96

0.98

0.00
0.2

9.078

8.913

8.750

8.588

8.428

8.269

8. 111

7.953

7.797

7.642

8.514

8.490

8.462

8.420

8.411

8.379

8.358

8.345

8.327

8.309

8.291

8.274

8.257

8.240

8.224

8.208

8.192

8.176

8.160

8.145

8.130

8. 115

8.101

8.086

8.070

8.055

8.038

8.022

8.005

7.988

7.969

7.951

7.933

7.914

7.895

7.875

7.857

7.838

7.820

7.802

0.000
0.4

18.155

17.830

17.504

17.180

16.858

16.538

16.219

15.902

15.586

15.272

14.959

14.648

14.338

15.181

15.073

15.022

14.992

14.980

14.984

15.002

15.033

15.073

15.122

15.178

15.237

15.300

15.364

15.428

15.490

15.549

15.604

15.655

15.700

15.739

15.772

15.799

15.819

15.832

15.839

15.839

15.833

15.821

15.803

15.781

15.755

15.725

15.693

15.659

15.625

15.590

0.000
Homologous Parameter

$$
0.6
$$

0.8

1.0

1.2

1.4

27.233

26.743

26.255

25.769

25.284

24.802

24.324

23.847

23.373

22.898

22.428

21.960

21.493

21.028

20.566

20.334

20.049

19.879

19.813

19.828

19.896

19.982

19.946

20.090

20.285

20.515

20.769

21.039

21.317

21.596

21.871

22.137

22.389

22.624

22.837

23.028

23.193

23.334

23.448

23.536

23.600

23.640

23.658

23.655

23.636

23.601

23.555

23.499

23.438

23.372

0.000
$36.311 \quad 45.389$

$35.661 \quad 44.582$

$35.009 \quad 43.772$

$34.358 \quad 42.963$

33.706

33.055

32.405

31.755

31.105

30.456

29.808

29.161

28.531

27.915

27.310

26.703

26.112

25.532

24.973

24.578

24.171

23.893

23.795

23.896

24.157

24.501

24.640

25.073

25.600

26.179

26.786

27.402

28.011

28.597

29.150

29.657

30.112

30.508

30.843

31.116

31.326

31.476

31.570

31.613

31.611

31.571

31.498

31.402

31.287

0.000

42.153

41.343

40.533

39.724

38.915

38.106

37.298

36.491

35.685

34.881

34.078

33.277

32.494

31.711

30.937

30.178

29.447

28.776

28.492
31.163 
$v_{\text {a }}$ Gas Flow Velocity (feet/sec)

Inlet Void

Fraction

$\alpha_{1}$
0.00
0.02
0.04
0.06
0.08
0.10
0.12
0.14
0.16
0.18
0.20
0.22
0.24
0.26
0.28
0.30
0.32
0.34
0.36
0.38
0.40
0.42
0.44
0.46
0.48
0.50
0.52
0.54
0.56
0.58
0.60
0.62
0.64
0.66
0.68
0.70
0.72
0.74
0.76
0.78
0.80
0.82
0.84
0.86
0.88
0.90
0.92
0.94
0.96
0.98
0.00

\section{2}

0.000

9.530

8.923

8.444

8.058

7.737

7.458

7.202

6.951

6.689

9.669

9.684

9.690

9.676

9.694

9.677

9.657

9.642

9.623

9.604

9.586

9.570

$9.55 !$

9.538

9.522

9.506

9.490

9.473

9.456

9.437

9.418

9.398

9.378

9.357

9.335

9.314

9.292

9.270

9.247

9.225

9.201

9.178

9.154

9.130

9.107

9.082

9.058

9.034

9.009

8.986

0.000
0.4

0.000

14.727

14.276

13.846

13.437

12.984

12.619

12.222

11.895

11.545

11.217

10.909

10.618

15.184

15.690

15.870

16.019

16.159

16.296

16.435

16.577

16.720

16.864

17.006

17.145

17.281

17.411

17.535

17.651

17.759

17.857

17.946

18.024

18.092

18.149

18.195

18.230

18.256

18.271

18.277

18.274

18.263

18.244

18.219

18.188

18.151

18.110

18.066

18.020

17.973

0.000
Homologous Parameter

$\begin{array}{ll}0.6 & 0.8\end{array}$

0.000

24.267

0.000
32.889

23.322

22.407

21.688

20.869

20.105

19.392

18.729

18.186

17.599

17.050

16.535

16.051

15.595

18.639

18.445

18.388

18.466

18.668

18.998

19.527

20.602

21.152

21.654

22.150

22.648

23.143

23.633

24.109

24.566

24.999

25.401

25.770

26.100

26.392

26.644

21.855

27.026

27.159

27.256

27.318

27.350

27.354

27.332

27.290

27.231

27.157

27.074

26.984

0.000
32.279

31.6 .70

31.061

30.453

29.846

29.240

28.635

28.032

27.429

26.828

25.810

24.597

23.353

22.585

21.754

21.082

20.842

22.602

22.267

22.081

22.123

22.400

22.946

23.793

25.424

26.466

27.456

28.450

29.442

30.420

31.366

32.263

33.094

33.849

34.517

35.092

35.572

35.958

36.253

36.463

36.593

36.654

36.651

36.597

36.499

36.368

36.212

36.042

0.000
1.0

0.000

41.341

40.570

39.802

39.034

38.267

37. 502

36.737

35.975

35.214

34.454

33.696

32.941

32.187

31.436

30.688

29.958

29.228

28.507

27.801

27.123

26.501

26.293

25.902

25.715

25.853

26.414

27.435

29.467

31.248

32.841

34.460

36.089

37.693

39.231

40.666

42.966

43.105

44.070

44.851

45.452

45.881

46.150

46.277

46.282

46.186

46.006

45.764

45.478

45.165

0.000

1.2

0.000

49.969

49.029

48.092

47.156

46.222

45.290

44.361

43.433

42.508

41.585

40.664

$-39.746$

38.831

37.920

37.012

36.118 
$\alpha_{2}$ Void Fraction

Inlet Void

Fraction

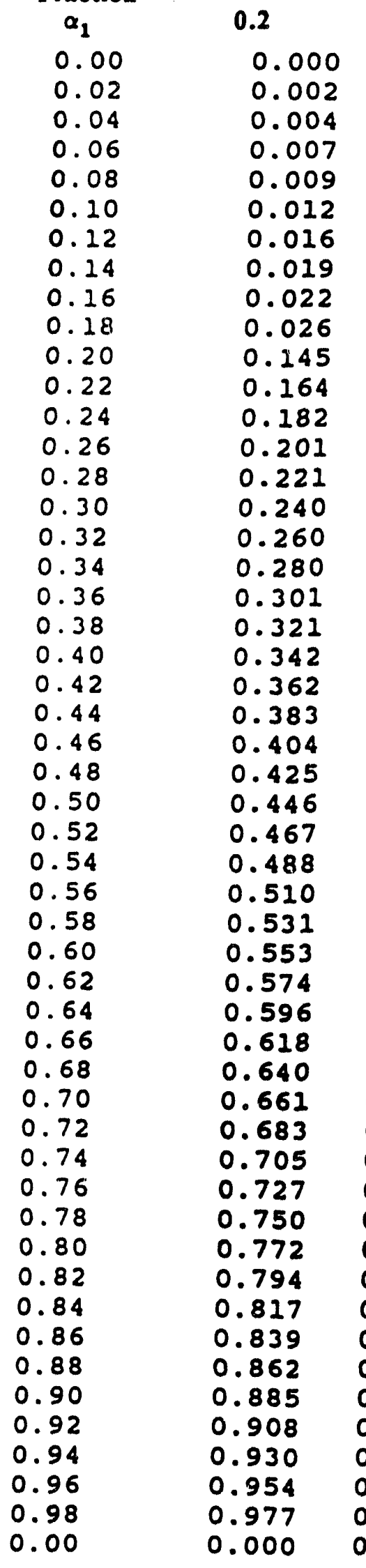

Homologous Parameter

0.4
0.000
0.002
0.004
0.007
0.009
0.012
0.015
0.018
0.021
0.025
0.029
0.033
0.038
0.114
0.132
0.153
0.176
0.200
0.224
0.249
0.275
0.301

0.6

0.8

0.000

0.002

0.004

0.007

0.009

0.012

0.015

0.018

0.021

0.025

0.029

0.033

0.037

0.042

0.046

0.061

0.078

0.099

0.123

0.251

0.180

0.211

0.236

0.268

0.302

0.337

0.371

0.405

0.438

0.470

0.502

0.533

0.562

0.591

0.618

0.645

0.671

0.697

0.721

0.745

0.769

0.793

0.816

0.839

0.862

0.885

0.908

0.930

0.954

0.977

0.000
0.000

0.002

0.004

0.007

0.009

0.011

0.014

0.017

0.019

0.022

0.025

0.029

0.033

0.037

0.043

0.048

0.054

0.061

0.070

0.083

0.097

0.118

0.146

0.180

0.219

0.260

0.293

0.334

0.376

0.418

0.458

0.496

0.533

0.568

0.601

0.633

0.662

0.691

0.717

0.743

0.768

0.792

0.816

0.839

0.862

0.885

0.908

0.931

0.954

0.977

0.000
1.0

0.000

0.002

0.005

0.007

0.009

0.012

0.015

0.017

0.020

0.023

0.026

0.030

0.033

0.037

0.041

0.045

0.050

0.055

0.061

0.067

0.075

0.085

0.107

0.125

0.148

0.181

0.223

0.270

0.312

0.358

0.407

0.454

0.500

0.542

0.582

0.619

0.653

0.685

0.714

0.742

0.768

0.793

0.817

0.840

0.863

0.886

0.908

0.931

0.954

0.977

0.000
1.2

0.000

0.002

0.005

0.007

0.010

0.012

0.015

0.018

0.021

0.024

0.027

0.031

.0 .034

0.038

0.042

0.046

0.051

0.056

0.062

0.068

0.074

0.082

0.091

0.102

0.126

0.146

0.172

0.208

0.255

0.308

0.354

0.409

0.463

0.514

0.561

0.605

0.644

0.680

0.712

0.742

0.769

0.795

0.819

0.842

0.865

0.887

0.909

0.931

0.954

0.977

0.000
1.4

0.000

0.002

0.005

0.008

0.010

0.013

0.016

0.019

0.022

0.025

0.028

0.032

0.036

0.039

0.043

0.048

0.052

0.057

0.063

0.068

0.075

0.082

0.090

0.099

0.110

0.124

0.152

0.176

0.210

0.257

0.313

0.364

0.425

0.484

0.540

0.590

0.636

0.676

0.712

0.744

0.772

0.798

0.822

0.845

0.867

0.889

0.949

0.962

0.975

0.987

0.000 
Table 3.5.2-1c ( $\quad\left(p_{1} \quad a t m\right)$

Static Pressure Increase, $\left(p_{2}-p_{1}\right)(p s i)$

Inlet Void

Fraction$$
\alpha_{1}
$$

0.00

0.02

0.04

0.06

0.08

0.10

0.12

0.14

0.16

0.18

0.20

0.22

0.24

0.26

0.28

0.30

0.32

0.34

0.36

0.38

0.40

0.42

0.44

0.46

0.48

0.50

0.52

0.54

0.56

0.58

0.60

0.62

0.64

0.66

0.68

0.70

0.72

0.74

0.76

0.78

0.80

0.82

0.84

0.86

0.88

0.90

0.92

0.94

0.96

0.98

0.00

$$
\begin{array}{lllllll}
0.2 & 0.4 & 0.6 & 0.8 & 1.0 & 1.2 & 1.4
\end{array}
$$

$\begin{array}{llllllll}259.688 & 250.569 & 238.569 & 220.437 & 192.918 & 152.761 & 96.712\end{array}$

$\begin{array}{llllllll}259.208 & 250.005 & 238.665 & 221.071 & 194.029 & 154.183 & 98.018\end{array}$

$\begin{array}{llllllll}258.447 & 249.353 & 238.685 & 221.652 & 195.093 & 155.549 & 99.282\end{array}$

$257.425 \quad 248.627 \quad 238.646 \quad 222.179 \quad 196.106 \quad 156.868 \quad 100.514$

$256.158 \quad 247.819238 .545 \quad 222.647 \quad 197.064 \quad 158.141 \quad 101.711$

$254.662 \quad 246.925 \quad 238.378 \quad 223.052 \quad 197.965 \quad 159.361 \quad 102.873$

$\begin{array}{llllllll}252.959 & 245.938 & 238.141 & 223.392 & 198.803 & 160.530 & 103.996\end{array}$

$\begin{array}{llllllll}251.063 & 244.854 & 237.829 & 223.662 & 199.577 & 161.641 & 105.079\end{array}$

$\begin{array}{llllllll}248.992 & 243.663 & 237.436 & 223.858 & 200.282 & 162.693 & 106.118\end{array}$

$246.766 \quad 242.360 \quad 236.957 \quad 223.974 \quad 200.914 \quad 163.681 \quad 107.113$

$\begin{array}{llllllll}6.447 & 240.938 & 236.385 & 224.005 & 201.468 & 164.601 & 108.059\end{array}$

$5.616 \quad 239.388 \quad 235.713223 .952 \quad 201.939 \quad 165.449108 .956$

$5.011237 .679234 .934 \quad 223.803 \quad 202.320 \quad 166.221 \quad 109.798$

$4.485235 .680234 .013 \quad 223.542 \quad 202.602 \quad 166.909 \quad 110.581$

$\begin{array}{llllllll}4.062 & 37.097 & 232.908 & 223.137 & 202.762 & 167.491 & 111.291\end{array}$

$\begin{array}{llllllll}3.664 & 31.173 & 226.411 & 222.539 & 202.775 & 167.949 & 111.913\end{array}$

$\begin{array}{llllllll}3.318 & 26.380 & 157.173 & 219.588 & 201.599 & 168.197 & 112.403\end{array}$

$\begin{array}{lllllll}3.021 & 22.465 & 132.923 & 217.437 & 200.436 & 167.160 & 112.022\end{array}$

$\begin{array}{llllllll}2.754 & 19.239 & 108.273 & 214.751 & 199.277 & 166.771 & 112.073\end{array}$

$\begin{array}{llllllll}2.511 & 16.526 & 85.531 & 210.965 & 197.753 & 166.174 & 112.024\end{array}$

$\begin{array}{llllllll}2.289 & 14.224 & 67.024 & 204.945 & 195.714 & 165.286 & 111.808\end{array}$

$\begin{array}{llllllll}2.086 & 12.260 & 52.675 & 160.068 & 192.949 & 164.033 & 111.390\end{array}$

$\begin{array}{llllllll}1.900 & 10.576 & 41.783 & 140.162 & 189.090 & 162.311 & 110.729\end{array}$

$\begin{array}{llllllll}1.730 & 9.125 & 33.486 & 114.754 & 183.332 & 159.960 & 109.769\end{array}$

$\begin{array}{lllllll}1.575 & 7.871 & 27.044 & 87.725 & 173.258 & 156.705 & 108.426\end{array}$

$\begin{array}{lllllll}1.433 & 6.786 & 21.976 & 65.118 & 135.098 & 152.041 & 106.564\end{array}$

$\begin{array}{llllllll}1.304 & 5.844 & 17.935 & 48.552 & 113.604 & 144.834 & 103.974\end{array}$

$\begin{array}{lllllll}1.186 & 5.025 & 14.675 & 36.736 & 86.335 & 117.027 & 100.253\end{array}$

$\begin{array}{lllllll}1.078 & 4.314 & 12.021 & 28.146 & 61.475 & 100.536 & 94.547\end{array}$

$\begin{array}{lllllll}0.981 & 3.696 & 9.844 & 21.753 & 43.678 & 77.196 & 75.673\end{array}$

$\begin{array}{lllllll}0.892 & 3.159 & 8.049 & 16.903 & 31.595 & 53.173 & 62.945\end{array}$

$\begin{array}{lllllll}0.811 & 2.693 & 6.564 & 13.160 & 23.150 & 36.083 & 44.679\end{array}$

$\begin{array}{lllllll}0.738 & 2.289 & 5.331 & 10.235 & 17.095 & 24.952 & 29.000\end{array}$

$\begin{array}{lllllll}0.671 & 1.940 & 4.309 & 7.927 & 12.646 & 17.460 & 19.001\end{array}$

$\begin{array}{lllllll}0.611 & 1.640 & 3.462 & 6.098 & 9.313 & 12.257 & 12.615\end{array}$

$\begin{array}{lllllll}0.557 & 1.382 & 2.762 & 4.644 & 6.785 & 8.538 & 8.377\end{array}$

$\begin{array}{lllllll}0.508 & 1.162 & 2.185 & 3.489 & 4.855 & 5.822 & 5.451\end{array}$

$\begin{array}{lllllll}0.464 & 0.975 & 1.712 & 2.577 & 3.379 & 3.817 & 3.368\end{array}$

$\begin{array}{lllllll}0.424 & 0.817 & 1.329 & 1.861 & 2.260 & 2.334 & 1.858\end{array}$

0.389

0.684

1.021

1.308

1.422

1.254

0.765

0.777

0.887

0.810

0.491

0.002

0.326

0.482

0.587

0.379

0.093

$-0.016$

$-0.483$

0.4420 .352

0.335

0.201

$-0.081$

$-0.322$

$-0.733$

0.347

0.258

0.106

$-0.168$

$-0.476$

$-0.806$

$0.260 \quad 0.207$

0.057

$-0.192$

$-0.518$

$-0.766$

0.174

0.040

$-0.167$

$-0.485$

0.125

0.203

0.156

0.048

$-0.112$

$-0.401$

0.072

0.182

0.149

0.073

$-0.037$

$-0.285$

0.05 !

0.147

0.107

0.047

$-0.154$

0.05

0.06 :

0.000

0.000

0.000

0.000

0.000

0.000 
$v_{12}$ Liquid Flow Velocity (feet/sec)

Inlet Void

Fraction

$\alpha_{1}$

0.00

0.02

0.04

0.06

0.08

0.10

0.12

0.14

0.16

0.18

0.20

0.22

0.24

0.26

0.28

0.30

0.32

0.34

0.36

0.38

0.40

0.42

0.44

0.46

0.48

0.50

0.52

0.54

0.56

0.58

0.60

0.62

0.64

0.66

0.68

0.70

0.72

0.74

0.76

0.78

0.80

0.82

0.84

0.86

0.88

0.90

0.92

0.94

0.96

0.98

0.00
0.2

9.078

8.904

8.731

8.558

8. 385

8. 212

8.040

7.869

7.697

7.527

8. 304

8.264

8.230

8.188

8.173

8.139

8.115

8.101

8.086

8.072

8.060

8.049

8.040

8.031

8.023

8.016

8.009

8.003

7.997

7.991

7.985

7.981

7.975

7.969

7.963

7.956

7.948

7.940

7.930

7.920

7.909

7.898

7.886

7.872

7.859

7.846

7.833

7.819

7.807

7.794

0.000
0.4

18.255

17.810

17.464

17.118

16.772

16.426

16.080

15.735

15.391

15.046

14.703

14.359

14.017

13.678

14.528

14.132

14.019

13.935

13.878

13.845

13.838

13.854

13.892

13.950

24.025

14.116

14.219

14.331

14.452

14.577

14.704

14.830

14.952

15.068

15.176

15.275

15.363

25.440

15.505

15.557

15.598

15.627

15.646

15.654

15.654

15.647

15.633

15.616

15.595

15.574

0.000

Homologous Parameter

$\begin{array}{lllll}0.6 & 0.8 & 1.0 & 1.2 & 1.4\end{array}$

27.233

26.717

26.200

25.683

25.166

24.649

24.132

23.615

23.099

22.582

22.069

21.561

21.054

20.551

20.044

19.541

19.198

18.818

18.343

18.311

18.029

17.959

17.930

17.825

17.876

18.004

18.197

18.448

18.752

19.100

19.484

19.895

20.320

20.750

21.173

21.579

21.958

22.303

22.608

22.869

23.083

23.252

23.376

23.458

23.503

23.516

23.503

23.469

23.420

23.363

0.000

$3-56$

$\begin{array}{ll}36.311 & 45.389\end{array}$

$35.626 \quad 44.535$

$34.938 \quad 43.679$

$\begin{array}{lll}34.251 & 42.822\end{array}$

$33.563 \quad 41.964$

32.875

32.187

31.500

30.812

30.124

29.436

28.749

28.062

27.375

26.688

26.002

25.323

24.650

23.988

23.328

22.678

22.216

21.658

21.220

21.008

21.026

21.189

21.296

21.571

22.033

22.619

23.313

24.096

24.946

25.839

26.744

27.631

28.471

29.238

29.908

30.469

30.913

31.240

31.456

31.573

31.605

31.566

31.474

31.343

31.191

0.000

41.107

40.249

39.391

38.532

37.674

36.815

35.957

35.098

34.240

33.382

32.524

31.671

30.819

29.967

29.117

28.271

27.430

26.597

25.779

24.996

24.548

24.014

23.798

23.994 


\section{Ya Gas Flow Velocity (feet/sec)}

Inlet Void

Fraction

\begin{tabular}{|c|c|c|}
\hline$\alpha_{1}$ & 0.2 & 0.4 \\
\hline $\begin{array}{l}0.00 \\
0.02\end{array}$ & $\begin{array}{r}0.000 \\
10.622\end{array}$ & $\begin{array}{r}0.000 \\
16.282\end{array}$ \\
\hline 0.04 & 10.225 & 15.964 \\
\hline 0.06 & 9.870 & 15.647 \\
\hline 0.08 & 9.546 & 15.329 \\
\hline 0.20 & 9.242 & 15.012 \\
\hline 0.12 & 8.949 & 14.695 \\
\hline 0.24 & 8.659 & 14.378 \\
\hline 0.16 & 8.362 & 14.062 \\
\hline 0.18 & 8.054 & 13.747 \\
\hline 0.20 & 9.301 & 13.432 \\
\hline 0.22 & 9.394 & 13.118 \\
\hline 0.24 & 9.397 & 12.804 \\
\hline 0.26 & 9.385 & 12.332 \\
\hline 0.28 & 9.402 & 14.857 \\
\hline 0.30 & 9.393 & 14.819 \\
\hline 0.32 & 9.389 & 14.910 \\
\hline 0.34 & 9.380 & 14.986 \\
\hline 0.36 & 9.362 & 15.060 \\
\hline 0.38 & 9.345 & 25.145 \\
\hline 0.40 & 9.332 & 15.244 \\
\hline 0.42 & 9.320 & 15.359 \\
\hline 0.44 & 9.311 & 15.490 \\
\hline $\begin{array}{l}0.46 \\
0.48\end{array}$ & $\begin{array}{l}9.304 \\
9.298\end{array}$ & $\begin{array}{l}15.635 \\
15.793\end{array}$ \\
\hline $\begin{array}{l}0.48 \\
0.50\end{array}$ & $\begin{array}{l}9.298 \\
9.292\end{array}$ & $\begin{array}{l}15.793 \\
15.961\end{array}$ \\
\hline 0.52 & 9.286 & 16.137 \\
\hline 0.54 & 9.280 & 16.318 \\
\hline 0.56 & 9.274 & 16.501 \\
\hline 0.58 & 9.267 & 16.682 \\
\hline 0.60 & 9.259 & .859 \\
\hline 0.62 & 9.250 & .032 \\
\hline 0.64 & 9.240 & 17.196 \\
\hline 0.66 & 9.229 & 17.350 \\
\hline 0.68 & 9.218 & 17.492 \\
\hline 0.70 & 9.206 & 17.619 \\
\hline 0.72 & $\begin{array}{l}9.194 \\
9.181\end{array}$ & 17.732 \\
\hline 0.74 & $\begin{array}{l}9.181 \\
9.167\end{array}$ & 17.830 \\
\hline 0.76 & $\begin{array}{l}9.167 \\
9.152\end{array}$ & 17.911 \\
\hline 0.78 & $\begin{array}{l}9.1 .52 \\
9.137\end{array}$ & 976 \\
\hline 0.80 & $\begin{array}{l}9.137 \\
9.120\end{array}$ & .026 \\
\hline $\begin{array}{l}0.82 \\
0.84\end{array}$ & $\begin{array}{l}9.120 \\
9.104\end{array}$ & 18.062 \\
\hline $\begin{array}{l}0.84 \\
0.86\end{array}$ & $\begin{array}{l}9.104 \\
9.087\end{array}$ & 18.083 \\
\hline $\begin{array}{l}0.86 \\
0.88\end{array}$ & $\begin{array}{l}9.087 \\
9.069\end{array}$ & $\begin{array}{l}18.091 \\
18.088\end{array}$ \\
\hline 0.90 & 9.051 & 18.075 \\
\hline 0.92 & 9.033 & 18.054 \\
\hline 0.94 & 9.015 & 18.027 \\
\hline 0.96 & 8.997 & 17.995 \\
\hline 0.98 & 979 & 17.960 \\
\hline & 000 & 200 \\
\hline
\end{tabular}

Homologous Parameter

$\begin{array}{ll}0.6 & 0.8\end{array}$

0.000

0.000

1.0

1.2

1.4

24.525

24.045

32.846

32.201

23.566

23.087

22.609

22.131

21.654

21.177

20.700

19.981

19.098

18.246

17.333

16.691

16.272

17.481

17.201

16.815

16.944

16.834

17.019

17.400

18.414

18.881

19.307

19.753

20.234

20.752

21.304

21.882

22.477

23.077

23.670

24.243

24.784

25.283

31.557

30.914

30.271

29.629

28.987

28.346

27.705

27.066

26.427

25.789

25.151

24.515

23.879

23.250

22.369

21.326

20.454

19.624

20.336

19.848

19.514

19.460

19.721

20.274

21.625

22.717

23.649

24.645

25.725

26.880

28.089

29.325

30.554

31.740

32.846

26.124

33.842

26.456

34.703

35.412

26.726

26.935

27.086

27.183

27.231

27.237

27.208

27.151

27.075

26.986

0.000

35.964

36.360

36.612

36.733

36.745

36.667

36.520

36.324

36.099

0.000
0.000

41.285

40.469

39.655

38.841

38.028

37.216

36.405

35.596

34.787

33.979

33.173

32.368

31.564

30.761

29.960

29.163

28.369

27.575

26.785

25.999

25.218

24.445

23.688

22.962

22.557

22.121

22.072

22.575

23.650

25.853

27.414

29.084

30.920

32.908

35.003

37.139

39.234

41.199

42.947

44.406

45.533

46.316

46.775

46.950

46.891

46.652

46.285

45.835

45.342

0.000
0.000

49.900

48.901

47.905

46.910

45.918

44.928

43.939

42.953

41.969

40.987

40.007

39.028

.38 .052

37.077

36.105

35.135

34.174

33.212

32.252

31.297

30.347

29.402

28.465

27.540

26.633

25.762

25.248

24.696

24.619

25.399

27.250

30.171

32.571

35.268

38.274

41.534

44.941

48.336

51.514

54.247

56.346

57.719

58.394

58.483

58.132

57.481

56.646

55.715

54.758

0.000
0.000

58.788

57.583

56.384

55.190

54.001

52.816

51.636

50.460

49.288

48.120

46.955

45.794

44.636

43.481

42.330

41.183

40.047

38.909

37.776

36.648

35.524

34.407

33.297

32.197

31.109

30.039

28.998

28.014

27.519

27.056

27.541

29.484

33.345

36.668

40.516

44.946

49.922

55.323

60.875

66.064

70.158

72.563

73.247

72.668

66.327

67.038

67.678

68.263

68.807

0.000 
Inlet Void

Fraction

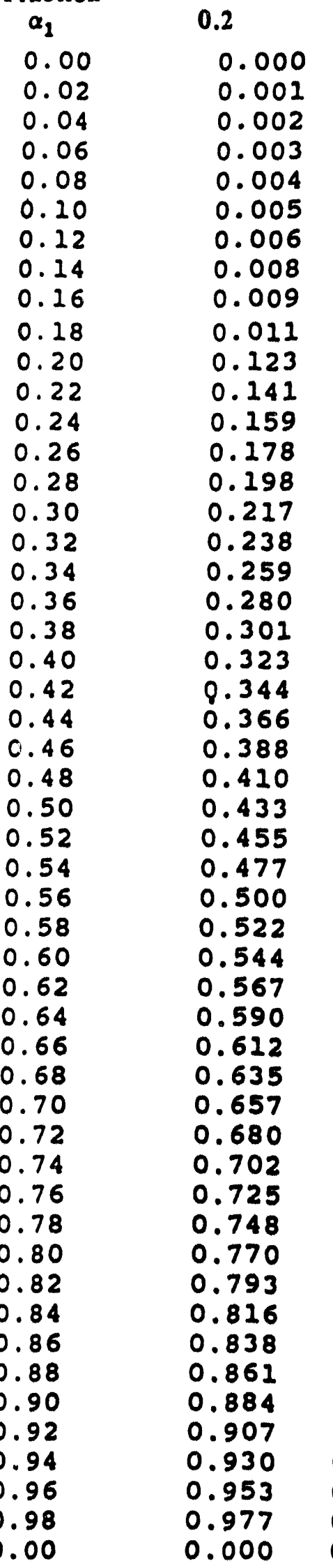

$\alpha_{2}$ Void Fraction

Homologous Parameter

$\begin{array}{llllll}0.4 & 0.6 & 0.8 & 1.0 & 1.2 & 1.4 \\ 0.000 & 0.000 & 0.000 & 0.000 & 0.000 & 0.000 \\ 0.001 & 0.001 & 0.001 & 0.001 & 0.001 & 0.001 \\ 0.002 & 0.002 & 0.002 & 0.002 & 0.003 & 0.003 \\ 0.003 & 0.003 & 0.003 & 0.004 & 0.004 & 0.004 \\ 0.004 & 0.004 & 0.005 & 0.005 & 0.005 & 0.005 \\ 0.005 & 0.006 & 0.006 & 0.006 & 0.007 & 0.007 \\ 0.006 & 0.007 & 0.007 & 0.008 & 0.008 & 0.008 \\ 0.008 & 0.008 & 0.009 & 0.009 & 0.009 & 0.010 \\ 0.009 & 0.010 & 0.010 & 0.010 & 0.011 & 0.011 \\ 0.011 & 0.011 & 0.012 & 0.012 & 0.013 & 0.013 \\ 0.012 & 0.013 & 0.013 & 0.014 & 0.014 & 0.015 \\ 0.014 & 0.015 & 0.015 & 0.015 & 0.016 & 0.016 \\ 0.016 & 0.017 & 0.017 & 0.017 & 0.018 & 0.018 \\ 0.018 & 0.019 & 0.018 & 0.019 & 0.01020 & 0.020 \\ 0.098 & 0.022 & 0.020 & 0.021 & 0.022 & 0.022 \\ 0.099 & 0.024 & 0.022 & 0.023 & 0.024 & 0.024 \\ 0.118 & 0.032 & 0.025 & 0.025 & 0.026 & 0.027 \\ 0.139 & 0.047 & 0.028 & 0.028 & 0.029 & 0.029 \\ 0.162 & 0.055 & 0.031 & 0.031 & 0.031 & 0.032 \\ 0.186 & 0.084 & 0.035 & 0.033 & 0.034 & 0.034 \\ 0.212 & 0.098 & 0.039 & 0.037 & 0.037 & 0.037 \\ 0.239 & 0.123 & 0.049 & 0.040 & 0.040 & 0.041 \\ 0.268 & 0.150 & 0.059 & 0.044 & 0.044 & 0.044 \\ 0.297 & 0.176 & 0.076 & 0.049 & 0.048 & 0.048 \\ 0.326 & 0.208 & 0.103 & 0.056 & 0.053 & 0.053 \\ 0.357 & 0.244 & 0.139 & 0.074 & 0.059 & 0.058 \\ 0.387 & 0.282 & 0.179 & 0.092 & 0.066 & 0.064 \\ 0.417 & 0.321 & 0.217 & 0.123 & 0.085 & 0.071 \\ 0.447 & 0.361 & 0.260 & 0.169 & 0.104 & 0.080 \\ 0.477 & 0.401 & 0.308 & 0.222 & 0.137 & 0.104 \\ 0.506 & 0.441 & 0.358 & 0.269 & 0.191 & 0.129 \\ 0.535 & 0.480 & 0.408 & 0.327 & 0.254 & 0.178 \\ 0.563 & 0.518 & 0.458 & 0.388 & 0.313 & 0.249 \\ 0.590 & 0.554 & 0.505 & 0.447 & 0.383 & 0.316 \\ 0.617 & 0.588 & 0.550 & 0.505 & 0.453 & 0.396 \\ 0.643 & 0.621 & 0.593 & 0.558 & 0.519 & 0.475 \\ 0.669 & 0.653 & 0.632 & 0.608 & 0.580 & 0.549 \\ 0.694 & 0.683 & 0.668 & 0.653 & 0.636 & 0.617 \\ 0.719 & 0.711 & 0.702 & 0.693 & 0.684 & 0.676 \\ 0.743 & 0.738 & 0.733 & 0.729 & 0.727 & 0.727 \\ 0.767 & 0.764 & 0.762 & 0.761 & 0.763 & 0.769 \\ 0.791 & 0.789 & 0.789 & 0.790 & 0.794 & 0.804 \\ 0.814 & 0.814 & 0.814 & 0.816 & 0.821 & 0.831 \\ 0.838 & 0.837 & 0.838 & 0.841 & 0.846 & 0.854 \\ 0.861 & 0.861 & 0.862 & 0.864 & 0.868 & 0.875 \\ 0.884 & 0.884 & 0.885 & 0.887 & 0.890 & 0.936 \\ 0.907 & 0.907 & 0.908 & 0.909 & 0.911 & 0.949 \\ 0.930 & 0.930 & 0.931 & 0.931 & 0.933 & 0.962 \\ 0.953 & 0.953 & 0.954 & 0.954 & 0.954 & 0.975 \\ 0.977 & 0.977 & 0.977 & 0.977 & 0.977 & 0.987 \\ 0.000 & 0.000 & 0.000 & 0.000 & 0.000 & 0.000\end{array}$


Table 3.5.2-1d $\quad\left(p_{1}=\quad \mathrm{atm}\right)$

Static Pressure Increase, $\left(p_{2}-p_{1}\right)(p s l)$

Inlet Void

\section{Fraction}

$\alpha_{1}$

0.00

0.02

0.04

0.06

0.08

0.10

0.12

0.14

0.16

0.18

0.20

0.22

0.24

0.26

0.28

0.30

0.32

0.34

0.36

0.38

0.40

0.42

0.44

0.46

0.48

0.50

0.52

0.54

0.56

0.58

0.60

0.62

0.64

0.66

0.68

0.70

0.72

0.74

0.76

0.78

0.80

0.82

0.84

0.86

0.88

0.90

0.92

0.94

0.96

0.98

0.00
0.2

259.688

259.217

258.733

258.203

257.602

256.901

256.072

255.086

253.915

252.530

6.754

5.647

5.015

4.478

4.037

3.632

3.279

2.978

2.708

2.462

2.237

2.031

1.844

1.671

1.514

1.371

1.240

1.121

1.013

0.914

0.825

0.743

0.669

0.602

0.542

0.487

0.438

0.394

0.353

0.318

0.285

0.255

0.228

0.204

0.181

0.162

0.141

0.123

0.105

0.089

0.000
0.4

250.569

250.607

250.490

250.316

250.043

249.805

249.451

249.027

248.527

247.940

247.261

246.465

245.517

244.488

42.984

35.735

29.791

25.069

21.258

18.104

15.463

13.235

11.343

9.727

8. 342

7.152

6.124

5.237

4.470

3.807

3.233

2.736

2.308

1.939

1.623

1.351

1.121

0.925

0.761

0.623

0.509

0.415

0.339

0.277

0.226

0.187

0.155

0.130

0.108

0.091

0.000

Homologous Parameter$$
0.6
$$

0.8

1.0

1.2

1.4

$\begin{array}{llllll}238.569 & 220.437 & 192.918 & 152.761 & 96.712\end{array}$

$\begin{array}{llllll}239.102 & 221.645 & 194 \% .422 & 154.519 & 98.253\end{array}$

$\begin{array}{llllll}239.590 & 222.668 & 195.894 & 156.234 & 99.759\end{array}$

$\begin{array}{llllll}240.038 & 223.652 & 197.331 & 157.915 & 101.240\end{array}$

$\begin{array}{lllllll}240.443 & 224.594 & 198.727 & 159.561 & 102.696\end{array}$

$240.803 \quad 225.494200 .082 \quad 161.170 \quad 104.125$

$\begin{array}{llllll}241.113 & 226.347 & 201.396 & 162.740 & 106.015\end{array}$

$241.371227 .148 \quad 202.660 \quad 164.271 \quad 107.464$

$\begin{array}{lllllll}241.571 & 227.898 & 203.878 & 165.757 & 108.841\end{array}$

$\begin{array}{llllll}241.709 & 228.597 & 205.042 & 167.228 & 109.815\end{array}$

$\begin{array}{llllll}241.780 & 229.239 & 206.3 .50 & 169.350 & 110.522\end{array}$

$\begin{array}{llllll}241.778 & 229.820 & 207.199 & 169.888 & 111.049\end{array}$

$241.695230 .330 \quad 208.185120 .463 \quad 110.940$

$\begin{array}{llllll}241.521 & 230.765 & 209.110 & 171.184 & 112.459\end{array}$

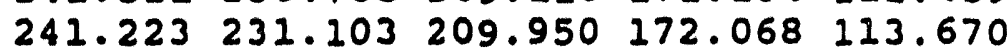

$240.713 \quad 231.316 \quad 210.684 \quad 174.649 \quad 114.808$

$\begin{array}{llllll}236.811 & 230.254 & 211.240 & 175.568 & 116.149\end{array}$

$234.112229 .326 \quad 210.375 \quad 175.308 \quad 117.488$

$229.801 \quad 228.447210 .310 \quad 175.702 \quad 118.000$

$124.442 \quad 227.223 \quad 210.078 \quad 176.017 \quad 118.523$

$97.312 \quad 225.493 \quad 209.577 \quad 176.154 \quad 118.931$

$\begin{array}{llllll}73.510 & 223.007 & 208.753 & 176.079 & 119.210\end{array}$

$\begin{array}{llllll}55.896 & 219.262 & 207.500 & 175.741 & 119.335\end{array}$

$43.116 \quad 212.671205 .696 \quad 175.089 \quad 119.277$

$\begin{array}{llllll}33.736 & 147.068 & 203.088 & 174.035 & 119.005\end{array}$

$26.720 \quad 112.582 \quad 199.228 \quad 172.461 \quad 118.462$

$\begin{array}{llllll}21.353 & 78.404 & 192.985 & 170.174 & 117.580\end{array}$

17.168

13.854

11.198

9.050

7.299

5.868

4.694

54.760

178.3

$166.826 \quad 116.257$

39.397

121.057

161.693

114.313

29.089

81.14

21.829

52.25

16.543

35.183

152.630

111.461

12.592

24.529

76.755

107.050

3.730

9.584

17.432

45.828

99.193

2.938

7.264

12.487

28.911

70.557

2.292

5.457

8.927

19.042

41.765

1.766

4.043

6.307

12.788

23.872

1.342

2. 937

4.349

8.587

14.606

1.004

2.075

2.874

5.649

9.200

0.738

1. 411

1.764

3.526

5.766

0.533

0.911

0.944

1.955

3.440

0.543

0.362

0.378

0.286

$-0.017$

0.767

1.755

0.264

0.117

$-0.232$

$-0.125$

0.353

0.184

0.016

$-0.322$

$-0.728$

$-0.814$

0.132

$-0.033$

$-0.329$

$-0.977$

$-2.775$

0.099

$-0.045$

0.081

0.073

0.072

$-0.032$

$-0.282$

$-0.93$

$-1.999$ 
$v_{12}$ Liqui 1 Flow Velocity (feet/sec)

Inlet Void

Fraction

$$
a_{1}
$$

0.00

0.02

0.04

0.06

0.08

0.10

0.12

0.14

0.16

0.18

0.20

0.22

0.24

0.26

0.28

0.30

0.32

0.34

0.36

0.38

0.40

0.42

0.44

0.46

0.48

0.50

0.52

0.54

0.56

0.58

0.60

0.62

0.64

0.66

0.68

0.70

0.72

0.74

0.76

0.78

0.80

0.82

0.84

0.86

0.88

0.90

0.92

0.94

0.96

0.98

0.00
0.2

9.078

8.900

8.722

8.545

8.367

8.189

8.012

7.835

7.657

7.480

8.029

7.985

7.931

7.872

7.840

7.797

7.765

7.741

7.717

7.700

7.689

7.682

7.679

7.678

7.680

7.684

7.689

7.696

7.704

7.712

7.721

7.731

7.742

7.752

7.761

7.769

7.777

7.783

7.788

7.791

7.793

7.794

7.794

7.793

7.791

7.789

7.787

7.785

7.783

7.782

0.000
0.4

18.155

17.801

17.446

17.091

16.744

16.379

16.023

15.668

15.313

14.958

14.604

14.250

13.906

13.547

16.913

13.756

13.390

13.104

12.934

12.797

12.692

12.620

12.579

12.571

12.594

12.648

12.731

12.843

12.979

13.138

13.316

13.509

13.713

13.92 ?

14.131

14.334

14.528

14.708

14.873

15.019

15.146

15.252

15.339

15.407

15.458

15.494

25.518

15.533

15.541

15.546

0.000

\section{Homologous Parameter}

0.6

27.233

0.8

26.704

26.173

25.643

25.112

24.581

24.050

23.519

22.988

22.458

21.927

21.396

20.865

20.335

19.804

19.274

18.746

18.211

17.652

17.497

17.295

17.100

16.815

16.267

16.038

15.926

15.894

15.942

16.071

16.286

16.585

16.968

17.430

17.960

18.544

19.164

19.800

20.429

21.030

21.583

22.072

22.485

22.818

23.070

23.246

23.355

23.407

23.415

23.391

23.349

0.000

36.311

35.607

34.900

34.193

33.487

32.780

32.073

31.366

30.659

29.951

29.244

28.537

27.829

27.122

26.415

25.707

25.002

24.296

23.590

22.886

22.182

21.480

20.779

20.069

19.628

18.818

18.849

18.658

18.626

18.563

18.754

19.114

19.650

20.367

21.263

22.326

23.528

24.829

26.171

27.484

28.696

29.741

30.573

31.173

31.549

31.728

31.747

31.649

31.473

31.256

0.000

1.0
45.389

44.510

43.628

42.746

41.854

40.981

40.099

39.216

38.332

37.449

36.565

35.681

34.797

33.913 .

33.029

32.145

31.261

30.379

29.497

28.614

27.733

26.852

25.973

25.095 
va Gas Flow Velocity (feet/sec)

Inlet Vold
Fraction

$\alpha_{1}$

0.00

0.02

0.04

0.06

0.08

0.10

0.12

0.24

0.16

0.18

0.20

0.22

0.24

0.26

0.28

0.30

0.32

0.34

0.36

0.38

0.40

0.42

0.44

0.46

0.48

0.50

0.52

0.54

0.56

0.58

0.60

0.62

0.64

0.66

0.68

0.70

0.72

0.74

0.76

0.78

0.80

0.82

0.84

0.86

0.88

0.90

0.92

0.94

0.96

0.98

0.00
0.2

0.000

11.298

10.879

10.544

10.269

10.038

9.834

9.645

9.456

9.255

8.769

8.841

8.907

8.971

8.979

8.964

8.960

8.960

8.954

8.938

8.923

8.913

8.908

8.908

8.911

8.917

8.924

8.934

8.944

8.954

8.964

8.973

8.981

8.987

8.994

8.999

9.004

9.007

9.009

9.009

9.009

9.007

9.004

8.999

8.994

8.988

8.983

8.977

8.971

8.966

0.000

Homologous Parameter

0.4

0.000

16.257

25.930

15.605

15.300

14.948

14.621

14.295

13.970

13.646

13.322

13.001

12.690

12.358

16.517

14.179

14.085

13.972

13.959

13.964

13.980

13.994

14.030

14.093

14.184

14.307

14.458

14.636

14.839

15.063

15.303

15.554

15.811

16.070

16.326

16.573

16.808

17.025

17.221

17.393

17.541

17.664

17.762

17.836

17.889

17.923

17.941

17.947

17.943

17.935

0.000
0.6

0.000

24.505

24.012

23.520

23.028

22.537

22.046

21.555

21.064

20.574

20.084

19.594

$19 . .104$

18.614

18.125

17.637

17.150

16.658

16.143

16.024

15.960

15.914

15.811

15.647

16.516

16.846

17.101

17.382

17.719

18.127

18.612

19.175

19.810

20.513

21.269

22.060

22.861

23.647

24.391

25.070

25.663

26.159

26.550

26.838

27.029

27.133

27.166

27.142

27.078

26.990

0.000
0.8

0.000

32.825

32.162

31.499

30.837

30.175

29.513

28.853

28.192

27.533

26.875

26.217

25.560

24.903

24.247

23.591

22.937

22.284

21.631

20.979

20.329

19.680

19.034

18.378

17.890

17.171

17.451

17.578

18.179

19.482

20.152

20.893

21.776

22.832

24.069

25.481

27.044

28.711

30.415

32.070

33.584

34.872

35.875

36.570

36.971

37.118

37.062

36.861

36.564

36.219

0.000

1.0

0.000

41.260

40.419

39.579

38.740

37.903

37.067

36.232

35.397

34.564

33.732

32.901

32.071

31.243

30.416

29.589

28.764

27.942

27.120

26.299

25.479

24.662

23.846 
Inlet Void

Fraction

$$
a_{1}
$$

0.00

0.02

0.04

0.06

0.08

0.10

0.12

0.14

0.16

0.18

0.20

0.22

0.24

0.26

0.28

0.30

0.32

0.34

0.36

0.38

0.40

0.42

0.44

0.46

0.48

0.50

0.52

0.54

0.56

0.58

0.60

0.62

0.64

0.66

0.68

0.70

0.72

0.74

0.76

0.78

0.80

0.82

0.84

0.86

0.88

0.90

0.92

0.94

0.96

0.98

0.00
0.2

$$
0.000
$$

0.000

0.001

0.001

0.002

0.002

0.003

0.003

0.004

0.005

0.093

0.121

0.128

0.145

0.163

0.183

0.203

0.224

0.245

0.267

0.290

0.313

0.336

0.360

0.384

0.408

0.432

0.456

0.480

0.505

0.529

0.553

0.577

0.601

0.625

0.649

0.673

0.696

0.720

0.743

0.767

0.790

0.813

0.837

0.860

0.883

0.907

0.930

0.953

0.977

0.000

$\alpha_{2}$ Void Fraction

Homologous Parameter

0.4

0.000

0.000

0.001

0.001

0.002

0.002

0.003

0.003

0.004

0.005

0.006

0.007

0.008

0.008

0.342

0.092

0.077

0.083

0.099

0.118

0.140

0.164

0.190

0.219

0.249

0.281

0.315

0.349

0.384

0.419

0.454

0.489

0.523

0.556

0.589

0.620

0.650

0.679

0.707

0.734

0.760

0.786

0.811

0.835

0.859

0.883

0.906

0.930

0.953

0.977

0.000
0.6

0.000

0.001

0.001

0.002

0.002

0.003

0.003

0.004

0.005

0.006

0.006

0.007

0.008

0.009

0.010

0.011

0.012

0.013

0.015

0.057

0.060

0.073

0.095

0.106

0.117

0.145

0.177

0.214

0.254

0.297

0.343

0.390

0.437

0.484

0.530

0.574

0.615

0.653

0.689

0.722

0.753

0.782

0.809

0.835

0.859

0.883

0.907

0.930

0.953

0.977

0.000

\section{8}

0.000

0.001

0.001

0.002

0.002

0.003

0.004

0.004

0.005

0.006

0.007

0.007

0.008

0.009

0.010

0.011

0.012

0.014

0.015

0.016

0.018

0.020

0.021

0.024

0.035

0.042

0.082

0.108

0.143

0.179

0.226

0.278

0.335

0.394

0.454

0.512

0.568

0.620

0.667

0.709

0.747

0.780

0.810

0.837

0.862

0.886

0.909

0.931

0.954

0.977

0.000

1.0

0.000

0.001

0.001

0.002

0.002

0.003

0.004

0.005

0.005

0.006

0.007

0.008

0.009

0.010

0.011

0.012

0.013

0.014

0.015 
4.

Air/Water Two-Phase Flow Performance of SRPR (Savannah River Plant Reactor) Pump Under DC Motor Operation (Task 7, Task 9 and Task 16)

\section{$4.1 \quad$ Objectives}

Under the hypothetical loss of coolant accident (LOCA) conditions, there is a possibility that the water level of the coolant tank becomes so low that air may be ingested into the water line. The coolant pump then experiences the air/water flow going through the blades. As has been demonstrated by experimerts conducted by Babcock \& Wilcox (1977) and Creare (1982) as well as the theory of Furuya (1985), the head of the pump operating under the air/water two-phase flow condition severely degrades as the inlet void fraction reaches about $20 \%$ or higher (although this threshold point for the degradation varies depending on the type of pump and operational conditions). It is crucial from the reactor safety viewpoints to determine the extent of the pump head degradation either theoretically or experimentally. However, this type of experiments is too costly and time consuming.

During the normal cooling operation, the SRPR pump is driven by the AC motor at $1,000 \mathrm{rpm}$ as the rated speed, while under the emergency condition, it is operated by the DC motor at $300 \mathrm{rpm}$ as its rated speed. The objective of Task 3, Task 9 and Task 15 was therefore to theoretically determine the head and torque degradation of the SRPR pump operating under the air/water two-phase flow conditions for various physical, hydrodynamic and mechanical conditions at the AC motor operation mode. The parameters which represented such conditions included the inlet void fraction, static pressure at upstream, flow rate and pump rotational speed.

In Tasks 7, 9 and 16, the pump characteristics under the DC motor operation were investigated. Two types of pump flow configuration were used for the present work as before. One is that normally used for the single phase pump design and analysis where the pump blade flow passage is used with the diffuser effect being ignored or treated empirically in terms of the head loss. (Task 7 and Task 9) The other is that with the diffuser section incorporated. (Task 16) It should be also noted that the H-Q curve used for the former (Task 7 and Task 9) is different from that of the latter. The reason is that the results of curve fitting for the single phase pump performance based on the experimental data changed from time to time. When the two-phase flow pump performance without diffuser was calculated some time ago, only the $\mathrm{H}-\mathrm{Q}$ curve available was the one obtained at an earlier stage of the experiments which was thus used then. On the other hand, the calculation with the diffuser 
section incorporated was made with the $H-Q$ curve obtained form more recent experiments.

4.2 Theoretical Basis---Without Diffuser (Task 7 and Task 9)

The theoretical basis, the definition of pump head under the two-phase flow condition and numerical solution method for calculating the performance of the SRPR pump without the diffuser section remain the same as those for the AC motor operations. The reader is therefore advised to refer to Section 3.2 of this report.

Nomenclature

\begin{tabular}{|c|c|}
\hline A: & cross sectional area normal to the direction of $s$ \\
\hline$C_{d}:$ & drag coefficient \\
\hline $\mathrm{C}_{\mathrm{vm}}:$ & virtual (or added) mass coefficient \\
\hline$d s:$ & infinitesimal increment in the s-coordinate \\
\hline g: & gravitational acceleration \\
\hline h: & normalized head $\left(=\mathrm{H} / \mathrm{H}_{\mathrm{R}}\right)$ \\
\hline H: & head in feet \\
\hline m: & mass flow rate \\
\hline $\mathbf{N}:$ & pump rotational speed (rpm) \\
\hline p: & static pressure \\
\hline Q: & volumetric flow rate \\
\hline r: & radial distance measured from the center of pump rotation \\
\hline $\mathbf{r}_{\mathbf{s}}:$ & radius of gas bubble \\
\hline s: & streamline coordinate \\
\hline v: & absolute velocity \\
\hline w: & flow velocity relative to the rotating blade in the s-direction \\
\hline $\mathbf{x :}$ & gas mass flow rate ratio $\left(=m_{\mathbf{c}} / \mathbf{m}\right)$ \\
\hline$\alpha$ & void fraction \\
\hline$\alpha_{N}:$ & normalized rotational speed $\left(=\mathrm{N} / \mathrm{N}_{\mathbf{R}}\right)$ \\
\hline$\nu$ & normalized flow rate $\left(=Q / Q_{R}\right)$ \\
\hline p: & density \\
\hline$\omega$. & angular velocity of pump rotor $(=2 \pi N / 60)$ \\
\hline
\end{tabular}


Subscripts:

8,l: denotes the quantities related to the gas and liquid phases, respectively

1,2: demotes the quantities related to the inlet and exit of the pump, respectively

R: $\quad$ denotes the quantities at the pump rated condition

2ф: $\quad$ two-phase flow quantities

1ф: $\quad$ single-phase flow quantities

4.3 Numerical Calculations---Without Diffuser (Task 7)

4.3.1 Parametric Range

1) Pump

The SRPR pump is a double suction, double-volute centrifugal pump manufactured by Bingham Corp. having the rated flow rate of $25,000 \mathrm{gpm}(12,500 \mathrm{gpm} /$ volute) with the total head of 420 feet at the rated rotational speed of 1,000 rpm under the AC motor operation. On the other hand, when this pump is operated by the DC motor at the rotation speed of $300 \mathrm{rpm}$, the flow rate is $6,200 \mathrm{gpm}$ (3,100 gpm/volute).

The head vs. flow rate curve driven by the AC motor at 1,000 rpm and by the DC motor at $300 \mathrm{rpm}$ are given in Figures 4.3.1-1 and 4.3.1-2, respectively. In these figures both the total pump dynamic head and that of fluid flowing are given for the AC motor pump performance while only the latter is given for the DC motor case. The correction is thus to be made for the DC motor head curve as follows:

$$
\begin{gathered}
H_{1 \phi D C}=47.7-0.4289 \times(Q / 1000)^{0.943}+\Delta H \\
\Delta H=4.236 \times(Q / 10,000)^{2}
\end{gathered}
$$

where the formula for Equations (4.3.1-1) and (4.3.1-2) were given by the report No. DPS-83-282 and the Los Alamos National Laboratory. Therefore, the rated head at the DC motor operation will be readily calculated 


$$
H_{1 \phi D C \text { at } 300 \mathrm{rpm}}=46.93 \text { feet. }
$$

It should be noted that the homologous flow parameters and head data precented hereafter for the DC motor operation are all normalized by using $N_{R s 00}=300 \mathrm{rpm}, Q_{R s 00}=6200 \mathrm{gpm}$ and $H_{R 300}=46.93$ feet.

The pump performance curves nondimensionalized by using the unified rated parameters (i.e., $N_{R}$, $Q_{R}$ and $H_{R}$ at $1,000 \mathrm{rpm}$ ) show a clear difference between the $A C$ and $D C$ motor operations(see Figure 4.3.1-3)).

2) Line Static Pressure $\left(p_{1}\right)$

The following line static pressures were used for the present calculations:

$$
\begin{aligned}
& 0.05 \mathrm{~atm} \\
& 1.00 \mathrm{~atm} \\
& 2.00 \mathrm{~atm} \\
& 3.00 \mathrm{~atm}
\end{aligned}
$$

3) Flow Rate ( Q ) and Pump Rotational Speed (N)

Since the pump performance only depends on the ratio between the flow rate and the rotational speed, the range of these parameters is expressed in terms of the homologous flow parameter defined by $\nu$ $/ \alpha_{N}$ where

$$
\begin{gathered}
\nu=Q / Q_{R} \\
\alpha_{N}=N / N_{R} .
\end{gathered}
$$

The range of the homologous head parameter used for the calculations is

$$
\begin{aligned}
& \nu / \alpha_{N}=0.4 \\
& 0.6 \\
& 0.8 \\
& 1.0
\end{aligned}
$$




\section{2}

1.4

With these homologous parameters, the range of the actual flow rate will be $2,480 \mathrm{gpm}$ to $8,680 \mathrm{gpm}$ assuming the constant rotational speed (i.e., $300 \mathrm{rpm}$ ). It is to be noted however that the present twophase flow pump performance is calculated on the basis of the H-Q curve with the DC motor rated condition used as described above.

4) Inlet Void Fraction $\left(\alpha_{1}\right)$

$$
\alpha_{1}=0.00 \text { to } 0.98
$$

\subsubsection{Results}

Figures 4.3.2-1a to $1 \mathrm{~d}$ show the homologous head as the function of the inlet void fraction with the homologous flow parameter $\nu / \alpha_{N}$ ranging from 0.4 to 1.4 at the static pressure $p_{1}=3$ atm, 2 atm, $1 \mathrm{~atm}$ and $0.05 \mathrm{~atm}$, respectively.

Tables 4.3.2-1a to Id simply provide the homologous head data used for the figures above in terms of the numbers.

Tables 4.3.2-2 to 5 are the detailed pump hydraulic data as discussed in Section 3.2.4 of the report mentioned above (OF-88-003). Tables 4.3.2-2a to $2 \mathrm{~d}$ provide the void fraction at the exit of the pump blade for the same inlet static pressure range while Tables $4.3 .2-3 \mathrm{a}$ to $3 \mathrm{~d}$ present the static pressure at the pump blade exit.

Tables 4.3.2-4a to $4 \mathrm{~d}$ and 4.3.2-5a to $5 \mathrm{~d}$ provide the relative flow velocities of water and air at the blade exit of the pump, respectively. These relative flow velocities in these tables were normalized by the pump blade tip speed $u_{2}$ at the rated pump rotational speed of $300 \mathrm{rpm}$ :

$$
\begin{aligned}
u_{2 \text { at } 300 \mathrm{rpm}} & =2 \pi N_{R 2} / 60 \\
& =2 \pi \times 300 \times 21^{\prime \prime} / 12 / 60 \\
& =\$ 4.98 \mathrm{ft} / \mathrm{sec}
\end{aligned}
$$


The absolute velocities of water and air at the exit of the pump blade will be readily calculated from the velocity triangle with the blade angle at the exit $\beta=15.071^{\circ}$ known (see the table at page $2-2$ of the interim report for Task 2 ).

The homologous torques for these cases were also calculated based on the formula given in Section 3.2.5 and are shown in Tables 4.3.2-1a to $1 \mathrm{~d}$.

The same argument as that for the AC motor operation (see Section 3.4) is applied to the present case of the DC motor operation with the diffuser section incorporated.

4.5 Numerical Calculations---With Diffuser (Task 16)

\subsubsection{Parametric Range}

1) Pump
Rated Flow Rate $Q_{R}: \quad 6,200 \mathrm{gpm}(3,100 \mathrm{gpm} /$ volute)
Rated Head $\mathrm{H}_{\mathbf{R}}$ :
45.3 feet (Experimental; from Equation (4.5.1) below)
Rotational Speed (DC Motor):
$300 \mathrm{rpm}$

where the H-Q curve for the Savannah River Plant reactor pump is obtained from

$\Delta H=C_{0}+C_{1} Q+C_{2} Q^{2}+C_{3} Q^{3}$

where

$$
\begin{aligned}
\Delta H & =\text { pump head }\left(\mathrm{ft} \text { of } D_{2} O\right) \\
Q & =\text { flow rate }(\mathrm{kgpm}) \\
C_{0} & =562.16 \\
C_{1} & =-4.4957 \\
C_{2} & =0.15145 \\
C_{3} & =-0.0090580 .
\end{aligned}
$$


2) Line Static Pressure $\left(p_{1}\right)$

The following line static pressures were used for the present calculations:

$$
\begin{aligned}
& 0.5 \mathrm{~atm} \\
& 1.00 \mathrm{~atm} \\
& 2.00 \mathrm{~atm} \\
& 3.00 \mathrm{~atm}
\end{aligned}
$$

3) Flow Rate ( $Q$ ) and Pump Rotational Speed (N)

Since the pump performance depends on the ratio between the flow rate and the rotational speed, the range of these parameters is expressed in terms of the homologous flow parameter defined by $\nu / \alpha_{N}$ where

$$
\begin{gathered}
\nu=Q / Q_{R} \\
\alpha_{N}=N / N_{R} .
\end{gathered}
$$

The range of the homologous head parameter used for the calculations is

$$
\begin{aligned}
& \nu / \alpha_{N}=0.2 \\
& 0.4 \\
& 0.6 \\
& 0.8 \\
& 1.0 \\
& 1.2 \\
& 1.4
\end{aligned}
$$

With these homologous parameters, the range of the actual flow rate will be $1,240 \mathrm{gpm}$ to $8,680 \mathrm{gpm}$ assuming the constant rotational speed (i.e., $300 \mathrm{rpm}$ ).

4) Inlet Void Fraction $\left(\alpha_{1}\right)$

$$
\begin{array}{r}
\alpha_{1}=0.00 \text { to } 0.98 \\
4-7
\end{array}
$$




\subsubsection{Results}

Tables 4.5.2-1a to Id show the homologous head as the function of the inlet void fraction with the homologous flow parameter $\nu / \alpha_{N}$ ranging from 0.2 to 1.4 at the static pressure $p_{1}=3$ atm, 2 atm, $1 \mathrm{~atm}$ and $0.5 \mathrm{~atm}$, respectively.

4.6 References

OF Technologies, Inc., 1988, Air/water two-phase flow performance of SRPR pump under AC motor operation, Report No. OF-88-003, September. 
Flgure 4.3.1-1

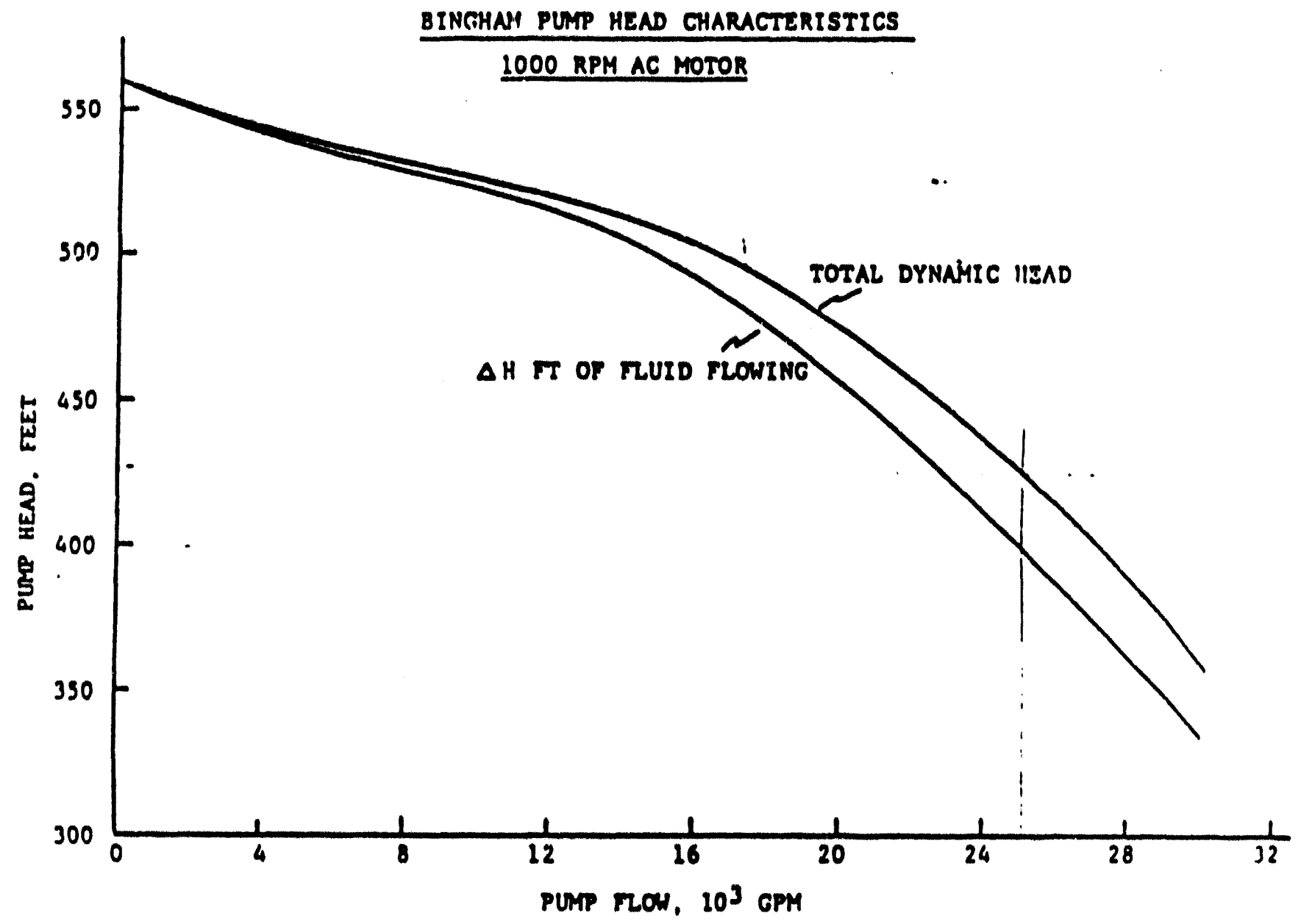

4-9 
Flgure 4.3.1-2

BINGHAM PUMP HEAD CHARACTERISTICS

300 RPM DC MOTOR

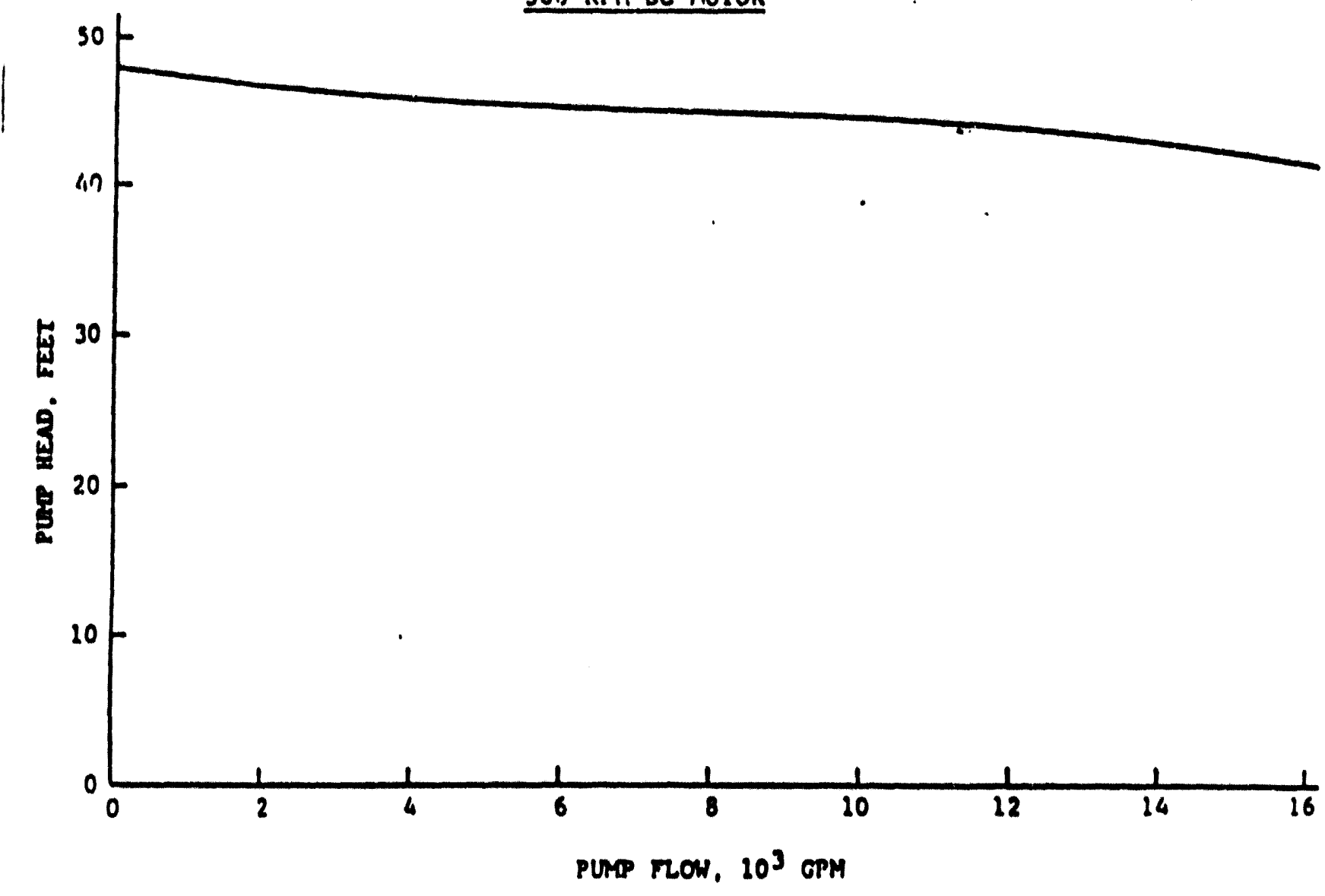

$4 \cdot 10$ 
Figare 4.3.1-3

Single-phase Performance of SRPRP $A C$ and DC Mototr Performances

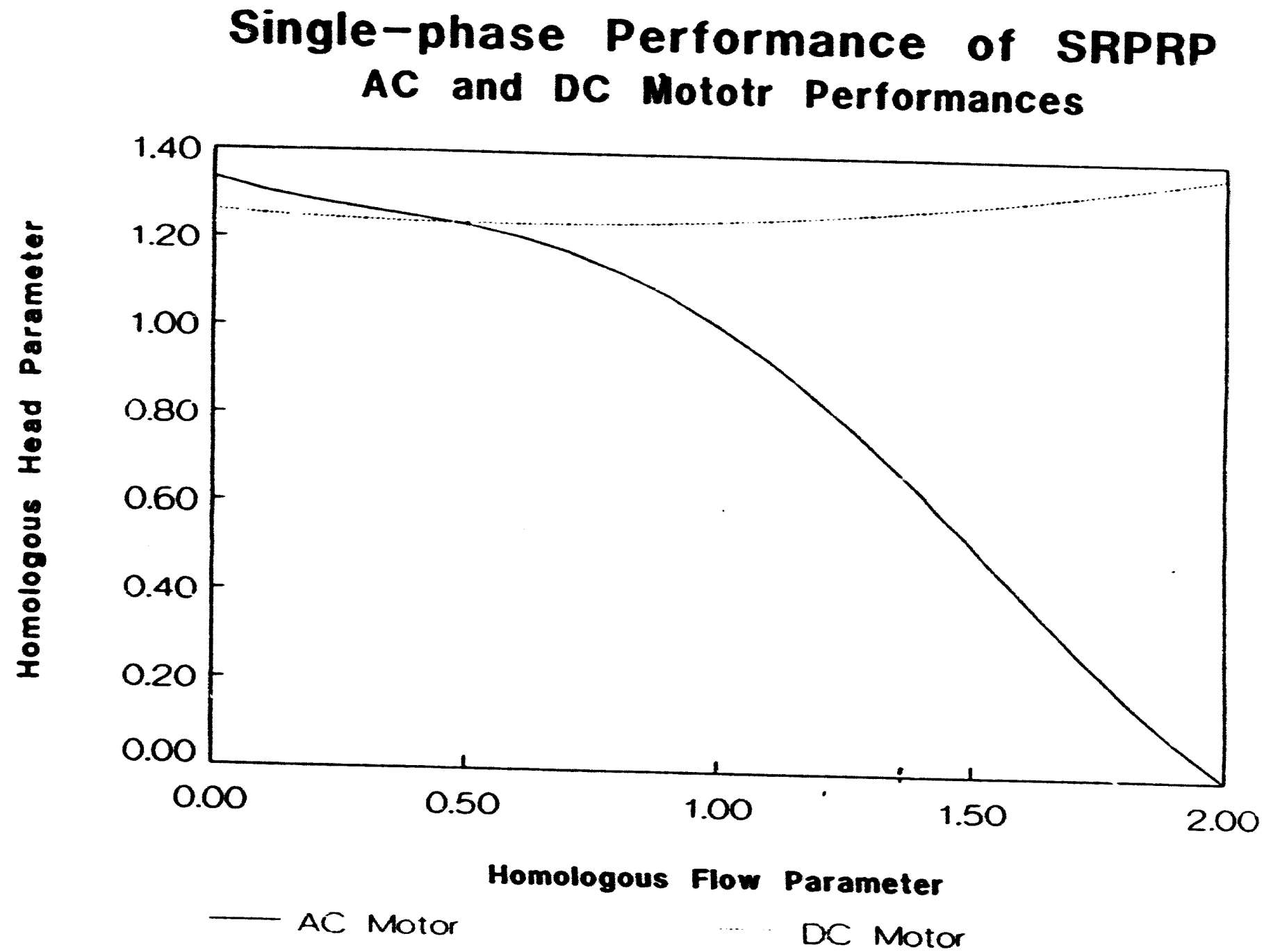


Fleure 4.3.2-1a ( $\left.\quad p_{1}=3 \mathrm{ata}\right)$

Head Degradation of SRPRP

Air-Water Case. DC Motor. P1 = $3 \mathrm{~atm}$

$v / a$

0.6

08

1.0

12

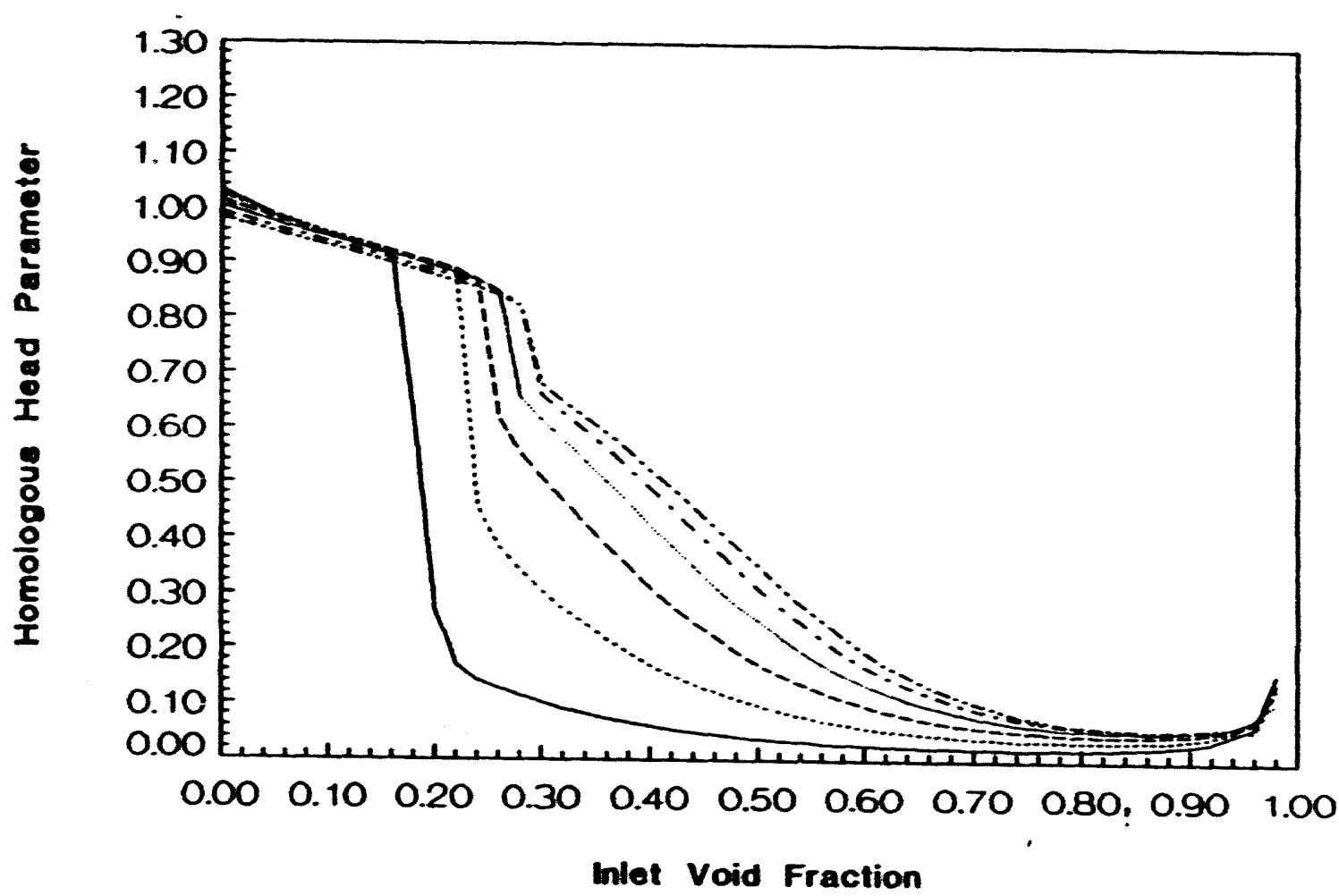


Figure 4.3.2-1b

$\left(p_{1}=2 \mathrm{~atm}\right)$

\section{Head Degradation of SRPRP}

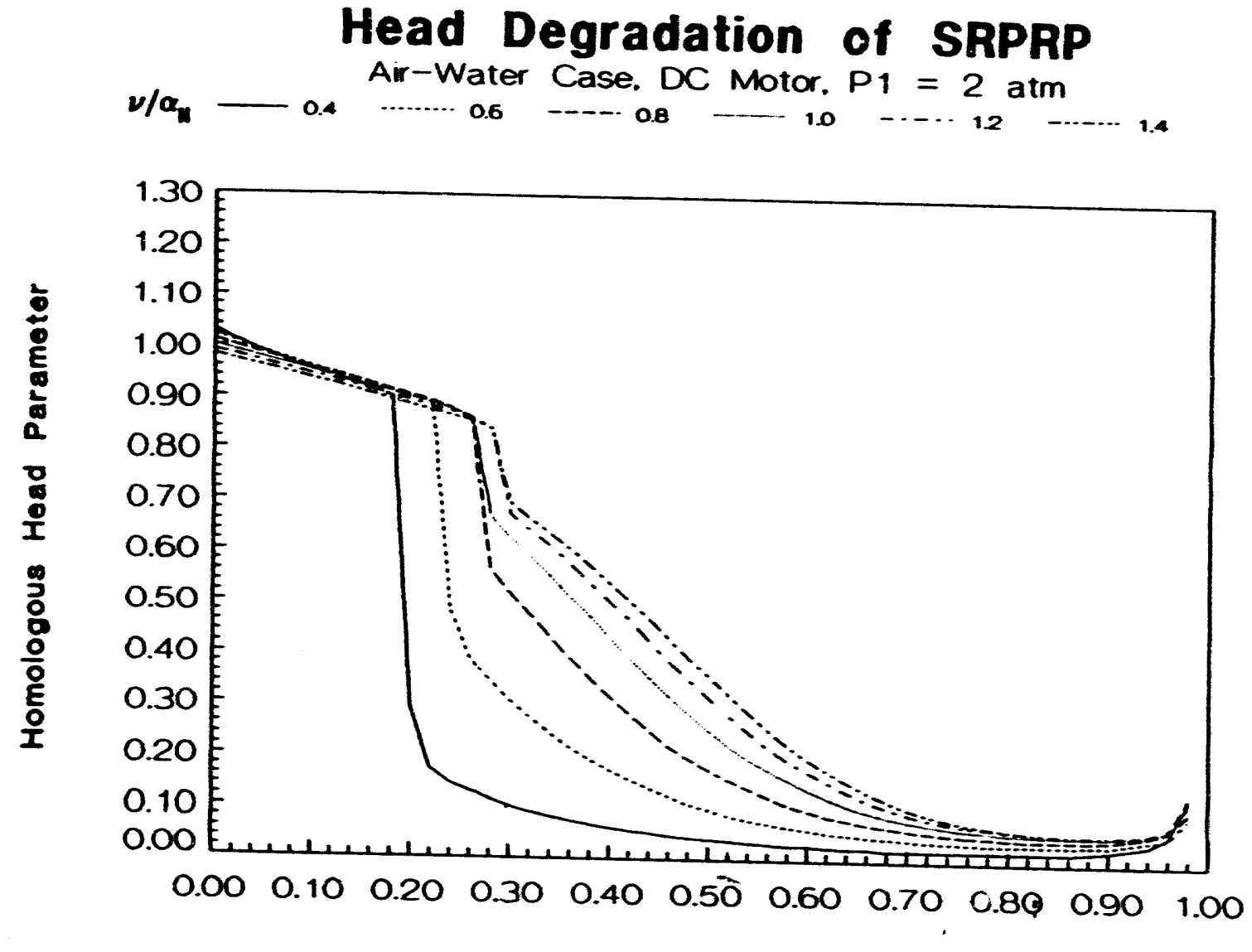

Inlet Void Fraction 
Figure 4.3.2-1c $\quad\left(p_{1}=1 \mathrm{~atm}\right)$

\section{Head Degradation of SRPRP}

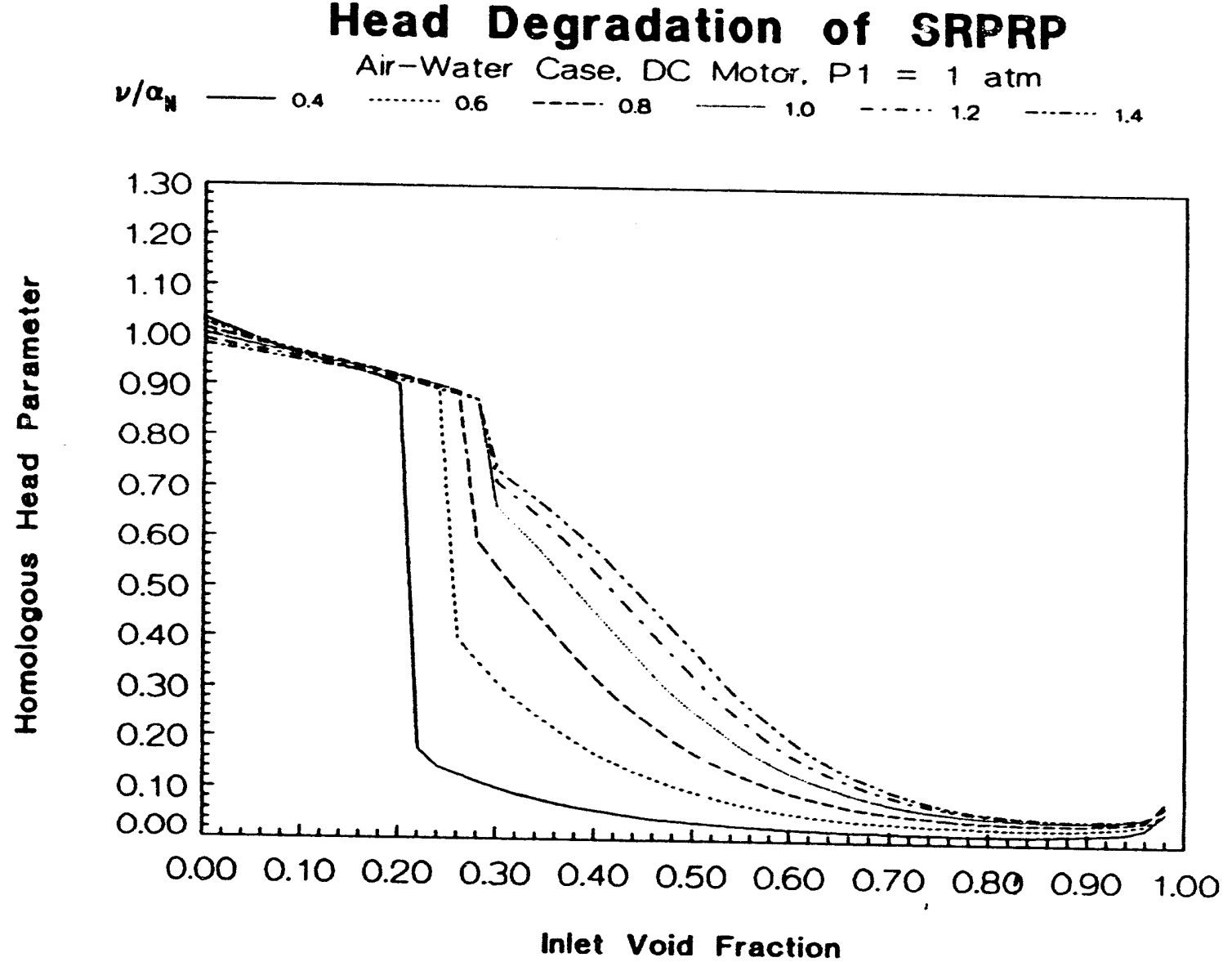


Figure 4.3.2-1d $\quad\left(p_{1}=0.05 \mathrm{~atm}\right)$

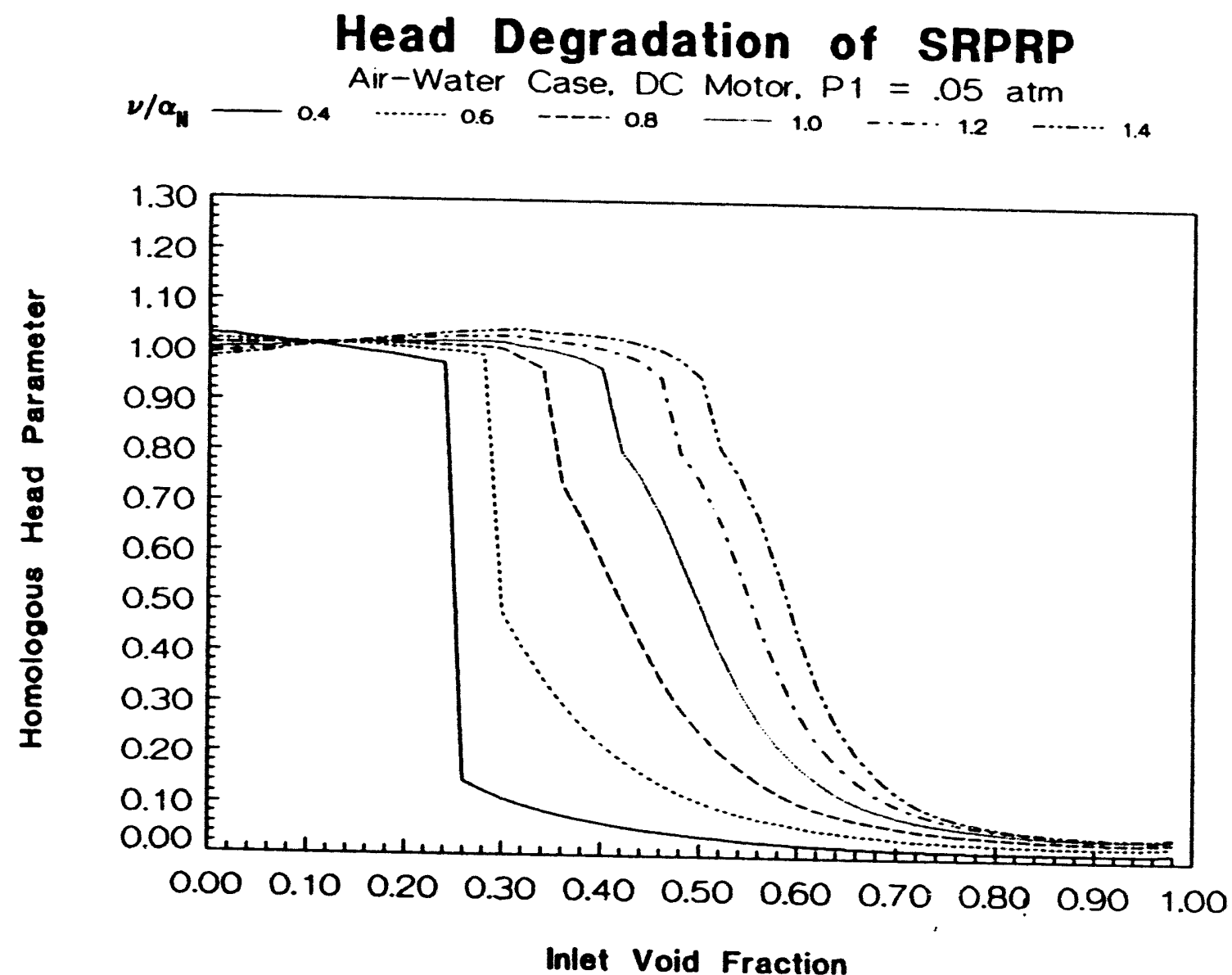


Table 4.3.2-1a $\quad\left(p_{1}=3\right.$ atm $)$

Homologous Head Parameter vs. Inlet Void Fraction for Various Homologous Flow Parameters at Pl $=3 \mathrm{~atm}$ DC Motor Case

\begin{tabular}{|c|c|c|c|c|c|c|}
\hline \multirow{2}{*}{$\begin{array}{l}\text { Inlet } \\
\text { void }\end{array}$} & \multicolumn{6}{|c|}{ Homologous Flow Parameter } \\
\hline & 0.4 & 0.6 & 0.8 & 1.0 & 1.2 & 1.4 \\
\hline $\begin{array}{l}0.00 \\
0.02 \\
0.04 \\
0.06 \\
0.08 \\
0.10 \\
0.12 \\
0.14 \\
0.16 \\
0.18 \\
0.20 \\
0.22 \\
0.24 \\
0.26 \\
0.28 \\
0.30 \\
0.32 \\
0.34 \\
0.36 \\
0.38 \\
0.40 \\
0.42 \\
0.44 \\
0.46 \\
0.48 \\
0.50 \\
0.52 \\
0.54 \\
0.56 \\
0.58 \\
0.60 \\
0.62 \\
0.64 \\
0.66 \\
0.68 \\
0.70 \\
0.72 \\
0.74 \\
0.76 \\
0.78 \\
0.80 \\
0.82 \\
0.84 \\
0.86 \\
0.88 \\
0.90 \\
0.92 \\
0.94 \\
0.96 \\
0.98\end{array}$ & $\begin{array}{l}1.032 \\
1.012 \\
0.996 \\
0.980 \\
0.965 \\
0.951 \\
0.938 \\
0.925 \\
0.913 \\
0.618 \\
0.267 \\
0.172 \\
0.144 \\
0.128 \\
0.125 \\
0.103 \\
0.092 \\
0.083 \\
0.075 \\
0.068 \\
0.062 \\
0.056 \\
0.051 \\
0.047 \\
0.043 \\
0.039 \\
0.036 \\
0.034 \\
0.031 \\
0.029 \\
0.028 \\
0.026 \\
0.025 \\
0.021 \\
0.023 \\
0.022 \\
0.022 \\
0.021 \\
0.021 \\
0.022 \\
0.021 \\
0.022 \\
0.024 \\
0.025 \\
0.027 \\
0.031 \\
0.038 \\
0.051 \\
0.066 \\
0.161\end{array}$ & $\begin{array}{l}1.022 \\
1.008 \\
0.994 \\
0.982 \\
0.969 \\
0.957 \\
0.945 \\
0.934 \\
0.922 \\
0.911 \\
0.901 \\
0.881 \\
0.456 \\
0.384 \\
0.342 \\
0.306 \\
0.273 \\
0.243 \\
0.216 \\
0.193 \\
0.172 \\
0.153 \\
0.137 \\
0.122 \\
0.110 \\
0.098 \\
0.089 \\
0.080 \\
0.072 \\
0.066 \\
0.060 \\
0.056 \\
0.051 \\
0.048 \\
0.045 \\
0.042 \\
0.040 \\
0.038 \\
0.038 \\
0.037 \\
0.036 \\
0.036 \\
0.036 \\
0.038 \\
0.038 \\
0.041 \\
0.047 \\
0.057 \\
0.060 \\
0.134\end{array}$ & $\begin{array}{l}1.012 \\
1.000 \\
0.988 \\
0.976 \\
0.965 \\
0.953 \\
0.942 \\
0.931 \\
0.921 \\
0.910 \\
0.900 \\
0.889 \\
0.872 \\
0.620 \\
0.555 \\
0.511 \\
0.468 \\
0.426 \\
0.386 \\
0.348 \\
0.312 \\
0.279 \\
0.248 \\
0.221 \\
0.196 \\
0.174 \\
0.155 \\
0.138 \\
0.123 \\
0.110 \\
0.099 \\
0.089 \\
0.081 \\
0.073 \\
0.067 \\
0.062 \\
0.058 \\
0.055 \\
0.052 \\
0.050 \\
0.048 \\
0.047 \\
0.047 \\
0.047 \\
0.049 \\
0.050 \\
0.056 \\
0.060 \\
0.076 \\
0.145\end{array}$ & 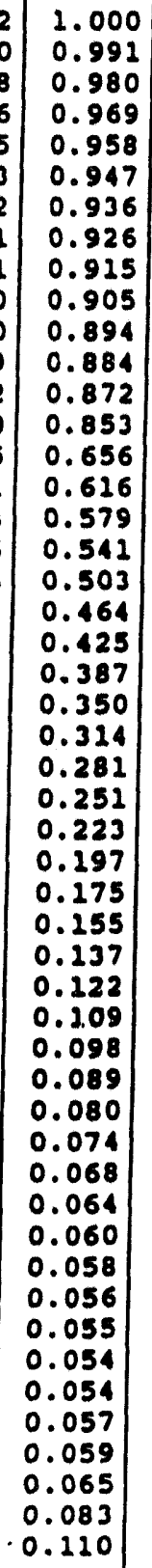 & $\begin{array}{l}0.992 \\
0.982 \\
0.971 \\
0.960 \\
0.950 \\
0.939 \\
0.929 \\
0.928 \\
0.908 \\
0.897 \\
0.887 \\
0.877 \\
0.866 \\
0.850 \\
0.825 \\
0.661 \\
0.629 \\
0.596 \\
0.563 \\
0.528 \\
0.492 \\
0.455 \\
0.118 \\
0.382 \\
0.346 \\
0.311 \\
0.278 \\
0.248 \\
0.219 \\
0.194 \\
0.171 \\
0.251 \\
0.134 \\
0.119 \\
0.106 \\
0.095 \\
0.086 \\
0.078 \\
0.072 \\
0.067 \\
0.064 \\
0.061 \\
0.059 \\
0.058 \\
0.058 \\
0.059 \\
0.062 \\
0.066 \\
0.080 \\
0.134\end{array}$ & $\begin{array}{l}0.983 \\
0.972 \\
0.962 \\
0.951 \\
0.941 \\
0.931 \\
0.920 \\
0.920 \\
0.899 \\
0.889 \\
0.879 \\
0.868 \\
0.858 \\
0.844 \\
0.824 \\
0.680 \\
0.650 \\
0.621 \\
0.591 \\
0.559 \\
0.526 \\
0.492 \\
0.457 \\
0.422 \\
0.386 \\
0.351 \\
0.317 \\
0.283 \\
0.252 \\
0.223 \\
0.197 \\
0.173 \\
0.153 \\
0.134 \\
0.218 \\
0.105 \\
0.094 \\
0.081 \\
0.076 \\
0.070 \\
0.065 \\
0.062 \\
0.059 \\
0.058 \\
0.057 \\
0.057 \\
0.062 \\
0.065 \\
0.077 \\
0.133\end{array}$ \\
\hline
\end{tabular}


Table 4.3.2-1b $\quad\left(p_{1}=2 \mathrm{~atm}\right)$

Homologous Head Parameter vs. Inlet Void Fraction for Varlous Homologous Flow Parameters at Pl = 2 atm DC Motor Case

\begin{tabular}{|c|c|c|c|c|c|c|}
\hline \multirow{2}{*}{$\begin{array}{r}\text { Inlet } \\
\text { vold }\end{array}$} & \multicolumn{6}{|c|}{ Homologous Flow Parameter } \\
\hline & 0.4 & 0.6 & 0.8 & 2.0 & 1.2 & 1.4 \\
\hline $\begin{array}{l}0.38 \\
0.40 \\
0.42 \\
0.44 \\
0.46 \\
0.48 \\
0.50 \\
0.52 \\
0.54 \\
0.56 \\
0.58 \\
0.60 \\
0.62 \\
0.64 \\
0.66 \\
0.68 \\
0.70 \\
0.72 \\
0.74 \\
0.76 \\
0.78 \\
0.80 \\
0.82 \\
0.84 \\
0.86 \\
0.88 \\
0.90 \\
0.92 \\
0.94 \\
0.96 \\
0.98\end{array}$ & $\begin{array}{l}1.032 \\
1.023 \\
0.997 \\
0.982 \\
0.968 \\
0.954 \\
0.941 \\
0.929 \\
0.917 \\
0.904 \\
0.291 \\
0.173 \\
0.144 \\
0.128 \\
0.214 \\
0.102 \\
0.092 \\
0.083 \\
0.075 \\
0.068 \\
0.061 \\
0.056 \\
0.051 \\
0.046 \\
0.042 \\
0.039 \\
0.036 \\
0.033 \\
0.030 \\
0.028 \\
0.027 \\
0.025 \\
0.024 \\
0.023 \\
0.021 \\
0.021 \\
0.020 \\
0.020 \\
0.020 \\
0.020 \\
0.020 \\
0.020 \\
0.021 \\
0.021 \\
0.023 \\
0.026 \\
0.032 \\
0.038 \\
0.056 \\
0.126\end{array}$ & $\begin{array}{l}1.022 \\
1.009 \\
0.996 \\
0.984 \\
0.972 \\
0.960 \\
0.949 \\
0.938 \\
0.927 \\
0.916 \\
0.906 \\
0.893 \\
0.480 \\
0.387 \\
0.345 \\
0.307 \\
0.274 \\
0.244 \\
0.217 \\
0.193 \\
0.272 \\
0.153 \\
0.236 \\
0.122 \\
0.209 \\
0.098 \\
0.088 \\
0.079 \\
0.072 \\
0.065 \\
0.059 \\
0.054 \\
0.050 \\
0.047 \\
0.044 \\
0.041 \\
0.039 \\
0.037 \\
0.036 \\
0.035 \\
0.034 \\
0.034 \\
0.034 \\
0.034 \\
0.036 \\
0.036 \\
0.042 \\
0.047 \\
0.052 \\
0.093\end{array}$ & $\begin{array}{l}1.012 \\
1.001 \\
0.990 \\
0.979 \\
0.968 \\
0.957 \\
0.947 \\
0.937 \\
0.926 \\
0.916 \\
0.906 \\
0.896 \\
0.883 \\
0.856 \\
0.565 \\
0.519 \\
0.475 \\
0.432 \\
0.391 \\
0.351 \\
0.315 \\
0.281 \\
0.250 \\
0.222 \\
0.297 \\
0.174 \\
0.155 \\
0.138 \\
0.122 \\
0.109 \\
0.098 \\
0.088 \\
0.080 \\
0.072 \\
0.066 \\
0.061 \\
0.057 \\
0.053 \\
0.050 \\
0.048 \\
0.046 \\
0.045 \\
0.045 \\
0.045 \\
0.045 \\
0.046 \\
0.050 \\
0.055 \\
0.067 \\
0.135\end{array}$ & $\begin{array}{l}1.000 \\
1 \\
0.992 \\
0.982 \\
0.972 \\
0.962 \\
0.951 \\
0.941 \\
0.932 \\
0.922 \\
0.912 \\
0.902 \\
0.892 \\
0.881 \\
0.866 \\
0.670 \\
0.628 \\
0.591 \\
0.552 \\
0.512 \\
0.472 \\
0.432 \\
0.393 \\
0.354 \\
0.318 \\
0.284 \\
0.252 \\
0.224 \\
0.198 \\
0.175 \\
0.155 \\
0.137 \\
0.1222 \\
0.108 \\
0.097 \\
0.087 \\
0.079 \\
0.073 \\
0.067 \\
0.062 \\
0.059 \\
0.056 \\
0.054 \\
0.052 \\
0.052 \\
0.052 \\
0.053 \\
0.055 \\
0.058 \\
0.069 \\
0.107\end{array}$ & $\begin{array}{l}0.992 \\
0.983 \\
0.973 \\
0.964 \\
0.954 \\
0.944 \\
0.935 \\
0.925 \\
0.915 \\
0.905 \\
0.896 \\
0.886 \\
0.876 \\
0.863 \\
0.844 \\
0.675 \\
0.643 \\
0.610 \\
0.575 \\
0.539 \\
0.502 \\
0.465 \\
0.426 \\
0.388 \\
0.351 \\
0.315 \\
0.281 \\
0.250 \\
0.221 \\
0.195 \\
0.272 \\
0.151 \\
0.134 \\
0.128 \\
0.105 \\
0.094 \\
0.085 \\
0.077 \\
0.071 \\
0.066 \\
0.062 \\
0.059 \\
0.056 \\
0.055 \\
0.055 \\
0.055 \\
0.057 \\
0.062 \\
0.072 \\
0.122\end{array}$ & $\begin{array}{l}0.983 \\
0.974 \\
0.964 \\
0.955 \\
0.946 \\
0.936 \\
0.927 \\
0.91 \\
0.908 \\
0.898 \\
0.888 \\
0.878 \\
0.869 \\
0.857 \\
0.841 \\
0.696 \\
0.666 \\
0.636 \\
0.605 \\
0.573 \\
0.540 \\
0.505 \\
0.468 \\
0.432 \\
0.394 \\
0.358 \\
0.322 \\
0.287 \\
0.255 \\
0.225 \\
0.298 \\
0.174 \\
0.252 \\
0.234 \\
0.118 \\
0.104 \\
0.093 \\
0.083 \\
0.075 \\
0.069 \\
0.064 \\
0.060 \\
0.057 \\
0.056 \\
0.055 \\
0.055 \\
0.056 \\
0.059 \\
0.068 \\
0.103\end{array}$ \\
\hline
\end{tabular}


Table 4.3.2-1c $\quad\left(p_{1}=1 \mathrm{~atm}\right)$

Homologous Head Parameter vs. Inlet Vold Fraction for Varlous Homologous Flow Parameters at PI = 1 atm DC Motor Case

\begin{tabular}{|c|c|c|c|c|c|c|}
\hline \multirow{2}{*}{$\begin{array}{r}\text { Inlet } \\
\text { vold }\end{array}$} & \multicolumn{6}{|c|}{ Homologous Flow Parameter } \\
\hline & 0.4 & 0.6 & 0.8 & 1.0 & 2.2 & 2.4 \\
\hline $\begin{array}{l}0.00 \\
0.02 \\
0.04 \\
0.06 \\
0.08 \\
0.10 \\
0.12 \\
0.14 \\
0.16 \\
0.18 \\
0.20 \\
0.22 \\
0.24 \\
0.26 \\
0.28 \\
0.30 \\
0.32 \\
0.34 \\
0.36 \\
0.38 \\
0.40 \\
0.42 \\
0.44 \\
0.46 \\
0.48 \\
0.50 \\
0.52 \\
0.54 \\
0.56 \\
0.58 \\
0.60 \\
0.62 \\
0.64 \\
0.66 \\
0.68 \\
0.70 \\
0.72 \\
0.74 \\
0.76 \\
0.78 \\
0.80 \\
0.82 \\
0.84 \\
0.86 \\
0.88 \\
0.90 \\
0.92 \\
0.94 \\
0.96 \\
0.98\end{array}$ & $\begin{array}{l}1.032 \\
1.016 \\
2.002 \\
0.988 \\
0.075 \\
0.962 \\
0.950 \\
0.938 \\
0.927 \\
0.926 \\
0.902 \\
0.179 \\
0.144 \\
0.128 \\
0.124 \\
0.202 \\
0.091 \\
0.082 \\
0.074 \\
0.067 \\
0.061 \\
0.055 \\
0.050 \\
0.045 \\
0.041 \\
0.038 \\
0.035 \\
0.032 \\
0.029 \\
0.027 \\
0.025 \\
0.024 \\
0.022 \\
0.021 \\
0.020 \\
0.019 \\
0.018 \\
0.018 \\
0.017 \\
0.027 \\
0.017 \\
0.017 \\
0.027 \\
0.018 \\
0.019 \\
0.020 \\
0.022 \\
0.025 \\
0.034 \\
0.070\end{array}$ & $\begin{array}{l}1.022 \\
1.021 \\
1.000 \\
0.990 \\
0.979 \\
0.969 \\
0.959 \\
0.949 \\
0.939 \\
0.929 \\
0.919 \\
0.909 \\
0.895 \\
0.398 \\
0.353 \\
0.314 \\
0.279 \\
0.247 \\
0.219 \\
0.195 \\
0.173 \\
0.154 \\
0.137 \\
0.122 \\
0.109 \\
0.097 \\
0.087 \\
0.078 \\
0.071 \\
0.064 \\
0.058 \\
0.053 \\
0.049 \\
0.045 \\
0.042 \\
0.040 \\
0.037 \\
0.035 \\
0.034 \\
0.033 \\
0.032 \\
0.032 \\
0.031 \\
0.031 \\
0.031 \\
0.032 \\
0.034 \\
0.037 \\
0.044 \\
0.072 \\
0.037\end{array}$ & $\begin{array}{l}1.022 \\
1.003 \\
0.994 \\
0.985 \\
0.976 \\
0.967 \\
0.958 \\
0.949 \\
0.940 \\
0.930 \\
0.921 \\
0.912 \\
0.902 \\
0.890 \\
0.592 \\
0.544 \\
0.496 \\
0.450 \\
0.405 \\
0.363 \\
0.324 \\
0.288 \\
0.255 \\
0.225 \\
0.299 \\
0.276 \\
0.156 \\
0.138 \\
0.122 \\
0.109 \\
0.097 \\
0.087 \\
0.079 \\
0.071 \\
0.065 \\
0.060 \\
0.055 \\
0.052 \\
0.049 \\
0.046 \\
0.044 \\
0.043 \\
0.042 \\
0.042 \\
0.041 \\
0.042 \\
0.044 \\
0.046 \\
0.051 \\
0.090\end{array}$ & $\begin{array}{l}1.000 \\
0.994 \\
0.986 \\
0.978 \\
0.970 \\
0.962 \\
0.953 \\
0.945 \\
0.937 \\
0.928 \\
0.919 \\
0.911 \\
0.902 \\
0.891 \\
0.877 \\
0.662 \\
0.623 \\
0.583 \\
0.541 \\
0.198 \\
0.454 \\
0.411 \\
0.369 \\
0.329 \\
0.292 \\
0.258 \\
0.228 \\
0.201 \\
0.177 \\
0.156 \\
0.137 \\
0.121 \\
0.108 \\
0.096 \\
0.086 \\
0.078 \\
0.071 \\
0.065 \\
0.061 \\
0.057 \\
0.054 \\
0.051 \\
0.050 \\
0.019 \\
0.018 \\
0.048 \\
0.049 \\
0.052 \\
0.058 \\
0.081 \\
0.04\end{array}$ & 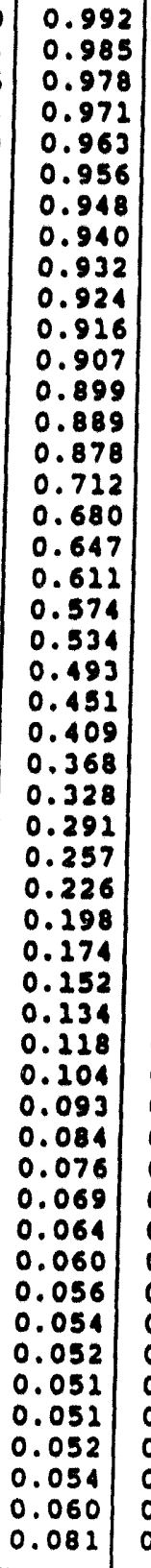 & $\begin{array}{l}0.983 \\
0.976 \\
0.970 \\
0.963 \\
0.956 \\
0.949 \\
0.912 \\
0.934 \\
0.927 \\
0.919 \\
0.912 \\
0.903 \\
0.894 \\
0.885 \\
0.875 \\
0.738 \\
0.707 \\
0.678 \\
0.647 \\
0.614 \\
0.578 \\
0.511 \\
0.502 \\
0.461 \\
0.420 \\
0.3379 \\
0.339 \\
0.300 \\
0.265 \\
0.232 \\
0.203 \\
0.177 \\
0.154 \\
0.134 \\
0.118 \\
0.203 \\
0.092 \\
0.082 \\
0.074 \\
0.067 \\
0.062 \\
0.058 \\
0.055 \\
0.052 \\
0.051 \\
0.051 \\
0.052 \\
0.053 \\
0.056 \\
0.083 \\
\end{array}$ \\
\hline
\end{tabular}


Table 4.3.2-1c

$\left(p_{1}=1 \mathbf{a t m}\right)$

Homologous Head Parameter ve. Inlet Vold Fraction for Varlous Homologous Flow Parameters at P1 - 1 atm DC Motor Case

\begin{tabular}{|c|c|c|c|c|c|c|}
\hline \multirow{2}{*}{$\begin{array}{r}\text { Indet } \\
\text { vold }\end{array}$} & \multicolumn{6}{|c|}{ Homolngous Flow Parameter } \\
\hline & 0.4 & 0.6 & 0.8 & 1.0 & 2.2 & 1.4 \\
\hline $\begin{array}{l}0.00 \\
0.02 \\
0.04 \\
0.06 \\
0.08 \\
0.10 \\
0.22 \\
0.24 \\
0.26 \\
0.28 \\
0.20 \\
0.22 \\
0.24 \\
0.26 \\
0.28 \\
0.30 \\
0.32 \\
0.34 \\
0.36 \\
0.38 \\
0.40 \\
0.42 \\
0.44 \\
0.46 \\
0.48 \\
0.50 \\
0.32 \\
0.54 \\
0.56 \\
0.58 \\
0.60 \\
0.62 \\
0.64 \\
0.66 \\
0.68 \\
0.70 \\
0.72 \\
0.74 \\
0.76 \\
0.78 \\
0.80 \\
0.82 \\
0.84 \\
0.86 \\
0.88 \\
0.90 \\
0.92 \\
0.94 \\
0.96 \\
0.98\end{array}$ & $\begin{array}{l}1.032 \\
1.026 \\
1.002 \\
0.988 \\
0.075 \\
0.962 \\
0.950 \\
0.938 \\
0.927 \\
0.916 \\
0.902 \\
0.179 \\
0.141 \\
0.128 \\
0.214 \\
0.102 \\
0.091 \\
0.082 \\
0.074 \\
0.067 \\
0.061 \\
0.055 \\
0.050 \\
0.045 \\
0.041 \\
0.038 \\
0.035 \\
0.032 \\
0.029 \\
0.027 \\
0.025 \\
0.024 \\
0.022 \\
0.021 \\
0.020 \\
0.019 \\
0.018 \\
0.028 \\
0.027 \\
0.017 \\
0.017 \\
0.027 \\
0.017 \\
0.018 \\
0.019 \\
0.020 \\
0.022 \\
0.025 \\
0.034 \\
0.070\end{array}$ & $\begin{array}{l}1.022 \\
1.012 \\
1.000 \\
0.990 \\
0.979 \\
0.869 \\
0.959 \\
0.949 \\
0.939 \\
0.929 \\
0.929 \\
0.909 \\
0.895 \\
0.398 \\
0.353 \\
0.314 \\
0.279 \\
0.247 \\
0.219 \\
0.195 \\
0.173 \\
0.154 \\
0.137 \\
0.122 \\
0.109 \\
0.097 \\
0.087 \\
0.078 \\
0.071 \\
0.064 \\
0.058 \\
0.053 \\
0.049 \\
0.045 \\
0.042 \\
0.040 \\
0.037 \\
0.035 \\
0.031 \\
0.033 \\
0.032 \\
0.031 \\
0.031 \\
0.031 \\
0.031 \\
0.032 \\
0.034 \\
0.037 \\
0.044 \\
0.072\end{array}$ & $\begin{array}{l}1.012 \\
1.003 \\
0.994 \\
0.985 \\
0.976 \\
0.967 \\
0.958 \\
0.949 \\
0.940 \\
0.930 \\
0.922 \\
0.912 \\
0.902 \\
0.890 \\
0.592 \\
0.544 \\
0.496 \\
0.150 \\
0.403 \\
0.363 \\
0.324 \\
0.288 \\
0.255 \\
0.225 \\
0.199 \\
0.176 \\
0.256 \\
0.238 \\
0.222 \\
0.209 \\
0.097 \\
0.087 \\
0.079 \\
0.072 \\
0.065 \\
0.060 \\
0.053 \\
0.052 \\
0.049 \\
0.046 \\
0.044 \\
0.043 \\
0.042 \\
0.041 \\
0.042 \\
0.042 \\
0.044 \\
0.046 \\
0.051 \\
0.090\end{array}$ & $\begin{array}{l}1.000 \\
0.994 \\
0.986 \\
0.978 \\
0.970 \\
0.952 \\
0.953 \\
0.945 \\
0.937 \\
0.928 \\
0.919 \\
0.912 \\
0.902 \\
0.891 \\
0.877 \\
0.662 \\
0.623 \\
0.583 \\
0.512 \\
0.198 \\
0.454 \\
0.412 \\
0.369 \\
0.329 \\
0.292 \\
0.258 \\
0.228 \\
0.201 \\
0.177 \\
0.136 \\
0.237 \\
0.222 \\
0.208 \\
0.096 \\
0.086 \\
0.078 \\
0.071 \\
0.065 \\
0.061 \\
0.057 \\
0.054 \\
0.052 \\
0.050 \\
0.049 \\
0.048 \\
0.048 \\
0.019 \\
0.052 \\
0.058 \\
0.081\end{array}$ &  & $\begin{array}{l}0.983 \\
0.976 \\
0.970 \\
0.963 \\
0.956 \\
0.949 \\
0.942 \\
0.934 \\
0.927 \\
0.919 \\
0.921 \\
0.903 \\
0.894 \\
0.885 \\
0.873 \\
0.738 \\
0.707 \\
0.678 \\
0.647 \\
0.614 \\
0.578 \\
0.542 \\
0.502 \\
0.461 \\
0.420 \\
0.379 \\
0.339 \\
0.300 \\
0.265 \\
0.232 \\
0.203 \\
0.177 \\
0.254 \\
0.234 \\
0.118 \\
0.103 \\
0.092 \\
0.082 \\
0.074 \\
0.067 \\
0.062 \\
0.058 \\
0.055 \\
0.052 \\
0.051 \\
0.051 \\
0.052 \\
0.053 \\
0.056 \\
0.083\end{array}$ \\
\hline
\end{tabular}


Table 4.3.2-2a $\quad\left(p_{1}=3 \mathrm{~atm}\right)$

Discharge Vold Fraction ve. Inlet vold rraction for Varlous Homologous Flow Parameters at P1 - 3 atm DC Motor Case

\begin{tabular}{|c|c|c|c|c|c|c|}
\hline \multirow{2}{*}{$\begin{array}{l}\text { Inlet } \\
\text { Vold }\end{array}$} & \multicolumn{6}{|c|}{ Homologous Flow Paranete } \\
\hline & 0.4 & 0.6 & 0.8 & 2.0 & 1.2 & 2.4 \\
\hline $\begin{array}{l}0.00 \\
0.02 \\
0.04 \\
0.06 \\
0.08 \\
0.10 \\
0.12 \\
0.14 \\
0.16 \\
0.18 \\
0.20 \\
0.22 \\
0.24 \\
0.26 \\
0.28 \\
0.30 \\
0.32 \\
0.34 \\
0.36 \\
0.38 \\
0.40 \\
0.42 \\
0.44 \\
0.46 \\
0.48 \\
0.50 \\
0.52 \\
0.54 \\
0.56 \\
0.58 \\
0.60 \\
0.62 \\
0.64 \\
0.66 \\
0.68 \\
0.70 \\
0.72 \\
0.74 \\
0.76 \\
0.78 \\
0.80 \\
0.82 \\
0.84 \\
0.86 \\
0.88 \\
0.90 \\
0.92 \\
0.94 \\
0.98\end{array}$ & $\begin{array}{l}0.00 \\
0.03 \\
0.07 \\
0.12 \\
0.14 \\
0.16 \\
0.19 \\
0.22 \\
0.24 \\
0.76 \\
0.87 \\
0.88 \\
0.89 \\
0.89 \\
0.90 \\
0.90 \\
0.91 \\
0.91 \\
0.92 \\
0.92 \\
0.93 \\
0.93 \\
0.93 \\
0.94 \\
0.94 \\
0.94 \\
0.94 \\
0.95 \\
0.95 \\
0.95 \\
0.96 \\
0.96 \\
0.96 \\
0.96 \\
0.97 \\
0.97 \\
0.97 \\
0.97 \\
0.98 \\
0.98 \\
0.98 \\
0.98 \\
0.98 \\
0.99 \\
0.99 \\
0.99 \\
0.99 \\
0.00\end{array}$ & $\begin{array}{l}0.00 \\
0.03 \\
0.05 \\
0.08 \\
0.10 \\
0.23 \\
0.15 \\
0.18 \\
0.20 \\
0.22 \\
0.24 \\
0.28 \\
0.73 \\
0.76 \\
0.78 \\
0.80 \\
0.82 \\
0.83 \\
0.84 \\
0.85 \\
0.87 \\
0.87 \\
0.88 \\
0.89 \\
0.90 \\
0.90 \\
0.92 \\
0.92 \\
0.92 \\
0.93 \\
0.93 \\
0.93 \\
0.94 \\
0.94 \\
0.95 \\
0.95 \\
0.95 \\
0.96 \\
0.96 \\
0.96 \\
0.97 \\
0.97 \\
0.98 \\
0.98 \\
0.98 \\
0.99 \\
0.99 \\
0.99 \\
0.00\end{array}$ & $\begin{array}{l}0.00 \\
0.02 \\
0.05 \\
0.07 \\
0.09 \\
0.12 \\
0.14 \\
0.16 \\
0.18 \\
0.20 \\
0.22 \\
0.25 \\
0.27 \\
0.57 \\
0.61 \\
0.64 \\
0.68 \\
0.72 \\
0.74 \\
0.76 \\
0.78 \\
0.80 \\
0.81 \\
0.83 \\
0.84 \\
0.86 \\
0.87 \\
0.88 \\
0.88 \\
0.89 \\
0.90 \\
0.91 \\
0.92 \\
0.92 \\
0.93 \\
0.93 \\
0.94 \\
0.94 \\
0.95 \\
0.95 \\
0.96 \\
0.96 \\
0.97 \\
0.97 \\
0.98 \\
0.98 \\
0.98 \\
0.99 \\
0.99 \\
1.00\end{array}$ & 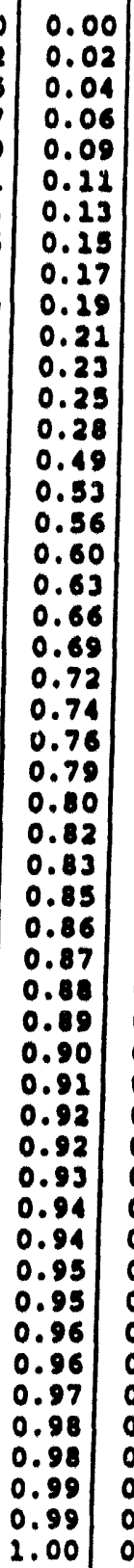 & $\begin{array}{l}0.00 \\
0.02 \\
0.04 \\
0.06 \\
0.08 \\
0.10 \\
0.12 \\
0.14 \\
0.16 \\
0.28 \\
0.20 \\
0.22 \\
0.25 \\
0.27 \\
0.30 \\
0.46 \\
0.49 \\
0.52 \\
0.56 \\
0.59 \\
0.62 \\
0.65 \\
0.68 \\
0.70 \\
0.73 \\
0.75 \\
0.77 \\
0.79 \\
0.81 \\
0.83 \\
0.84 \\
0.85 \\
0.87 \\
0.88 \\
0.89 \\
0.90 \\
0.91 \\
0.92 \\
0.92 \\
0.93 \\
0.94 \\
0.94 \\
0.95 \\
0.96 \\
0.96 \\
0.97 \\
0.989\end{array}$ & $\begin{array}{l}0.00 \\
0.02 \\
0.04 \\
0.06 \\
0.08 \\
0.20 \\
0.12 \\
0.14 \\
0.26 \\
0.28 \\
0.20 \\
0.22 \\
0.24 \\
0.26 \\
0.29 \\
0.42 \\
0.45 \\
0.48 \\
0.51 \\
0.54 \\
0.57 \\
0.60 \\
0.63 \\
0.66 \\
0.69 \\
0.71 \\
0.73 \\
0.76 \\
0.78 \\
0.80 \\
0.82 \\
0.83 \\
0.84 \\
0.86 \\
0.87 \\
0.88 \\
0.89 \\
0.90 \\
0.91 \\
0.92 \\
0.93 \\
0.94 \\
0.94 \\
0.95 \\
0.96 \\
0.97 \\
0.97 \\
0.98 \\
0.99 \\
0.99\end{array}$ \\
\hline
\end{tabular}


Table 4.3.2-2b $\quad\left(p_{1}=2 \mathrm{~atm}\right)$

Dlscharge vold Fraction va. Inlet Vold Fraction for Varlous Homologous Flow Parameters at $P_{1}=2 \mathrm{~atm}$ DC Motor Case

\begin{tabular}{|c|c|c|c|c|c|c|}
\hline \multirow{2}{*}{$\begin{array}{l}\text { Inlet } \\
\text { vold }\end{array}$} & \multicolumn{6}{|c|}{ Homologous Flow Paramete } \\
\hline & 0.4 & 0.6 & 0.8 & 1.0 & 2.2 & 2.4 \\
\hline $\begin{array}{l}0.00 \\
0.02 \\
0.04 \\
0.06 \\
0.08 \\
0.20 \\
0.22 \\
0.14 \\
0.16 \\
0.18 \\
0.20 \\
0.22 \\
0.24 \\
0.26 \\
0.28 \\
0.30 \\
0.332 \\
0.34 \\
0.36 \\
0.38 \\
0.40 \\
0.42 \\
0.44 \\
0.46\end{array}$ & $\begin{array}{l}0.00 \\
0.03 \\
0.07 \\
0.10 \\
0.23 \\
0.16 \\
0.18 \\
0.21 \\
0.23 \\
0.26 \\
0.86 \\
0.88 \\
0.89 \\
0.89 \\
0.90 \\
0.90 \\
0.91 \\
0.91 \\
0.92 \\
0.92 \\
0.93 \\
0.93 \\
0.93 \\
0.94 \\
0.94 \\
0.94 \\
0.94 \\
0.95 \\
0.95 \\
0.95 \\
0.96 \\
0.96 \\
0.96 \\
0.96 \\
0.97 \\
0.97 \\
0.97 \\
0.97 \\
0.98 \\
0.98 \\
0.98 \\
0.98 \\
0.98 \\
0.99 \\
0.99 \\
0.99 \\
0.99 \\
0.99 \\
1.00 \\
1.00\end{array}$ & $\begin{array}{l}0.00 \\
0.03 \\
0.03 \\
0.07 \\
0.10 \\
0.22 \\
0.21 \\
0.27 \\
0.19 \\
0.21 \\
0.23 \\
0.26 \\
0.73 \\
0.76 \\
0.78 \\
0.80 \\
0.81 \\
0.83 \\
0.84 \\
0.85 \\
0.86 \\
0.87 \\
0.88 \\
0.89 \\
0.90 \\
0.90 \\
0.91 \\
0.92 \\
0.92 \\
0.99 \\
0.93 \\
0.93 \\
0.94 \\
0.94 \\
0.95 \\
0.95 \\
0.95 \\
0.96 \\
0.96 \\
0.96 \\
0.97 \\
0.97 \\
0.98 \\
0.98 \\
0.98 \\
0.99 \\
0.99 \\
0.99 \\
0.99 \\
1.00\end{array}$ & $\begin{array}{l}0.00 \\
0.02 \\
0.04 \\
0.06 \\
0.09 \\
0.12 \\
0.13 \\
0.15 \\
0.17 \\
0.219 \\
0.22 \\
0.23 \\
0.26 \\
0.29 \\
0.60 \\
0.61 \\
0.67 \\
0.70 \\
0.73 \\
0.76 \\
0.78 \\
0.80 \\
0.81 \\
0.83 \\
0.84 \\
0.86 \\
0.87 \\
0.88 \\
0.88 \\
0.89 \\
0.90 \\
0.92 \\
0.92 \\
0.92 \\
0.93 \\
0.93 \\
0.94 \\
0.94 \\
0.95 \\
0.95 \\
0.96 \\
0.96 \\
0.97 \\
0.97 \\
0.98 \\
0.98 \\
0.98 \\
0.99 \\
0.99 \\
1.00\end{array}$ & $\begin{array}{l}0.00 \\
0.02 \\
0.04 \\
0.06 \\
0.08 \\
0.20 \\
0.12 \\
0.14 \\
0.16 \\
0.18 \\
0.20 \\
0.22 \\
0.21 \\
0.27 \\
0.18 \\
0.51 \\
0.55 \\
0.39 \\
0.62 \\
0.65 \\
0.69 \\
0.72 \\
0.74 \\
0.76 \\
0.78 \\
0.80 \\
0.82 \\
0.83 \\
0.83 \\
0.86 \\
0.87 \\
0.88 \\
0.89 \\
0.90 \\
0.92 \\
0.92 \\
0.92 \\
0.93 \\
0.94 \\
0.94 \\
0.95 \\
0.95 \\
0.96 \\
0.96 \\
0.97 \\
0.98 \\
0.98 \\
0.99 \\
0.99 \\
1.00\end{array}$ & $\begin{array}{l}0.00 \\
0.02 \\
0.04 \\
0.06 \\
0.08 \\
0.09 \\
0.22 \\
0.23 \\
0.25 \\
0.27 \\
0.19 \\
0.21 \\
0.23 \\
0.28 \\
0.28 \\
0.14 \\
0.48 \\
0.51 \\
0.55 \\
0.58 \\
0.62 \\
0.64 \\
0.67 \\
0.70 \\
0.73 \\
0.75 \\
0.77 \\
0.79 \\
0.81 \\
0.83 \\
0.84 \\
0.85 \\
0.87 \\
0.88 \\
0.89 \\
0.90 \\
0.92 \\
0.92 \\
0.92 \\
0.93 \\
0.94 \\
0.94 \\
0.95 \\
0.96 \\
0.96 \\
0.97 \\
0.98 \\
0.98 \\
0.99 \\
0.99\end{array}$ & 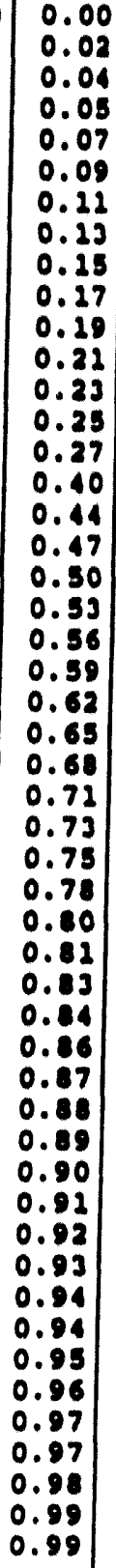 \\
\hline
\end{tabular}


Table 4.3.2-2c $\quad\left(p_{1}=1 \mathrm{~atm}\right)$

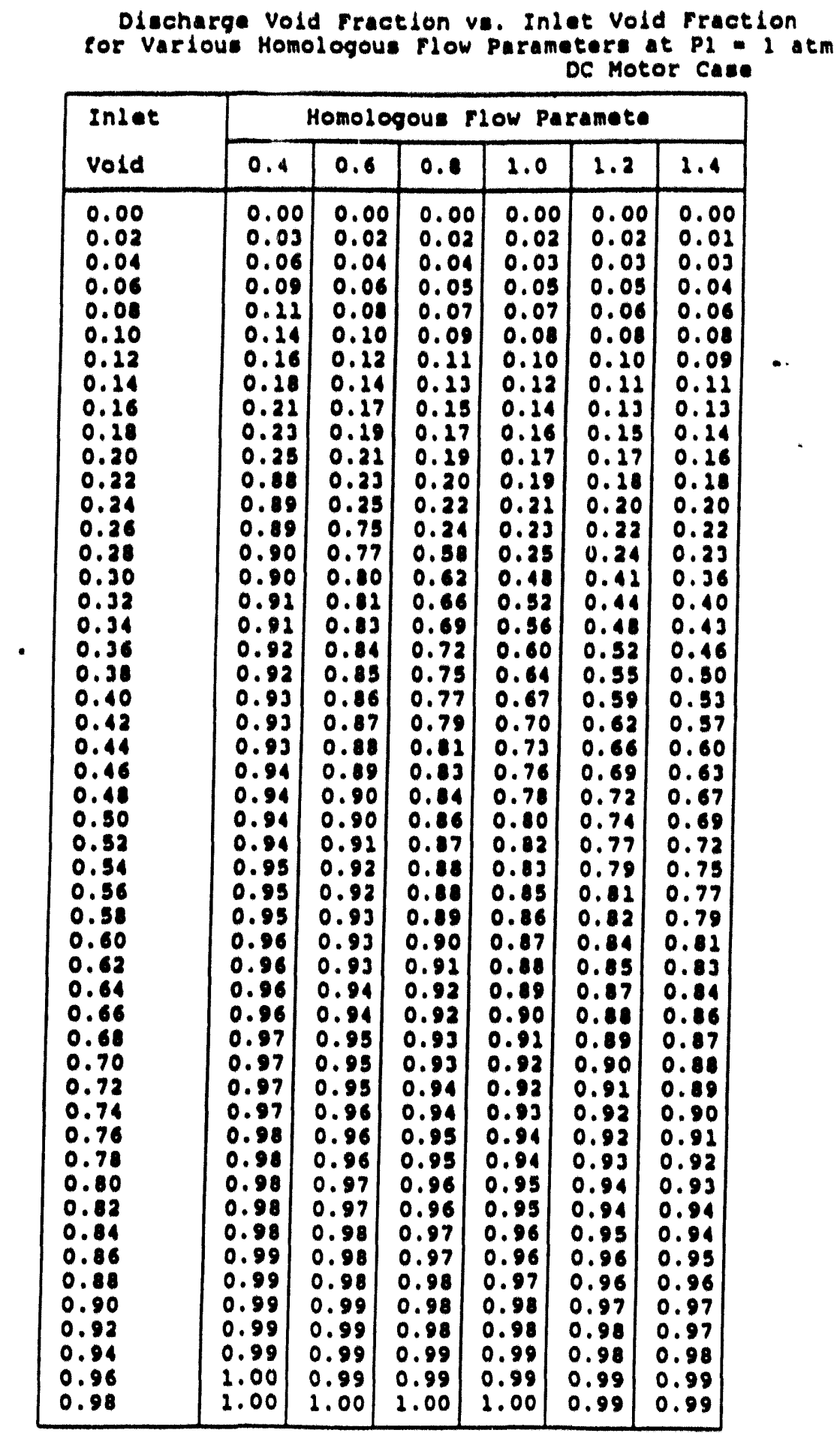


Table 4.3.2-2d $\quad\left(p_{1}=0.05 \mathrm{~atm}\right)$

Dlecharge Vold rraction ve. Inlet vold Fraction tor Varlous Homologous Flow Paraneters at Pl 0.05 atm DC Motor Case

\begin{tabular}{|c|c|c|c|c|c|c|}
\hline \multirow{2}{*}{$\begin{array}{l}\text { Indet } \\
\text { Vold }\end{array}$} & \multicolumn{6}{|c|}{ Homologous Flow Paramete } \\
\hline & 0.4 & 0.6 & 0.1 & 2.0 & 2.2 & 1.1 \\
\hline  & 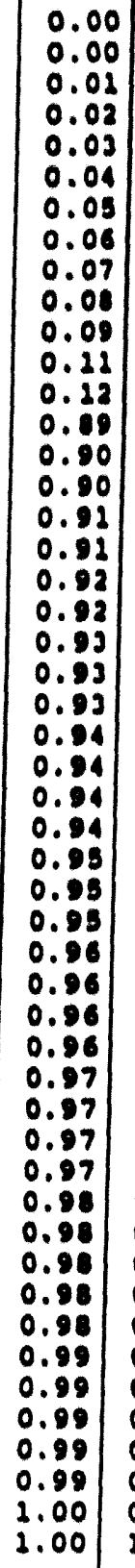 & 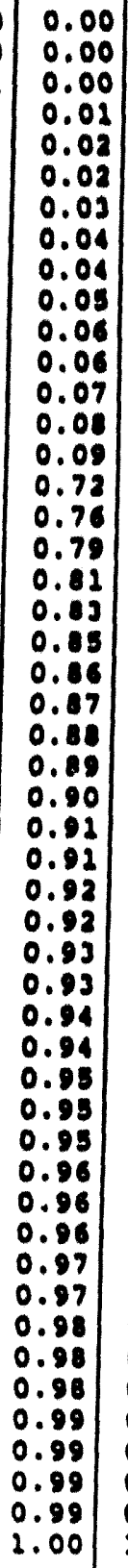 &  & 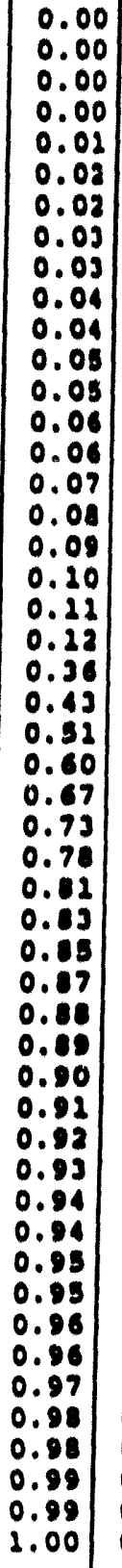 & 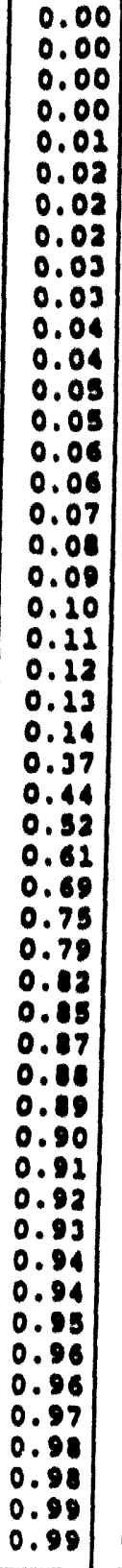 & 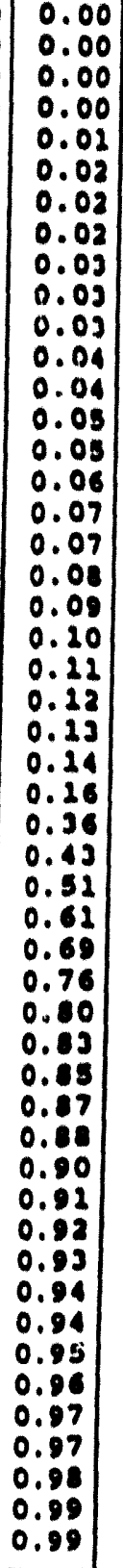 \\
\hline
\end{tabular}


Table 4.3.2-3n $\quad\left(p_{1}=3 \mathrm{~atm}\right)$

static presuure Rlse in pole ve. Inlet vold praction

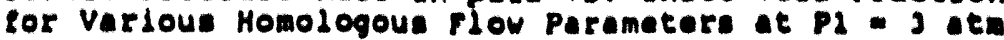
DC Motor Case

\begin{tabular}{|c|c|c|c|c|c|c|}
\hline \multirow{2}{*}{$\begin{array}{l}\text { Indet } \\
\text { vold } \\
\text { ereotion }\end{array}$} & \multicolumn{6}{|c|}{ Homologous Flow Paraneter } \\
\hline & 0.4 & 0.6 & 0.8 & 1.0 & 2.2 & 2.4 \\
\hline 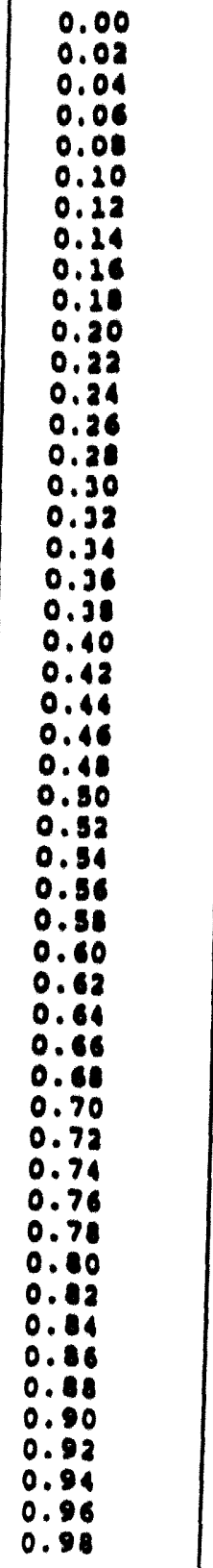 & 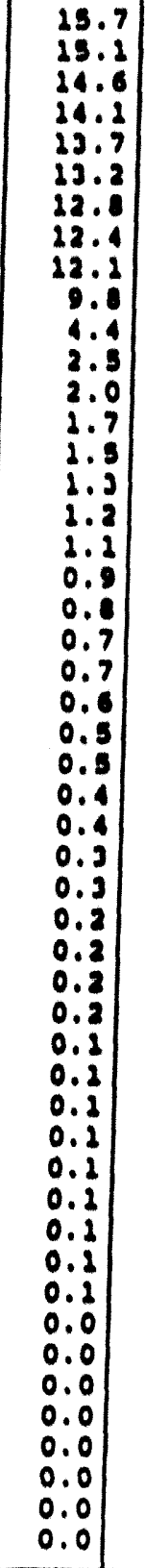 & 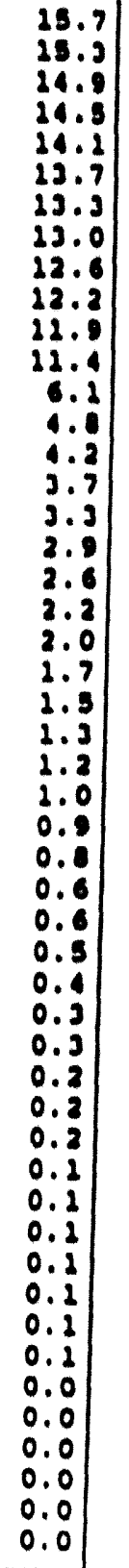 & 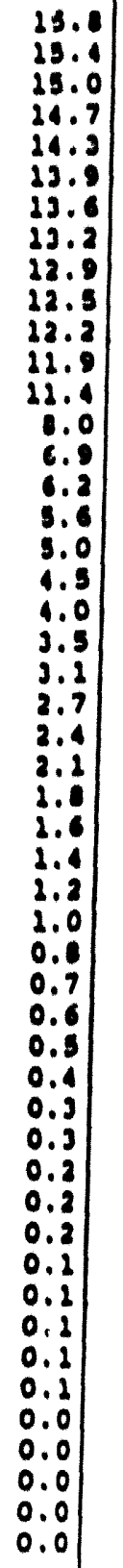 &  & 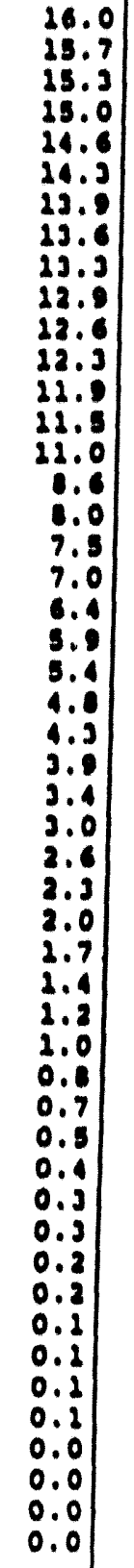 & 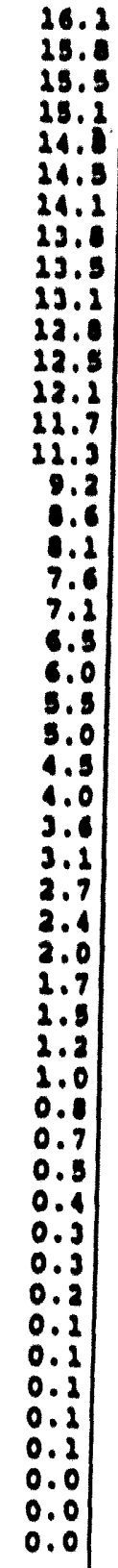 \\
\hline
\end{tabular}


Table 4.3.2-3b $\quad\left(p_{1}=2 \mathrm{~atm}\right)$

statle Presenure Rise In pele ve. Inlet Vold rraction for verlous Homologous Fiow parameters at P1 - 2 at

DC Motor Case

\begin{tabular}{|c|c|c|c|c|c|c|}
\hline \multirow{2}{*}{$\begin{array}{l}\text { Inlet } \\
\text { vold } \\
\text { eraetion }\end{array}$} & \multicolumn{6}{|c|}{ Howologous Flow Parameter } \\
\hline & 0.4 & 0.6 & 0.8 & 2.0 & 2.2 & 2.4 \\
\hline 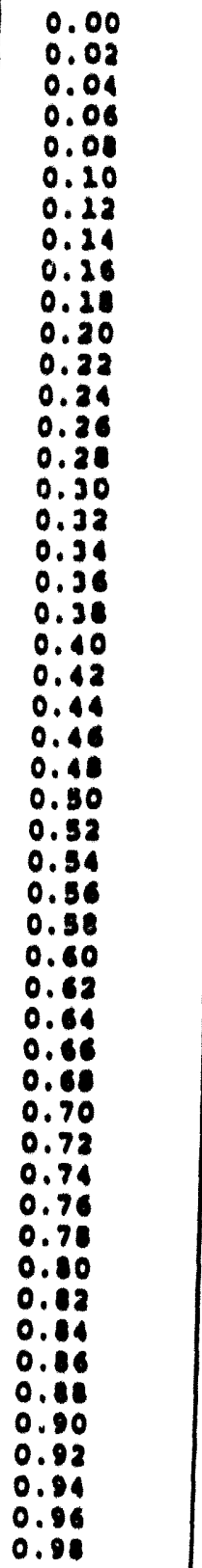 &  & 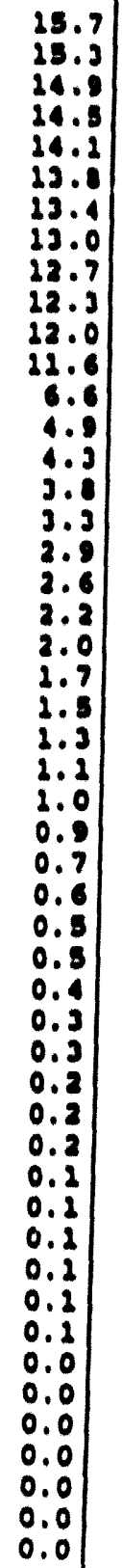 & 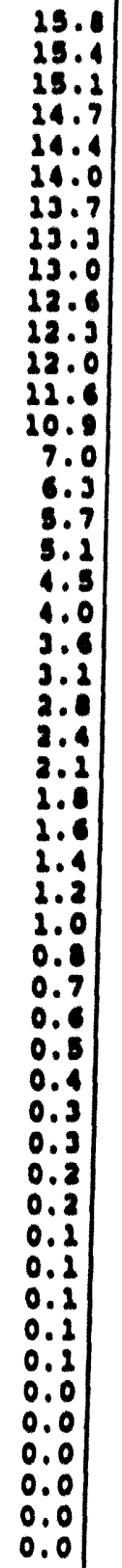 & 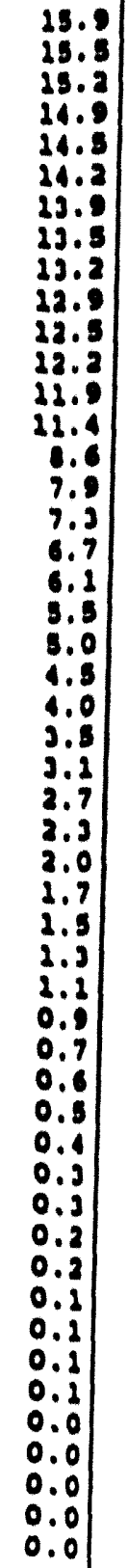 & 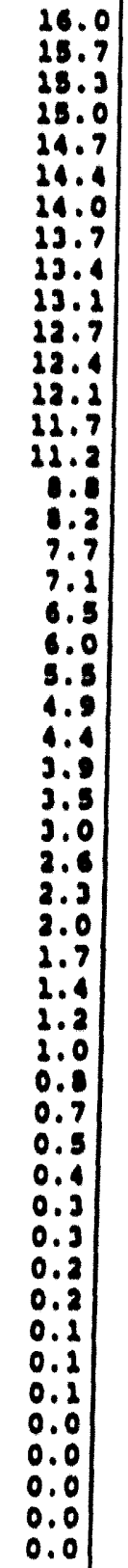 & 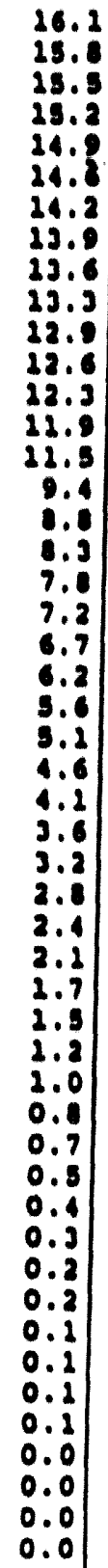 \\
\hline
\end{tabular}


Table 4.3.2-3c

$\left(p_{1}=1\right.$ atm $)$

static Preseure Rlee in pela ve. Inlet vold Fraction for Varlous Homologous Flow Parametere at $P 1=1 \mathrm{~atm}$ DC Motor Case

\begin{tabular}{|c|c|c|c|c|c|c|}
\hline \multirow{2}{*}{$\begin{array}{l}\text { Inlet } \\
\text { vold } \\
\text { eraction }\end{array}$} & \multicolumn{6}{|c|}{ Homologous Flow Parameter } \\
\hline & 0.4 & 0.6 & 0.8 & 2.0 & 1.2 & 1.4 \\
\hline $\begin{array}{l}0.00 \\
0.02 \\
0.04 \\
0.04 \\
0.08 \\
0.10 \\
0.12 \\
0.14 \\
0.16 \\
0.18 \\
0.20 \\
0.22 \\
0.24 \\
0.26 \\
0.28 \\
0.30 \\
0.32\end{array}$ & 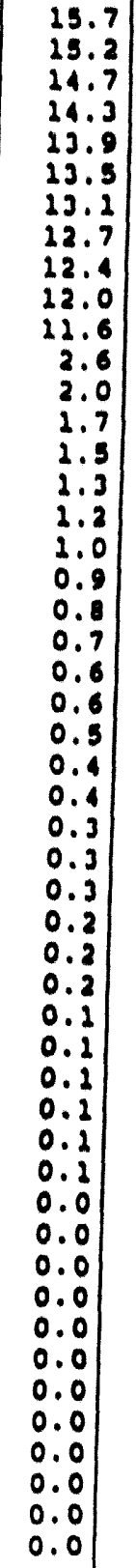 & 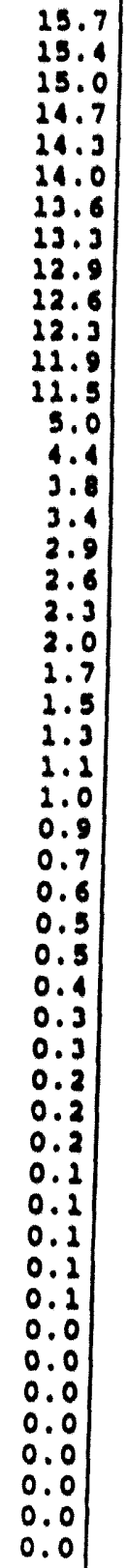 & 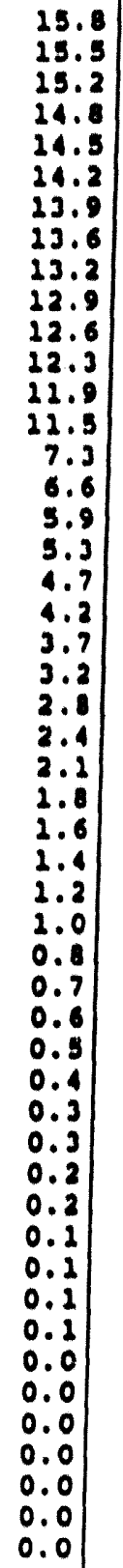 & 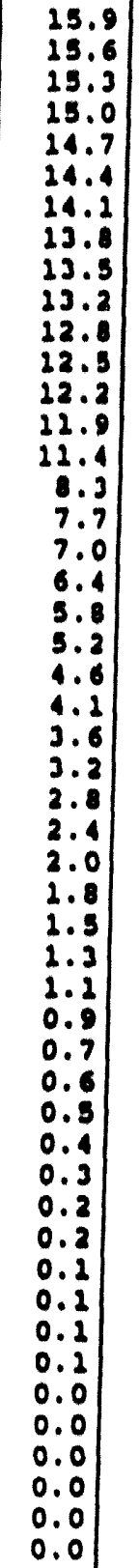 &  &  \\
\hline
\end{tabular}


Table 4.3.2-3d

$$
\left(p_{1}=0.05 \mathrm{~atm}\right)
$$

static Pressure Rise in peia va. Inlet Vold Fraction for Varlous Homologous Flow Parameters at PI $=0.05$ atm DC Motor Case

\begin{tabular}{|c|c|c|c|c|c|c|}
\hline \multirow{2}{*}{$\begin{array}{l}\text { Indet } \\
\text { vold } \\
\text { eraetion }\end{array}$} & \multicolumn{3}{|c|}{ Homologous } & \multicolumn{3}{|c|}{ Flow Parameter } \\
\hline & 0.4 & 0.6 & 0.8 & 1.0 & $1 \cdot 2$ & 1.4 \\
\hline $\begin{array}{l}0.00 \\
0.02 \\
0.04 \\
0.06 \\
0.08 \\
0.10 \\
0.12 \\
0.24 \\
0.26 \\
0.18 \\
0.20 \\
0.22 \\
0.24 \\
0.26 \\
0.28 \\
0.30 \\
0.32 \\
0.34 \\
0.36 \\
0.38 \\
0.40 \\
0.42 \\
0.44 \\
0.16 \\
0.48 \\
0.50 \\
0.32 \\
0.54 \\
0.36 \\
0.38 \\
0.60 \\
0.62 \\
0.64 \\
0.66 \\
0.88 \\
0.70 \\
0.72 \\
0.74 \\
0.76 \\
0.78 \\
0.80 \\
0.82 \\
0.84 \\
0.96 \\
0.88 \\
0.90 \\
0.92 \\
0.94 \\
0.96 \\
0.98\end{array}$ & 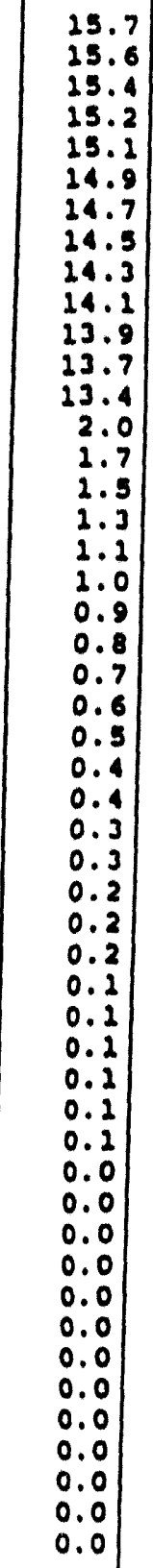 & 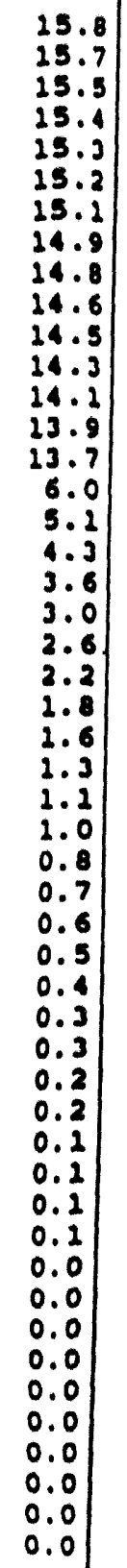 & 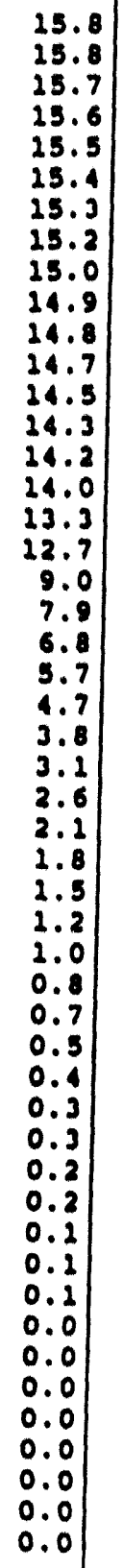 & 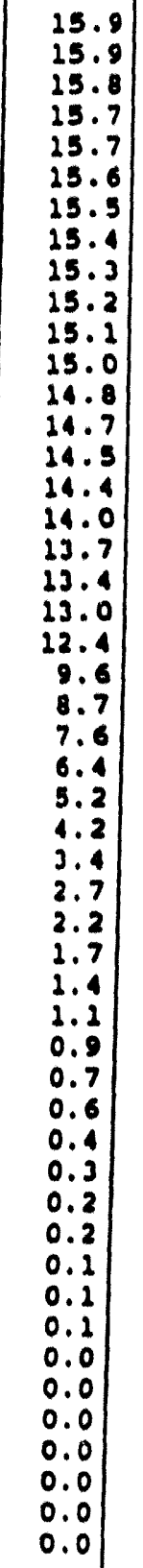 & 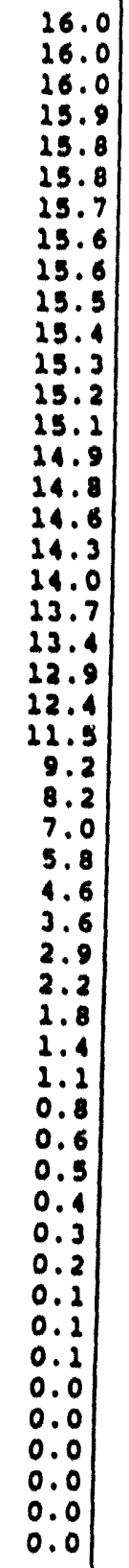 & 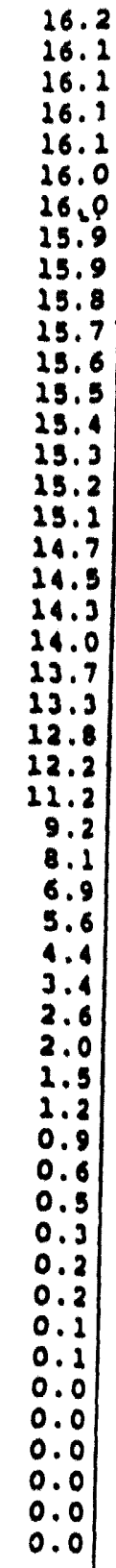 \\
\hline
\end{tabular}


Table 4.3.2-4a $\quad\left(p_{1}=3 \mathrm{~atm}\right)$

Normalized Relative Velocity of Water at Vane Exit vs. Inlet Void Fraction for Various Homologous Flow Parameters at Pl $=3$ atm

DC Motor Case

\begin{tabular}{|c|c|c|c|c|c|c|}
\hline \multirow{2}{*}{$\begin{array}{l}\text { Inlet } \\
\text { Void } \\
\text { Fraction }\end{array}$} & \multicolumn{6}{|c|}{ Homologous Flow Parameter } \\
\hline & 0.4 & 0.6 & 0.8 & 1.0 & 1.2 & 1.4 \\
\hline $\begin{array}{l}0.00 \\
0.02 \\
0.04 \\
0.04 \\
0.08\end{array}$ & 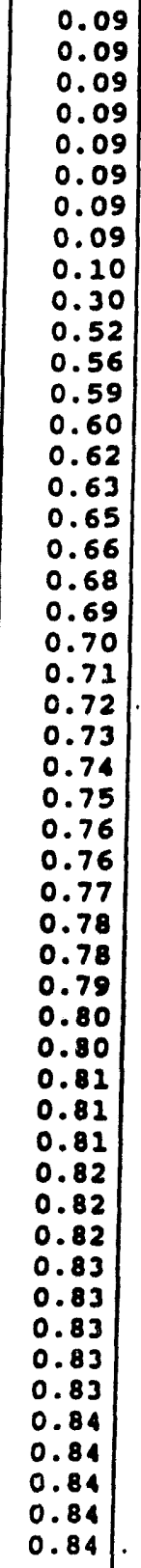 & $\begin{array}{l}0.13 \\
0.13 \\
0.23 \\
0.13 \\
0.13 \\
0.13 \\
0.13 \\
0.14 \\
0.14 \\
0.14 \\
0.14 \\
0.14 \\
0.37 \\
0.40 \\
0.43 \\
0.45 \\
0.45 \\
0.48 \\
0.50 \\
0.53 \\
0.55 \\
0.58 \\
0.00 \\
0.60 \\
0.62 \\
0.04 \\
0.65 \\
0.67 \\
0.69 \\
0.70 \\
0.72\end{array}$ & 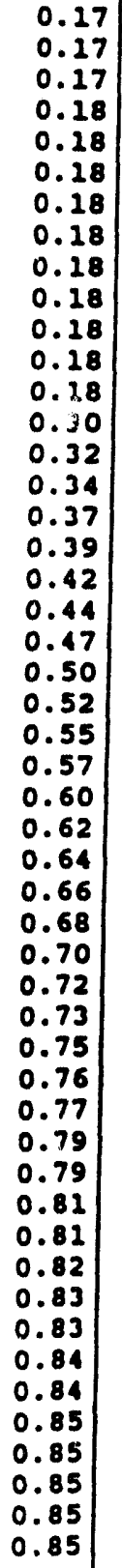 & 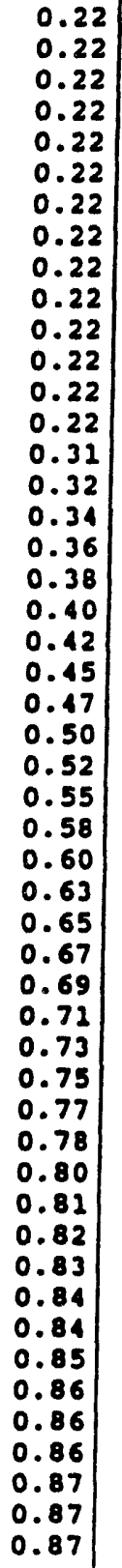 & $\begin{array}{l}\text { O.26 } \\
0.26 \\
0.26 \\
0.26 \\
0.26 \\
0.26 \\
0.26 \\
0.26 \\
0.26 \\
0.26 \\
0.26 \\
0.26 \\
0.26 \\
0.26 \\
0.26 \\
0.26 \\
0.27 \\
0 .\end{array}$ & $\begin{array}{l}0.30 \\
0.30 \\
0.30 \\
0.30 \\
0.30 \\
0.30 \\
0.30 \\
0.30 \\
0.30 \\
0.30 \\
0.30 \\
0.30 \\
0.30 \\
0.30 \\
0.30 \\
0.31 \\
0.36 \\
0.37 \\
0.38 \\
0.39 \\
0.41 \\
0.43 \\
0.44 \\
0.44 \\
0.46 \\
0.48 \\
0.50 \\
0.52 \\
0.55 \\
0.57 \\
0.60 \\
0.60 \\
0.62 \\
0.65 \\
0.67 \\
0.70 \\
0.72 \\
0.75 \\
0.76 \\
0.76 \\
0.78 \\
0.80 \\
0.90 \\
0.82 \\
0.80 \\
0.83 \\
0.84\end{array}$ \\
\hline
\end{tabular}


Table 4.3.2-4b

$\left(p_{1}=2 \mathrm{~atm}\right)$

Normalized Relative Velocity of Water at Vane Exit vs. Inlet Void Fraction for Various Homologous Flow Parameters at PI $=2$ atr DC Motor Case

\begin{tabular}{|c|c|c|c|c|c|c|}
\hline \multirow{2}{*}{$\begin{array}{l}\text { Inlet } \\
\text { Void } \\
\text { Fraction }\end{array}$} & \multicolumn{6}{|c|}{ Homologous Flow Parameter } \\
\hline & 0.4 & 0.6 & 0.8 & 1.0 & 1.2 & 1.4 \\
\hline $\begin{array}{l}0.00 \\
0.02 \\
0.04 \\
0.06 \\
0.08 \\
0.01 \\
0.10 \\
0.12\end{array}$ & $\begin{array}{l}0.09 \\
0.09 \\
0.09 \\
0.09 \\
0.09 \\
0.09 \\
0.09 \\
0.09 \\
0.09 \\
0.09 \\
0.09 \\
0.10 \\
0.70 \\
0.56 \\
0.59 \\
0 .\end{array}$ & $\begin{array}{l}0.13 \\
0.13 \\
0.13 \\
0.13 \\
0.13 \\
0.13 \\
0.13 \\
0.13 \\
0.13 \\
0.13 \\
0.14 \\
0.14 \\
0.36 \\
0.39 \\
0.42 \\
0.45 \\
0.48 \\
0.50 \\
0.53 \\
0.55 \\
0.58 \\
0.60 \\
0.62 \\
0.64 \\
0.65 \\
0.67 \\
0.69 \\
0.70 \\
0.72 \\
0.73 \\
0.74 \\
0.75 \\
0.76 \\
0.77 \\
0.78 \\
0.79 \\
0.80 \\
0.81 \\
0.81 \\
0.82 \\
0.82 \\
0.83 \\
0.83 \\
0.84 \\
0.84 \\
0.84 \\
0.84 \\
0.84 \\
0.84 \\
0.85\end{array}$ & 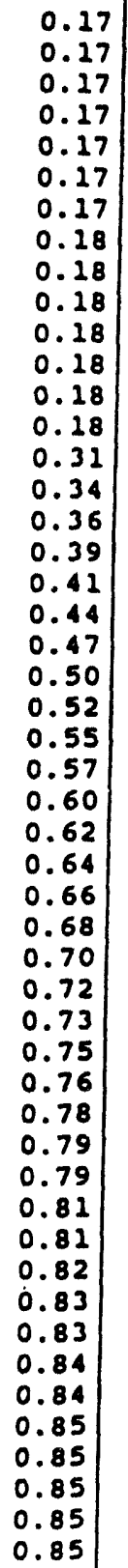 & 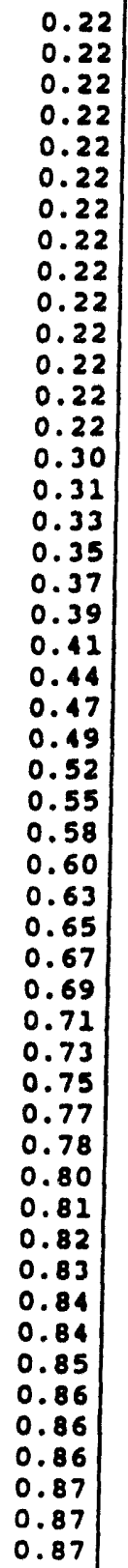 & 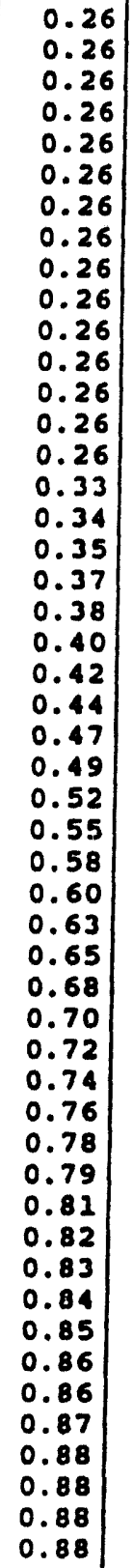 & 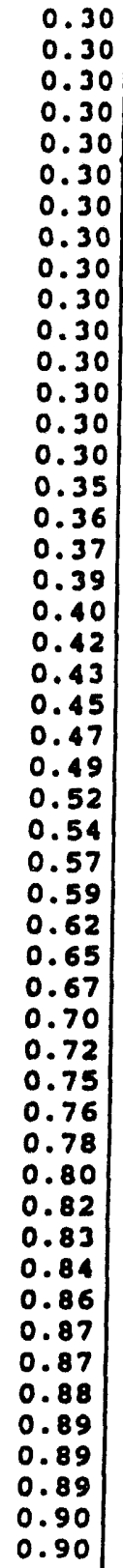 \\
\hline
\end{tabular}


Table 4.3.2-4c

$\left(p_{1}=1\right.$ atm $)$

Normalized Relative Velocity of Water at Vane Exit vs. Inlet Void Fraction for Various Homologous Flow Parameters at PI = 1 atm DC Motor Case

\begin{tabular}{|c|c|c|c|c|c|c|}
\hline \multirow{2}{*}{$\begin{array}{l}\text { Inlet } \\
\text { Vold } \\
\text { Fraction }\end{array}$} & \multicolumn{6}{|c|}{ Homologous Flow Parameter } \\
\hline & 0.4 & 0.6 & 0.8 & 1.0 & 1.2 & 1.4 \\
\hline $\begin{array}{l}\quad 0.00 \\
0.02 \\
0.04 \\
0.06 \\
0.08 \\
0.10\end{array}$ & $\begin{array}{l}\text { O. } 09 \\
0.09 \\
0.09 \\
0.09 \\
0.09 \\
0.09 \\
0.09 \\
0.09 \\
0.09 \\
0.09 \\
0.09 \\
0 .\end{array}$ & 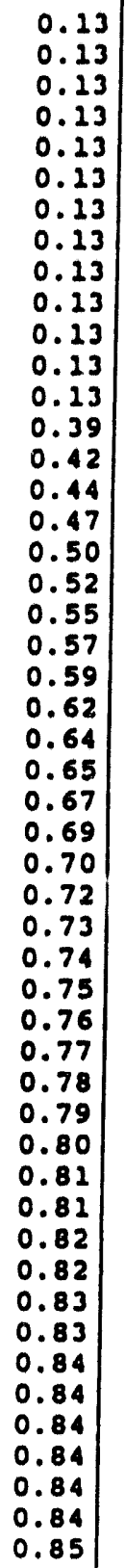 & 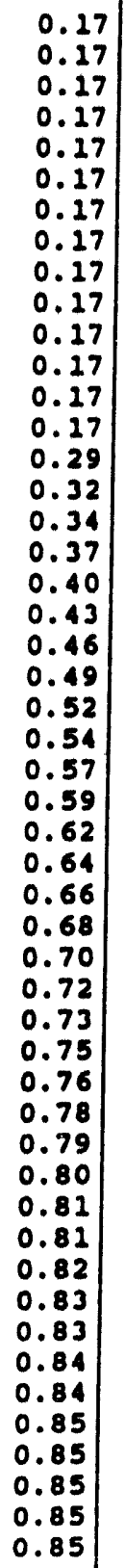 & $\begin{array}{l}0.22 \\
0.21 \\
0.21 \\
0.21 \\
0.21 \\
0.21 \\
0.21 \\
0.21 \\
0.21 \\
0.21 \\
0.21 \\
0.21 \\
0.21 \\
0.21 \\
0.21 \\
0.29 \\
0.31 \\
0.33 \\
0.35 \\
0.37 \\
0.37 \\
0.40 \\
0.42\end{array}$ & $\begin{array}{l}0.26 \\
0.26 \\
0.26 \\
0.26 \\
0.25 \\
0.25 \\
0.25 \\
0.25 \\
0.25 \\
0.25 \\
0.25 \\
0.25 \\
0.25 \\
0.25 \\
0.25 \\
0.25 \\
0.25 \\
0.31 \\
0.32 \\
0.32 \\
0.33 \\
0.34 \\
0.34 \\
0.36 \\
0.38 \\
0.40 \\
0.43 \\
0 .\end{array}$ & $\begin{array}{l}0.30 \\
0.30 \\
0.30 \\
0.30 \\
0.30 \\
0.30 \\
0.29 \\
0.29 \\
0.29 \\
0.29 \\
0.29 \\
0.29 \\
0.29 \\
0.29 \\
0.28 \\
0.33 \\
0.34 \\
0.35 \\
0.36 \\
0.37 \\
0.39 \\
0.42 \\
0.43 \\
0.45 \\
0.47 \\
0.50 \\
0.53 \\
0.55 \\
0.58 \\
0.61 \\
0.64 \\
0.67 \\
0.67 \\
0.79 \\
0.72 \\
0.74 \\
0.76 \\
0.89 \\
0.78 \\
0.90 \\
0.80 \\
0.82 \\
0.82 \\
0.83 \\
0.84 \\
0.86 \\
0.87\end{array}$ \\
\hline
\end{tabular}


Table 4.3.2-4d

$\left(p_{1}=0.05 \mathrm{~atm}\right)$

Normalized Relative Velocity of Water at Vane Exit vs. Inlet Vold Fraction tor Varlous Homologous Flow Parameters at Pl $=.05$ atm DC Motor Case

\begin{tabular}{|c|c|c|c|c|c|c|}
\hline \multirow{2}{*}{$\begin{array}{l}\text { Inlet } \\
\text { Vold } \\
\text { Fraction }\end{array}$} & \multicolumn{6}{|c|}{ Homologous Flow Parameter } \\
\hline & 0.4 & 0.6 & 0.8 & 2.0 & 2.2 & 1.4 \\
\hline $\begin{array}{l}0.00 \\
0.02 \\
0.04 \\
0.04 \\
0.06 \\
0.08 \\
0.10 \\
0.12 \\
0.14\end{array}$ & $\begin{array}{l}0.09 \\
0.08 \\
0.08 \\
0.08 \\
0.08 \\
0.08 \\
0.08 \\
0.08 \\
0.08 \\
0.08 \\
0.08 \\
0.08 \\
0.08 \\
0.58 \\
0.60 \\
0.62 \\
0.63 \\
0.65 \\
0.67 \\
0.68 \\
0.69 \\
0.70 \\
0.71 \\
0.72 \\
0.74 \\
0.75 \\
0.75 \\
0.76 \\
0.77 \\
0.78 \\
0.78 \\
0.79 \\
0.80 \\
0.80 \\
0.81 \\
0.81 \\
0.81 \\
0.82 \\
0.82 \\
0.83 \\
0.83 \\
0.0 \\
0.03 \\
0.83 \\
0.84 \\
0.84 \\
0.84 \\
0.84 \\
0.84 \\
0.84\end{array}$ & 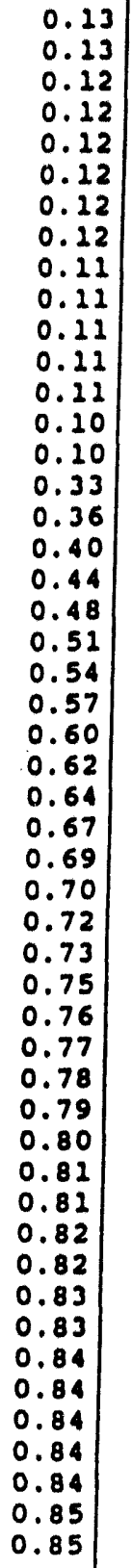 & 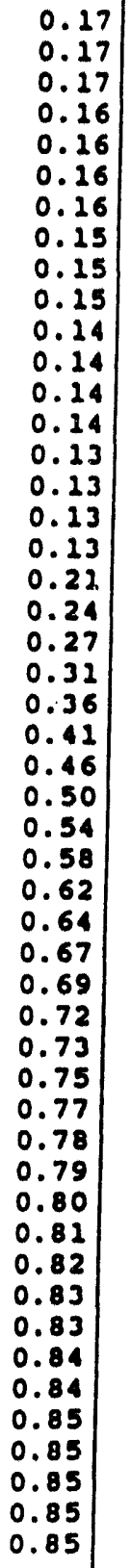 & $\begin{array}{l}\text { O.22 } \\
0.21 \\
0.21 \\
0.20 \\
0.20 \\
0.20 \\
0.20 \\
0.19 \\
0.19 \\
0.19 \\
0.28 \\
0.18 \\
0.18 \\
0.17 \\
0.27 \\
0.17 \\
0.16 \\
0.26 \\
0.16 \\
0.16 \\
0.15 \\
0.25 \\
0.25 \\
0.20 \\
0 .\end{array}$ & $\begin{array}{l}\text { O.26 } \\
0.25 \\
0.25 \\
0.24 \\
0.24 \\
0.24 \\
0.23 \\
0.23 \\
0.23 \\
0.22 \\
0.22 \\
0.21 \\
0.21 \\
0.21 \\
0.21 \\
0.20 \\
0.20 \\
0.19 \\
0.29 \\
0.19 \\
0.19 \\
0.18 \\
0.18 \\
0.17 \\
0 .\end{array}$ & $\begin{array}{l}0.30 \\
0.30 \\
0.29 \\
0.28 \\
0.28 \\
0.28 \\
0.27 \\
0.27 \\
0.26 \\
0.26 \\
0.25 \\
0.25 \\
0.24 \\
0.24 \\
0.23 \\
0.23 \\
0.22 \\
0.22 \\
0.21 \\
0.22 \\
0.20 \\
0.20 \\
0.29 \\
0.29 \\
0.29 \\
0.28 \\
0.23 \\
0.24 \\
0.27 \\
0.32 \\
0.10 \\
0.48 \\
0.55 \\
0.60 \\
0.66 \\
0.70 \\
0.74 \\
0.77 \\
0.80 \\
0.82 \\
0.84 \\
0.85 \\
0.86 \\
0.87 \\
0.88 \\
0.89 \\
0.89 \\
0.90 \\
0.90 \\
0.90\end{array}$ \\
\hline
\end{tabular}


Table 4.3.2-5n $\quad\left(p_{1}=3 \mathrm{~atm}\right)$

Normalized Rerative Velocity of Air at Vane Exit vs. Inlet Void
Fraction tor Various Homologous Flow Parameters at PI $=3$ atm
DC Motor Case

\begin{tabular}{|c|c|c|c|c|c|c|}
\hline \multirow{2}{*}{$\begin{array}{l}\text { Inlet } \\
\text { Vold } \\
\text { Fraction }\end{array}$} & \multicolumn{6}{|c|}{ Homologous Flow Parameter } \\
\hline & 0.4 & 0.6 & 0.8 & 1.0 & 2.2 & 1.4 \\
\hline $\begin{array}{l}\quad 0.00 \\
0.02 \\
0.04 \\
0.04 \\
0.08 \\
0.10\end{array}$ & 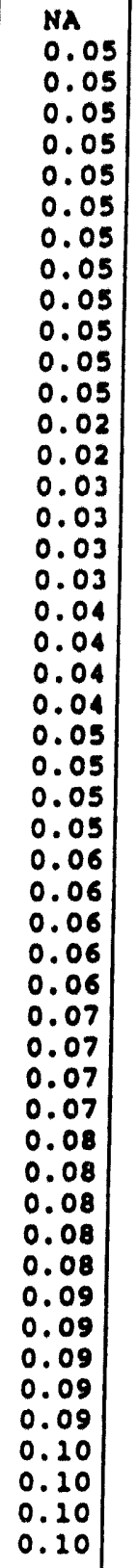 & 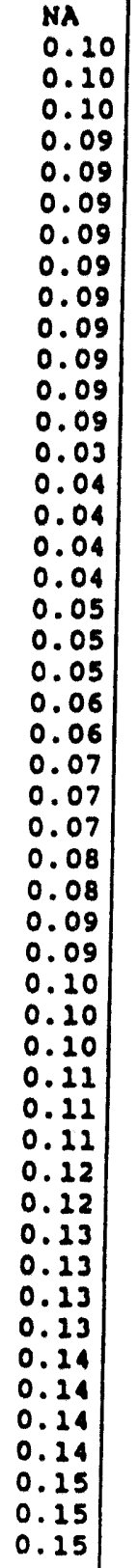 & $\begin{array}{l}\text { NA } \\
0.00 \\
0.15 \\
0.14 \\
0.14 \\
0.14 \\
0.14 \\
0.14 \\
0.14 \\
0.14 \\
0.13 \\
0.13 \\
0.13 \\
0.13 \\
0.13 \\
0.13 \\
0.07 \\
0.07 \\
0.07 \\
0.07 \\
0.07 \\
0.07 \\
0.07 \\
0.07 \\
0.07 \\
0.07 \\
0.08 \\
0.08 \\
0.09 \\
0.09 \\
0.10 \\
0.10 \\
0.10 \\
0.21 \\
0.12\end{array}$ & 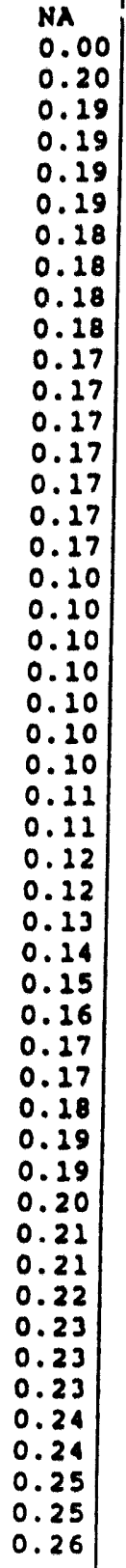 &  &  \\
\hline
\end{tabular}


Table 4.3.2-5b $\quad\left(p_{1}=2\right.$ atm $)$

Normalized Rerative Velocity of Air at Vane Exit vs. Inlet Void
Fraction for Various Homologous Flow Parameters at PI 2 atm
DC Motor Case

\begin{tabular}{|c|c|c|c|c|c|c|}
\hline \multirow{2}{*}{$\begin{array}{l}\text { Inlet } \\
\text { Vold } \\
\text { Fraction }\end{array}$} & \multicolumn{6}{|c|}{ Homologous Flow Parameter } \\
\hline & 0.4 & 0.6 & 0.8 & 1.0 & 2.2 & 1.4 \\
\hline $\begin{array}{l}\quad 0.00 \\
0.02 \\
0.04 \\
0.06 \\
0.08 \\
0.10 \\
0.12 \\
0.14 \\
0.16 \\
0.18\end{array}$ & $\begin{array}{l}\text { NA } \\
0.02 \\
0.04 \\
0.04 \\
0.04 \\
0.04 \\
0.04 \\
0.04 \\
0.05 \\
0.05 \\
0.05 \\
0.05 \\
0.02 \\
0.02 \\
0.02 \\
0.02 \\
0.02 \\
0.03 \\
0.03 \\
0.03 \\
0.03 \\
0.03 \\
0.03 \\
0.03 \\
0.03 \\
0.04 \\
0.04 \\
0.04\end{array}$ & 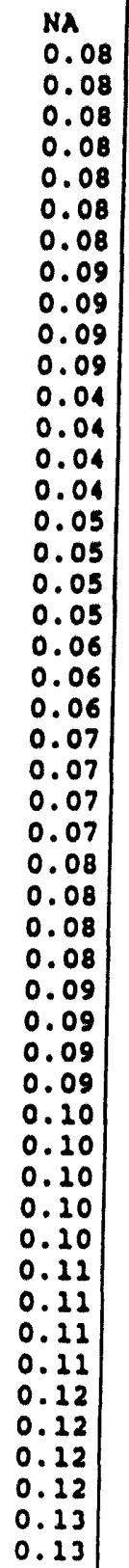 & 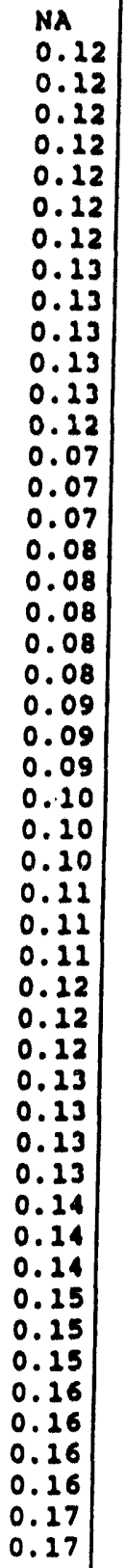 &  & 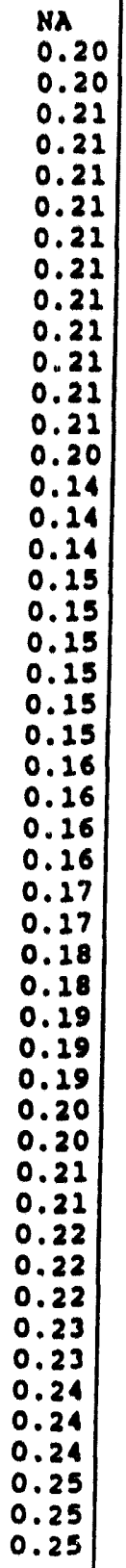 & 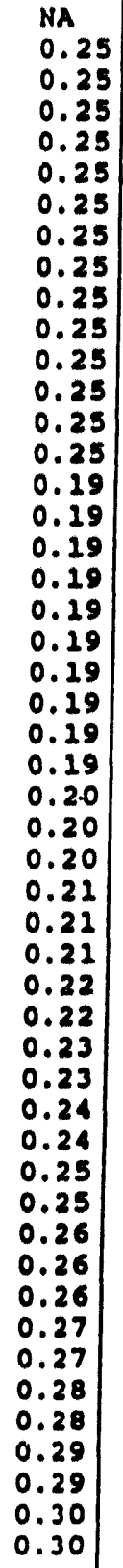 \\
\hline
\end{tabular}


Table 4.3.2-5c $\quad\left(p_{1}=1 \mathrm{~atm}\right)$

\begin{tabular}{|c|c|c|c|c|c|c|}
\hline \multirow{2}{*}{$\begin{array}{l}\text { Inlet } \\
\text { Vold } \\
\text { Fraction }\end{array}$} & \multicolumn{6}{|c|}{ Homologous Flow Parameter } \\
\hline & 0.4 & 0.6 & 0.8 & 1.0 & 1.2 & 1.4 \\
\hline $\begin{array}{l}0.00 \\
0.02 \\
0.04 \\
0.06 \\
0.08 \\
0.10 \\
0.22 \\
0.14 \\
0.16 \\
0.18 \\
0.20 \\
0.22 \\
0.24 \\
0.26 \\
0.28 \\
0.30 \\
0.32 \\
0.34 \\
0.36 \\
0.38 \\
0.40 \\
0.42\end{array}$ & $\begin{array}{l}\text { NA } \\
0.03 \\
0.04 \\
0.04 \\
0.04 \\
0.04 \\
0.04 \\
0.04 \\
0.04 \\
0.04 \\
0.05 \\
0.02 \\
0.02 \\
0.02 \\
0.03 \\
0.03 \\
0.03 \\
0.03 \\
0.03 \\
0.03 \\
0.04 \\
0.04 \\
0.04 \\
0.04 \\
0.04 \\
0.05 \\
0.05 \\
0.05 \\
0.05 \\
0.05 \\
0.05 \\
0.05 \\
0.06 \\
0.06 \\
0.06 \\
0.06 \\
0.06 \\
0.06 \\
0.07 \\
0.07 \\
0.07 \\
0.07 \\
0.07 \\
0.07 \\
0.08 \\
0.08 \\
0.08 \\
0.08 \\
0.08 \\
0.08 \\
0.08\end{array}$ & $\begin{array}{l}\text { NA } \\
0.08 \\
0.08 \\
0.08 \\
0.08 \\
0.08 \\
0.08 \\
0.08 \\
0.08 \\
0.08 \\
0.08 \\
0.08 \\
0.08 \\
0.04 \\
0.04 \\
0.04 \\
0.04 \\
0.04 \\
0.05 \\
0.05 \\
0.05 \\
0.05 \\
0.05 \\
0.06 \\
0.06 \\
0.06 \\
0.07 \\
0.07 \\
0.07 \\
0.07 \\
0.07 \\
0.08 \\
0.08 \\
0.08 \\
0.08 \\
0.08 \\
0.09 \\
0.09 \\
0.09 \\
0.09 \\
0.10\end{array}$ & $\begin{array}{l}\text { NA } \\
0.12 \\
0.12 \\
0.12 \\
0.12 \\
0.12 \\
0.12 \\
0.12 \\
0.12 \\
0.12 \\
0.12 \\
0.12 \\
0.12 \\
0.12 \\
0.06 \\
0.06 \\
0.07 \\
0.07 \\
0.07 \\
0.07 \\
0.08 \\
0.08 \\
0.08 \\
0.09 \\
0.09 \\
0.09 \\
0.10 \\
0.10 \\
0.10 \\
0.11 \\
0.11 \\
0.12 \\
0.12 \\
0.12 \\
0.13 \\
0.13 \\
0.13 \\
0.13 \\
0.14 \\
0.14 \\
0.11 \\
0.15 \\
0.15 \\
0.15 \\
0.16 \\
0.16 \\
0.16 \\
0.16 \\
0.17 \\
0.17\end{array}$ & 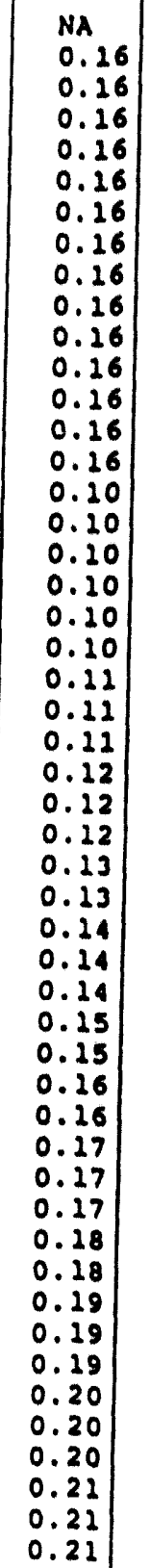 & $\begin{array}{l}N A \\
0.20 \\
0.20 \\
0.20 \\
0.20 \\
0.20 \\
0.20 \\
0.20 \\
0.20 \\
0.20 \\
0.20 \\
0.20 \\
0.20 \\
0.20 \\
0.20 \\
0.23 \\
0.13 \\
0.13 \\
0.13 \\
0.14 \\
0.14 \\
0.14 \\
0.24 \\
0.14 \\
0.14 \\
0.15 \\
0.15 \\
0.16 \\
0.16 \\
0.17 \\
0.17 \\
0.18 \\
0.28 \\
0.19 \\
0.19 \\
0.20 \\
0.20 \\
0.21 \\
0.21 \\
0.21 \\
0.22 \\
0.22 \\
0.23 \\
0.23 \\
0.24 \\
0.24 \\
0.24 \\
0.25 \\
0.25 \\
0.25\end{array}$ & 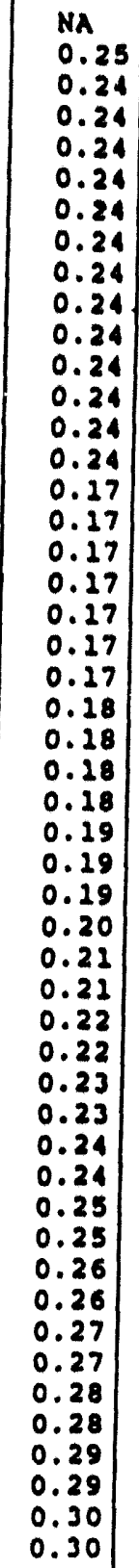 \\
\hline
\end{tabular}




\begin{abstract}
Normalized Rerative Velocity of Alr at Vane Exit va. Indet Vold Fraction for Varlous Homologous Flow Parameters at Pl $=0.05$ atm DC Motor Case
\end{abstract}

\begin{tabular}{|c|c|c|c|c|c|c|}
\hline \multirow{2}{*}{$\begin{array}{l}\text { Inlet } \\
\text { Vold } \\
\text { Fraction }\end{array}$} & \multicolumn{6}{|c|}{ Homologous Flow Parameter } \\
\hline & 0.4 & 0.6 & 0.8 & 2.0 & 1.2 & 2.4 \\
\hline $\begin{array}{l}0.00 \\
0.02 \\
0.04 \\
0.06 \\
0.08 \\
0.10 \\
0.12 \\
0.14 \\
0.16 \\
0.18 \\
0.20 \\
0.22\end{array}$ & $\begin{array}{l}\text { NA } \\
0.00 \\
0.03 \\
0.03 \\
0.02 \\
0.02 \\
0.02 \\
0.02 \\
0.02 \\
0.02 \\
0.02 \\
0.02 \\
0.02 \\
0.01 \\
0.02 \\
0.02 \\
0.02 \\
0.02 \\
0.02 \\
0.02 \\
0.02 \\
0.03 \\
0.03 \\
0.03 \\
0.03 \\
0.03 \\
0.03 \\
0.04 \\
0.04 \\
0.04 \\
0.04 \\
0.05 \\
0.05 \\
0.03 \\
0.03 \\
0.06 \\
0.06 \\
0.06 \\
0.06 \\
0.07 \\
0.07 \\
0.07 \\
0.07 \\
0.07 \\
0.08\end{array}$ & $\begin{array}{l}\text { NA } \\
0.00 \\
0.00 \\
0.06 \\
0.06 \\
0.06 \\
0.06 \\
0.06 \\
0.05 \\
0.05 \\
0.05 \\
0.05 \\
0.03 \\
0.03 \\
0.03 \\
0.01 \\
0.01 \\
0.01 \\
0.02 \\
0.02 \\
0.02 \\
0.02 \\
0.02 \\
0.03 \\
0.03 \\
0.03 \\
0.04 \\
0.04 \\
0.04 \\
0.05 \\
0.05 \\
0.05 \\
0.06 \\
0.06 \\
0.07 \\
0.07 \\
0.08 \\
0.08 \\
0.09 \\
0.09 \\
0.10 \\
0.10\end{array}$ & $\begin{array}{l}\text { NA } \\
0.00 \\
0.00 \\
0.10 \\
0.10 \\
0.20 \\
0.09 \\
0.09 \\
0.09 \\
0.09 \\
0.09 \\
0.08 \\
0.08 \\
0.08 \\
0.08 \\
0.08 \\
0.08 \\
0.07 \\
0.07 \\
0.07 \\
0.07 \\
0.02 \\
0.02 \\
0.02 \\
0.02 \\
0.02 \\
0.03 \\
0.03 \\
0.03 \\
0.03 \\
0.03 \\
0.04 \\
0.04 \\
0.05\end{array}$ & $\begin{array}{l}\text { NA } \\
0.00 \\
0.00 \\
0.00 \\
0.14 \\
0.13 \\
0.23 \\
0.13 \\
0.13 \\
0.22 \\
0.12 \\
0.12 \\
0.12 \\
0.12 \\
0.12 \\
0.20 \\
0.10 \\
0.10 \\
0.20 \\
0.09 \\
0.09 \\
0.04 \\
0.04 \\
0.03 \\
0.03 \\
0.04 \\
0.04 \\
0.04 \\
0.05 \\
0.06 \\
0.06 \\
0.07 \\
0.08 \\
0.09 \\
0.10 \\
0.21 \\
0.22 \\
0.13 \\
0.14 \\
0.25 \\
0.16 \\
0.27 \\
0.18 \\
0.19 \\
0.29 \\
0.20 \\
0.20 \\
0.21 \\
0.21 \\
0.21\end{array}$ & 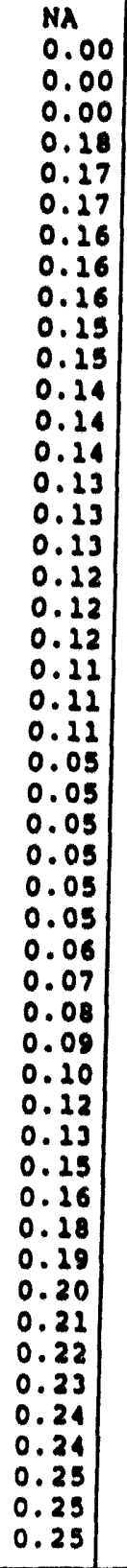 & 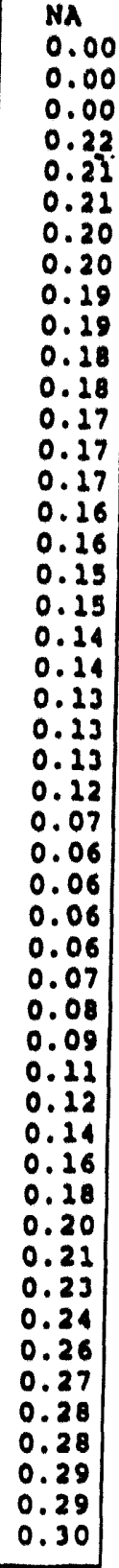 \\
\hline
\end{tabular}


Table 4.3.2-2a Homologous Torque at $p_{1}=3 \mathrm{~atm}$ (DC Motor)

Homologous Flow Parameter $v / \alpha_{N}$

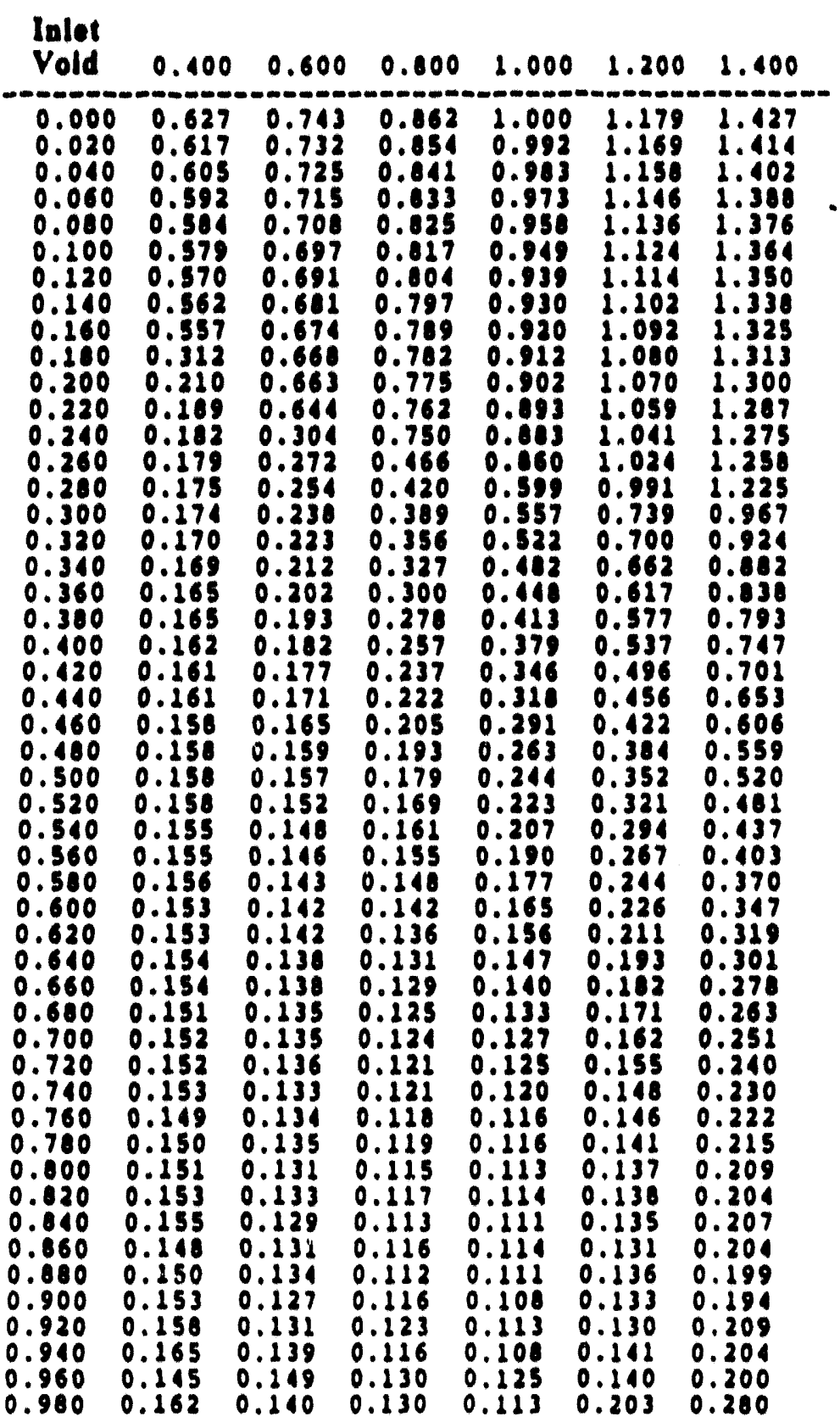









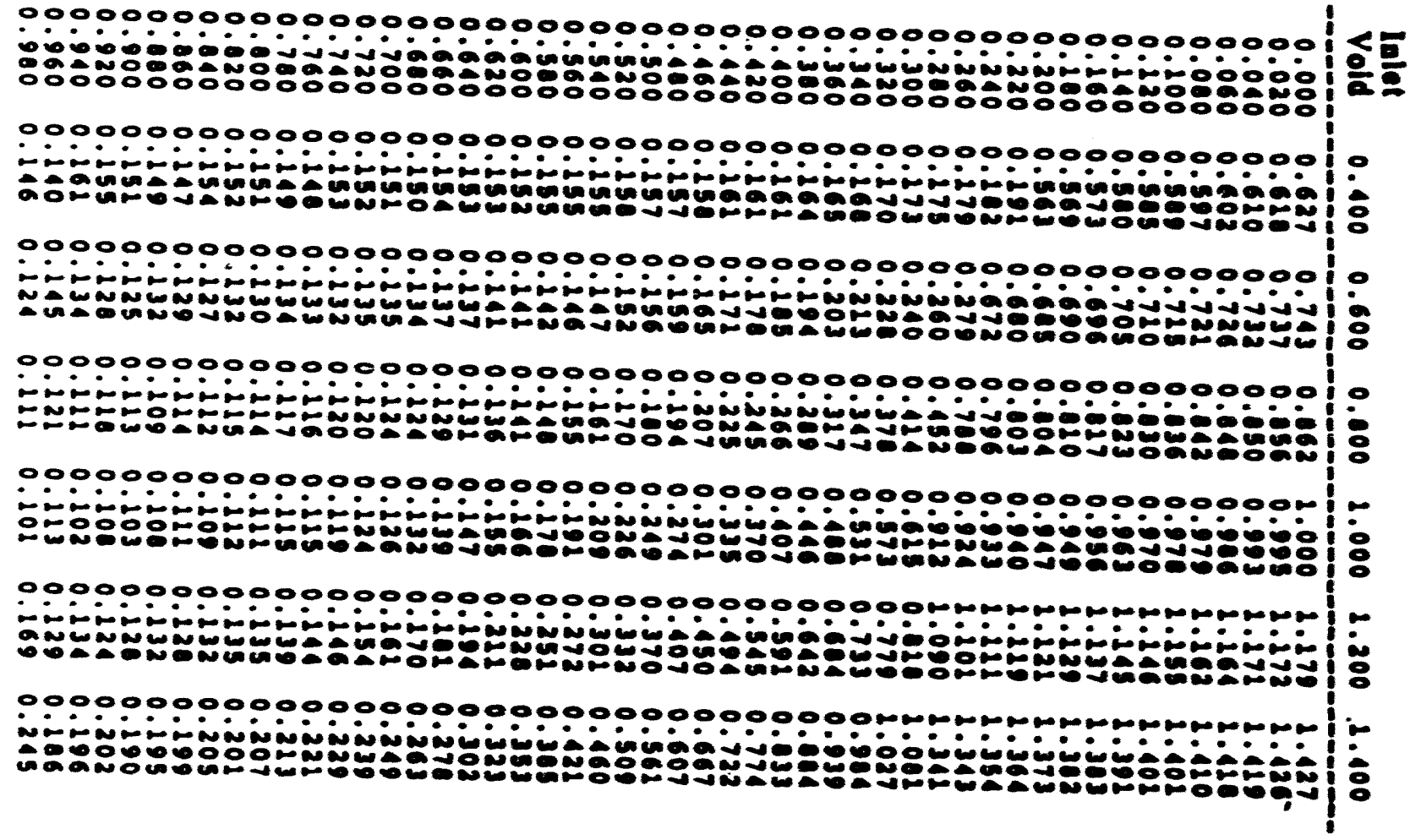


00000000000000000000000000000000000000000000000000

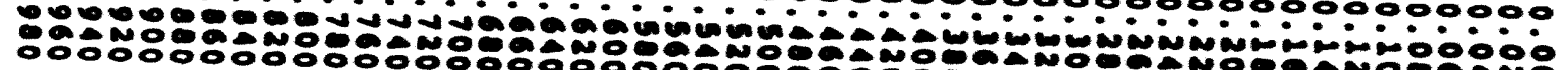
 1000000000000000000000000000000000000000000000000 јабй́ 00000000000000000000000000000000000000000000000000

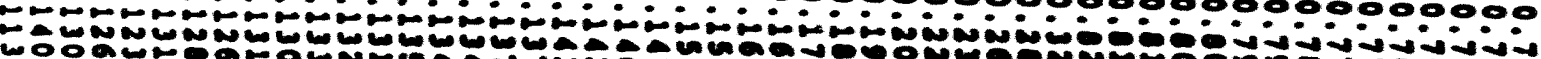

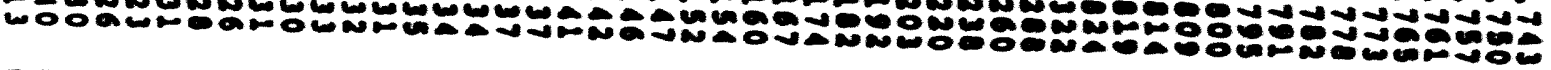
00000000000000000000000000000000000000000000000000

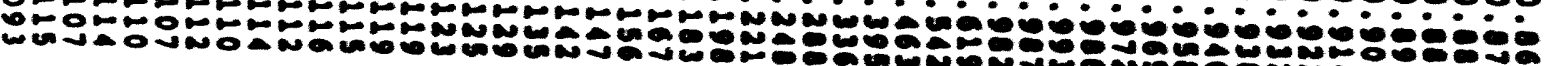
0000000000000000000000000000050 מ.

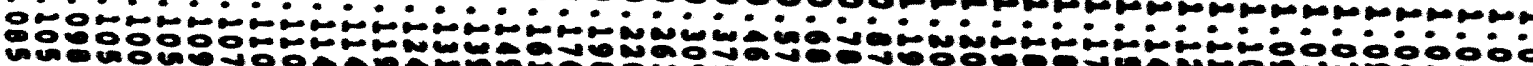

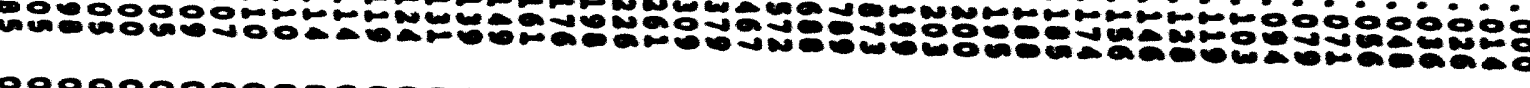



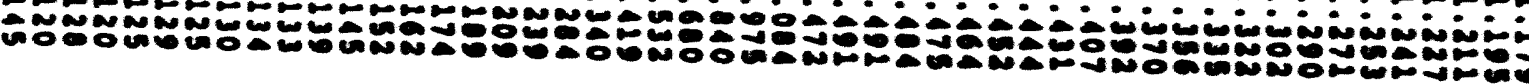

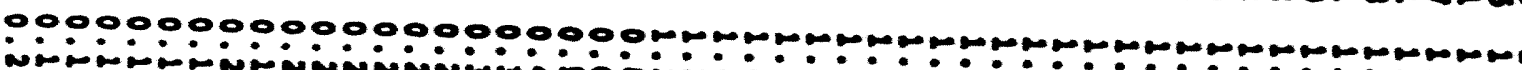

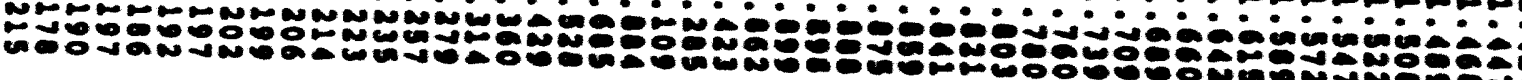


Table 4.5.2-1a $\quad\left(p_{1}=3\right.$ atm)

Siafic Pressure Increase, $\left(p_{2}-p_{1}\right)(p s i)$

Inlet Vold

Fractlon

$\begin{array}{ll}0.1 & 0.2 \\ 0.00 & 22.589 \\ 0.02 & 0.745 \\ 0.04 & 0.586 \\ 0.06 & 0.612 \\ 0.08 & 0.743 \\ 0.10 & 0.899 \\ 0.12 & 0.998 \\ 0.14 & 0.959 \\ 0.16 & 0.469 \\ 0.18 & 0.398 \\ 0.20 & 0.356 \\ 0.22 & 0.324 \\ 0.24 & 0.296 \\ 0.26 & 0.274 \\ 0.28 & 0.251 \\ 0.30 & 0.231 \\ 0.32 & 0.215 \\ 0.34 & 0.200 \\ 0.36 & 0.186 \\ 0.38 & 0.174 \\ 0.40 & 0.163 \\ 0.42 & 0.152 \\ 0.44 & 0.142 \\ 0.46 & 0.133 \\ 0.48 & 0.125 \\ 0.50 & 0.117 \\ 0.52 & 0.110 \\ 0.54 & 0.104 \\ 0.56 & 0.098 \\ 0.58 & 0.092 \\ 0.60 & 0.087 \\ 0.62 & 0.082 \\ 0.64 & 0.078 \\ 0.66 & 0.074 \\ 0.68 & 0.071 \\ 0.70 & 0.067 \\ 0.72 & 0.064 \\ 0.74 & 0.062 \\ 0.76 & 0.059 \\ 0.78 & 0.056 \\ 0.80 & 0.054 \\ 0.82 & 0.052 \\ 0.84 & 0.050 \\ 0.86 & 0.049 \\ 0.88 & 0.047 \\ 0.90 & 0.045 \\ 0.92 & 0.044 \\ 0.94 & 0.043 \\ 0.96 & 0.041 \\ 0.98 & 040 \\ & \end{array}$

0.4

22.357

21.740

21.140

20.550

19.979

19.425

28.883

28.353

27.833

17.256

4.249

2.324

1.859

1.618

1.419

1.250

1.104

0.977

0.868

0.770

0.684

0.607

0.539

0.479

0.424

0.378

0.335

0.296

0.263

0.233

0.206

0.183

0.162

0.145

0.128

0.124

0.103

0.092

0.083

0.075

0.068

0.064

0.059

0.055

0.051

0.048

0.046

0.044

0.042

0.040
Homologous Paramecer

$\begin{array}{llllll}0.6 & 0.8 & 1.0 & 1.2 & 1.4\end{array}$

$\begin{array}{lll}22.131 & 21.911 & 21.693\end{array}$

$21.627 \quad 21.459 \quad 21.262$

21.228

21.009

20.633

20.559

20.141

20.109

19.653

29.657

20.846

20.426

20.004

19.168

19.205

29.578

18.686

18.753

19.150

18.205

18.299

18.719

18.287

17.728

27.253

26.704

17.846

17.852

17.390

16.936

7.212

5.503

16.406

11.365

4.730

9.042

4.081

8.006

17.414

16.974

16.519

15.950

11.539

10.492

3.525

3.046

2.633

7.046

6.163

5.361

2.276

1.967

4.640

4.001

1.700

1.469

1.268

1.094

0.942

0.811

0.697

0.598

0.512

0.437

0.373

0.317

0.268

0.228

0.193

0.163

3.443

2.948

2.518

2.144

1.822

1.543

1.303

1.098

0.922

0.770

0.642

0.531

0.438

0.359

0.293

0.238

0.238

0.192

0.255

0.123

0.099

0.100

0.081

0.075

0.065

0.067

9.557

8.635

7.736

6.870

6.046

5.278

4.574

3.943

3.371

2.864

2.420

2.034

1.700

1.412

1.167

0.959

0.781

0.632

0.507

0.403

0.316

0.245

0.188

0.142

0.107

0.079

0.060

0.058

0.056

0.045

0.053

0.049

0.049

0.037

0.044

0.032

0.046

0.040

0.031

0.044

0.039

0.039

0.032

0.035

0.041

0.038
21.478

21.067

20.681

20.291

19.896

19.495

19.089

18.677

18.262

17.842

17.418

I6. 992

16.557

16.064

15.441

11.676

10.833

9.993

9.150

8.309

7.477

6.666

5.886

5. 151

4.475

3.842

3.266

2.754

2. 303

1.910

2.571

1.280

1.033

0.825

0.649

0.505

0.386

0.289

0.210

0.149

0.102

0.067

0.042

0.026

0.027

0.024

0.015

0.020

0.026

0.035
21.264

20.874

20.511

20.143

19.769

19.390

19.005

18.614

18.219

17.829

17.412

17.003

16.587

16.136

15.572

12.298

11.502

20.724

9.939

9.146

8.350

7.557

6.776

6.021

5.304

4.610

3.960

3.364

2.825

2. 348

1.929

1.566

1.255

0.991

0.770

0.585

0.435

0.313

0.216

0.139 
$\underset{\text { (contlaued) }}{4.5 .2-1 \mathrm{a}} \quad\left(\mathrm{P}_{1}=3 \mathrm{~atm}\right)$

$r_{12}$ Liquid Flow Velocily (fect/sec)

Inlet Void

Fraction

$\begin{array}{ll}01 & 0.2 \\ 0.00 & 2.251 \\ 0.02 & 2.253 \\ 0.04 & 2.252 \\ 0.06 & 2.246 \\ 0.08 & 2.237 \\ 0.10 & 2.221 \\ 0.12 & 2.215 \\ 0.14 & 2.205 \\ 0.16 & 2.200 \\ 0.18 & 2.200 \\ 0.20 & 2.190 \\ 0.22 & 2.180 \\ 0.24 & 2.171 \\ 0.26 & 2.172 \\ 0.28 & 2.163 \\ 0.30 & 2.150 \\ 0.32 & 2.151 \\ 0.34 & 2.146 \\ 0.36 & 2.140 \\ 0.38 & 2.134 \\ 0.40 & 2.128 \\ 0.42 & 2.121 \\ 0.44 & 2.115 \\ 0.46 & 2.109 \\ 0.48 & 2.102 \\ 0.50 & 2.096 \\ 0.52 & 2.089 \\ 0.54 & 2.083 \\ 0.56 & 2.077 \\ 0.58 & 2.071 \\ 0.60 & 2.065 \\ 0.62 & 2.059 \\ 0.64 & 2.054 \\ 0.66 & 2.048 \\ 0.68 & 2.043 \\ 0.70 & 2.037 \\ 0.72 & 2.031 \\ 0.74 & 2.026 \\ 0.76 & 2.020 \\ 0.78 & 2.015 \\ 0.80 & 2.009 \\ 0.82 & 2.003 \\ 0.84 & 1.998 \\ 0.86 & 1.992 \\ 0.88 & 1.986 \\ 0.90 & 1.981 \\ 0.92 & 1.975 \\ 0.94 & 1.954 \\ 0.96 & \\ 0.98 & \\ 0.95 \\ 0.95\end{array}$

Homologous Paramcter

\begin{tabular}{|c|c|c|c|c|c|}
\hline 0.4 & 0.6 & 0.8 & 1.0 & 1.2 & 1.4 \\
\hline $\begin{array}{l}4.503 \\
4.487 \\
4.476 \\
4.466 \\
4.457 \\
4.450 \\
4.445 \\
4.440 \\
4.435 \\
4.431 \\
4.371 \\
4.378 \\
4.371 \\
4.360 \\
4.348 \\
4.336 \\
4.324 \\
4.311 \\
.298 \\
1.285 \\
1.272 \\
1.259 \\
1.245 \\
1.232 \\
1.218 \\
.205 \\
.192 \\
.179 \\
.166 \\
.153 \\
.140 \\
.128 \\
.115 \\
.103\end{array}$ & $\begin{array}{l}6.754 \\
6.738 \\
6.721 \\
6.704 \\
6.689 \\
6.674 \\
6.660 \\
6.648 \\
6.638 \\
6.629 \\
6.623 \\
6.620 \\
6.554 \\
6.590 \\
6.595 \\
6.589 \\
6.538 \\
6.492 \\
6.473 \\
6.456 \\
6.439 \\
6.422 \\
6.404 \\
6.385 \\
6.365 \\
6.344 \\
6.323 \\
6.301 \\
6.279 \\
6.257 \\
6.235 \\
6.213 \\
6.191 \\
6.170 \\
6.249 \\
6.128 \\
5.107 \\
5.087 \\
5.067 \\
5.048 \\
5.029 \\
5.010 \\
. .991 \\
.973 \\
.954 \\
.936\end{array}$ & 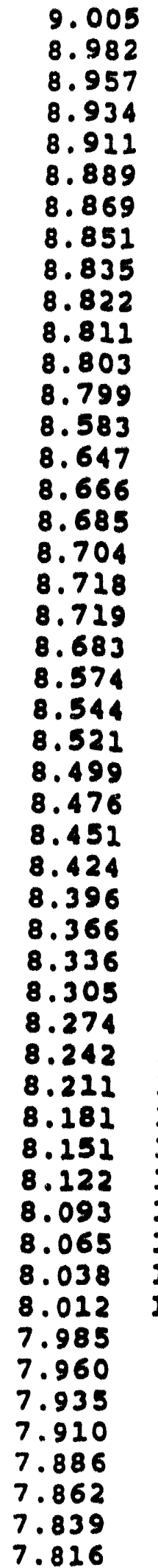 & 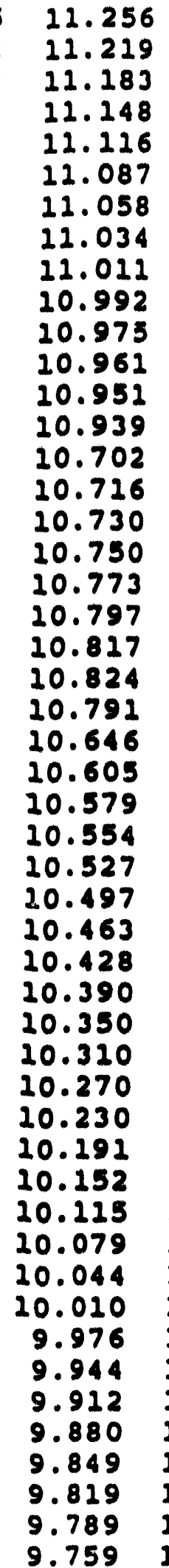 & $\begin{array}{l}13.508 \\
13.429 \\
13.352 \\
13.277 \\
13.206 \\
13.144 \\
13.096 \\
13.055 \\
13.021 \\
12.992 \\
12.969 \\
12.951 \\
12.938 \\
12.910 \\
12.799 \\
12.806 \\
12.811 \\
12.822 \\
12.839 \\
12.860 \\
12.882 \\
12.901 \\
12.904 \\
12.851 \\
12 \\
12.690 \\
12.650 \\
12.624 \\
12.59 \\
12\end{array}$ & $\begin{array}{l}60 \\
43 \\
17 \\
86 \\
48 \\
05 \\
57 \\
04 \\
50 \\
95 \\
40 \\
87 \\
36 \\
87 \\
40 \\
94 \\
49 \\
05\end{array}$ \\
\hline
\end{tabular}


Table 4.5.2-1a ( $\left.\quad p_{1}=3 \mathrm{~atm}\right)$ (continued)

$v_{c^{2}}$ Gas Flow Velocity (rect/sec)

Inlet Void

Fraction

$\begin{array}{ll}01 & 0.2 \\ 0.00 & 0.000 \\ 0.02 & 2.048 \\ 0.04 & 2.144 \\ 0.06 & 2.249 \\ 0.08 & 2.343 \\ 0.10 & 2.420 \\ 0.12 & 2.437 \\ 0.14 & 2.461 \\ 0.16 & 2.493 \\ 0.18 & 2.520 \\ 0.20 & 2.516 \\ 0.22 & 2.512 \\ 0.24 & 2.510 \\ 0.26 & 2.517 \\ 0.28 & 2.510 \\ 0.30 & 2.497 \\ 0.32 & 2.497 \\ 0.34 & 2.490 \\ 0.36 & 2.483 \\ 0.38 & .2 .475 \\ 0.40 & 2.468 \\ 0.42 & 2.460 \\ 0.44 & 2.453 \\ 0.46 & 2.446 \\ 0.48 & 2.438 \\ 0.50 & 2.431 \\ 0.52 & 2.424 \\ 0.54 & 2.416 \\ 0.56 & 2.409 \\ 0.58 & 2.401 \\ 0.60 & 2.394 \\ 0.62 & 2.386 \\ 0.64 & 2.378 \\ 0.66 & 2.370 \\ 0.68 & 2.363 \\ 0.70 & 2.355 \\ 0.72 & 2.348 \\ 0.74 & 2.341 \\ 0.76 & 2.333 \\ 0.78 & 2.326 \\ 0.80 & 2.319 \\ 0.82 & 2.312 \\ 0.84 & 2.305 \\ 0.86 & 2.297 \\ 0.88 & 2.290 \\ 0.90 & 2.283 \\ 0.92 & 2.276 \\ 0.94 & 2.269 \\ 0.96 & 2.253 \\ 0.98 & \\ 0.95 \\ 0.96\end{array}$

Homologous Parameter$$
0.4
$$

0.000

3.424

3.429

3.433

3.437

3.441

3.445

3.448

3.451

3.453

4.550

4.693

4.726

4.746

4.762

4.775

4.785

4.793

4.799

4.803

4.805

4.806

4.805

4.802

4.799

4.794

4.787

4.780

4.772

4.763

4.754

4.743

4.733

4.721

4.710

4.698

4.685

4.673

4.660

4.647

4.633

4.620

4.607

4.593

4.580

4.566

4.552

4.538

4. 525

4.511
0.6

0.000

4.724

4.761

4.791

4.815

4.835

4.852

4.866

4.877

4.888

4.897

4.910

6.163

6.254

6.321

6.407

6.584

6.729

6.797

6.850

6.894

6.931

6.962

6.989

7.011

7.028

7.042

7.052

7.059

7.062

7.062

7.059

7.054

7.046

7.036

7.025

7.011

6.997

6.981

6.964

6.947

6.928

6.909

6.890

6.870

6.850

6.829

6.809

6.788

6.767
0.8

0.000

6.515

6.550

6.579

6.602

6.622

6.638

6.650

6.661

6.670

6.679

6.686

6.700

7.981

8.072

8.122

8.181

8.251

8.334

8.435

8.587

8.837

8.942

9.022

9.089

9.146

9.195

9.236

9.270

9.297

9.317

9.332

9.340

9.344

9.343

9.337

9.327

9.314

9.299

9.280

9.260

9.237

9.214

9.188

9.162

9.135

9.108

9.080

9.052

9.024
1.0

0.000

8.671

8.652

8.650

8.637

8.627

8.626

8. 618

8.617

8.611

8.611

8.611

8.612

8.648

9.976

10.011

10.052

10.105

10.171

10.251

10.347

10.466

10.635

10.939

11.080

11.187

11.279

11.358

11.427

11.486

11.534

11.573

11.603

11.623

11.636

11.641

11.638

11.630

11.616

11.598

11.575

11.550

11.521

11.491

11.458

11.425

11.390

11.354

11.318

11.281
1.2

1.4

0.000

12.462

12.392

12.324

12.258

12.104

11.897

11.710

11.549

12.411

11.293

$-11.192$

11.107

11.106

11.369

11.999

12.029

12.070

12.125

12.195

12.282

12.389

12.527

12.736

13.066

13.225

13.355

13.469

13.571

13.661

13.739

13.805

13.858

13.898

13.927

13.945

13.952

13.949

13.939

13.921

23.897

13.868

13.835

13.798

13.759

13.717

13.674

13.630

13.585

13.539

0.000

14.627

14.546

14.468

14.393

14.321

14.251

14.184

14.120

14.060

14.002

13.948

13.898

13.853

13.821

14.057

14.083

14.119

14.169

14.235

14.321

14.430 
Table 4.5.2-1a $\quad\left(p_{1}=3 \mathrm{~atm}\right)$

(continued)

$\alpha_{2}$ Void Fraction

Inlet Void

Fraction

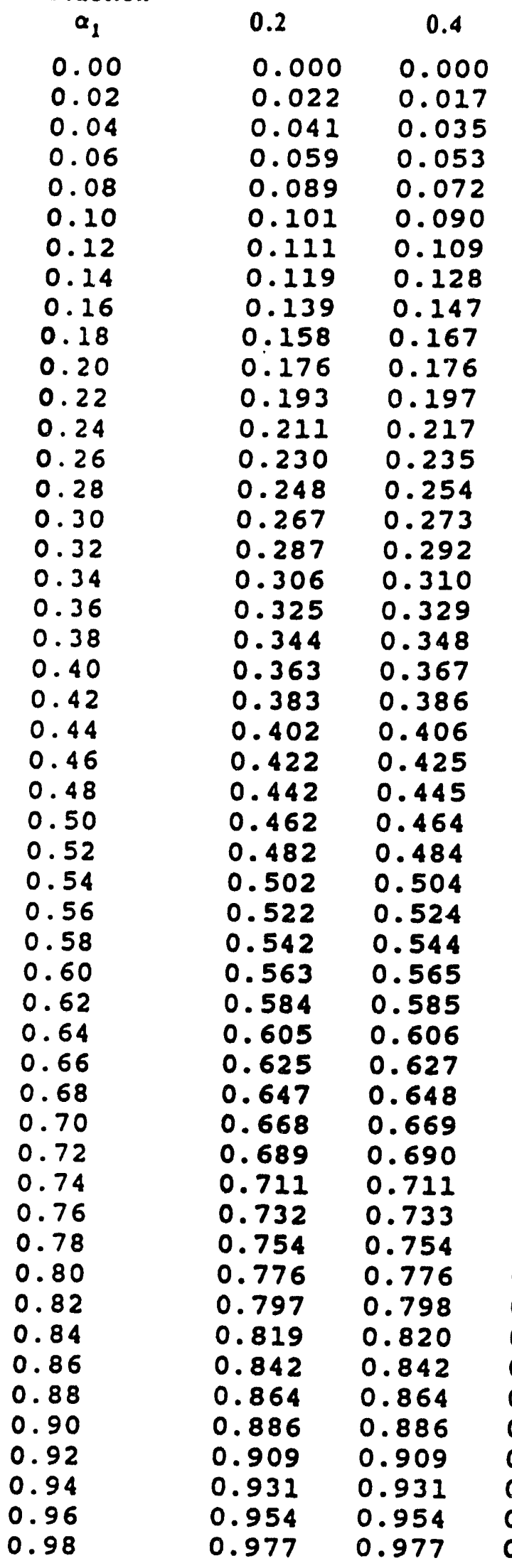

Homologous Parameler

0.6

0.8
0.000

0.018

0.017

0.035

0.035

0.053

0.052

0.071

0.089

0.108

0.126

0.145

0.165

0.184

0.204

0.218

0.242

0.070

0.088

0.106

0.125

0.144

0.163

0.182

0.202

0.222

0.223

0.263

0.283

0.250

0.272

0.298

0.295

0.314

0.333

0.352

0.371

0.390

0.410

0.429

0.448

0.468

0.487

0.507

0.527

0.547

0.567

0.587

0.607

0.317

0.339

0.360

0.378

0.391

0.410

0.429

0.449

0.469

0.489

0.508

0.528

0.548

0.568

0.588

0.608

0.628

0.629

0.648

0.649

0.670

0.669

0.691

0.712

0.733

0.712

0.733

0.754

0.776

0.798

0.820

0.842

0.864

0.886

0.909

0.931

0.954

0.977

1.0
0.000
0.017
0.034
0.051
0.068
0.086
0.104
0.123
0.141
0.160
0.179
0.199
0.219
0.239
0.242
0.264
0.286
0.309
0.331
0.353

0.353

0.375

0.397

0.416

0.429

0.448

0.468

0.488

0.508

0.528

0.548

0.568

0.588

0.608

0.629

0.649

0.670

0.691

0.712

0.733

0.754

0.776

0.798

0.819

0.842

0.864

0.886

0.909

0.931

0.954

0.977
1.2

0.000

0.014

0.029

0.044

0.059

0.075

0.092

0.110

0.129

0.147

0.167

.0 .186

0.207

0.226

0.240

0.262

0.283

0.305

0.326

0.349

0.371

0.393

0.414

0.432

0.446

0.466

0.486

0.507

0.527

0.547

0.568

0.588

0.608

0.629

0.649

0.670

0.690

0.711

0.733

0.754

0.776

0.797

0.819

0.841

0.864

0.886

0.909

0.931

0.954

0.977
1.4

0.000

0.014

0.029

0.044

0.060

0.075

0.092

0.108

0.125

0.142

0.159

0.177

0.195

0.214

0.233

0.261

0.282

0.303

0.324

0.346

0.368

0.389

0.410

0.429

0.444

0.464

0.484

0.505

0.526

0.546

0.567

0.587

0.608

0.628

0.649

0.669

0.690

0.711

0.732

0.754

0.775

0.797

0.819

0.841

0.864

0.886

0.908

0.931

0.954

0.977 
Table 4.5.2-1b $\quad\left(p_{1}=2 \mathrm{~atm}\right)$

Inlet Void

Fraction

\begin{tabular}{|c|c|}
\hline$\alpha_{1}$ & 0.2 \\
\hline 0.00 & 22.589 \\
\hline 0.02 & 0.691 \\
\hline 0.04 & 0.656 \\
\hline 0.06 & 0.614 \\
\hline 0.08 & 0.646 \\
\hline 0.10 & 0.868 \\
\hline 0.12 & 1.147 \\
\hline 0.14 & 0.919 \\
\hline 0.16 & 0.456 \\
\hline 0.18 & 0.385 \\
\hline 0.20 & 0.343 \\
\hline 0.22 & 0.311 \\
\hline 0.24 & 0.283 \\
\hline 0.26 & 0.261 \\
\hline 0.28 & 0.238 \\
\hline 0.30 & 0.218 \\
\hline 0.32 & 0.202 \\
\hline 0.34 & 0.187 \\
\hline 0.36 & 0.173 \\
\hline 0.38 & 0.161 \\
\hline 0.40 & 0.150 \\
\hline 0.42 & 0.139 \\
\hline 0.44 & 0.129 \\
\hline 0.46 & 0.120 \\
\hline $\begin{array}{l}0.48 \\
0.50\end{array}$ & $\begin{array}{l}0.112 \\
0.104\end{array}$ \\
\hline $\begin{array}{l}0.50 \\
0.52\end{array}$ & 0.097 \\
\hline 0.54 & 0.091 \\
\hline 0.56 & 0.085 \\
\hline 0.58 & 0.079 \\
\hline 0.60 & 0.074 \\
\hline 0.62 & 0.070 \\
\hline $\begin{array}{l}0.64 \\
0.66\end{array}$ & $\begin{array}{l}0.065 \\
0.061\end{array}$ \\
\hline 0.68 & 0.058 \\
\hline 0.70 & 0.054 \\
\hline 0.72 & 0.051 \\
\hline 0.74 & 0.049 \\
\hline 0.76 & 0.046 \\
\hline 0.78 & 0.044 \\
\hline 0.80 & 0.041 \\
\hline 0.82 & 0.039 \\
\hline 0.84 & 0.038 \\
\hline 0.86 & 0.036 \\
\hline 0.88 & 0.034 \\
\hline 0.90 & 0.033 \\
\hline 0.92 & 0.031 \\
\hline 0.94 & 0.030 \\
\hline 0.96 & 0.028 \\
\hline 0.98 & 0.027 \\
\hline
\end{tabular}

Slatic Pressure Increase, $\left(p_{2}-p_{1}\right)$ (psi)

Homologous Parameter

$\begin{array}{lllllll}0.4 & 0.6 & 0.8 & 1.0 & 1.2 & 1.4\end{array}$

22.357

21.787

22.131

21.911

21.515

21.679

21.229

20.678

20.778

21.115

20.712

20.145

20.327

20.304

19.624

19.876

19.892

19.112

19.424

19.475

18.607

18.108

17.615

18.972

19.056

18.518

18.631

18.064

18.203

5.469

2.346

1.849

1.606

1.407

17.608

17.772

17.140

16.428

17.338

16.875

5.595

16.268

1.238

1.091

4.793

9.377

4.124

8.283

0.964

3.553

7.270

0.854

3.064

6.337

2.642

5.492

0.757

2.279

4.737

0.670

1.966

0.594

1.695

0.526

1.462

4.070

3.490

2.979

0.465

1.259

2.537

1.084

2. 154

0.363

0.321

0.932

1.825

0.800

1.542

0.685

1.299

0.250

0.585

1.090

0.499

0.912

0.760

0.630

0.519

0.425

0.346

0.279

0.224

0.178

0.141

0.110

0.086

0.067

0.053

0.042

0.034

0.029

0.027

0.026

0.026

0.026
21.693

21.334

20.969

20.598

20.220

19.836

19.446

19.051

18.650

18.244

17.831

17.414

16.986

16.509

15.837

10.960

9.983

9.013

8.061

7.240

6.264

5.447

4.701

4.036

3.438

2.909

2.449

2.050

1.707

1.414

1.164

0.952

0.773

0.621

0.495

0.390

0.302

0.231

0.174

0.128

0.093

0.065

0.045

0.031

0.023

0.018

0.017

0.018

0.021

0.024
21.478

21.152

20.819

20.479

20.130

19.775

19.413

19.042

18.663

18.273

17.878

17.476

17.066

16.623

16.096

12.241

11.373

10.500

9.617

8.728

7.843

6.976

6.140

5.350

4.627

3.956

3.350

2.811

2.341

1.933

1.582

2.284

1.031

0.819

0.641

0.493

0.372

0.275

0.197

0.135

0.087

0.053

0.027

0.012

0.002

$-0.001$

0.000

0.005

0.012

0.020
21.264

20.967

20.660

20.346

20.024

19.692

19.351

19.002

18.645

18.280

17.905

17.523

17.133

16.718

16.250

12.938

12.120

11.319

10.504

9.673

8.829

7.983

7.143

6.323

5.551

4.808

4.111

3.475

2. 905

2. 401

1.963

1.585

1. 263

0.991

0.765

0.577

0.423

0.300

0.201

0.125

0.067

0.025

$-0.003$

$-0.020$

$-0.029$

$-0.029$

$-0.0: \div$

$-0.0:$

0.0

$0.0:$ 
Table 4.5.2-1b
(coatlaued) $\quad\left(p_{1}=2 \mathrm{~atm}\right)$

$v_{12}$ Liquid Flow Velociry (fect/sec)

Inlet Void

Fraction

$\begin{array}{ccc}\alpha_{1} & 0.2 & 0.4 \\ 0.00 & 2.251 & 4.503 \\ 0.02 & 2.253 & 4.477 \\ 0.04 & 2.252 & 4.454 \\ 0.06 & 2.246 & 4.432 \\ 0.08 & 2.237 & 4.411 \\ 0.10 & 2.223 & 4.390 \\ 0.12 & 2.211 & 4.371 \\ 0.14 & 2.201 & 4.352 \\ 0.16 & 2.198 & 4.335 \\ 0.18 & 2.199 & 4.320 \\ 0.20 & 2.188 & 4.341 \\ 0.22 & 2.178 & 4.351 \\ 0.24 & 2.170 & 4.347 \\ 0.26 & 2.170 & 4.337 \\ 0.28 & 2.161 & 4.326 \\ 0.30 & 2.148 & 4.315 \\ 0.32 & 2.149 & 4.304 \\ 0.34 & 2.145 & 4.292 \\ 0.36 & 2.139 & 4.281 \\ 0.38 & 2.132 & 4.269 \\ 0.40 & 2.126 & 4.257 \\ 0.42 & 2.120 & 4.244 \\ 0.44 & 2.114 & 4.232 \\ 0.46 & 2.107 & 4.220 \\ 0.48 & 2.101 & 4.207 \\ 0.50 & 2.094 & 4.195 \\ 0.52 & 2.088 & 4.183 \\ 0.54 & 2.082 & 4.170 \\ 0.56 & 2.076 & 4.158 \\ 0.58 & 2.070 & 4.146 \\ 0.60 & 2.064 & 4.134 \\ 0.62 & 2.058 & 4.122 \\ 0.64 & 2.053 & 4.110 \\ 0.66 & 2.047 & 4.098 \\ 0.68 & 2.042 & 4.086 \\ 0.70 & 2.036 & 4.075 \\ 0.72 & 2.031 & 4.063 \\ 0.74 & 2.025 & 4.051 \\ 0.76 & 2.019 & 4.040 \\ 0.78 & 2.014 & 4.028 \\ 0.80 & 2.008 & 4.016 \\ 0.82 & 2.003 & 4.005 \\ 0.84 & 1.997 & 3.993 \\ 0.86 & 1.991 & 3.981 \\ 0.88 & 1.986 & 3.970 \\ 0.90 & 1.980 & 3.959 \\ 0.92 & 1.975 & 3.947 \\ 0.94 & 1.969 & 3.936 \\ 0.96 & 1.964 & 3.925 \\ 0.98 & 1.959 & 3.915 \\ & & \end{array}$

Homologous Parameter

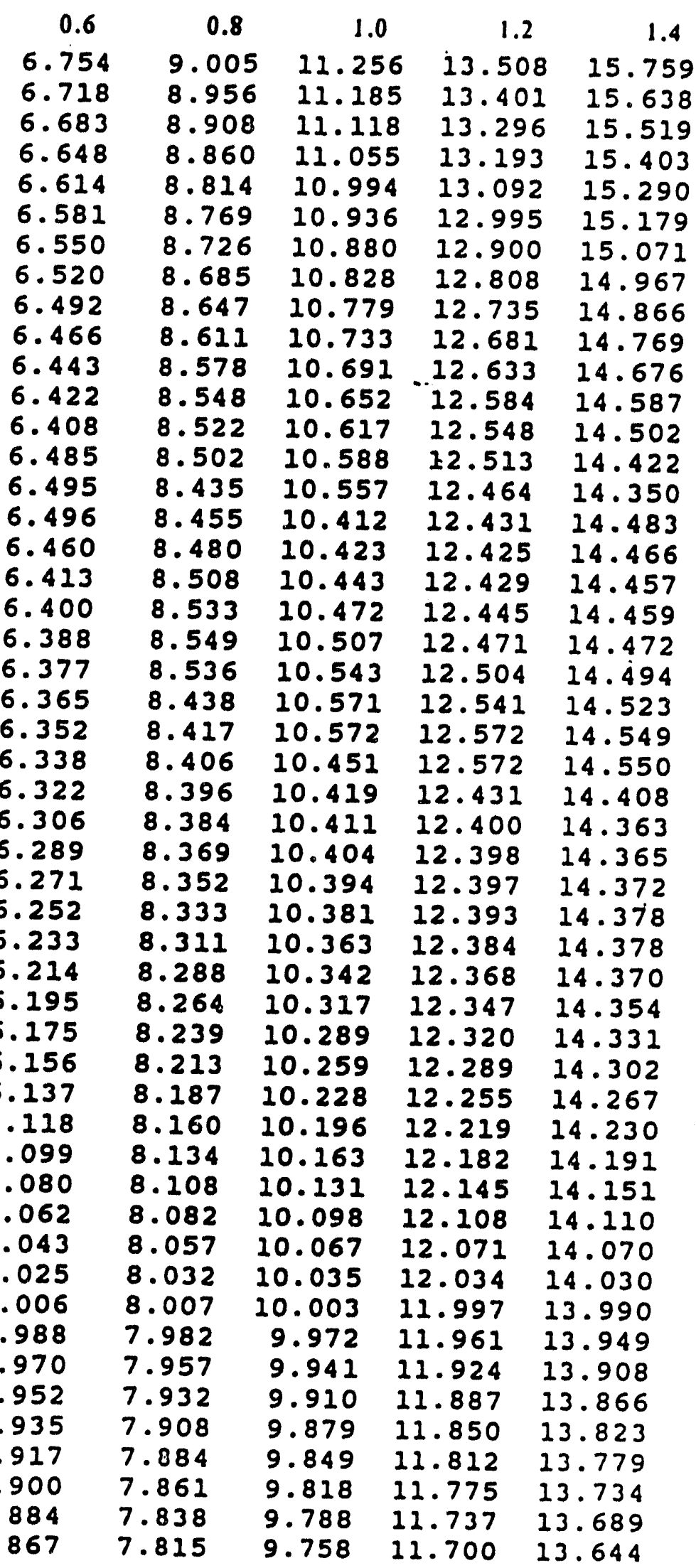


Table 4.5.2-1b $\quad\left(p_{1}=2 \mathrm{~atm}\right)$ (contlaued)

$v_{c^{2}}$ Gas Flow Velocity (fecl/sec)

Inlet Void

Fraction



Homologous Parameter

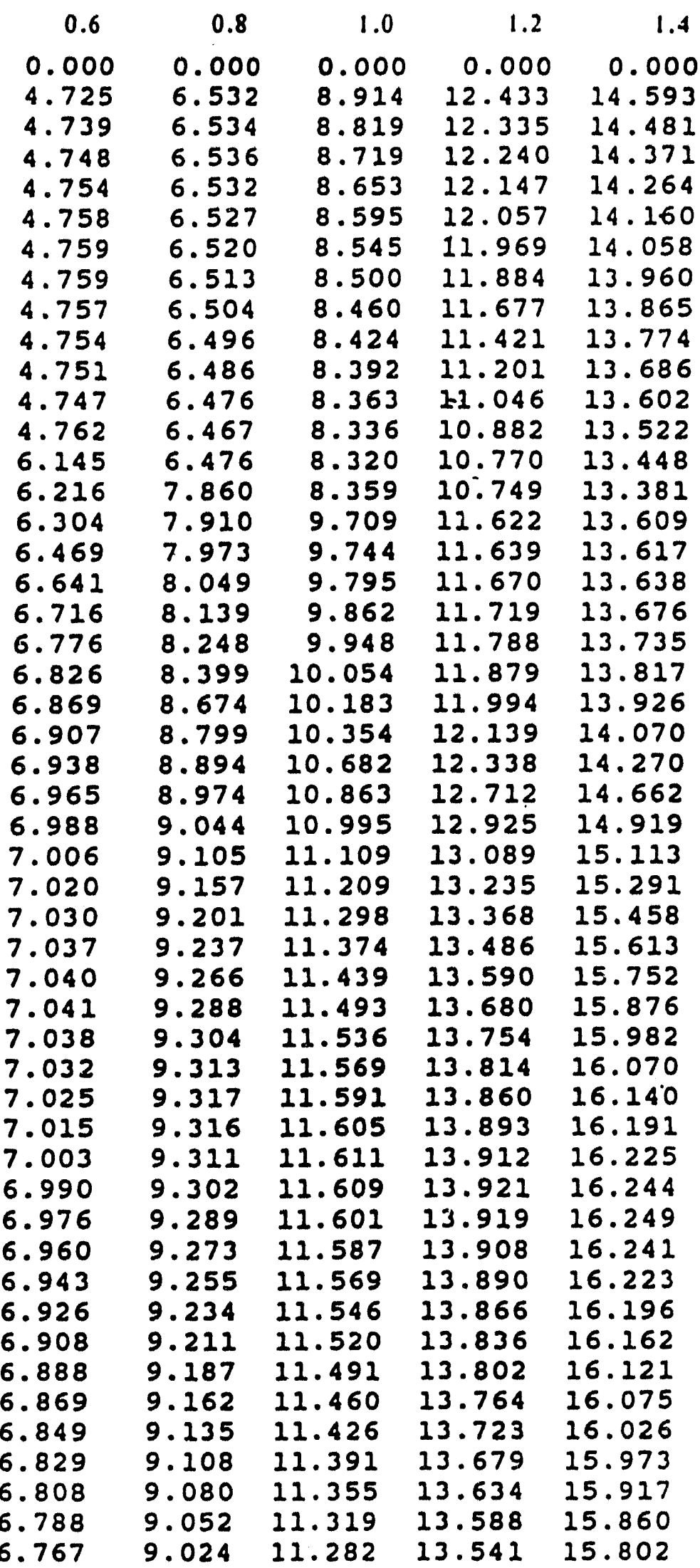


Table 4.5.2-1b $\quad\left(p_{1}=2 \mathrm{~atm}\right)$

(continued)

$\alpha_{2}$ Void Fraction

Inlet Void

Fraction

\begin{tabular}{|c|c|}
\hline$\alpha_{1}$ & 0.2 \\
\hline 0.00 & 0.000 \\
\hline 0.02 & 0.022 \\
\hline $\begin{array}{l}0.04 \\
0.06\end{array}$ & 0.041 \\
\hline $\begin{array}{l}0.06 \\
0.08\end{array}$ & 0.059 \\
\hline $\begin{array}{l}0.08 \\
0.10\end{array}$ & 0.074 \\
\hline $\begin{array}{l}0.10 \\
0.12\end{array}$ & 0.088 \\
\hline $\begin{array}{l}0.12 \\
0.14\end{array}$ & 0.101 \\
\hline $\begin{array}{l}0.14 \\
0.16\end{array}$ & 0.119 \\
\hline $\begin{array}{l}0.16 \\
0.18\end{array}$ & 0.139 \\
\hline $\begin{array}{l}0.18 \\
0.20\end{array}$ & 0.158 \\
\hline $\begin{array}{l}0.20 \\
0.22\end{array}$ & .0 .175 \\
\hline 0.22 & 0.193 \\
\hline 0.24 & 0.211 \\
\hline 0.26 & 0.229 \\
\hline 0.28 & 0.248 \\
\hline 0.30 & 0.266 \\
\hline 0.32 & 0.286 \\
\hline 0.34 & 0.305 \\
\hline 0.36 & 0.324 \\
\hline 0.38 & .0 .344 \\
\hline 0.40 & 0.363 \\
\hline 0.42 & 0.382 \\
\hline 0.44 & 0.402 \\
\hline 0.46 & 0.422 \\
\hline 0.48 & 0.441 \\
\hline $\begin{array}{l}0.50 \\
0.52\end{array}$ & $\begin{array}{l}0.461 \\
0.481\end{array}$ \\
\hline $\begin{array}{l}0.52 \\
0.54\end{array}$ & $\begin{array}{l}0.481 \\
0.501\end{array}$ \\
\hline 0.56 & 0.522 \\
\hline 0.58 & 0.542 \\
\hline 0.60 & 0.563 \\
\hline 0.62 & 0.583 \\
\hline $\begin{array}{l}0.64 \\
0.66\end{array}$ & $\begin{array}{l}0.604 \\
0.625\end{array}$ \\
\hline 0.68 & 0.646 \\
\hline 0.70 & 0.668 \\
\hline 0.72 & 0.689 \\
\hline 0.74 & 0.710 \\
\hline 0.76 & 0.732 \\
\hline 0.78 & 0.754 \\
\hline 0.80 & 0.775 \\
\hline 0.82 & 0.797 \\
\hline 0.84 & 0.819 \\
\hline 0.86 & 0.842 \\
\hline 0.88 & 0.864 \\
\hline 0.90 & 0.886 \\
\hline 0.92 & 0.909 \\
\hline 0.94 & 0.931 \\
\hline 0.96 & 0.954 \\
\hline 0.98 & 0.977 \\
\hline
\end{tabular}

Homologous Parameter

0.4
0.000
0.015
0.031
0.046
0.061
0.077
0.093
0.110
0.127
0.145
0.170
0.192
0.212
0.231
0.250
0.269
0.288
0.307

0.6

0.000

0.015

0.030

0.045

0.060

0.076

0.092

0.109

0.126

0.143

0.161

0.180

0.199

0.230

0.252

0.273

0.290

0.305

0.307

0.327

0.346

0.365

0.384

0.404

0.424

0.443

0.463

0.483

0.503

0.523

0.544

0.564

0.585

0.605

0.626

0.647

0.668

0.690

0.711

0.732

0.754

0.776

0.798

0.820

0.842

0.864

0.886

0.909

0.931

0.954

0.977
0.325

0.345

0.365

0.385

0.405

0.425

0.445

0.465

0.485

0.505

0.525

0.545

0.565

0.586

0.606

0.627

0.648

0.669

0.690

0.711

0.733

0.754

0.776

0.798

0.820

0.842

0.864

0.886

0.909

0.931

0.954

0.977

$\begin{array}{cc}0.8 & \\ 0.000 & 0.00 \\ 0.015 & 0 \\ 0.029 & 0 . \\ 0.045 & 0 \\ 0.060 & 0 \\ 0.076 & 0 \\ 0.092 & 0 \\ 0.0108 & 0\end{array}$

1.0

0.000

0.014

0.028

0.043

0.058

0.074

0.090

0.106

0.125

0.142

0.160

0.178

0.197

0.216

0.231

0.254

0.278

0.301

0.325

0.347

0.367

0.381

0.401

0.422

0.442

0.463

0.484

0.504

0.524

0.545

0.565

0.586

0.606

0.627

0.648

0.669

0.690

0.711

0.733

0.754

0.776

0.798

0.819

0.842

0.864

0.886

0.909

0.931

0.954

0.977

0.123

0.140

0.158

0.176

0.194

0.213

0.232

0.243

0.265

0.288

0.312

0.336

0.359

0.382

0.404

0.418

0.438

0.459

0.481

0.502

0.523

0.544

0.565

0.585

0.606

0.627

0.648

0.669

0.690

0.711

0.732

0.754

0.776

0.797

0.819

0.841

0.864

0.886

0.909

0.932

0.954

0.977

1.2
0.000
0.012
0.025
0.038
0.051
0.064
0.078
0.093
0.109
0.126
0.145
0.163
0.182
0.201
0.220
0.239
0.261
0.283
0.305
0.328
0.352
0.375
0.398
0.420
0.435
0.455
0.477
0.499
0.520
0.542
0.35

1.4

0.000

0.012

0.025

0.038

0.052

0.066

0.080

0.094

0.110

0.125

0.141

0.157

0.174

0.191

0.209

0.238

0.259

0.280

0.302

0.325

0.348

0.371

0.393

0.415

0.431

0.451

0.473

0.496

0.518

0.542

0.540

0.563

0.561

0.584

0.583

0.605

0.604

0.625

0.626

0.646

0.668

0.668

0.689

0.689

0.711

0.710

0.732

0.732

0.754

0.754

0.775

0.775

0.797

0.797

0.819

0.819

0.841

0.864

0.864

0.886

0.886

0.909

0.909

0.931

0.931

0.954

0.954

0.977

0.977 
Table 4.5.2-1c $\quad\left(p_{1}=1 \mathrm{~atm}\right)$

Inlet Void

Fraction

$\alpha_{1}$

0.00

0.02

0.04

0.06

0.08

0.10

0.12

0.14

0.16

0.18

0.20

0.22

0.24

0.26

0.28

0.30

0.32

0.34

0.36

0.38

0.40

0.42

0.44

0.46

0.48

0.50

0.52

0.54

0.56

0.58

0.60

0.62

0.64

0.66

0.68

0.70

0.72

0.74

0.76

0.78

0.80

0.82

0.84

0.86

0.88

0.90

0.92

0.94

0.96

0.98
0.2

22.589

0.675

0.643

0.601

0.649

0.928

1.285

1.012

0.444

0.372

0.330

0.297

0.270

0.248

0.225

0.205

0.189

0.174

0.160

0.148

0.137

0.126

0.116

0.107

0.099

0.091

0.084

0.078

0.072

0.066

0.061

0.057

0.052

0.048

0.045

0.042

0.038

0.036

0.033

0.031

0.029

0.027

0.025

0.023

0.021

0.020

0.018

0.017

0.016

0.014
0.4

22.357

21.902

21.448

20.998

20.550

20.105

19.662

19.218

18.776

18.332

17.883

2.585

1.854

1.604

1.403

1.231

1.084

0.955

0.844

0.746

0.659

0.582

0.514

0.453

0.399

0.352

0.309

0.271

0.237

0.207

0.181

0.157

0.137

0.118

0.103

0.089

0.077

0.066

0.058

0.050

0.043

0.037

0.032

0.028

0.025

0.022

0.021

0.018

0.017

0.015

Static Pressure Increase, $\left(p_{2}-p_{1}\right)(p s i)$

Homologous Parameter

$\begin{array}{llllll}0.6 & 0.8 & 1.0 & 1.2 & 1.4\end{array}$

$\begin{array}{llll}22.131 & 21.911 & 21.693 & 21.478\end{array}$

$\begin{array}{llll}21.789 & 21.622 & 21.445 & 21.269\end{array}$

$\begin{array}{llll}21.441 & 21.325 & 21.188 & 21.048\end{array}$

$\begin{array}{llll}21.088 & 21.019 & 20.919 & 20.815\end{array}$

$\begin{array}{llll}20.727 & 20.706 & 20.642 & 20.572\end{array}$

20.360

19.987

19.608

20.383

20.353

20.052

20.054

19.711

19.745

20.317

20.051

19.773

19.425

19.484

18.828

18.429

19.362

19.094

28.636

18.022

17.572

18.259

18.752

18.401

19.182

.28 .869

18.542

18.199

17.435

10.393

5.016

4.286

3.669

3.146

2.700

2. 319

1.993

1. 711

1.471

1.262

1.083

0.927

0.793

0.677

0.575

0.488

0.412

0.348

0.291

0.242

0.202

0.166

0.136

0.111

0.090

9.243

7.975

6.896

5.925

5.062

4.309

3.661

3.102

2. 622

2.212

1.863

1.566

1.313

1.096

0.912

0.756

0.622

0.510

0.415

0.334

0.267

0.212

0.165

0.127

0.073

0.096

0.059

0.072

0.048

0.053

0.039

0.038

0.028

0.026

0.020

0.016

0.022

0.019

0.013

0.017

0.012

0.015

0.012

0.014

0.013

18.036

17.645

17.201

12.285

11.229

10.149

9.062

7.989

6.958

5.995

5.119

4.341

3.665

3.072

2.562

2.128

1.759

1.446

1.182

0.960

0.774

0.618

0.487

0.380

0.291

0.219 
able 4.5.2-1c $\quad\left(p_{1}=1 \mathrm{~atm}\right)$

(continued)

$v_{12}$ Liquid Flow Velocily (fect/sec)

Inlet Void

Fraction

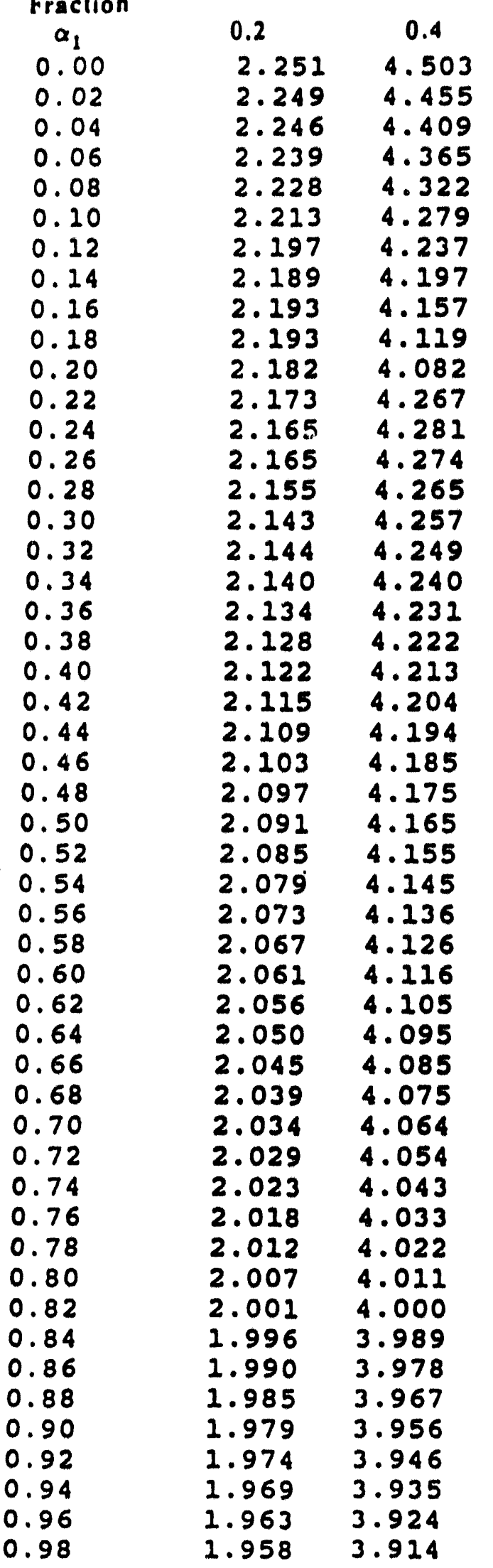

Homologous Parameler

$\begin{array}{lllll}0.6 & 0.8 & 1.0 & 1.2 & 1.4\end{array}$

$\begin{array}{lll}6.754 & 9.005 & 11.256\end{array}$

$13.508 \quad 15.759$

6.685

$8.912 \quad 11.125$

13.351

15.580

6.617

8.820

11.000

13.194

15.402

6.549

8.730

10.880

13.040

15.225

6.483

8.641

10.765

12.887

15.051

6.418

8.554

10.652

6.354

8.468

6.291

6.230

8.385

10.545

12.736

14.878

12.587

14.708

8.303

10.442

6.171

6.114

8.224

10.342

12.441

12.296

14.540

14.374

8.146

10.246

10.152

6.058

6.006

8.072

10.061

12.155

14.212

12.016

14.053

8.000

9.972

11.881

13.897

6.223

7.933

9.889

11.782

13.745

6.242

7.946

9.812

11.693

23.598

6.255

7.955

9.731

9.712

6.251

7.979

6.205

8.014

9.712

6.202

8.057

6.204

8.100

6.206

9.732

9.772

8.132

6.207

8.083

9.829

11.606

13.456

6.207

8.072

9.895

9.956

11.605

11.553

11.517

21.502

11.509

11.540

11.595

11.666

9.971

11.744

11.790

9.906

9.940

11.707

11.755

11.818

9.980

10.017

6.183

6.174

6.164

6.153

6.142

6.129

6.116

6.103

6.089

6.075

6.060

6.045

6.030

6.014

5.998

5.981

5.965

5.948

5.932

5.915

5.898

5.882

8.145

8.151

10.048

11.880

11.936

8.152

10.074

10.092

11.982

13.323

13.447

13.385

13.343

13.322

13.326

13.355

13.410

13.482

13.552

13.495

13.504

13.585

13.674

8.145

10.203

12.018

13.760

8.137

10.109

12.044

13.837

8.127

10.108

12.061

13.902

8.115

8.101

10.104

10.095

12.070

12.072

12.068

8.086

10.083

10.069

12.058

8.052

10.051

8.033

10.032

12.045

12.027

12.006

10.012

7.014

9.987

9.962

11.982

11.955

11.925

7.950

9.936

9.908

9.879

9.850

9.819

9.789

11.892

11.857

11.820

11.781

11.742

11.702

13.954

13.992

14.019

14.035

14.043

14.043

14.036

14.024

14.005

13.982

13.954

13.921

13.883

13.842

13.797

7.859

9.758

$13.7: 9$

5.866

7.814

13.044 
Table 4.5.2-1c $\quad\left(p_{1}-1\right.$ atc

(contlaued)

$$
\checkmark \text { Gas Flow Velocily (feet/sec) }
$$

Inlet Vold

Fraction

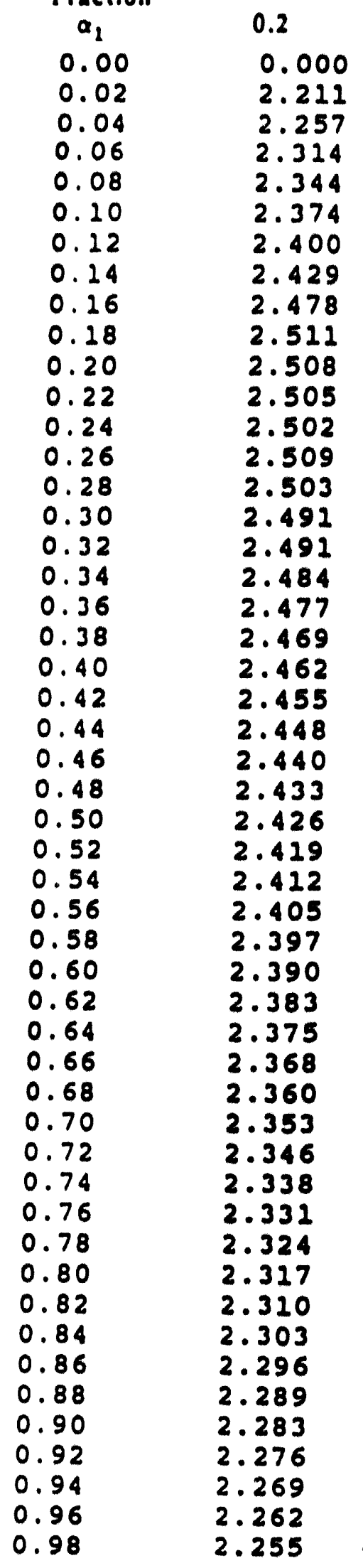

Homologous Parameter

\begin{tabular}{|c|c|c|c|c|c|}
\hline 0.4 & 0.6 & 0.8 & 1.0 & 1.2 & 1.8 \\
\hline $\begin{array}{l}0.000 \\
3.127 \\
3.089 \\
3.064 \\
3.042 \\
3.022 \\
3.003 \\
.984 \\
.966 \\
.947 \\
.929 \\
.562 \\
.630 \\
.654 \\
.673 \\
.691 \\
.705\end{array}$ & 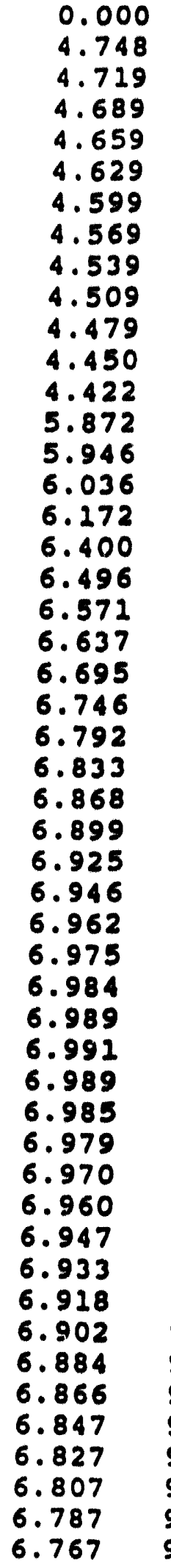 & $\begin{array}{l}0.000 \\
6.669 \\
6.594 \\
6.518 \\
6.455 \\
6.395 \\
6.339 \\
6.285 \\
6.229 \\
6.179 \\
6.131 \\
6.084 \\
6.038 \\
5.996 \\
7.373 \\
7.408 \\
7.464 \\
7.541 \\
7.639 \\
7.759 \\
7.912 \\
8.203 \\
8.401 \\
8.534 \\
8.649 \\
8.752 \\
8.844 \\
8.926 \\
8.998 \\
9.060 \\
9.113 \\
9.157 \\
9.193 \\
9.220 \\
9.241 \\
9.254 \\
9.261 \\
9.262 \\
9.108 \\
9.051 \\
9.023 \\
9.259 \\
9.250 \\
9.238 \\
9.203 \\
9.183 \\
9.02 \\
9\end{array}$ & 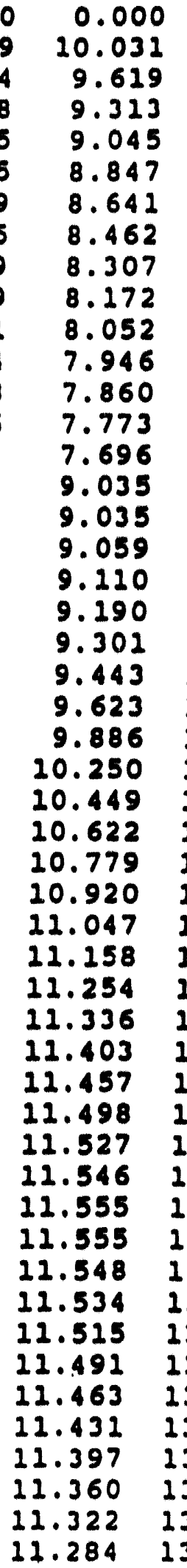 & 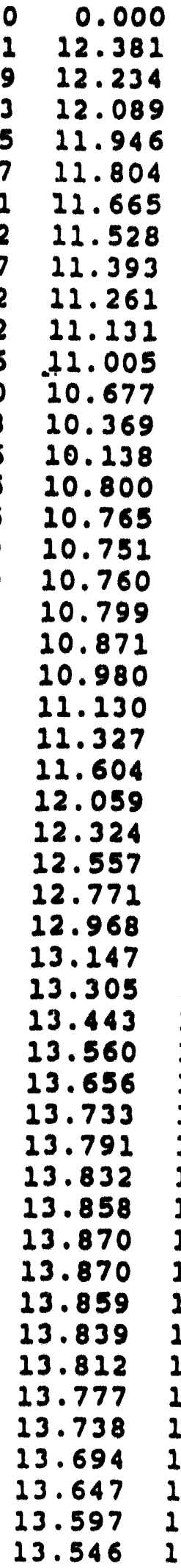 & $\begin{array}{l}13.229 \\
13.078 \\
12.931 \\
12.787 \\
12.648 \\
12.04 \\
12.514 \\
12.389 \\
12.580 \\
12.540 \\
12.522 \\
12.532 \\
12 \\
12.574 \\
12.655 \\
12.780 \\
12.957 \\
12 . \\
13.206 \\
13.668 \\
14.04\end{array}$ \\
\hline
\end{tabular}


Table 4.5.2-1c $\quad\left(p_{1}=1 \mathrm{~atm}\right)$ (continued)

$a_{2}$ Void Fraction

Inlet Void

Fraction



Homologous Parameter

$\begin{array}{lll}0.4 & 0.6 & 0.8\end{array}$

0.000

0.000

0.000

0.010

0.010

0.010

0.020

0.020

0.020

0.031

0.030

0.042

0.041

0.053

0.042

0.053

0.065

0.077

0.090

0.065

0.077

0.052

0.064

0.076

0.089

0.089

0.102

0.102

0.217

0.126

0.130

0.145

0.126

0.176

0.200

0.220

0.198

0.130

0.144

0.260

0.239

0.259

0.279

0.299

0.319

0.338

0.358

0.378

0.399

0.222

0.245

0.266

0.183

0.207

0.232

0.258

0.282

0.304

0.325

0.347

0.285

0.321

0.336

0.369

0.391

0.412

0.439

0.459

0.434

0.455

0.480

0.476

0.500

0.498

0.521

0.541

0.519

0.540

0.561

0.562

0.582

0.603

0.625

0.354

0.375

0.399

0.422

0.446

0.469

0.491

0.514

0.536

0.558

0.580

0.602

0.623

0.604

0.646

0.646

0.668

0.667

0.645

0.667

0.688

0.720

0.689

0.710

0.710

0.732

0.732

0.732

0.753

0.775

0.775

0.797

0.819

0.842

0.864

0.886

0.909

0.931

0.954

0.977
0.754

0.775

0.797

0.819

0.841

0.864

0.886

0.909

0.931

0.954

0.977
0.797

0.819

0.841

0.864

0.886

0.909

0.931

0.954

0.977
1.0

0.000

0.008

0.018

0.027

0.038

0.049

0.061

0.073

0.086

0.099

0.123

0.127

0.142

0.258

0.274

0.190

0.211

0.235

0.259

0.286

0.313

0.340

0.367

0.390

0.409

0.434

0.459

0.483

0.507

0.531

0.554

0.577

0.599

0.621

0.643

0.665

0.687

0.709

0.731

0.753

0.775

0.797

0.829

0.842

0.864

0.886

0.909

0.931

0.954

0.977

\section{2}

0.000

0.008

0.017

0.026

0.036

0.045

0.056

0.066

0.077

0.089

0.101

0.123

0.129

0.245

0.162

0.185

0.205

0.226

0.248

0.272

0.298

0.324

0.351

0.379

0.404

0.423

0.448

0.474

0.500

0.525

0.549

0.573

0.596

0.619

0.642

0.664

0.687

0.709

0.731

0.753

0.775

0.797

0.819

0.841

0.864

0.886

0.909

0.931

0.954

0.977

1.4

0.000

0.009

0.018

0.027

0.037

0.047

0.057

0.068

0.079

0.091

0.203

0.115

0.129

0.142

0.157

0.172

0.203

0.223

0.244

0.266

0.290

0.315

0.342

0.369

0.395

0.416

0.440

0.466

0.493

0.519

0.544 
Table 4.5.2-1d $\quad\left(p_{1}=0.5 \mathrm{~atm}\right)$

Stalle Pressure Increase, $\left(p_{2}-p_{1}\right)(p s i)$

Inlet Vold

Fraction

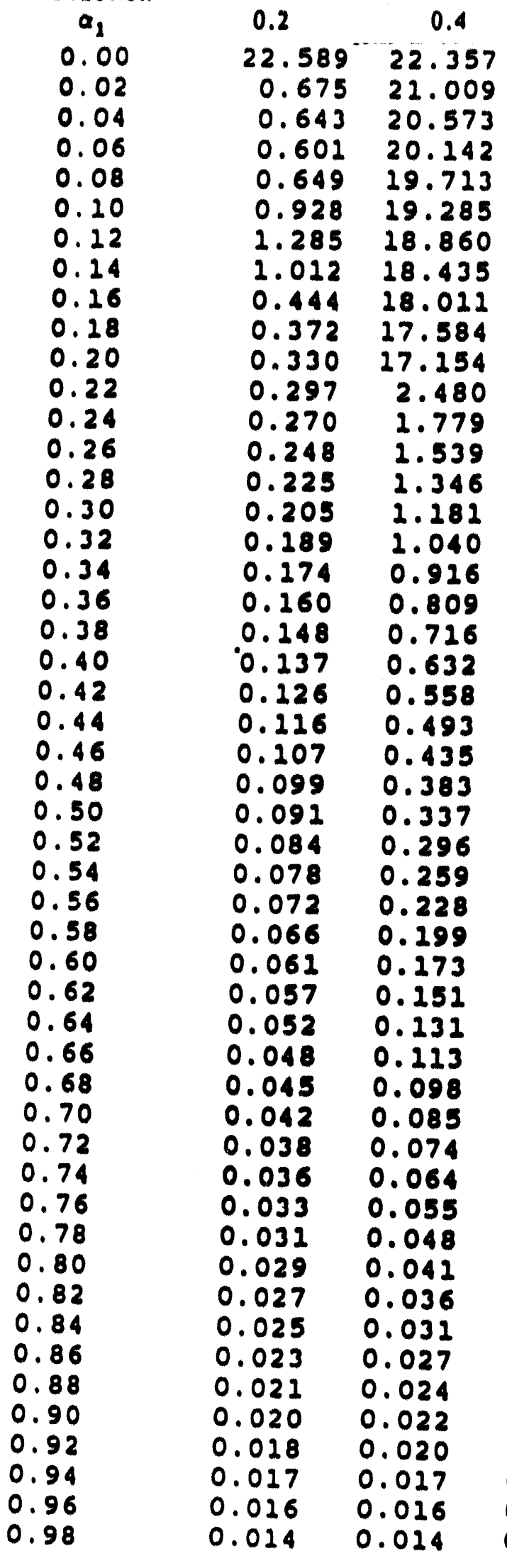

\section{Homologous Parameter}

$\begin{array}{ccccc}0.6 & 0.8 & 1.0 & 1.2 & 1.4 \\ 22.131 & 21.911 & 21.693 & 21.478 & 21.264 \\ 21.789 & 21.622 & 21.445 & 21.269 & 21.091 \\ 21.441 & 21.325 & 21.188 & 21.048 & 20.905 \\ 21.088 & 21.019 & 20.919 & 20.815 & 20.708 \\ 20.727 & 20.706 & 20.642 & 20.572 & 20.498 \\ 20.360 & 20.383 & 20.353 & 20.317 & 20.276 \\ 19.987 & 20.052 & 20.054 & 20.051 & 20.041 \\ 19.608 & 19.711 & 19.745 & 19.773 & 19.793 \\ 19.221 & 19.362 & 19.425 & 19.484 & 19.530 \\ 18.828 & 19.003 & 19.094 & 19.182 & 19.257 \\ 18.429 & 18.636 & 18.752 & 18.869 & 18.968 \\ 18.022 & 18.259 & 18.401 & 18.542 & 18.666 \\ 17.572 & 17.869 & 18.036 & 18.199 & 18.350 \\ 5.908 & 17.435 & 17.645 & 17.833 & 18.013 \\ 5.016 & 10.393 & 17.201 & 17.431 & 17.644 \\ 4.286 & 9.143 & 12.285 & 13.746 & 17.208 \\ 3.669 & 7.975 & 12.229 & 12.854 & 13.766 \\ 3.146 & 6.896 & 10.149 & 12.936 & 12.952 \\ 2.700 & 5.925 & 9.062 & 10.977 & 12.098 \\ 2.319 & 5.062 & 7.989 & 9.982 & 12.199 \\ 1.993 & 4.309 & 6.958 & 8.965 & 10.258 \\ 1.711 & 3.661 & 5.995 & 7.944 & 9.285 \\ 1.471 & 3.102 & 5.119 & 6.945 & 8.293 \\ 1.262 & 2.622 & 1.341 & 5.994 & 7.304 \\ 1.083 & 2.212 & 3.665 & 5.117 & 6.342 \\ 0.927 & 1.863 & 3.072 & 4.334 & 5.445 \\ 0.793 & 1.566 & 2.562 & 3.628 & 4.609 \\ 0.677 & 1.313 & 2.128 & 3.011 & 3.847 \\ 0.575 & 1.096 & 1.759 & 2.480 & 3.175 \\ 0.488 & 0.912 & 1.446 & 2.028 & 2.592 \\ 0.412 & 0.756 & 1.182 & 1.645 & 2.093 \\ 0.348 & 0.622 & 0.960 & 1.322 & 1.671 \\ 0.291 & 0.510 & 0.774 & 1.052 & .318 \\ 0.242 & 0.415 & 0.618 & 0.828 & 1.024 \\ 0.202 & 0.334 & 0.487 & 0.642 & 0.780 \\ 0.166 & 0.267 & 0.380 & 0.489 & 0.581 \\ 0.136 & 0.212 & 0.291 & 0.364 & 0.420 \\ 0.121 & 0.165 & 0.219 & 0.263 & 0.291 \\ 0.090 & 0.127 & 0.160 & 0.183 & 0.189 \\ 0.073 & 0.096 & 0.114 & 0.121 & 0.110 \\ 0.059 & 0.072 & 0.078 & 0.073 & 0.052 \\ 0.048 & 0.053 & 0.051 & 0.037 & 0.009 \\ 0.039 & 0.038 & 0.031 & 0.012 & -0.020 \\ 0.031 & 0.028 & 0.017 & -0.004 & -0.038 \\ 0.026 & 0.020 & 0.008 & -0.013 & -0.045 \\ 0.022 & 0.016 & 0.003 & -0.017 & -0.045 \\ .019 & 0.013 & 0.002 & -0.015 & -0.039 \\ .017 & 0.012 & 0.003 & -0.011 & -0.029 \\ .015 & 0.012 & 0.006 & -0.003 & -0.016 \\ 0.014 & 0.013 & 0.010 & 0.005 & -0.001\end{array}$


Table 4.5.2-1d $\quad\left(p_{1}=0.5 \mathrm{~atm}\right)$

(contlaued)

$v_{12}$ Liquid Flow Velocily (recl/sec)

Inlel Vold

Fraction



0.00

0.02

0.04

0.06

0.08

0.10

0.12

0.14

0.16

0.18

0.20

0.22

0.24

0.26

0.28

0.30

0.32

0.34

0.36

0.38

0.40

0.42

0.44

0.46

0.48

0.50

0.52

0.54

0.56

0.58

0.60

0.62

0.64

0.66

0.68

0.70

0.72

0.74

0.76

0.78

0.80

0.82

0.84

0.86

0.88

0.90

0.92

0.94

0.96

0.98
0.2

2.251

2.231

2.214

2.200

2.188

2.177

2. 268

2.146

2.182

2.183

2.173

2.163

2.255

2.155

2.246

2.233

2.135

2.130

2. 125

2. 119

2. 113

2. 107

2.101

2.096

2.090

2.084

2.078

2.073

2.067

2.061

2.056

2.051

2.046

2.041

2.035

2.030

2.025

2.020

2.015

2.010

2.005

1.999

1.994

1.989

1.983

1.978

1.973

1.968

1.963

1.958

\section{Homologous Parameter}

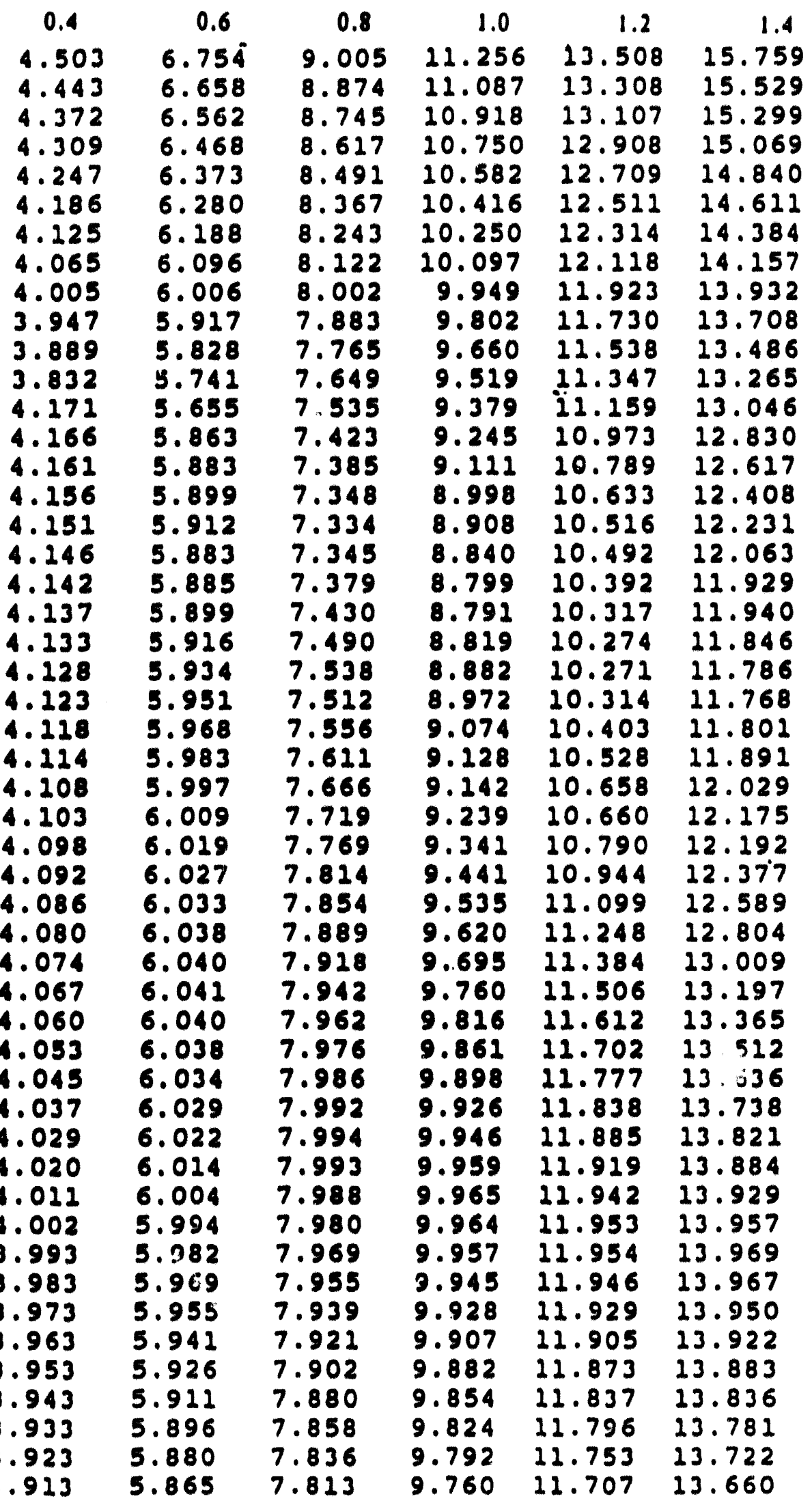


$\underset{\text { (contlaued) }}{\text { Table } 4.5 .2-1 d} \quad\left(p_{1}=0.5 \mathrm{~atm}\right)$

$v_{\text {ca }}$ Gas Flow Velocily (fect/sec)

Inlet Vold

Fraction

$$
a_{1}
$$

0.00

0.02

0.04

0.06

0.08

0.10

0.12

0.14

0.26

0.18

0.20

0.22

0.24

0.26

0.28

0.30

0.32

0.34

0.36

0.38

0.40

0.42

0.44

0.46

0.48

0.50

0.52

0.54

0.56

0.58

0.60

0.62

0.64

0.66

0.68

0.70

0.72

0.74

0.76

0.78

0.80

0.82

0.84

0.86

0.88

0.90

0.92

0.94

0.96

0.98
0.2

0.000

3.485

3.325

3.166

3.010

2.855

2.704

2.556

2.457

2. 497

2.495

2. 492

2.490

2.497

2.492

2.480

2. 481

2.474

2.467

2.460

2.453

2.446

2.439

2.432

2.425

2.419

2.412

2.405

2.398

2. 391

2.384

2.377

2.370

2.363

2.356

2.349

2.342

2.335

2.328

2.322

2.315

2.308

2.301

2. 295

2.288

2.281

2.275

2. 268

2. 262

2.255

Homologous Parameter

$\begin{array}{lll}0.4 & 0.6 & 0.8\end{array}$

0.000

3.267

3.187

3.100

3.036

2.985

2.940

2.897

2.856

2.817

2.780

2.744

4.510

4.536

4.559

4.579

4.598

4.614

4.629

4.642

4.654

4.664

4.673

4.680

4.686

4.690

4.692

4.694

4.694

4.692

4.690

4.687

4.682

4.677

4.671

4.664

4.656

4.647

4.638

4.628

4.618

4.607

4.596

4.584

4.573

4.560

4.548

4.535

4.523

4.510

0.6

0.000

4.847

4.768

4.693

4.627

4.559

4.493

4.429

4.365

4.305

4.246

4.289

4.133

5.493

5.563

5.643

5.754

6.000

6.134

6.229

6.314

6.391

6.463

6.529

6.591

6.647

6.697

6.743

6.783

6.818

6.847

6.872

6.892

6.908

6.919

6.926

6.930

6.930

6.927

6.921

6.913

6.903

6.890

6.876

6.860

6.843

6.825

6.806

6.786

6.766
0.000

7.147

6.955

6.780

6.599

6.456

6.323

6.289

6.076

5.962

5.862

5.767

5.672

5.583

6.813

6.797

6.809

6.854

6.931

7.041

7.285

7.382

7.733

7.924

8.086

8.236

8.374

8.502

8.619

8.726

8.820

8.904

8.976

9.037

9.088

9.129

9.160

9.183

9.197

9.205

9.205

9.200

9.190

9.175

9.156

9.134

9.110

9.083

9.054

9.025

1.0

0.000

10.230

10.072

9.915

9.759

9.604

9.429

9.043

8.652

8.350

8.047

7.812

7.602

7.397

7.231

8.309

8.237

8.288

8.270

8.190

8.255

8.369

8.535

8.756

9.100 
Table 4.5.2-1d $\quad\left(p_{1}=0.5 \mathrm{~atm}\right)$

(continued)

$a_{2}$ Void Fraction

Inlel Void

Fraction

$$
a_{1}
$$

0.00

0.02

0.04

0.06

0.08

0.10

0.12

0.24

0.16

0.28

0.20

0.22

0.24

0.26

0.28

0.30

0.32

0.34

0.36

0.38

0.40

0.42

0.44

0.46

0.48

0.50

0.52

0.54

0.56

0.58

0.60

0.62

0.64

0.66

0.68

0.70

0.72

0.74

0.76

0.78

0.80

0.82

0.84

0.86

0.88

0.90

0.92

0.94

0.96

0.98
0.2

0.000

0.012

0.025

0.039

0.054

0.070

0.088

0.106

0.125

0.246

0.169

0.187

0.205

0.224

0.242

0.261

0.281

0.300

0.320

0.339

0.359

0.379

0.398

0.418

0.438

0.458

0.479

0.499

0.520

0.540

0.561

0.582

0.603

0.624

0.645

0.667

0.688

0.710

0.731

0.753

0.775

0.797

0.819

0.841

0.864

0.886

0.909

0.932

0.954

0.977
0.4

0.000

0.006

0.012

0.018

0.025

0.032

0.038

0.047

0.056

0.064

0.073

0.083

0.179

0.200

0.220

0.241

0.262

0.283

0.304

0.325

0.346

0.367

0.388

0.409

0.431

0.452

0.473

0.494

0.516

0.537

0.558

0.580

0.601

0.623

0.644

0.666

0.688

0.709

0.731

0.753

0.775

0.797

0.819

0.842

0.864

0.886

0.909

0.931

0.954

0.977
Homologous Parameter

$\begin{array}{lllll}0.6 & 0.8 & 1.0 & 1.2 & 1.4 \\ 0.000 & 0.000 & 0.000 & 0.000 & 0.000 \\ 0.006 & 0.005 & 0.005 & 0.005 & 0.005 \\ 0.012 & 0.011 & 0.010 & 0.011 & 0.011 \\ 0.018 & 0.018 & 0.016 & 0.016 & 0.017 \\ 0.025 & 0.024 & 0.021 & 0.022 & 0.023 \\ 0.032 & 0.031 & 0.027 & 0.028 & 0.029 \\ 0.039 & 0.039 & 0.034 & 0.035 & 0.036 \\ 0.047 & 0.046 & 0.041 & 0.041 & 0.043 \\ 0.055 & 0.055 & 0.050 & 0.048 & 0.050 \\ 0.064 & 0.063 & 0.058 & 0.056 & 0.057 \\ 0.073 & 0.072 & 0.068 & 0.063 & 0.065 \\ 0.082 & 0.082 & 0.078 & 0.071 & 0.073 \\ 0.092 & 0.092 & 0.088 & 0.080 & 0.082 \\ 0.150 & 0.102 & 0.099 & 0.089 & 0.091 \\ 0.175 & 0.121 & 0.110 & 0.099 & 0.101 \\ 0.200 & 0.142 & 0.124 & 0.111 & 0.111 \\ 0.224 & 0.165 & 0.140 & 0.127 & 0.124 \\ 0.243 & 0.191 & 0.159 & 0.150 & 0.138 \\ 0.266 & 0.219 & 0.181 & 0.168 & 0.154 \\ 0.291 & 0.249 & 0.206 & 0.188 & 0.182 \\ 0.315 & 0.279 & 0.234 & 0.211 & 0.202 \\ 0.340 & 0.307 & 0.265 & 0.237 & 0.224 \\ 0.365 & 0.329 & 0.297 & 0.266 & 0.250 \\ 0.389 & 0.357 & 0.330 & 0.299 & 0.279 \\ 0.413 & 0.385 & 0.359 & 0.333 & 0.311 \\ 0.437 & 0.413 & 0.384 & 0.366 & 0.345 \\ 0.461 & 0.440 & 0.415 & 0.392 & 0.379 \\ 0.484 & 0.467 & 0.446 & 0.424 & 0.405 \\ 0.507 & 0.493 & 0.475 & 0.457 & 0.440 \\ 0.530 & 0.518 & 0.504 & 0.489 & 0.474 \\ 0.553 & 0.543 & 0.532 & 0.520 & 0.5508 \\ 0.575 & 0.568 & 0.559 & 0.549 & 0.540 \\ 0.598 & 0.592 & 0.585 & 0.577 & 0.570 \\ 0.620 & 0.615 & 0.610 & 0.604 & 0.599 \\ 0.642 & 0.639 & 0.635 & 0.631 & 0.627 \\ 0.664 & 0.662 & 0.659 & 0.656 & 0.653 \\ 0.686 & 0.684 & 0.682 & 0.680 & 0.679 \\ 0.708 & 0.707 & 0.706 & 0.704 & 0.704 \\ 0.730 & 0.730 & 0.729 & 0.728 & 0.728 \\ 0.753 & 0.752 & 0.751 & 0.751 & 0.751 \\ 0.775 & 0.774 & 0.774 & 0.774 & 0.774 \\ 0.797 & 0.797 & 0.796 & 0.797 & 0.797 \\ 0.819 & 0.819 & 0.819 & 0.8199 & 0.819 \\ 0.841 & 0.841 & 0.841 & 0.841 & 0.842 \\ 0.864 & 0.864 & 0.864 & 0.864 & 0.864 \\ 0.886 & 0.886 & 0.886 & 0.886 & 0.886 \\ 0.909 & 0.909 & 0.909 & 0.909 & 0.909 \\ 0.931 & 0.931 & 0.931 & 0.931 & 0.931 \\ 0.954 & 0.954 & 0.954 & 0.954 & 0.954 \\ 0.977 & 0.977 & 0.977 & 0.977 & 0.977\end{array}$


5.

SRPRP Head and Torque Characteristics Under Steam/Water and Steam/Air/Water Conditions for Both AC and DC Motor Operations without Diffuser Section (Tasks $4,8,11$ and 12)

5.1 Theoretical Basis

The theory for determining the performance of the pump operating under the steam/water two-phase condition has been developed for the Electric Power Research Institute and described in one of the EPRI reports (EPRI NP-5529SP(November, 1987)). The basic difference of these formula from those of the air/water two-phase flow case are as follows:

i) The mass conservation equations for the gas phase and liquid phase have the source (or sink) terms due to the condensation and evaporation effects of the flow media.

ii) The energy equation is needed to define the energy balance between two phases, or equivalently to determine the amount of the condensation or evaporation.

iii) The table of enthalpy for the steam and liquid phases is needed for actual computations.

These formula have been used many times to date to determine the characteristics of the condensable two-phase flow pumps and provided good correlation with experimental data. One of such comparisons has recently been made when the MIST pump was tested by Babcock \& Wilcox (see B\&W Report No. NRC-04-83-168/RP 2399-1, January 1988).

One of the major differences in the present SRPRP case from the PWR (pressurized water reactor) primary coolant pump is that the line static pressure of the former is $1 / 3000$ to $1 / 50$ that of the latter. More specifically, the range of the line pressures to be investigated is from $0.05 \mathrm{~atm}(=0.7 \mathrm{psi})$ to $3 \mathrm{~atm}$ (= 44 psi) compared to, e.g., 2500 psi of the PWR. The gradient of the enthalpy of the water (or the heavy water in this case) is extremely large and the density of the steam is very small. This sometimes causes the instability for the numerical calculations. A slight change of the static pressure generates an enormous amount of vapor or water through vaporization or condensation process, depending on the sign of the pressure gradient. Therefore, although the governing equations remain the same, the numerical computations become totally different at such low static pressure case. As a matter of fact, the computation becomes extremely unstable. 
Let us explain the problem described above in more specific terms; two key quantities at these low line pressures different from those of the PWR cases are the steam density and the $d h / d p$ where $h$ is the saturated enthalpy and $p$ is the static pressure as is shown in Table below.

\begin{tabular}{|c|c|c|}
\hline & PWR & SRP \\
\hline $\begin{array}{c}\text { Steam Density } \\
\text { (1bm/cubic feet) }\end{array}$ & 7.65 & (at $\mathrm{p}=0.7 \mathrm{psi})$ \\
\hline $\mathrm{dh} / \mathrm{dp}$ & $\mathrm{A}$ & 0.0037 \\
\hline
\end{tabular}

The amount of condensation of steam is proportional to $\mathrm{dh} / \mathrm{dp} / \mathrm{P}_{\mathrm{g}}$ as can be seen from the mass conservation equation (see Eqn. (2), p2-1, of EPRI NP-5529SP) where $\rho_{c}$ is the steam density. Therefore, that of the SRP cases becomes 7.65/0.0037 $\times(10 \times A / A)=20,675$ times that of the PWR cases where $A$ is a representative constant, as shown in Table above. Motor Operation---Without Diffuser

\subsubsection{Flow Parameters}

The single-phase pump H-Q curve, the range of line static pressure, homologous flow parameter and inlet void fraction used for these computations are the same as those used for the air/water cases (refer to Section 3.3.1 for the AC motor case and Section 4.3.1 for the DC motor case). However, for the sake of convenience, these parameters are listed herein again.

\section{1) Pump}

The SRPR pump is a double suction, double-volute centrifugal pump manufactured by Bingham Corp. having the rated flow rate of $25,000 \mathrm{gpm}(12,500 \mathrm{gpm} /$ volute) with the total head of 420 feet at the rated rotational speed of 1,000 rpm. The specific speed $N_{8}$ is 1,205 in the English units,

$$
5-2
$$


indicating that the pump is a typical centrifugal pump:

$$
\begin{aligned}
N_{1} & =N Q^{1 / 2} / H^{3 / 4} \\
& =1,000 \times 12,500^{1 / 2} / 420^{3 / 4}=1,205
\end{aligned}
$$

The head vs. flow rate curve driven by the $\mathrm{AC}$ motor at $1,000 \mathrm{rpm}$ is given in Figure 3.3.1-1.

On the other hand, when this pump is operated by the DC motor at the rotation speed of $300 \mathrm{rpm}$, the flow rate is $6,200 \mathrm{gpm}(3,100 \mathrm{gpm} /$ volute $)$.

The head vs. flow rate curve driven by the $A C$ motor at 1,000 rpm and by the DC motor at $300 \mathrm{rpm}$ are given in Figures 4.3.1-1 and 4.3.1-2, respectively. In these figures both the total pump dynamic head and that of fluid flowing are given for the AC motor pump performance while only the latter is given for the DC motor case. The correction is thus to be made for the DC motor head curve as follows:

$$
\begin{gathered}
H_{1 \phi D C}=47.7-0.4289 \times(\mathrm{Q} / 1000)^{0.943}+\Delta H \\
\Delta H=4.236 \times(\mathrm{Q} / 10,000)^{2}
\end{gathered}
$$

where the formula for Equations (5.3.1-1) and (5.3.1-2) were given by the report No. DPS-83-282 and the Los Alamos National Laboratory. Therefore, the rated head at the DC motor operation will be readily calculated

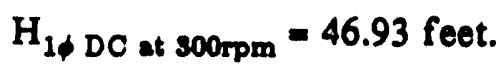

It should be noted that the homologous flow parameters and head data presented hereafter for the DC motor operation are all normalized by using $N_{R s 00}=300 \mathrm{rpm}, Q_{R s 00}=6200 \mathrm{gpm}$ and $H_{R s 00}=46.93$ feet.

The pump performance curves nondimensionalized by using the unified rated parameters (i.e., $N_{R}$, $Q_{R}$ and $H_{R}$ at $1,000 \mathrm{rpm}$ ) show a clear difference between the $A C$ and $D C$ motor operations(see Figure 4.3.1-3)).

$$
5-3
$$


2) Line Static Pressure $\left(p_{1}\right)$

The following line static pressures were used for the present calculations:

$$
\begin{aligned}
& 0.05 \mathrm{~atm} \\
& 1.00 \mathrm{~atm} \\
& 2.00 \mathrm{~atm} \\
& 3.00 \mathrm{~atm}
\end{aligned}
$$

3) Flow Rate ( Q ) and Pump Rotational Speed (N)

Since the pump performance only depends on the ratio between the flow rate and the rotational speed, the range of these parameters is expressed in terms of the homologous flow parameter defined by $\nu$ $/ \alpha_{N}$ where

$$
\begin{gathered}
\nu=Q / Q_{R} \\
\alpha_{N}=N / N_{R} .
\end{gathered}
$$

The range of the homologous parameter used for the calculations is

$$
\begin{array}{r}
\nu / \alpha_{N}= \\
0.4 \\
0.6 \\
0.8 \\
1.0 \\
1.2 \\
1.4
\end{array}
$$

With these homologous parameters, the range of the actual flow rate will be $10,000 \mathrm{gpm}$ to 35,000 gpm assuming the constant rotational speed (i.e., 1,000 rpm).

4) Inlet Void Fraction $\left(\alpha_{1}\right)$

$$
\alpha_{1}=0.00 \text { to } 0.98
$$




\section{2 .2}

Properties of $\mathrm{D}_{2} \mathrm{O}$

The steam and liquid densities and enthalpies of $\mathrm{D}_{2} \mathrm{O}$ were provided by the Los Alamos National Laboratory and a partial table of these quantities is given in Table 5.2.1.

\subsubsection{Numerical Results for AC and DC Motor Operations}

Figures 5.2.1 through 5.2.4 show the homologous head parameters as functions of the inlet void fraction and the homologous flow parameter for the static line pressures 3,2,1 and 0,05 atms, respectively, for the SRPRP operating with the AC motor, whereas Figures 5.2.5 through 5.2.8 show the same for the DC motor operation. Tables 5.2.2 through 5.2.5 show the detailed results corresponding to the AC operation, and Tables 5.2.6 through 5.2.9 show the same corresponding to the DC motor operation. Each set of the tables (i.e., for each static line pressure case) comprises the homologous head parameters, the static pressure rise, the normalized relative velocities of water and steam and the void fraction all at exit of the blade.

Although the results for the AC motor operation cases seem to be reasonable in terms of the numerical stability, those of the DC motor cases indicate a strong numerical instability, particularly for lower static cases as has been discussed in Section 5.1. Such instability is seen even in Figure 5.2 .5 at $\nu / \alpha_{N}$ $=0.6$; at a certain $\alpha_{1}$ the calculated homologous head suddenly deviates from a smooth curve. This instability becomes so violent in the case of $p_{1}=2$ atm where the homologous head shoots out of the scale of the graph.

Substantial effort has been made in finding the exact cause of this numerical instability problem, including 1) change of the number of control points along the stream tube of the blade flow passage and 2) change of the method of solving the system of the nonlinear ordinary differential equations from the Runge-Kutta method (i.e., an "explicit" method) to the Gear's method (i.e., an "implicit method").

The reason for using the Gear's method is that the implicit method is said to be more stable even for the "stiff" differential equations which seem to be the case.

Despite such effort, the numerical instability problem existed in the present numerical computations, requiring further study possibly by using a super computer in which the increment size and truncation errors will be eliminated from the possible cause of the instability problem. Fixing this numerical

$$
5-5
$$


instability problem will become imperative if the present two-phase flow pump computer code is to be incorporated into a system code such as TRAC code for the PWR coolant system safety analysis.

The torque calculations (Tasks 9 and 10) would be made based on the results of computations by using the computer code specially written for that purpose. However, due to the reasons described above, such torque computations have not been presently carried out. 
Properties of saturated $D_{2} \mathrm{O}$

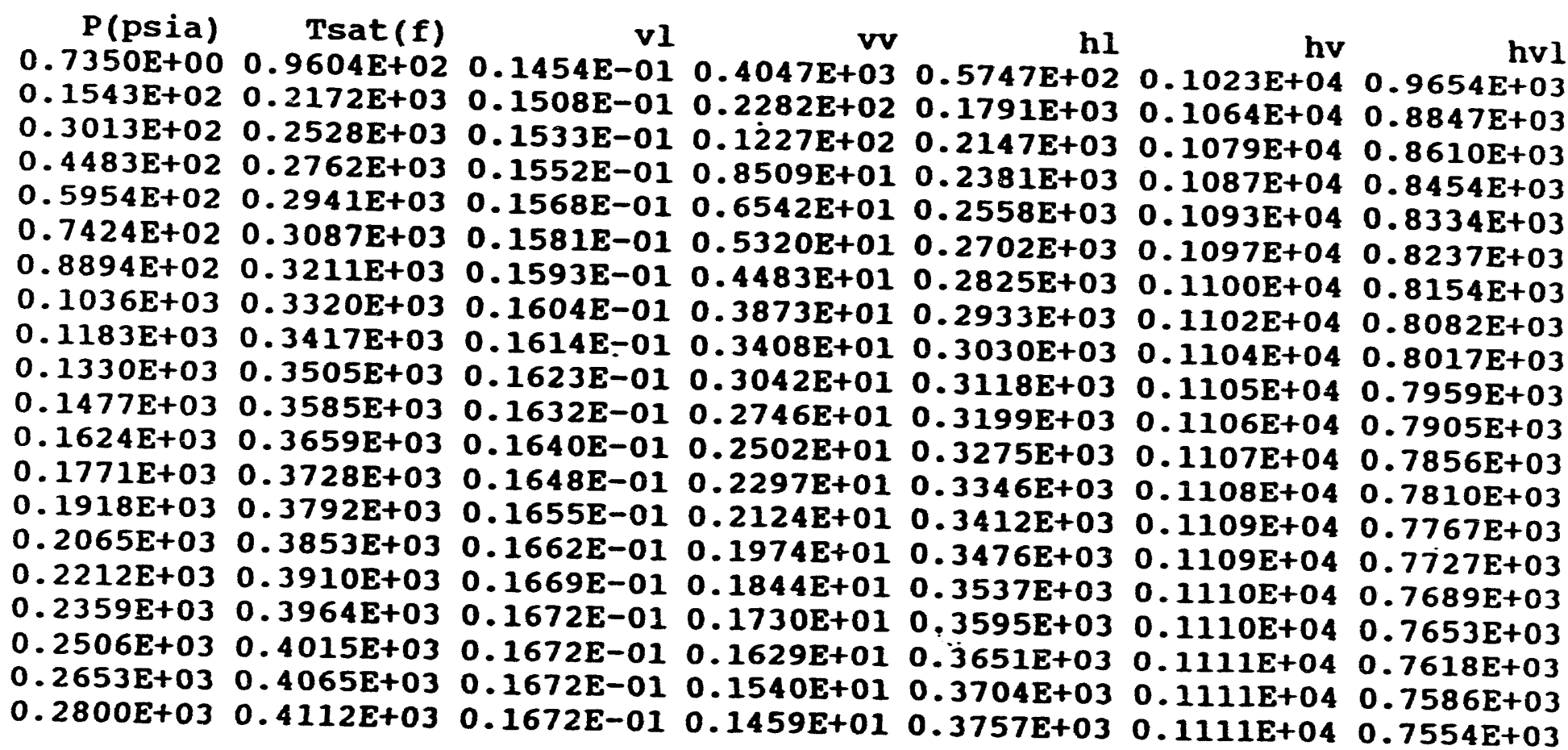

Unit for $v l$ and $v v$ [ft*t3/1bm] $h l$ and hv [btu/lom] 


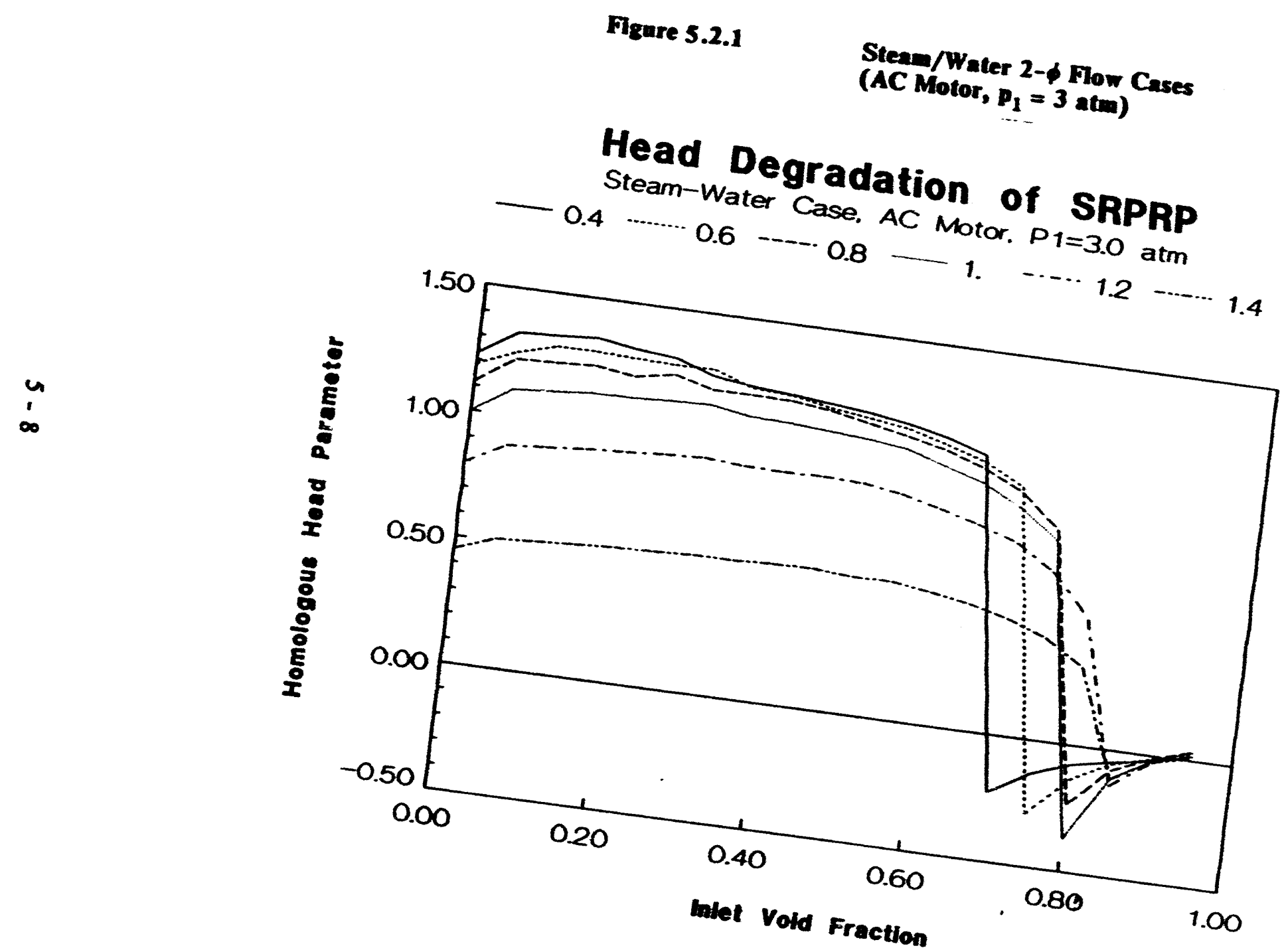


Figure 5.2.2

Steam/Wat or 2- $\$$ Flow Cases

(AC Motor, $p_{1}=2$ atw)

Head Degradation of SRPRP

Steam-Water Case. AC Motor. P1=2.0 atm
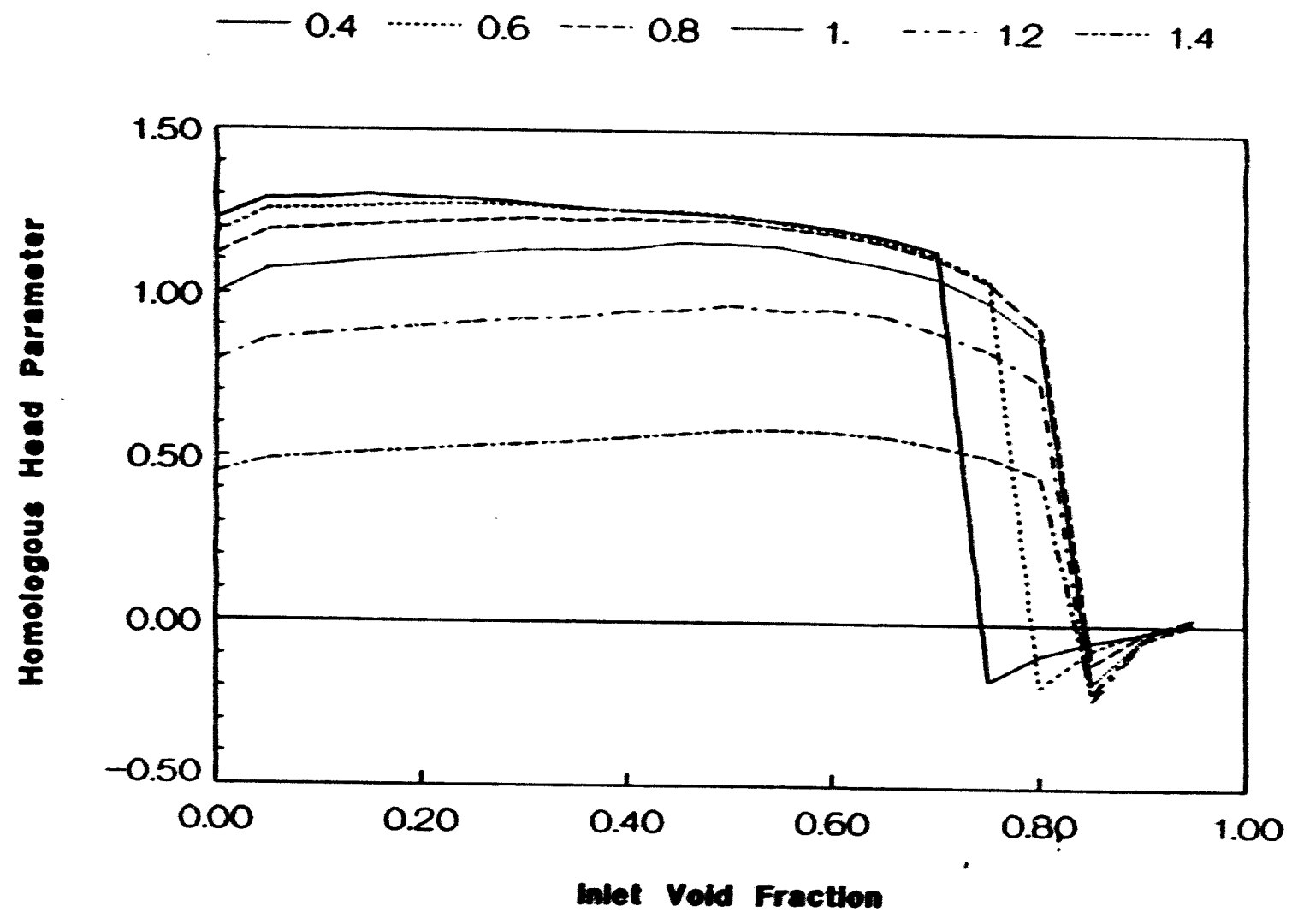
Fizure 5.2.3 Stean/Water 2-\$ Fow Cases (AC Motor, $p_{1}=1$ atw)

\section{Head Degradation of SRPRP}

Steam-Water Case. AC Motor. $P_{1}=1.0 \mathrm{~atm}$

Figure 5.2.4

Stean/Water 2- $\$$ Flow Case (AC Motor, $p_{1}=0.05 \mathrm{~atm}$ )

\section{Head Degradation of SRPRP}

Steam-Water Case. AC Motor. $P_{1}=0.05 \mathrm{~atm}$
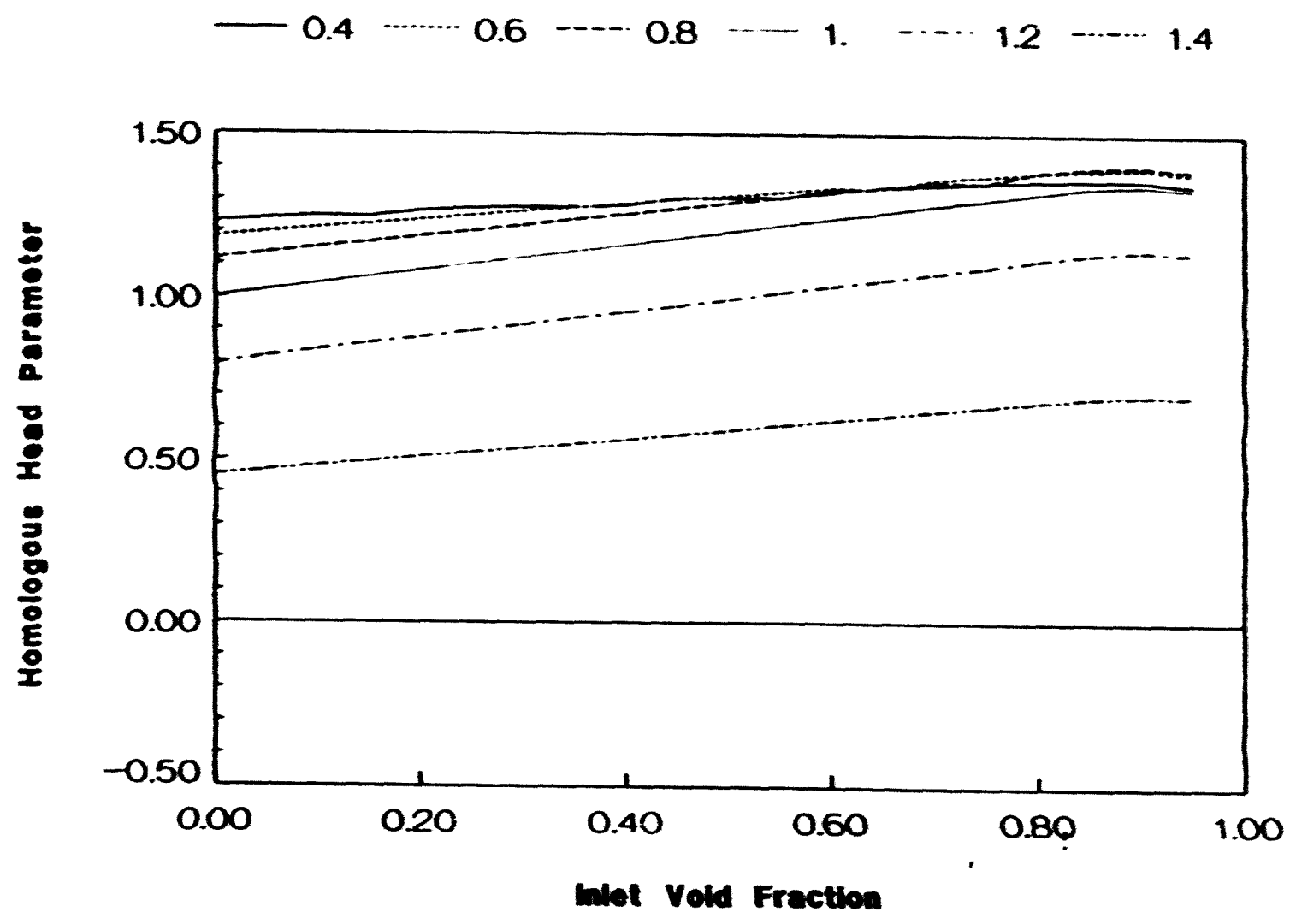
Table 5.2 .2

Steam/Water 2- $\phi$ Flow Cases

(AC Motor, $p_{j}=3 \mathrm{~atm}$ )

Homologous Head Parameter ve. Inlet Vold Fraction for Various Homologous Flow Parameters at $P 1=3.0 \mathrm{~atm}$. AC Motor Operation

\begin{tabular}{|l|r|r|r|r|r|r|}
\hline $\begin{array}{l}\text { Inlet } \\
\text { Vold } \\
\text { Fraction }\end{array}$ & \multicolumn{7}{|c|}{ Homologous Flow Parameter } \\
\cline { 2 - 7 } & 0.4 & 0.6 & 0.8 & 1.0 & 1.2. & 1.4 \\
\hline 0.00 & 1.228 & 1.184 & 1.117 & 1.000 & 0.794 & 0.452 \\
0.05 & 1.327 & 1.250 & 1.223 & 1.103 & 0.883 & 0.507 \\
0.10 & 1.334 & 1.292 & 1.226 & 1.115 & 0.895 & 0.517 \\
0.15 & 1.500 & 1.293 & 1.236 & 1.128 & 0.908 & 0.526 \\
0.20 & 1.321 & 1.286 & 1.214 & 1.131 & 0.917 & 0.534 \\
0.25 & 1.307 & 1.280 & 1.242 & 1.140 & 0.927 & 0.540 \\
0.30 & 1.406 & 1.284 & 1.202 & 1.143 & 0.934 & 0.547 \\
0.35 & 1.236 & 1.228 & 1.201 & 1.118 & 0.923 & 0.546 \\
0.40 & 1.224 & 1.219 & 1.200 & 1.115 & 0.920 & 0.554 \\
0.45 & 1.208 & 1.192 & 1.181 & 1.104 & 0.924 & 0.556 \\
0.50 & 1.193 & 1.173 & 1.152 & 1.091 & 0.919 & 0.545 \\
0.55 & 1.171 & 1.153 & 1.123 & 1.073 & 0.896 & 0.547 \\
0.60 & 1.140 & 1.113 & 1.090 & 1.026 & 0.859 & 0.525 \\
0.65 & 1.092 & 1.064 & 1.039 & 0.983 & 0.816 & 0.501 \\
0.70 & -0.225 & 0.986 & 0.966 & 0.908 & 0.767 & 0.462 \\
0.75 & -0.133 & -0.291 & 0.838 & 0.795 & 0.674 & 0.408 \\
0.80 & -0.079 & -0.134 & -0.231 & -0.367 & 0.502 & 0.313 \\
0.85 & -0.046 & -0.055 & -0.081 & -0.114 & -0.138 & -0.115 \\
0.90 & -0.021 & -0.011 & -0.012 & -0.019 & -0.028 & -0.026 \\
0.95 & 0.019 & 0.032 & 0.036 & 0.034 & 0.023 & 0.008 \\
\hline
\end{tabular}


Table 5.2 .2

Steam/Water 2- $\phi$ Flow Casos (AC Motor, $p_{1}=3 \mathrm{~atm}$ )

(contlaued)

Static Pressure Rise in pela at Vane Exit ve. Inlet Vold Fraction for Various Homologous Flow Parameters at P1 $=3.0 \mathrm{~atm}$.

AC Motor Operation

\begin{tabular}{|l|r|r|r|r|r|r|}
\hline \multirow{2}{*}{$\begin{array}{l}\text { Inlet } \\
\text { Vold } \\
\text { Fraction }\end{array}$} & \multicolumn{7}{|c|}{ Homologous 1 10w Parameter } \\
\cline { 2 - 7 } & 0.4 & 0.6 & 0.8 & 1.0 & 1.2 & 1.4 \\
\hline 0.00 & 162.54 & 163.31 & 164.40 & 165.79 & 167.49 & 169.51 \\
0.05 & 187.09 & 179.03 & 189.26 & 191.11 & 193.34 & 196.04 \\
0.10 & 189.45 & 189.02 & 189.17 & 192.91 & 195.53 & 198.98 \\
0.15 & 230.58 & 189.56 & 191.26 & 195.35 & 198.08 & 201.87 \\
0.20 & 188.62 & 188.58 & 185.95 & 195.46 & 199.78 & 205.01 \\
0.25 & 187.14 & 188.02 & 192.99 & 197.64 & 202.19 & 206.91 \\
0.30 & 213.41 & 190.45 & 184.21 & 198.46 & 203.98 & 209.59 \\
0.35 & 175.07 & 179.21 & 185.32 & 192.87 & 200.58 & 208.39 \\
0.40 & 176.07 & 179.88 & 187.07 & 193.50 & 200.62 & 213.08 \\
0.45 & 176.76 & 177.21 & 185.68 & 193.07 & 203.66 & 215.30 \\
0.50 & 179.22 & 177.93 & 182.90 & 193.23 & 204.94 & 212.24 \\
0.55 & 181.63 & 179.96 & 181.95 & 193.92 & 202.54 & 216.93 \\
0.60 & 184.34 & 179.77 & 182.37 & 189.64 & 198.12 & 212.06 \\
0.65 & 186.30 & 180.54 & 182.03 & 189.60 & 195.20 & 209.25 \\
0.70 & 1.52 & 179.72 & 181.30 & 186.38 & 195.37 & 203.85 \\
0.75 & 0.94 & 2.07 & 175.44 & 181.47 & 190.08 & 198.84 \\
0.80 & 0.61 & 1.08 & 1.79 & 2.84 & 174.94 & 187.18 \\
0.85 & 0.42 & 0.60 & 0.84 & 1.13 & 1.45 & 1.83 \\
0.90 & 0.34 & 0.39 & 0.45 & 0.53 & 0.60 & 0.68 \\
0.95 & 0.30 & 0.31 & 0.32 & 0.33 & 0.35 & 0.36 \\
\hline
\end{tabular}


Table $\mathbf{3 . 2 . 2}$

Steam/Water 2- $\phi$ Flow Casos
(AC Motor, $p_{2}=3$ atm)
(Contlaued)

Normalized Relative Velocity of Water at Vane Exit ve. Inlet Vold Fraction for Varlous Homologous Flow Parameters at PI = 3.0 atm. AC Motor Operation

\begin{tabular}{|l|l|l|l|l|l|l|}
\hline \multirow{2}{*}{$\begin{array}{l}\text { Inlet } \\
\text { Vold } \\
\text { Fraction }\end{array}$} & \multicolumn{6}{|c|}{ Homologous Flow Parameter } \\
\cline { 2 - 7 } & 0.4 & 0.6 & 0.8 & 1.0 & 1.2 & 1.4 \\
\hline 0.00 & 0.112 & 0.167 & 0.222 & 0.278 & 0.334 & 0.389 \\
0.05 & 0.106 & 0.159 & 0.211 & 0.264 & 0.317 & 0.370 \\
0.10 & 0.100 & 0.150 & 0.200 & 0.250 & 0.300 & 0.350 \\
0.15 & 0.095 & 0.142 & 0.189 & 0.236 & 0.284 & 0.331 \\
0.20 & 0.089 & 0.134 & 0.178 & 0.223 & 0.267 & 0.312 \\
0.25 & 0.083 & 0.125 & 0.167 & 0.209 & 0.250 & 0.292 \\
0.30 & 0.078 & 0.117 & 0.156 & 0.195 & 0.234 & 0.273 \\
0.35 & 0.072 & 0.109 & 0.145 & 0.181 & 0.217 & 0.253 \\
0.40 & 0.067 & 0.100 & 0.134 & 0.167 & 0.200 & 0.234 \\
0.45 & 0.061 & 0.092 & 0.123 & 0.153 & 0.184 & 0.214 \\
0.50 & 0.056 & 0.084 & 0.111 & 0.139 & 0.167 & 0.195 \\
0.55 & 0.050 & 0.075 & 0.100 & 0.125 & 0.150 & 0.176 \\
0.60 & 0.045 & 0.067 & 0.089 & 0.112 & 0.134 & 0.156 \\
0.65 & 0.039 & 0.059 & 0.078 & 0.098 & 0.117 & 0.137 \\
0.70 & 0.842 & 0.050 & 0.067 & 0.084 & 0.100 & 0.117 \\
0.75 & 0.860 & 0.838 & 0.056 & 0.070 & 0.084 & 0.098 \\
0.80 & 0.872 & 0.867 & 0.862 & 0.856 & 0.067 & 0.078 \\
0.85 & 0.881 & 0.884 & 0.890 & 0.901 & 0.917 & 0.935 \\
0.90 & 0.887 & 0.894 & 0.906 & 0.922 & 0.942 & 0.966 \\
0.95 & 0.890 & 0.900 & 0.914 & 0.932 & 0.954 & 0.979 \\
\hline
\end{tabular}


Table 5.2 .2

Steam/Water 2- $\phi$ Flow Cases

(AC Motor, $p_{1}=3 \mathrm{~atm}$ )

(continued)

Normalized Relative Velocity of steam at Vane Exit vs. Inlet Void Fraction for Various Homologous Flow Parameters at $P 1=3.0 \mathrm{~atm}$.

AC Motor Operation

\begin{tabular}{|l|l|l|l|l|l|l|}
\hline \multirow{2}{*}{$\begin{array}{l}\text { Inlet } \\
\text { Void } \\
\text { Fraction }\end{array}$} & \multicolumn{6}{|c|}{ Homologous Flow Parameter } \\
\cline { 2 - 7 } & 0.4 & 0.6 & 0.8 & 1.0 & 1.2 & 1.4 \\
\hline 0.00 & 0.000 & 0.000 & 0.000 & 0.000 & 0.000 & 0.000 \\
0.05 & 0.000 & 0.000 & 0.000 & 0.000 & 0.000 & 0.000 \\
0.10 & 0.000 & 0.000 & 0.000 & 0.000 & 0.000 & 0.000 \\
0.15 & 0.000 & 0.000 & 0.000 & 0.000 & 0.000 & 0.000 \\
0.20 & 0.000 & 0.000 & 0.000 & 0.000 & 0.000 & 0.000 \\
0.25 & 0.000 & 0.000 & 0.000 & 0.000 & 0.000 & 0.000 \\
0.30 & 0.000 & 0.000 & 0.000 & 0.000 & 0.000 & 0.000 \\
0.45 & 0.000 & 0.000 & 0.000 & 0.000 & 0.000 & 0.000 \\
0.50 & 0.000 & 0.000 & 0.000 & 0.000 & 0.000 & 0.000 \\
0.55 & 0.000 & 0.000 & 0.000 & 0.000 & 0.000 & 0.000 \\
0.60 & 0.000 & 0.000 & 0.000 & 0.000 & 0.000 & 0.000 \\
0.65 & 0.000 & 0.000 & 0.000 & 0.000 & 0.000 & 0.000 \\
0.70 & 0.031 & 0.000 & 0.000 & 0.000 & 0.000 & 0.000 \\
0.75 & 0.059 & 0.048 & 0.000 & 0.000 & 0.000 & 0.000 \\
0.80 & 0.077 & 0.102 & 0.109 & 0.084 & 0.000 & 0.000 \\
0.85 & 0.088 & 0.130 & 0.168 & 0.200 & 0.226 & 0.245 \\
0.90 & 0.097 & 0.146 & 0.195 & 0.243 & 0.291 & 0.338 \\
0.95 & 0.105 & 0.158 & 0.211 & 0.264 & 0.318 & 0.371 \\
\hline
\end{tabular}


Table 5.2.9

Steam/Water 2- $\phi$ Flow Cases

(DC Motor, $p_{1}=0.05$ atm)

(continued)

Static Pressure Rise in psia at Vane Exit vs. Inlet Void Fraction for Various Homologous Flow Parameters at $\mathrm{Pl}=0.05 \mathrm{~atm}$.

DC Motor Operation

\begin{tabular}{|l|r|r|r|r|r|r|}
\hline \multirow{2}{*}{$\begin{array}{l}\text { Inlet } \\
\text { Void } \\
\text { Fraction }\end{array}$} & \multicolumn{6}{|c|}{ Homologous Flow Parameter } \\
\cline { 2 - 7 } & 0.4 & 0.6 & 0.8 & 1.0 & 1.2 & 1.4 \\
\hline 0.00 & 15.58 & 15.63 & 15.70 & 15.79 & 15.91 & 16.04 \\
0.05 & 15.96 & 16.04 & 16.11 & 14.10 & 16.43 & 16.62 \\
0.10 & 15.98 & 16.08 & 16.19 & 15.88 & 16.57 & 16.83 \\
0.15 & 15.99 & 16.11 & 16.26 & 16.31 & 16.70 & 16.99 \\
0.20 & 16.02 & 16.15 & 16.32 & 16.51 & 16.83 & 17.16 \\
0.25 & 16.02 & 16.17 & 16.37 & 16.61 & 16.50 & 17.32 \\
0.30 & 16.04 & 16.20 & 16.43 & 16.70 & 16.96 & 17.46 \\
0.45 & 16.28 & 16.48 & 16.70 & 17.10 & 17.54 & 17.57 \\
0.50 & 16.42 & 16.63 & 16.92 & 17.25 & 16.80 & 17.96 \\
0.55 & 16.59 & 16.82 & 17.12 & 17.51 & 17.99 & 18.56 \\
0.60 & 16.81 & 17.02 & 17.35 & 17.76 & 18.26 & 18.85 \\
0.65 & 17.08 & 17.20 & 17.60 & 18.03 & 18.57 & 19.18 \\
0.70 & 17.43 & 17.38 & 17.71 & 18.29 & 18.90 & 19.56 \\
0.75 & 17.91 & 17.72 & 18.03 & 18.36 & 19.26 & 19.97 \\
0.80 & 18.64 & 18.12 & 18.27 & 18.68 & 19.19 & 20.17 \\
0.85 & 19.86 & 18.91 & 18.61 & 18.74 & 19.41 & 20.15 \\
0.90 & 22.30 & 20.67 & 19.83 & 19.64 & 19.76 & 20.22 \\
0.95 & 29.64 & 0.00 & 0.00 & 0.00 & 0.00 & 0.00 \\
\hline
\end{tabular}


Table 5.2.9

Steam/Water 2- $\phi$ Flow Cases

(DC Motor, $p_{1}=0.05 \mathrm{~atm}$ )

(continued)

Normalized Relative Velocity of Water at Vane Exit vs. Inlet Void Fraction for Various Homologous Flow Parameters at $P I=0.05$ atm. DC Motor Operation

\begin{tabular}{|l|l|l|l|l|l|l|}
\hline \multirow{2}{*}{$\begin{array}{l}\text { Inlet } \\
\text { Void } \\
\text { Fraction }\end{array}$} & \multicolumn{5}{|c|}{ Homologous Flow Parameter } \\
\cline { 2 - 7 } & 0.4 & 0.6 & 0.8 & 1.0 & 1.2 & 1.4 \\
\hline 0.00 & 0.092 & 0.138 & 0.184 & 0.230 & 0.276 & 0.322 \\
0.05 & 0.087 & 0.131 & 0.175 & 0.218 & 0.262 & 0.306 \\
0.10 & 0.083 & 0.124 & 0.166 & 0.207 & 0.248 & 0.290 \\
0.15 & 0.078 & 0.117 & 0.156 & 0.195 & 0.234 & 0.273 \\
0.20 & 0.073 & 0.110 & 0.147 & 0.184 & 0.221 & 0.257 \\
0.25 & 0.069 & 0.103 & 0.138 & 0.172 & 0.207 & 0.241 \\
0.30 & 0.064 & 0.097 & 0.129 & 0.161 & 0.193 & 0.225 \\
0.45 & 0.050 & 0.076 & 0.101 & 0.126 & 0.152 & 0.177 \\
0.50 & 0.046 & 0.069 & 0.092 & 0.115 & 0.138 & 0.161 \\
0.55 & 0.041 & 0.062 & 0.083 & 0.103 & 0.124 & 0.145 \\
0.60 & 0.037 & 0.055 & 0.073 & 0.092 & 0.110 & 0.129 \\
0.65 & 0.032 & 0.048 & 0.064 & 0.080 & 0.097 & 0.113 \\
0.70 & 0.028 & 0.041 & 0.055 & 0.069 & 0.083 & 0.097 \\
0.75 & 0.023 & 0.034 & 0.046 & 0.057 & 0.069 & 0.080 \\
0.80 & 0.018 & 0.028 & 0.037 & 0.046 & 0.055 & 0.064 \\
0.85 & 0.014 & 0.021 & 0.028 & 0.034 & 0.041 & 0.048 \\
0.90 & 0.009 & 0.014 & 0.018 & 0.023 & 0.028 & 0.032 \\
0.95 & 0.005 & 0.895 & 0.905 & 0.917 & 0.932 & 0.949 \\
\hline
\end{tabular}


Table 5.2.9

Steam/Water 2- $\phi$ Flow Cases

(DC Motor, $p_{1}=0.05 \mathrm{~atm}$ )

(continued)

Normalized Relative Velocity of steam at Vane Exit vs. Inlet Void Fraction for Various Homologous Flow Parameters at PI $=0.05$ atm. DC Motor Operation

\begin{tabular}{|l|l|l|l|l|l|l|}
\hline \multirow{2}{*}{$\begin{array}{l}\text { Inlet } \\
\text { Void } \\
\text { Fraction }\end{array}$} & \multicolumn{5}{|c|}{ Homologous Flow Parameter } \\
\cline { 2 - 7 } & 0.4 & 0.6 & 0.8 & 1.0 & 1.2 & 1.4 \\
\hline 0.00 & 0.000 & 0.000 & 0.000 & 0.000 & 0.000 & 0.000 \\
0.05 & 0.000 & 0.000 & 0.000 & 0.000 & 0.000 & 0.000 \\
0.10 & 0.000 & 0.000 & 0.000 & 0.000 & 0.000 & 0.000 \\
0.15 & 0.000 & 0.000 & 0.000 & 0.000 & 0.000 & 0.000 \\
0.20 & 0.000 & 0.000 & 0.000 & 0.000 & 0.000 & 0.000 \\
0.25 & 0.000 & 0.000 & 0.000 & 0.000 & 0.000 & 0.000 \\
0.30 & 0.000 & 0.000 & 0.000 & 0.000 & 0.000 & 0.000 \\
0.45 & 0.000 & 0.000 & 0.000 & 0.000 & 0.000 & 0.000 \\
0.50 & 0.000 & 0.000 & 0.000 & 0.000 & 0.000 & 0.000 \\
0.55 & 0.000 & 0.000 & 0.000 & 0.000 & 0.000 & 0.000 \\
0.60 & 0.000 & 0.000 & 0.000 & 0.000 & 0.000 & 0.000 \\
0.65 & 0.000 & 0.000 & 0.000 & 0.000 & 0.000 & 0.000 \\
0.70 & 0.000 & 0.000 & 0.000 & 0.000 & 0.000 & 0.000 \\
0.75 & 0.000 & 0.000 & 0.000 & 0.000 & 0.000 & 0.000 \\
0.80 & 0.000 & 0.000 & 0.000 & 0.000 & 0.000 & 0.000 \\
0.85 & 0.000 & 0.000 & 0.000 & 0.000 & 0.000 & 0.000 \\
0.90 & 0.000 & 0.000 & 0.000 & 0.000 & 0.000 & 0.000 \\
0.95 & 0.000 & 0.120 & 0.153 & 0.179 & 0.197 & 0.206 \\
\hline
\end{tabular}


Table 5.2.9

Steam/Water 2- $\phi$ Flow Cases (DC Motor, $p_{1}=0.05 \mathrm{~atm}$ ) (continued)

Discharge Void Fraction vs. Inlet Void Fraction for Various Homologous Flow Parameters at P1 $=0.05 \mathrm{~atm}$. DC Motor Operation

\begin{tabular}{|l|l|l|l|l|l|l|}
\hline \multirow{2}{*}{$\begin{array}{l}\text { Inlet } \\
\text { Void } \\
\text { Fraction }\end{array}$} & \multicolumn{6}{|c|}{ Homologous Flow Parameter } \\
\cline { 2 - 6 } & 0.4 & 0.6 & 0.8 & 1.0 & 1.2 & 1.4 \\
\hline 0.00 & 0.000 & 0.000 & 0.000 & 0.000 & 0.000 & 0.000 \\
0.05 & 0.000 & 0.000 & 0.000 & 0.000 & 0.000 & 0.000 \\
0.10 & 0.000 & 0.000 & 0.000 & 0.000 & 0.000 & 0.000 \\
0.15 & 0.000 & 0.000 & 0.000 & 0.000 & 0.000 & 0.000 \\
0.20 & 0.000 & 0.000 & 0.000 & 0.000 & 0.000 & 0.000 \\
0.25 & 0.000 & 0.000 & 0.000 & 0.000 & 0.000 & 0.000 \\
0.30 & 0.000 & 0.000 & 0.000 & 0.000 & 0.000 & 0.000 \\
0.45 & 0.000 & 0.000 & 0.000 & 0.000 & 0.000 & 0.000 \\
0.50 & 0.000 & 0.000 & 0.000 & 0.000 & 0.000 & 0.000 \\
0.55 & 0.000 & 0.000 & 0.000 & 0.000 & 0.000 & 0.000 \\
0.60 & 0.000 & 0.000 & 0.000 & 0.000 & 0.000 & 0.000 \\
0.65 & 0.000 & 0.000 & 0.000 & 0.000 & 0.000 & 0.000 \\
0.70 & 0.000 & 0.000 & 0.000 & 0.000 & 0.000 & 0.000 \\
0.75 & 0.000 & 0.000 & 0.000 & 0.000 & 0.000 & 0.000 \\
0.80 & 0.000 & 0.000 & 0.000 & 0.000 & 0.000 & 0.000 \\
0.85 & 0.000 & 0.000 & 0.000 & 0.000 & 0.000 & 0.000 \\
0.90 & 0.000 & 0.000 & 0.000 & 0.000 & 0.000 & 0.000 \\
0.95 & 0.000 & 0.992 & 0.990 & 0.987 & 0.985 & 0.983 \\
\hline
\end{tabular}


Table 5.2.2

Steam/Water 2- $\phi$ Flow Cases

(AC Motor, $p_{1}=3$ atm)

(continued)

Discharge Void Fraction vs. Inlet Void Fraction

for Various Homologous Flow Parameters at PI $=3.0$ atm.

AC Motor Operation

\begin{tabular}{|l|l|l|l|l|l|l|}
\hline \multirow{2}{*}{$\begin{array}{l}\text { Inlet } \\
\text { Void } \\
\text { Fraction }\end{array}$} & \multicolumn{6}{|c|}{ Homologous Flow Parameter } \\
\cline { 2 - 7 } & 0.4 & 0.6 & 0.8 & 1.0 & 1.2 & 1.4 \\
\hline 0.00 & 0.000 & 0.000 & 0.000 & 0.000 & 0.000 & 0.000 \\
0.05 & 0.000 & 0.000 & 0.000 & 0.000 & 0.000 & .0 .000 \\
0.10 & 0.000 & 0.000 & 0.000 & 0.000 & 0.000 & 0.000 \\
0.15 & 0.000 & 0.000 & 0.000 & 0.000 & 0.000 & 0.000 \\
0.20 & 0.000 & 0.000 & 0.000 & 0.000 & 0.000 & 0.000 \\
0.25 & 0.000 & 0.000 & 0.000 & 0.000 & 0.000 & 0.000 \\
0.30 & 0.000 & 0.000 & 0.000 & 0.000 & 0.000 & 0.000 \\
0.45 & 0.000 & 0.000 & 0.000 & 0.000 & 0.000 & 0.000 \\
0.50 & 0.000 & 0.000 & 0.000 & 0.000 & 0.000 & 0.000 \\
0.55 & 0.000 & 0.000 & 0.000 & 0.000 & 0.000 & 0.000 \\
0.60 & 0.000 & 0.000 & 0.000 & 0.000 & 0.000 & 0.000 \\
0.65 & 0.000 & 0.000 & 0.000 & 0.000 & 0.000 & 0.000 \\
0.70 & 0.960 & 0.000 & 0.000 & 0.000 & 0.000 & 0.000 \\
0.75 & 0.968 & 0.950 & 0.000 & 0.000 & 0.000 & 0.000 \\
0.80 & 0.974 & 0.961 & 0.948 & 0.935 & 0.000 & 0.000 \\
0.85 & 0.981 & 0.972 & 0.962 & 0.954 & 0.945 & 0.937 \\
0.90 & 0.987 & 0.981 & 0.975 & 0.970 & 0.965 & 0.960 \\
0.95 & 0.994 & 0.991 & 0.988 & 0.985 & 0.983 & 0.980 \\
\hline
\end{tabular}


Table 5.2.3

Steam/Water 2- $\phi$ Flow Cases (AC Motor, $p_{1}=2 \mathrm{~atm}$ )

Homologous Head Parameter vs. Inlet Void Fraction for Various Homologous Flow Parameters at P1 $=2.0 \mathrm{~atm}$.

AC Motor Operation

\begin{tabular}{|l|r|r|r|r|r|r|}
\hline \multirow{2}{*}{$\begin{array}{l}\text { Inlet } \\
\text { Vold } \\
\text { Fraction }\end{array}$} & \multicolumn{7}{|c|}{ Homologous Flow Parameter } \\
\cline { 2 - 7 } & 0.4 & 0.6 & 0.8 & 1.0 & $1.2 .$. & \multicolumn{1}{c|}{1.4} \\
\hline 0.00 & 1.228 & 1.184 & 1.117 & 1.000 & 0.794 & 0.452 \\
0.05 & 1.275 & 1.257 & 1.192 & 1.074 & 0.859 & 0.493 \\
0.10 & 1.293 & 1.261 & 1.202 & 1.086 & 0.874 & 0.504 \\
0.15 & 1.304 & 1.268 & 1.211 & 1.100 & 0.888 & 0.514 \\
0.20 & 1.290 & 1.274 & 1.221 & 1.113 & 0.902 & 0.524 \\
0.25 & 1.292 & 1.277 & 1.227 & 1.123 & 0.914 & 0.534 \\
0.30 & 1.284 & 1.274 & 1.233 & 1.134 & 0.925 & 0.542 \\
0.35 & 1.267 & 1.260 & 1.228 & 1.136 & 0.931 & 0.551 \\
0.40 & 1.258 & 1.261 & 1.233 & 1.139 & 0.951 & 0.562 \\
0.45 & 1.253 & 1.259 & 1.229 & 1.160 & 0.952 & 0.574 \\
0.50 & 1.242 & 1.250 & 1.229 & 1.158 & 0.971 & 0.584 \\
0.55 & 1.228 & 1.223 & 1.209 & 1.149 & 0.956 & 0.587 \\
0.60 & 1.208 & 1.202 & 1.190 & 1.119 & 0.959 & 0.582 \\
0.65 & 1.181 & 1.172 & 1.162 & 1.094 & 0.941 & 0.570 \\
0.70 & 1.139 & 1.226 & 1.117 & 1.059 & 0.892 & 0.544 \\
0.75 & -0.172 & 1.053 & 1.045 & 0.987 & 0.836 & 0.512 \\
0.80 & -0.092 & -0.189 & 0.910 & 0.872 & 0.741 & 0.455 \\
0.85 & -0.049 & -0.072 & -0.117 & -0.176 & -0.227 & -0.206 \\
0.90 & -0.023 & -0.017 & -0.022 & -0.033 & -0.044 & -0.039 \\
0.95 & 0.004 & 0.017 & 0.024 & 0.023 & 0.013 & 0.003 \\
\hline
\end{tabular}


Table 5.2.3

Steam/Water $2-\phi$ Flow Cases
(AC Motor, $p_{1}=2$ atm)
(continued)

Static Pressure Rise in psia at Vane Exit vs. Inlet Vold Fraction for Various Homologous Flow Parameters at $\mathrm{PI}=2.0 \mathrm{~atm}$.

AC Motor Operation

\begin{tabular}{|l|r|r|r|r|r|r|}
\hline \multirow{2}{*}{$\begin{array}{l}\text { Inlet } \\
\text { Void } \\
\text { Fraction }\end{array}$} & \multicolumn{7}{|c|}{ Homologous Flow Parameter } \\
\cline { 2 - 7 } & 0.4 & 0.6 & 0.8 & 1.0 & $\cdots 1.2$ & 1.4 \\
\hline 0.00 & 164.53 & 165.32 & 166.41 & 167.83 & 169.55 & 171.59 \\
0.05 & 180.76 & 181.98 & 183.06 & 185.06 & 187.20 & 190.23 \\
0.10 & 180.15 & 182.04 & 183.98 & 186.14 & 189.47 & 193.05 \\
0.15 & 182.91 & 183.22 & 184.82 & 187.90 & 191.56 & 195.82 \\
0.20 & 179.66 & 184.02 & 186.20 & 189.42 & 193.59 & 198.23 \\
0.25 & 180.72 & 184.46 & 186.56 & 190.52 & 195.37 & 200.96 \\
0.30 & 184.57 & 183.80 & 187.38 & 192.20 & 197.11 & 203.13 \\
0.35 & 176.73 & 180.97 & 186.06 & 191.56 & 197.52 & 205.36 \\
0.40 & 176.32 & 182.16 & 187.40 & 192.02 & 202.56 & 209.59 \\
0.45 & 177.48 & 183.32 & 187.16 & 197.54 & 202.26 & 214.53 \\
0.50 & 178.21 & 183.52 & 188.85 & 198.00 & 208.58 & 219.39 \\
0.55 & 179.40 & 180.55 & 186.90 & 197.57 & 205.21 & 221.98 \\
0.60 & 180.49 & 180.81 & 186.43 & 193.30 & 209.01 & 221.57 \\
0.65 & 182.37 & 181.12 & 186.35 & 192.69 & 208.34 & 219.30 \\
0.70 & 183.98 & 180.91 & 185.32 & 192.82 & 201.54 & 213.64 \\
0.75 & 0.89 & 179.51 & 183.15 & 188.51 & 197.81 & 210.05 \\
0.80 & 0.52 & 1.05 & 175.12 & 182.83 & 191.39 & 202.77 \\
0.85 & 0.33 & 0.52 & 0.78 & 1.12 & 1.58 & 2.22 \\
0.90 & 0.24 & 0.29 & 0.36 & 0.43 & 0.51 & 0.59 \\
0.95 & 0.21 & 0.21 & 0.22 & 0.23 & 0.24 & 0.25 \\
\hline
\end{tabular}


Table 5.2.3

Steam/Water 2- $\phi$ Flow Cases

(AC Motor, $p_{1}=2 \mathrm{~atm}$ )

(continued)

Normalized Relative Velocity of Water at Vane Exit vs. Inlet Void Fraction for Various Homologous Flow parameters at $P 1=2.0$ atm.

AC Motor Operation

\begin{tabular}{|l|l|l|l|l|l|l|}
\hline \multirow{2}{*}{$\begin{array}{l}\text { Inlet } \\
\text { Vold } \\
\text { Fraction }\end{array}$} & \multicolumn{5}{|c|}{ Homologous Flow Parameter } \\
\cline { 2 - 7 } & 0.4 & 0.6 & 0.8 & 1.0 & 1.2 & 1.4 \\
\hline 0.00 & 0.111 & 0.167 & 0.222 & 0.278 & 0.334 & 0.389 \\
0.05 & 0.106 & 0.159 & 0.211 & 0.264 & 0.317 & 0.370 \\
0.10 & 0.100 & 0.150 & 0.200 & 0.250 & 0.300 & 0.350 \\
0.15 & 0.095 & 0.142 & 0.189 & 0.236 & 0.284 & 0.331 \\
0.20 & 0.089 & 0.134 & 0.178 & 0.223 & 0.267 & 0.312 \\
0.25 & 0.083 & 0.125 & 0.167 & 0.209 & 0.250 & 0.292 \\
0.30 & 0.078 & 0.117 & 0.156 & 0.195 & 0.234 & 0.273 \\
0.35 & 0.072 & 0.108 & 0.145 & 0.181 & 0.217 & 0.253 \\
0.40 & 0.067 & 0.100 & 0.134 & 0.167 & 0.200 & 0.234 \\
0.45 & 0.061 & 0.092 & 0.122 & 0.153 & 0.184 & 0.214 \\
0.50 & 0.056 & 0.083 & 0.111 & 0.139 & 0.167 & 0.195 \\
0.55 & 0.050 & 0.075 & 0.100 & 0.125 & 0.150 & 0.175 \\
0.60 & 0.045 & 0.067 & 0.089 & 0.111 & 0.134 & 0.156 \\
0.65 & 0.039 & 0.059 & 0.078 & 0.098 & 0.117 & 0.137 \\
0.70 & 0.033 & 0.050 & 0.067 & 0.084 & 0.100 & 0.117 \\
0.75 & 0.858 & 0.042 & 0.056 & 0.070 & 0.084 & 0.098 \\
0.80 & 0.872 & 0.864 & 0.045 & 0.056 & 0.067 & 0.078 \\
0.85 & 0.881 & 0.883 & 0.889 & 0.898 & 0.908 & 0.918 \\
0.90 & 0.887 & 0.894 & 0.906 & 0.922 & 0.942 & 0.965 \\
0.95 & 0.890 & 0.900 & 0.914 & 0.932 & 0.954 & 0.980 \\
\hline
\end{tabular}


Table $\mathbf{3 . 2 . 3}$

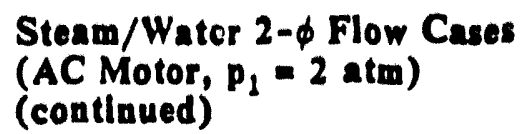

Normalized Relative Velocity of steam at Vane Exit vs. Inlet Vold Fraction for Various Homologous Flow Parameters at P1 $=2.0$ atm. AC Motor Operation

\begin{tabular}{|l|l|l|l|l|l|l|}
\hline \multirow{2}{*}{$\begin{array}{l}\text { Inlet } \\
\text { Vold } \\
\text { Fraction }\end{array}$} & \multicolumn{6}{|c|}{ Homologous Flow Parameter } \\
\cline { 2 - 7 } & 0.4 & 0.6 & 0.8 & 1.0 & 1.2 & 1.4 \\
\hline 0.00 & 0.000 & 0.000 & 0.000 & 0.000 & 0.000 & 0.000 \\
0.05 & 0.000 & 0.000 & 0.000 & 0.000 & 0.000 & .0 .000 \\
0.10 & 0.000 & 0.000 & 0.000 & 0.000 & 0.000 & 0.000 \\
0.15 & 0.000 & 0.000 & 0.000 & 0.000 & 0.000 & 0.000 \\
0.20 & 0.000 & 0.000 & 0.000 & 0.000 & 0.000 & 0.000 \\
0.25 & 0.000 & 0.000 & 0.000 & 0.000 & 0.000 & 0.000 \\
0.30 & 0.000 & 0.000 & 0.000 & 0.000 & 0.000 & 0.000 \\
0.45 & 0.000 & 0.000 & 0.000 & 0.000 & 0.000 & 0.000 \\
0.50 & 0.000 & 0.000 & 0.000 & 0.000 & 0.000 & 0.000 \\
0.55 & 0.000 & 0.000 & 0.000 & 0.000 & 0.000 & 0.000 \\
0.60 & 0.000 & 0.000 & 0.000 & 0.000 & 0.000 & 0.000 \\
0.65 & 0.000 & 0.000 & 0.000 & 0.000 & 0.000 & 0.000 \\
0.70 & 0.000 & 0.000 & 0.000 & 0.000 & 0.000 & 0.000 \\
0.75 & 0.037 & 0.000 & 0.000 & 0.000 & 0.000 & 0.000 \\
0.80 & 0.067 & 0.070 & 0.000 & 0.000 & 0.000 & 0.000 \\
0.85 & 0.085 & 0.120 & 0.144 & 0.154 & 0.143 & 0.097 \\
0.90 & 0.056 & 0.143 & 0.189 & 0.234 & 0.277 & 0.318 \\
0.95 & 0.104 & 0.157 & 0.209 & 0.262 & 0.315 & 0.368 \\
\hline
\end{tabular}


Table 5.2.3

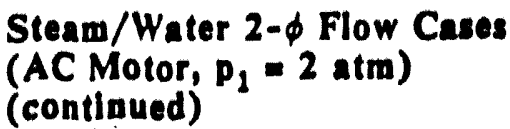

Discharge Void Fraction Vs. Inlet Vold Fraction for Various Homologous Flow Parameters at $\mathrm{PI}=2.0 \mathrm{~atm}$. AC Motor Operation

\begin{tabular}{|l|l|l|l|l|l|l|}
\hline \multirow{2}{*}{$\begin{array}{l}\text { Inlet } \\
\text { Vold } \\
\text { Fraction }\end{array}$} & \multicolumn{6}{|c|}{ Homologous Flow Parameter } \\
\cline { 2 - 7 } & 0.4 & 0.6 & 0.8 & 1.0 & 1.2 & 1.4 \\
\hline 0.00 & 0.000 & 0.000 & 0.000 & 0.000 & 0.000 & 0.000 \\
0.05 & 0.000 & 0.000 & 0.000 & 0.000 & 0.000 & 0.000 \\
0.10 & 0.000 & 0.000 & 0.000 & 0.000 & 0.000 & 0.000 \\
0.15 & 0.000 & 0.000 & 0.000 & 0.000 & 0.000 & 0.000 \\
0.20 & 0.000 & 0.000 & 0.000 & 0.000 & 0.000 & 0.000 \\
0.25 & 0.000 & 0.000 & 0.000 & 0.000 & 0.000 & 0.000 \\
0.30 & 0.000 & 0.000 & 0.000 & 0.000 & 0.000 & 0.000 \\
0.45 & 0.000 & 0.000 & 0.000 & 0.000 & 0.000 & 0.000 \\
0.50 & 0.000 & 0.000 & 0.000 & 0.000 & 0.000 & 0.000 \\
0.55 & 0.000 & 0.000 & 0.000 & 0.000 & 0.000 & 0.000 \\
0.60 & 0.000 & 0.000 & 0.000 & 0.000 & 0.000 & 0.000 \\
0.65 & 0.000 & 0.000 & 0.000 & 0.000 & 0.000 & 0.000 \\
0.70 & 0.000 & 0.000 & 0.000 & 0.000 & 0.000 & 0.000 \\
0.75 & 0.968 & 0.000 & 0.000 & 0.000 & 0.000 & 0.000 \\
0.80 & 0.974 & 0.961 & 0.000 & 0.000 & 0.000 & 0.000 \\
0.85 & 0.981 & 0.972 & 0.962 & 0.953 & 0.945 & 0.936 \\
0.90 & 0.987 & 0.981 & 0.975 & 0.970 & 0.965 & 0.960 \\
0.95 & 0.994 & 0.991 & 0.988 & 0.985 & 0.983 & 0.980 \\
\hline
\end{tabular}


Table $\mathbf{5 . 2 . 4}$

Steam/Water 2- $\phi$ Flow Cases (AC Motor, $p_{1}=1 \mathrm{~atm}$ )

Homologous Head Parameter ve. Inlet Vold Fraction for Varlous Homologous Flow Parameters at P1 $=1.0$ atm. AC Motor Operation

\begin{tabular}{|l|r|r|r|r|r|r|}
\hline \multirow{2}{*}{$\begin{array}{l}\text { Inlet } \\
\text { Vold } \\
\text { Fraction }\end{array}$} & \multicolumn{7}{|c|}{ Homologous Flow Parameter } \\
\cline { 2 - 7 } & 0.4 & 0.6 & 0.8 & 1.0 & 1.2 & 1.4 \\
\hline 0.00 & 1.228 & 1.184 & 1.117 & 1.000 & 0.794 & 0.452 \\
0.05 & 1.265 & 1.225 & 1.162 & 1.046 & 0.837 & 0.480 \\
0.10 & 1.269 & 1.234 & 1.176 & 1.063 & 0.854 & 0.493 \\
0.15 & 1.275 & 1.244 & 1.190 & 1.078 & 0.871 & 0.505 \\
0.20 & 1.285 & 1.252 & 1.202 & 1.096 & 0.888 & 0.517 \\
0.25 & 1.286 & 1.291 & 1.215 & 1.112 & 0.904 & 0.528 \\
0.30 & 1.287 & 1.267 & 1.226 & 1.127 & 0.920 & 0.539 \\
0.35 & 1.287 & 1.266 & 1.236 & 1.140 & 0.935 & 0.550 \\
0.40 & 1.287 & 1.279 & 1.252 & 1.159 & 0.955 & 0.552 \\
0.45 & 1.288 & 1.285 & 1.265 & 1.179 & 0.973 & 0.579 \\
0.50 & 1.286 & 1.289 & 1.274 & 1.194 & 0.992 & 0.593 \\
0.55 & 1.278 & 1.288 & 1.283 & 1.205 & 1.003 & 0.604 \\
0.60 & 1.273 & 1.285 & 1.282 & 1.198 & 1.009 & 0.607 \\
0.65 & 1.264 & 1.269 & 1.271 & 1.202 & 1.017 & 0.619 \\
0.70 & 1.249 & 1.255 & 1.247 & 1.194 & 1.002 & 0.607 \\
0.75 & 1.225 & 1.231 & 1.224 & 1.165 & 0.988 & 0.601 \\
0.80 & 1.179 & 1.184 & 1.177 & 1.121 & 0.947 & 0.580 \\
0.85 & -0.068 & -0.138 & 1.083 & 1.035 & 0.881 & 0.538 \\
0.90 & -0.028 & -0.033 & -0.052 & -0.079 & -0.103 & -0.091 \\
0.95 & -0.004 & 0.007 & 0.012 & 0.010 & 0.002 & -0.005 \\
\hline
\end{tabular}


Table 5.2.4 Steam/Water 2- $\phi$ Flow Cases

(AC Motor, $\left.p_{1}=1 \mathrm{~atm}\right)$

(contlnued)

Static Pressure Rise in psia at Vane Exit vs. Inlet Void Fraction for Varlous Homologous Flow Parameters at $\mathrm{P1}=1.0 \mathrm{~atm}$.

AC Motor Operation

\begin{tabular}{|l|r|r|r|r|r|r|}
\hline \multirow{2}{*}{$\begin{array}{l}\text { Inlet } \\
\text { Vold } \\
\text { Fraction }\end{array}$} & \multicolumn{6}{|c|}{ Homologous Flow Parameter } \\
\cline { 2 - 7 } & 0.4 & 0.6 & 0.8 & 1.0 & 1.2 & 1.4 \\
\hline 0.00 & 167.36 & 168.16 & 169.27 & 170.71 & 178.46 & 174.54 \\
0.05 & 175.40 & 176.53 & 178.01 & 179.88 & 182.64 & 185.81 \\
0.10 & 175.56 & 176.99 & 178.94 & 181.50 & 184.77 & 188.25 \\
0.15 & 176.00 & 177.55 & 179.98 & 182.62 & 186.72 & 191.43 \\
0.20 & 177.68 & 178.03 & 180.82 & 184.36 & 188.82 & 194.07 \\
0.25 & 187.11 & 186.07 & 181.71 & 185.68 & 190.51 & 196.49 \\
0.30 & 176.94 & 178.90 & 182.37 & 186.89 & 192.25 & 198.17 \\
0.35 & 174.58 & 177.28 & 183.00 & 187.90 & 193.81 & 200.18 \\
0.40 & 175.94 & 179.64 & 185.26 & 190.55 & 197.20 & 197.82 \\
0.45 & 176.99 & 180.51 & 187.10 & 193.55 & 199.87 & 208.81 \\
0.50 & 177.04 & 181.28 & 188.18 & 195.61 & 203.47 & 212.88 \\
0.55 & 176.39 & 181.28 & 189.85 & 197.28 & 205.04 & 216.02 \\
0.60 & 177.25 & 181.68 & 189.99 & 194.98 & 205.43 & 215.78 \\
0.65 & 178.13 & 179.89 & 188.62 & 196.61 & 207.96 & 220.59 \\
0.70 & 179.43 & 180.49 & 185.69 & 196.60 & 204.47 & 215.17 \\
0.75 & 181.11 & 181.21 & 185.63 & 193.77 & 203.91 & 215.23 \\
0.80 & 182.09 & 181.17 & 184.59 & 191.64 & 199.80 & 212.03 \\
0.85 & 0.23 & 0.46 & 181.37 & 188.26 & 197.24 & 207.12 \\
0.90 & 0.14 & 0.19 & 0.27 & 0.36 & 0.47 & 0.61 \\
0.95 & 0.11 & 0.11 & 0.12 & 0.13 & 0.14 & 0.15 \\
\hline
\end{tabular}


Table $\mathbf{5 . 2 . 4}$

Steam/Water 2- $\phi$ Flow Cases

(AC Motor, $p_{1}=1 \mathrm{~atm}$ )

(contlaued)

Normalized Relative Velocity of Water at Vane Exit vs. Inlet Vold Fraction for Various Homologous Flow Parameters at $P 1=1.0$ atm.

AC Motor Operation

\begin{tabular}{|l|l|l|l|l|l|l|}
\hline \multirow{2}{*}{$\begin{array}{l}\text { Inlet } \\
\text { Vold } \\
\text { Fraction }\end{array}$} & \multicolumn{5}{|c|}{ Homologous Flow Parameter } \\
\cline { 2 - 7 } & 0.4 & 0.6 & 0.8 & 1.0 & 1.2 & 1.4 \\
\hline 0.00 & 0.111 & 0.167 & 0.222 & 0.278 & 0.334 & 0.389 \\
0.05 & 0.106 & 0.158 & 0.211 & 0.264 & 0.317 & 0.370 \\
0.10 & 0.100 & 0.150 & 0.200 & 0.250 & 0.300 & 0.351 \\
0.15 & 0.095 & 0.142 & 0.189 & 0.236 & 0.284 & 0.331 \\
0.20 & 0.089 & 0.133 & 0.178 & 0.222 & 0.267 & 0.311 \\
0.25 & 0.083 & 0.125 & 0.167 & 0.209 & 0.250 & 0.292 \\
0.30 & 0.078 & 0.117 & 0.156 & 0.195 & 0.234 & 0.273 \\
0.35 & 0.072 & 0.108 & 0.145 & 0.181 & 0.217 & 0.253 \\
0.40 & 0.067 & 0.100 & 0.134 & 0.167 & 0.200 & 0.234 \\
0.45 & 0.061 & 0.092 & 0.122 & 0.153 & 0.184 & 0.214 \\
0.50 & 0.056 & 0.083 & 0.111 & 0.139 & 0.167 & 0.195 \\
0.55 & 0.050 & 0.075 & 0.100 & 0.125 & 0.150 & 0.175 \\
0.60 & 0.045 & 0.067 & 0.089 & 0.111 & 0.134 & 0.156 \\
0.65 & 0.039 & 0.058 & 0.078 & 0.097 & 0.117 & 0.136 \\
0.70 & 0.033 & 0.050 & 0.067 & 0.084 & 0.100 & 0.117 \\
0.75 & 0.028 & 0.042 & 0.056 & 0.070 & 0.084 & 0.097 \\
0.80 & 0.022 & 0.033 & 0.045 & 0.056 & 0.067 & 0.078 \\
0.85 & 0.881 & 0.881 & 0.033 & 0.042 & 0.050 & 0.059 \\
0.90 & 0.887 & 0.894 & 0.905 & 0.920 & 0.939 & 0.960 \\
0.95 & 0.890 & 0.900 & 0.914 & 0.932 & 0.954 & 0.980 \\
\hline
\end{tabular}


Table 5.2.4

Steam/Water 2- $\phi$ Flow Cases

(AC Motor, $p_{1}=1 \mathrm{~atm}$ )

(continued)

Normalized Relative Velocity of steam at Vane Exit vs. Inlet Void Fraction for Various Homologous Flow Parameters at P1 $=1.0$ atm. AC Motor Operation

\begin{tabular}{|l|l|l|l|l|l|l|}
\hline \multirow{2}{*}{$\begin{array}{l}\text { Inlet } \\
\text { Void } \\
\text { Fraction }\end{array}$} & \multicolumn{5}{|c|}{ Homologous Flow Parameter } \\
\cline { 2 - 7 } & 0.4 & 0.6 & 0.8 & 1.0 & 1.2 & 1.4 \\
\hline 0.00 & 0.000 & 0.000 & 0.000 & 0.000 & 0.000 & 0.000 \\
0.05 & 0.000 & 0.000 & 0.000 & 0.000 & 0.000 & 0.000 \\
0.10 & 0.000 & 0.000 & 0.000 & 0.000 & 0.000 & 0.000 \\
0.15 & 0.000 & 0.000 & 0.000 & 0.000 & 0.000 & 0.000 \\
0.20 & 0.000 & 0.000 & 0.000 & 0.000 & 0.000 & 0.000 \\
0.25 & 0.000 & 0.000 & 0.000 & 0.000 & 0.000 & 0.000 \\
0.30 & 0.000 & 0.000 & 0.000 & 0.000 & 0.000 & 0.000 \\
0.45 & 0.000 & 0.000 & 0.000 & 0.000 & 0.000 & 0.000 \\
0.50 & 0.000 & 0.000 & 0.000 & 0.000 & 0.000 & 0.000 \\
0.55 & 0.000 & 0.000 & 0.000 & 0.000 & 0.000 & 0.000 \\
0.60 & 0.000 & 0.000 & 0.000 & 0.000 & 0.000 & 0.000 \\
0.65 & 0.000 & 0.000 & 0.000 & 0.000 & 0.000 & 0.000 \\
0.70 & 0.000 & 0.000 & 0.000 & 0.000 & 0.000 & 0.000 \\
0.75 & 0.000 & 0.000 & 0.000 & 0.000 & 0.000 & 0.000 \\
0.80 & 0.000 & 0.000 & 0.000 & 0.000 & 0.000 & 0.000 \\
0.85 & 0.069 & 0.067 & 0.000 & 0.000 & 0.000 & 0.000 \\
0.90 & 0.090 & 0.130 & 0.164 & 0.189 & 0.202 & 0.199 \\
0.95 & 0.102 & 0.154 & 0.205 & 0.256 & 0.307 & 0.357 \\
\hline
\end{tabular}


Table 5.2.4

Stea $m$ /Water 2- $\phi$ Flow Cases

(AC Motor, $p_{1} \neq 1 \mathrm{~atm}$ )

(continued)

Discharge Void Fraction vs. Inlet Void Fraction

for Various Homologous Flow Parameters at $P 1=1.0$ atm. AC Motor Operation

\begin{tabular}{|l|l|l|l|l|l|l|}
\hline \multirow{2}{*}{$\begin{array}{l}\text { Inlet } \\
\text { Void } \\
\text { Fraction }\end{array}$} & \multicolumn{6}{|c|}{ Homologous Flow Parameter } \\
\cline { 2 - 7 } & 0.4 & 0.6 & 0.8 & 1.0 & 1.2 & 1.4 \\
\hline 0.00 & 0.000 & 0.000 & 0.000 & 0.000 & 0.000 & 0.000 \\
0.05 & 0.000 & 0.000 & 0.000 & 0.000 & 0.000 & 0.000 \\
0.10 & 0.000 & 0.000 & 0.000 & 0.000 & 0.000 & 0.000 \\
0.15 & 0.000 & 0.000 & 0.000 & 0.000 & 0.000 & 0.000 \\
0.20 & 0.000 & 0.000 & 0.000 & 0.000 & 0.000 & 1.000 \\
0.25 & 0.000 & 0.000 & 0.000 & 0.000 & 0.000 & 1.000 \\
0.30 & 0.000 & 0.000 & 0.000 & 0.000 & 0.000 & 0.000 \\
0.45 & 0.000 & 0.000 & 0.000 & 0.000 & 0.000 & 0.000 \\
0.50 & 0.000 & 0.000 & 0.000 & 0.000 & 0.000 & 0.000 \\
0.55 & 0.000 & 0.000 & 0.000 & 0.000 & 0.000 & 0.000 \\
0.60 & 0.000 & 0.000 & 0.000 & 0.000 & 0.000 & 0.000 \\
0.65 & 0.000 & 0.000 & 0.000 & 0.000 & 0.000 & 0.000 \\
0.70 & 0.000 & 0.000 & 0.000 & 0.000 & 0.000 & 0.000 \\
0.75 & 0.000 & 0.000 & 0.000 & 0.000 & 0.000 & 0.000 \\
0.80 & 0.000 & 0.000 & 0.000 & 0.000 & 0.000 & 0.000 \\
0.85 & 0.981 & 0.972 & 0.000 & 0.000 & 0.000 & 0.000 \\
0.90 & 0.987 & 0.981 & 0.975 & 0.970 & 0.964 & 0.959 \\
0.95 & 0.994 & 0.991 & 0.988 & 0.985 & 0.983 & 0.980 \\
\hline
\end{tabular}


Table 5.2.5

Steam/Water 2- $\phi$ Flow Cases

(DC Motor, $p_{1}=0.05 \mathrm{~atm}$ )

Homologous Head Parameter vs. Inlet Void Fraction for Various Homologous Flow Parameters at $P 1=0.05$ atm. AC Motor Operation

\begin{tabular}{|l|l|l|l|l|l|l|}
\hline \multirow{2}{*}{$\begin{array}{l}\text { Inlet } \\
\text { Void } \\
\text { Fraction }\end{array}$} & \multicolumn{7}{|c|}{ Homologous Flow Parameter } \\
\cline { 2 - 7 } & 0.4 & 0.6 & 0.8 & 1.0 & 1.2 & 1.4 \\
\hline 0.00 & 1.228 & 1.184 & 1.117 & 1.000 & 0.794 & 0.452 \\
0.05 & 1.237 & 1.198 & 1.135 & 1.021 & 0.815 & 0.466 \\
0.10 & 1.246 & 1.211 & 1.152 & 1.041 & 0.835 & 0.481 \\
0.15 & 1.255 & 1.223 & 1.169 & 1.061 & 0.855 & 0.495 \\
0.20 & 1.263 & 1.236 & 1.186 & 1.081 & 0.875 & 0.509 \\
0.25 & 1.271 & 1.249 & 1.203 & 1.101 & 0.895 & 0.523 \\
0.30 & 1.276 & 1.262 & 1.220 & 1.121 & 0.915 & 0.537 \\
0.35 & 1.276 & 1.274 & 1.237 & 1.141 & 0.936 & 0.551 \\
0.40 & 1.291 & 1.288 & 1.254 & 1.161 & 0.956 & 0.565 \\
0.45 & 1.305 & 1.300 & 1.271 & 1.181 & 0.976 & 0.580 \\
0.50 & 1.306 & 1.313 & 1.289 & 1.201 & 0.996 & 0.594 \\
0.55 & 1.308 & 1.326 & 1.306 & 1.222 & 1.017 & 0.608 \\
0.60 & 1.330 & 1.339 & 1.323 & 1.242 & 1.037 & 0.622 \\
0.65 & 1.339 & 1.337 & 1.340 & 1.261 & 1.057 & 0.637 \\
0.70 & 1.347 & 1.365 & 1.357 & 1.282 & 1.077 & 0.651 \\
0.75 & 1.355 & 1.378 & 1.357 & 1.302 & 1.090 & 0.665 \\
0.80 & 1.362 & 1.388 & 1.391 & 1.322 & 1.118 & 0.680 \\
0.85 & 1.364 & 1.396 & 1.404 & 1.341 & 1.136 & 0.694 \\
0.90 & 1.364 & 1.399 & 1.409 & 1.348 & 1.149 & 0.702 \\
0.95 & 1.349 & 1.383 & 1.395 & 1.337 & 1.140 & 0.698 \\
\hline
\end{tabular}


Table 5.2.5

Steam/Water 2- $\phi$ Flow Cases

(DC Motor, $p_{1}=0.05$ atm)

(continued)

Static Pressure Rise in psia at Vane Exit vs. Inlet Void Fraction for Various Homologous Flow Parameters at $\mathrm{P1}=0.05 \mathrm{~atm}$.

AC Motor Operation

\begin{tabular}{|l|c|c|c|c|c|c|}
\hline \multirow{2}{*}{$\begin{array}{l}\text { Inlet } \\
\text { Void } \\
\text { Fraction }\end{array}$} & \multicolumn{6}{|c|}{ Homologous Flow Parameter } \\
\cline { 2 - 7 } & 0.4 & 0.6 & 0.8 & 1.0 & 1.2 & 1.4 \\
\hline 0.00 & 173.37 & 174.20 & 175.36 & 176.84 & 178.66 & 180.81 \\
0.05 & 173.94 & 175.09 & 176.69 & 178.76 & 181.30 & 184.30 \\
0.10 & 174.20 & 175.66 & 177.71 & 180.36 & 183.57 & 187.40 \\
0.15 & 172.10 & 176.20 & 178.67 & 181.85 & 185.74 & 190.33 \\
0.20 & 174.85 & 176.70 & 179.57 & 183.26 & 187.77 & 193.09 \\
0.25 & 174.88 & 177.19 & 180.43 & 184.59 & 189.68 & 195.70 \\
0.30 & 174.16 & 177.64 & 181.22 & 185.83 & 191.47 & 198.12 \\
0.35 & 174.16 & 178.09 & 182.00 & 187.02 & 193.17 & 200.44 \\
0.40 & 174.40 & 178.59 & 182.78 & 188.19 & 194.80 & 202.62 \\
0.45 & 175.82 & 179.02 & 183.52 & 189.29 & 196.34 & 204.66 \\
0.50 & 173.70 & 179.48 & 184.23 & 190.32 & 197.76 & 206.54 \\
0.55 & 172.76 & 179.89 & 185.12 & 191.30 & 199.10 & 208.30 \\
0.60 & 176.58 & 180.36 & 185.55 & 192.20 & 200.30 & 209.89 \\
0.65 & 176.91 & 176.93 & 186.21 & 193.11 & 201.50 & 211.41 \\
0.70 & 177.43 & 181.23 & 186.90 & 194.04 & 202.68 & 212.87 \\
0.75 & 177.77 & 181.95 & 183.41 & 194.95 & 203.85 & 214.33 \\
0.80 & 178.10 & 182.22 & 188.55 & 195.96 & 205.14 & 216.01 \\
0.85 & 177.63 & 182.18 & 188.90 & 197.07 & 206.18 & 217.64 \\
0.90 & 177.90 & 182.14 & 188.51 & 196.12 & 206.60 & 217.41 \\
0.95 & 179.25 & 182.25 & 187.68 & 195.06 & 204.25 & 215.29 \\
\hline
\end{tabular}


Table 5.2.5

Steam/Water 2- $\phi$ Flow Cases

(DC Motor, $p_{1}=0.05 \mathrm{~atm}$ )

(continued)

Normalized Relative Velocity of Water at Vane Exit vs. Inlet Void Fraction for Various Homologous Flow Parameters at $\mathrm{Pl}=0.05 \mathrm{~atm}$. AC Motor Operation

\begin{tabular}{|l|l|l|l|l|l|l|}
\hline \multirow{2}{*}{$\begin{array}{l}\text { Inlet } \\
\text { Void } \\
\text { Fraction }\end{array}$} & \multicolumn{5}{|c|}{ Homologous Flow Parameter } \\
\cline { 2 - 7 } & 0.4 & 0.6 & 0.8 & 1.0 & 1.2 & 1.4 \\
\hline 0.00 & 0.111 & 0.167 & 0.222 & 0.278 & 0.334 & $\cdots .389$ \\
0.05 & 0.106 & 0.158 & 0.211 & 0.264 & 0.317 & 0.370 \\
0.10 & 0.100 & 0.150 & 0.200 & 0.250 & 0.300 & 0.350 \\
0.15 & 0.095 & 0.142 & 0.189 & 0.236 & 0.284 & 0.331 \\
0.20 & 0.089 & 0.133 & 0.178 & 0.222 & 0.267 & 0.311 \\
0.25 & 0.083 & 0.125 & 0.167 & 0.209 & 0.250 & 0.292 \\
0.30 & 0.078 & 0.117 & 0.156 & 0.195 & 0.234 & 0.272 \\
0.35 & 0.072 & 0.108 & 0.145 & 0.181 & 0.217 & 0.253 \\
0.40 & 0.067 & 0.100 & 0.133 & 0.167 & 0.200 & 0.234 \\
0.45 & 0.061 & 0.092 & 0.122 & 0.153 & 0.184 & 0.214 \\
0.50 & 0.056 & 0.083 & 0.111 & 0.139 & 0.167 & 0.195 \\
0.55 & 0.050 & 0.075 & 0.100 & 0.125 & 0.150 & 0.175 \\
0.60 & 0.045 & 0.067 & 0.089 & 0.111 & 0.133 & 0.156 \\
0.65 & 0.039 & 0.058 & 0.078 & 0.097 & 0.117 & 0.136 \\
0.70 & 0.033 & 0.050 & 0.067 & 0.083 & 0.100 & 0.117 \\
0.75 & 0.028 & 0.042 & 0.056 & 0.070 & 0.085 & 0.097 \\
0.80 & 0.022 & 0.033 & 0.045 & 0.056 & 0.067 & 0.080 \\
0.85 & 0.017 & 0.025 & 0.033 & 0.042 & 0.050 & 0.058 \\
0.90 & 0.011 & 0.017 & 0.022 & 0.028 & 0.033 & 0.039 \\
0.95 & 0.006 & 0.008 & 0.011 & 0.014 & 0.017 & 0.019 \\
\hline
\end{tabular}


Table 5.2.5

Steam/Water 2- $\phi$ Flow Cases (DC Motor, $p_{1}=0.05 \mathrm{~atm}$ )

(continued)

Normalized Relative Velocity of steam at Vane Exit vs. Inlet Void Fraction for Various Homologous Flow Parameters at P1 $=0.05$ atm. AC Motor Operation

\begin{tabular}{|l|l|l|l|l|l|l|}
\hline \multirow{2}{*}{$\begin{array}{l}\text { Inlet } \\
\text { Void } \\
\text { Fraction }\end{array}$} & \multicolumn{5}{|c|}{ Homologous Flow Parameter } \\
\cline { 2 - 6 } & 0.4 & 0.6 & 0.8 & 1.0 & 1.2 & 1.4 \\
\hline 0.00 & 0.000 & 0.000 & 0.000 & 0.000 & 0.000 & 0.000 \\
0.05 & 0.000 & 0.000 & 0.000 & 0.000 & 0.000 & 0.000 \\
0.10 & 0.000 & 0.000 & 0.000 & 0.000 & 0.000 & 0.000 \\
0.15 & 0.000 & 0.000 & 0.000 & 0.000 & 0.000 & 0.000 \\
0.20 & 0.000 & 0.000 & 0.000 & 0.000 & 0.000 & 0.000 \\
0.25 & 0.000 & 0.000 & 0.000 & 0.000 & 0.000 & 0.000 \\
0.30 & 0.000 & 0.000 & 0.000 & 0.000 & 0.000 & 0.000 \\
0.45 & 0.000 & 0.000 & 0.000 & 0.000 & 0.000 & 0.000 \\
0.50 & 0.000 & 0.000 & 0.000 & 0.000 & 0.000 & 0.000 \\
0.55 & 0.000 & 0.000 & 0.000 & 0.000 & 0.000 & 0.000 \\
0.60 & 0.000 & 0.000 & 0.000 & 0.000 & 0.000 & 0.000 \\
0.65 & 0.000 & 0.000 & 0.000 & 0.000 & 0.000 & 0.000 \\
0.70 & 0.000 & 0.000 & 0.000 & 0.000 & 0.000 & 0.000 \\
0.75 & 0.000 & 0.000 & 0.000 & 0.000 & 0.000 & 0.000 \\
0.80 & 0.000 & 0.000 & 0.000 & 0.000 & 0.000 & 0.000 \\
0.85 & 0.000 & 0.000 & 0.000 & 0.000 & 0.000 & 0.000 \\
0.90 & 0.000 & 0.000 & 0.000 & 0.000 & 0.000 & 0.000 \\
0.95 & 0.000 & 0.000 & 0.000 & 0.000 & 0.000 & 0.000 \\
\hline
\end{tabular}


Table $\mathbf{5 . 2 . 5}$

Steam/Water 2- $\phi$ Flow Cases (DC Motor, $p_{1}=0.05 \mathrm{~atm}$ ) (continued)

Discharge Void Fraction vs. Inlet Void Fraction for Various Homologous Flow Parameters at $P I=0.05$ atm. AC Motor Operation

\begin{tabular}{|l|l|l|l|l|l|l|}
\hline \multirow{2}{*}{$\begin{array}{l}\text { Inlet } \\
\text { Void } \\
\text { Fraction }\end{array}$} & \multicolumn{6}{|c|}{ Homologous Flow Parameter } \\
\cline { 2 - 7 } & 0.4 & 0.6 & 0.8 & 1.0 & 1.2 & 1.4 \\
\hline 0.00 & 0.000 & 0.000 & 0.000 & 0.000 & 0.000 & 0.000 \\
0.05 & 0.000 & 0.000 & 0.000 & 0.000 & 0.000 & 0.000 \\
0.10 & 0.000 & 0.000 & 0.000 & 0.000 & 0.000 & 0.000 \\
0.15 & 0.000 & 0.000 & 0.000 & 0.000 & 0.000 & 0.000 \\
0.20 & 0.000 & 0.000 & 0.000 & 0.000 & 0.000 & 0.000 \\
0.25 & 0.000 & 0.000 & 0.000 & 0.000 & 0.000 & 0.000 \\
0.30 & 0.000 & 0.000 & 0.000 & 0.000 & 0.000 & 0.000 \\
0.45 & 0.000 & 0.000 & 0.000 & 0.000 & 0.000 & 0.000 \\
0.50 & 0.000 & 0.000 & 0.000 & 0.000 & 0.000 & 0.000 \\
0.55 & 0.000 & 0.000 & 0.000 & 0.000 & 0.000 & 0.000 \\
0.60 & 0.000 & 0.000 & 0.000 & 0.000 & 0.000 & 0.000 \\
0.65 & 0.000 & 0.000 & 0.000 & 0.000 & 0.000 & 0.000 \\
0.70 & 0.000 & 0.000 & 0.000 & 0.000 & 0.000 & 0.000 \\
0.75 & 0.000 & 0.000 & 0.000 & 0.000 & 0.000 & 0.000 \\
0.80 & 0.000 & 0.000 & 0.000 & 0.000 & 0.000 & 0.000 \\
0.85 & 0.000 & 0.000 & 0.000 & 0.000 & 0.000 & 0.000 \\
0.90 & 0.000 & 0.000 & 0.000 & 0.000 & 0.000 & 0.000 \\
0.95 & 0.000 & 0.000 & 0.000 & 0.000 & 0.000 & 0.000 \\
\hline
\end{tabular}




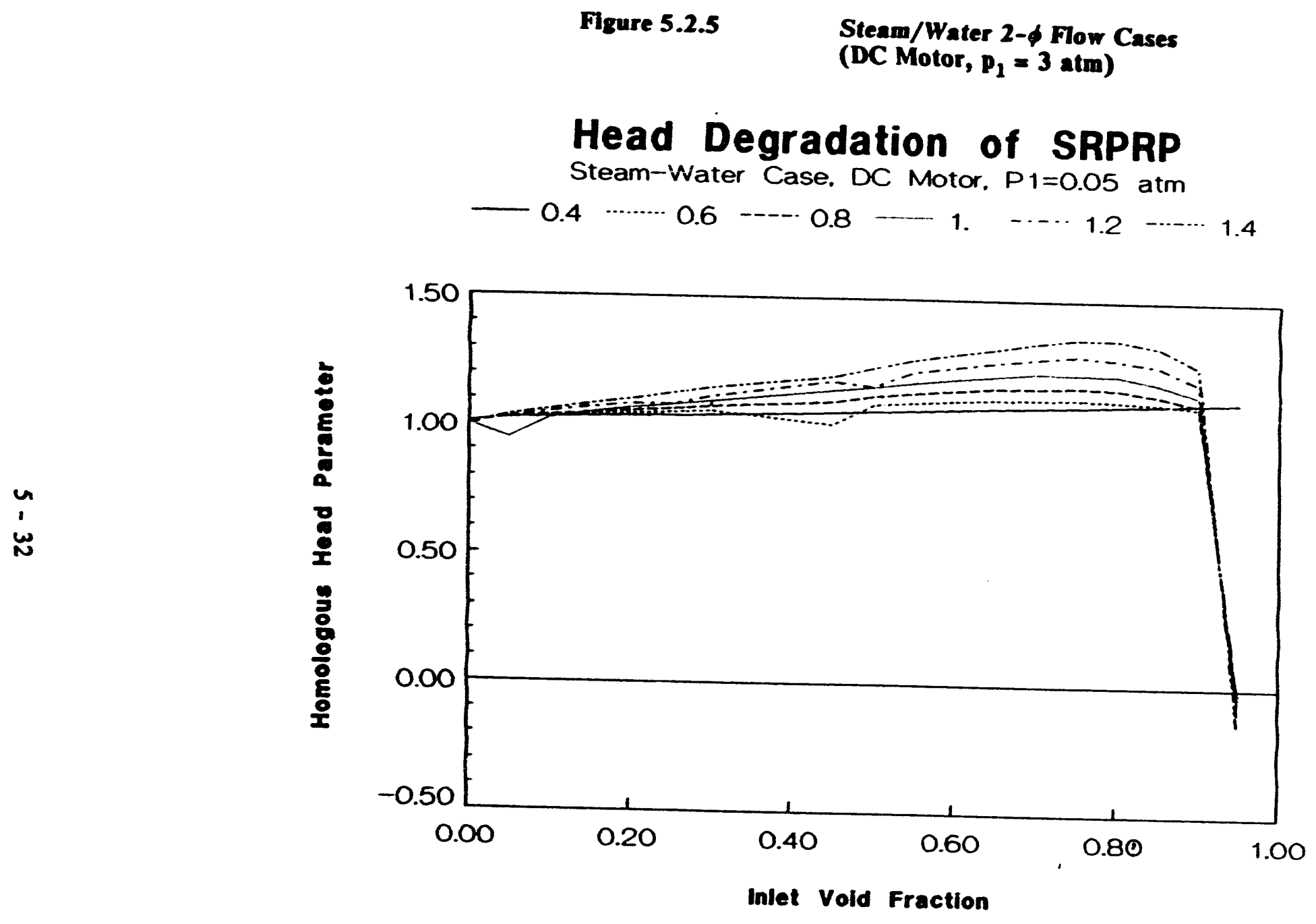


uopoest plon joju

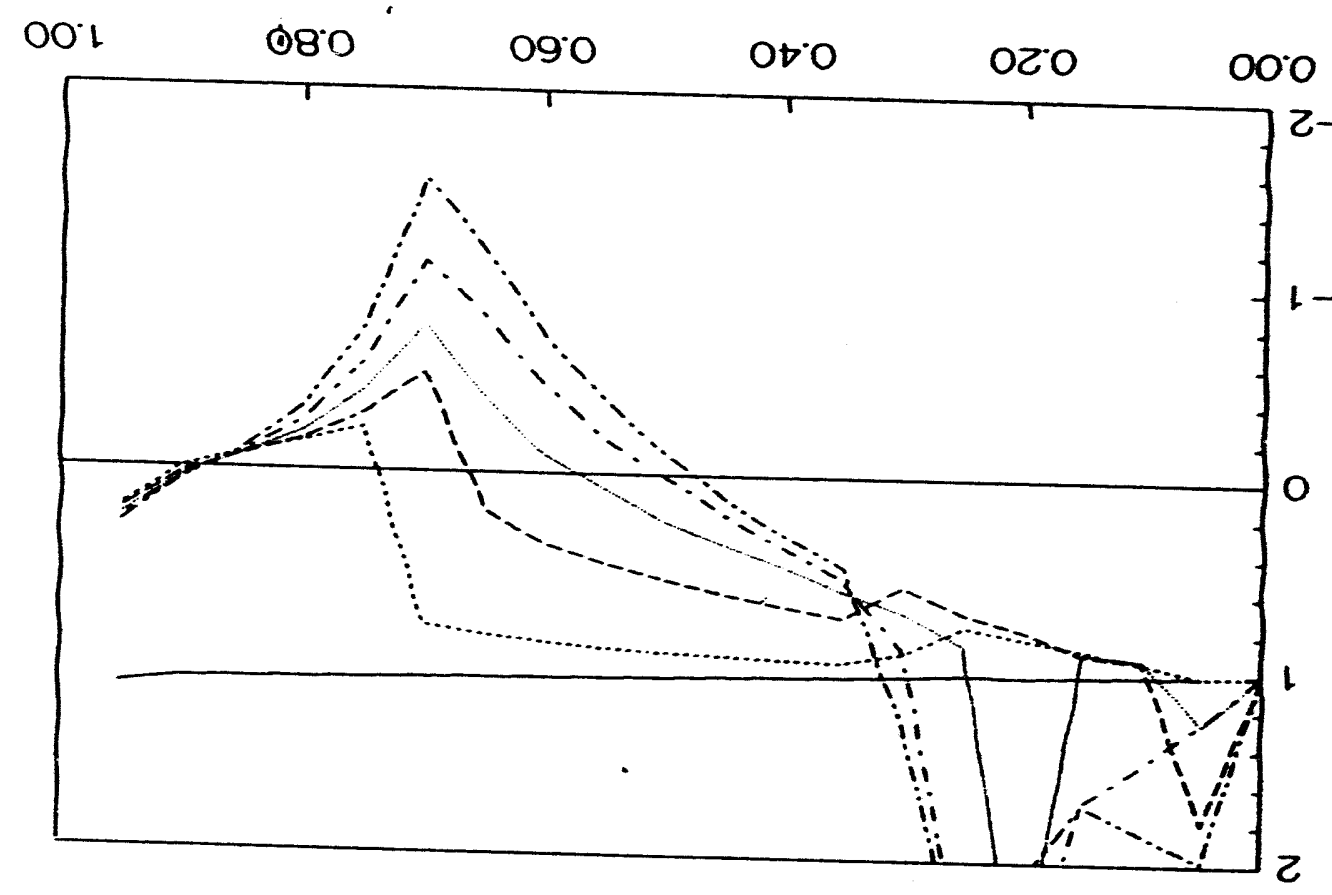



$\forall * 1$

$8^{\circ} 0$

$9^{\circ} 0$

to


dydys to uo!fepes6әа peән

(m) $\tau=I_{d}$ 'solow $\left.\supset 0\right)$ SOSE) MOLL $\phi-\tau$ JOJEM/wroIS 
Figure 5.2.7

Steam/Water 2- $\phi$ Flow Cases

(DC Motor, $p_{1}=1 \mathrm{~atm}$ )

\section{Head Degradation of SRPRP}

Steam-Water Case. DC Motor. P1=2.0 atm

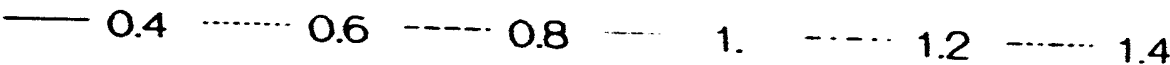

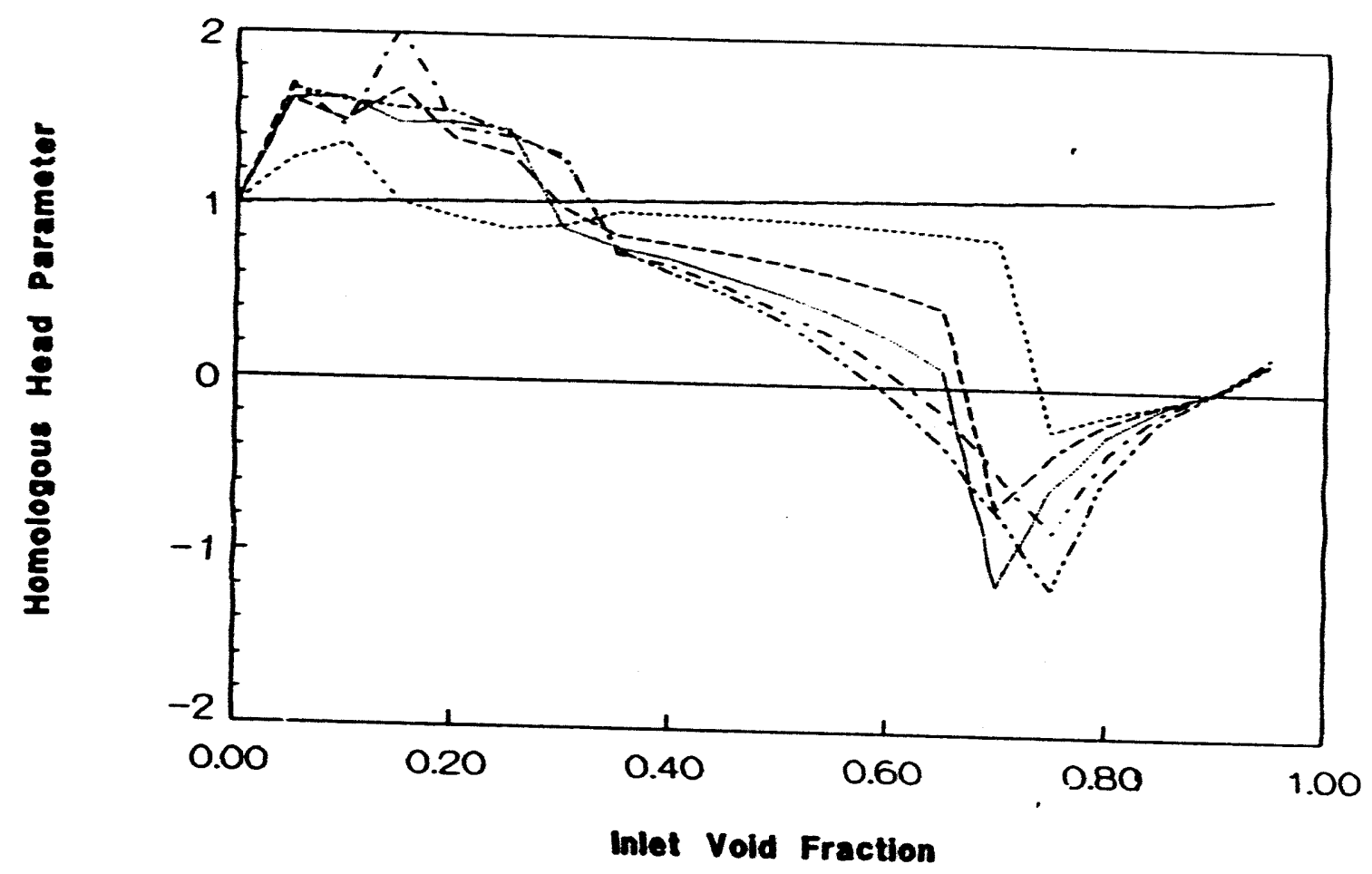




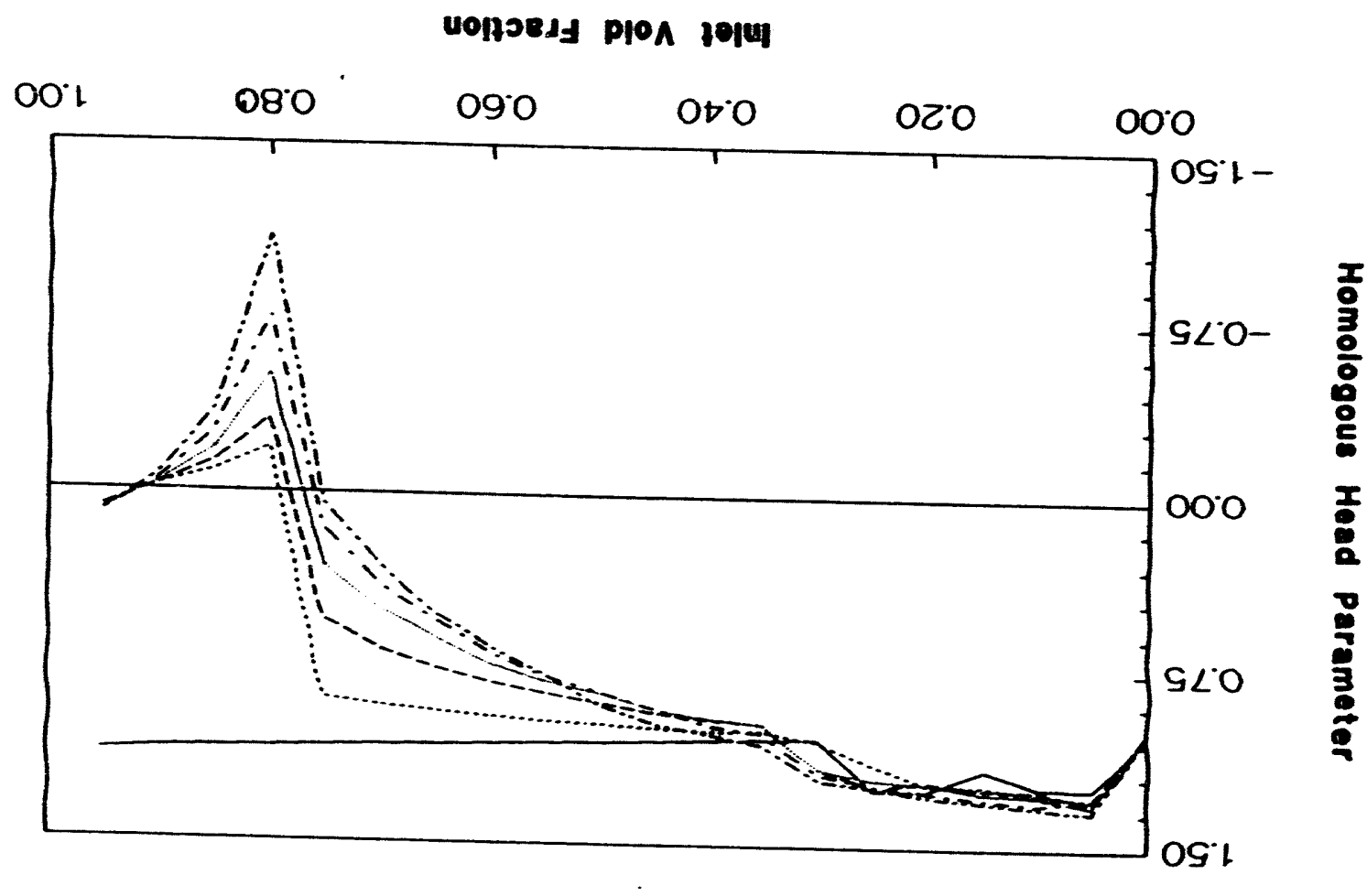



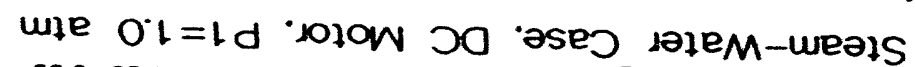 dydys to no!fepej6aq peaH

(mis SO.0 $=t_{d}$ 'solow Ja)

sases Mold $\phi-Z$ jaIs $M /$ mirals

$8^{\circ} \tau \cdot s$ asnzid 
Table 5.2.6

Steam/Water 2- $\phi$ Flow Cases (DC Motor, $p_{1}=3 \mathrm{~atm}$ )

Homologous Head Parameter vs. Inlet Void Fraction for Various Homologous Flow Parameters at $\mathrm{Pl}=3.0 \mathrm{~atm}$. DC Motor Operation

\begin{tabular}{|l|r|r|r|r|r|r|}
\hline \multirow{2}{*}{$\begin{array}{l}\text { Inlet } \\
\text { Void } \\
\text { Fraction }\end{array}$} & \multicolumn{7}{|c|}{ Homologous Flow Parameter } \\
\cline { 2 - 7 } & 0.4 & 0.6 & 0.8 & 1.0 & 1.2 & 1.4 \\
\hline 0.00 & 1.008 & 1.005 & 1.002 & 1.000 & 0.999 & 0.999 \\
0.05 & 1.013 & 1.011 & 1.791 & 1.271 & 1.260 & 1.983 \\
0.10 & 1.019 & 0.945 & 0.943 & 0.930 & & \\
0.15 & 1.024 & 0.889 & 0.910 & 0.903 & 1.692 & 1.706 \\
0.20 & 1.030 & 0.833 & 0.793 & & 2.663 & 2.726 \\
0.25 & 1.027 & 0.773 & 0.709 & 0.873 & 2.421 & 2.481 \\
0.30 & 1.042 & 0.920 & 0.572 & 0.707 & 0.910 & 1.299 \\
0.35 & 1.047 & 0.973 & 0.741 & 0.600 & 0.540 & 0.478 \\
0.40 & 1.053 & 0.966 & 0.684 & 0.479 & 0.374 & 0.303 \\
0.45 & 1.059 & 0.951 & 0.626 & 0.361 & 0.203 & 0.128 \\
0.50 & 1.065 & 0.935 & 0.556 & 0.241 & 0.017 & -0.122 \\
0.55 & 1.070 & 0.918 & 0.472 & 0.087 & -0.191 & -0.383 \\
0.60 & 1.076 & 0.893 & 0.371 & -0.097 & -0.450 & -0.714 \\
0.65 & 1.082 & 0.862 & 0.206 & -0.375 & -0.807 & -1.151 \\
0.70 & 1.087 & 0.811 & -0.516 & -0.770 & -1.105 & -1.533 \\
0.75 & 1.093 & -0.227 & -0.301 & -0.416 & -0.566 & -0.747 \\
0.80 & 1.098 & -0.146 & -0.161 & -0.200 & -0.258 & -0.331 \\
0.85 & 1.102 & -0.080 & -0.064 & -0.063 & -0.072 & -0.092 \\
0.90 & 1.107 & 0.003 & 0.033 & 0.051 & 0.059 & 0.066 \\
0.95 & 1.143 & 0.217 & 0.237 & 0.269 & 0.285 & 0.306 \\
\hline
\end{tabular}


Table 5.2.6

\author{
Steam/Water 2- $\phi$ Flow Cases \\ (DC Motor, $p_{1}=3 \mathrm{~atm}$ ) \\ (continued)
}

Static Pressure Rise in psia at Vane Exit vs. Inlet Void Fraction for Various Homologous Flow Parameters at $\mathrm{PI}=3.0 \mathrm{~atm}$. DC Motor Operation

\begin{tabular}{|l|r|r|r|r|r|r|}
\hline \multirow{2}{*}{$\begin{array}{l}\text { Inlet } \\
\text { Void } \\
\text { Fraction }\end{array}$} & \multicolumn{7}{|c|}{ Homologous Flow Parameter } \\
\cline { 2 - 7 } & 0.4 & 0.6 & 0.8 & 1.0 & 1.2 & 1.4 \\
\hline 0.00 & 14.61 & 14.66 & 14.73 & 14.81 & 14.92 & 15.04 \\
0.05 & 16.93 & 16.96 & 36.66 & 23.41 & 22.97 & 38.92 \\
0.10 & 19.51 & 17.59 & 17.65 & 17.43 & & \\
0.15 & 22.40 & 18.82 & 19.42 & 19.34 & 37.81 & 37.47 \\
0.20 & 25.65 & 20.38 & 19.47 & & 63.27 & 63.04 \\
0.25 & 29.09 & 22.30 & 20.74 & 24.91 & 60.95 & 60.85 \\
0.30 & 33.52 & 30.11 & 21.23 & 24.74 & 29.65 & 38.33 \\
0.35 & 38.36 & 36.11 & 30.04 & 26.67 & 25.50 & 24.45 \\
0.40 & 44.00 & 41.33 & 33.97 & 29.04 & 26.89 & 25.78 \\
0.45 & 50.67 & 47.38 & 38.89 & 32.51 & 29.19 & 28.09 \\
0.50 & 58.65 & 54.74 & 44.84 & 37.26 & 32.45 & 30.06 \\
0.55 & 68.40 & 63.81 & 52.18 & 42.93 & 36.96 & 33.53 \\
0.60 & 80.57 & 75.13 & 61.54 & 50.28 & 42.69 & 37.88 \\
0.65 & 96.20 & 89.72 & 72.72 & 58.84 & 49.61 & 43.30 \\
0.70 & 117.00 & 108.97 & 0.33 & 0.50 & 0.70 & 0.93 \\
0.75 & 146.05 & 0.12 & 0.19 & 0.28 & 0.37 & 0.47 \\
0.80 & 189.49 & 0.07 & 0.11 & 0.15 & 0.19 & 0.23 \\
0.85 & 261.53 & 0.04 & 0.06 & 0.07 & 0.09 & 0.11 \\
0.90 & 404.35 & 0.03 & 0.04 & 0.04 & 0.05 & 0.05 \\
0.95 & 823.48 & 0.03 & 0.03 & 0.03 & 0.03 & 0.03 \\
\hline
\end{tabular}


Table 5.2.6

Steam/Water 2- $\phi$ Flow Cases

(DC Motor, $p_{1}=3 \mathrm{~atm}$ )

(continued)

Normalized Relative Velocity of Water at Vane Exit vs. Inlet Void Fraction for Various Homologous Flow Parameters at $P 1=3.0$ atm. DC Motor Operation

\begin{tabular}{|l|l|l|l|l|l|l|}
\hline \multirow{2}{*}{$\begin{array}{l}\text { Inlet } \\
\text { Void } \\
\text { Fraction }\end{array}$} & \multicolumn{5}{|c|}{ Homologous Flow Parameter } \\
\cline { 2 - 6 } & 0.4 & 0.6 & 0.8 & 1.0 & 1.2 & 1.4 \\
\hline 0.00 & 0.092 & 0.138 & 0.184 & 0.230 & 0.276 & 0.322 \\
0.05 & 0.087 & 0.131 & 0.175 & 0.218 & 0.262 & 0.306 \\
0.10 & 0.083 & 0.124 & 0.166 & 0.207 & 0.248 & 0.290 \\
0.15 & 0.078 & 0.117 & 0.156 & 0.195 & 0.235 & 0.274 \\
0.20 & 0.073 & 0.110 & 0.147 & 0.184 & 0.221 & 0.258 \\
0.25 & 0.069 & 0.103 & 0.138 & 0.173 & 0.207 & 0.242 \\
0.30 & 0.064 & 0.097 & 0.129 & 0.161 & 0.193 & 0.226 \\
0.35 & 0.060 & 0.090 & 0.120 & 0.150 & 0.179 & 0.209 \\
0.40 & 0.055 & 0.083 & 0.110 & 0.138 & 0.166 & 0.193 \\
0.45 & 0.051 & 0.076 & 0.101 & 0.127 & 0.152 & 0.177 \\
0.50 & 0.046 & 0.069 & 0.092 & 0.115 & 0.138 & 0.161 \\
0.55 & 0.041 & 0.062 & 0.083 & 0.104 & 0.124 & 0.145 \\
0.60 & 0.037 & 0.055 & 0.074 & 0.092 & 0.111 & 0.129 \\
0.65 & 0.032 & 0.048 & 0.065 & 0.081 & 0.097 & 0.113 \\
0.70 & 0.028 & 0.042 & 0.816 & 0.799 & 0.784 & 0.772 \\
0.75 & 0.023 & 0.856 & 0.847 & 0.842 & 0.842 & 0.846 \\
0.80 & 0.019 & 0.871 & 0.870 & 0.873 & 0.879 & 0.890 \\
0.85 & 0.014 & 0.882 & 0.886 & 0.894 & 0.905 & 0.920 \\
0.90 & 0.009 & 0.890 & 0.898 & 0.908 & 0.922 & 0.939 \\
0.95 & 0.005 & 0.894 & 0.904 & 0.916 & 0.931 & 0.949 \\
\hline
\end{tabular}


Table 5.2.6

Steam/Water $2-\phi$ Flow Cases
(DC Motor, $p_{1}=3$ atm)
(continued)

Normalized Relative Velocity of steam at Vane Exit vs. Inlet Void Fraction for Various Homologous Flow Parameters at $\mathrm{PI}=3.0$ atm. DC Motor Operation

\begin{tabular}{|l|l|l|l|l|l|l|}
\hline \multirow{2}{*}{$\begin{array}{l}\text { Inlet } \\
\text { Void } \\
\text { Fraction }\end{array}$} & \multicolumn{6}{|c|}{ Homologous Flow Parameter } \\
\cline { 2 - 7 } & 0.4 & 0.6 & 0.8 & 1.0 & 1.2 & $\cdots 1.4$ \\
\hline 0.00 & 0.000 & 0.000 & 0.000 & 0.000 & 0.000 & 0.000 \\
0.05 & 0.000 & 0.000 & 0.000 & 0.000 & 0.000 & 0.000 \\
0.10 & 0.000 & 0.000 & 0.000 & 0.000 & 0.000 & 0.000 \\
0.15 & 0.000 & 0.000 & 0.000 & 0.000 & 0.000 & 0.000 \\
0.20 & 0.000 & 0.000 & 0.000 & 0.000 & 0.000 & 0.000 \\
0.25 & 0.000 & 0.000 & 0.000 & 0.000 & 0.000 & 0.000 \\
0.30 & 0.000 & 0.000 & 0.000 & 0.000 & 0.000 & 0.000 \\
0.45 & 0.000 & 0.000 & 0.000 & 0.000 & 0.000 & 0.000 \\
0.50 & 0.000 & 0.000 & 0.000 & 0.000 & 0.000 & 0.000 \\
0.55 & 0.000 & 0.000 & 0.000 & 0.000 & 0.000 & 0.000 \\
0.60 & 0.000 & 0.000 & 0.000 & 0.000 & 0.000 & 0.000 \\
0.65 & 0.000 & 0.000 & 0.000 & 0.000 & 1.000 & 0.000 \\
0.70 & 0.000 & 0.000 & 0.118 & 0.139 & 0.155 & 0.163 \\
0.75 & 0.000 & 0.102 & 0.136 & 0.168 & 0.199 & 0.227 \\
0.80 & 0.000 & 0.111 & 0.149 & 0.187 & 0.225 & 0.262 \\
0.85 & 0.000 & 0.119 & 0.159 & 0.201 & 0.242 & 0.284 \\
0.90 & 0.000 & 0.126 & 0.169 & 0.212 & 0.256 & 0.300 \\
0.95 & 0.000 & 0.132 & 0.177 & 0.222 & 0.267 & 0.313 \\
\hline
\end{tabular}


Table 5.2.6

Steam/Water 2- $\phi$ Flow Cases (DC Motor, $\left.p_{1}=3 \mathrm{~atm}\right)$

(continued)

Discharge Void Fraction vs. Inlet Void Fraction for Various Homologous Flow Parameters at P1 $=3.0 \mathrm{~atm}$. DC Motor Operation

\begin{tabular}{|l|l|l|l|l|l|l|}
\hline \multirow{2}{*}{$\begin{array}{l}\text { Inlet } \\
\text { Void } \\
\text { Fraction }\end{array}$} & \multicolumn{6}{|c|}{ Homologous Flow Parameter } \\
\cline { 2 - 7 } & 0.4 & 0.6 & 0.8 & 1.0 & 1.2 & 1.4 \\
\hline 0.00 & 0.000 & 0.000 & 0.000 & 0.000 & 0.000 & 0.000 \\
0.05 & 0.000 & 0.000 & 0.000 & 0.000 & 0.000 & 0.000 \\
0.10 & 0.000 & 0.000 & 0.000 & 0.000 & 0.000 & 0.000 \\
0.15 & 0.000 & 0.000 & 0.000 & 0.000 & 0.000 & 0.000 \\
0.20 & 0.000 & 0.000 & 0.000 & 0.000 & 0.000 & 0.000 \\
0.25 & 0.000 & 0.000 & 0.000 & 0.000 & 0.000 & 0.000 \\
0.30 & 0.000 & 0.000 & 0.000 & 0.000 & 0.000 & 0.000 \\
0.45 & 0.000 & 0.000 & 0.000 & 0.000 & 0.000 & 0.000 \\
0.50 & 0.000 & 0.000 & 0.000 & 0.000 & 0.000 & 0.000 \\
0.55 & 0.000 & 0.000 & 0.000 & 0.000 & 0.000 & 0.000 \\
0.60 & 0.000 & 0.000 & 0.000 & 0.000 & 0.000 & 0.000 \\
0.65 & 0.000 & 0.000 & 0.000 & 0.000 & 0.000 & 0.000 \\
0.70 & 0.000 & 0.000 & 0.932 & 0.914 & 0.894 & 0.875 \\
0.75 & 0.000 & 0.960 & 0.946 & 0.932 & 0.918 & 0.905 \\
0.80 & 0.000 & 0.968 & 0.958 & 0.947 & 0.937 & 0.928 \\
0.85 & 0.000 & 0.977 & 0.969 & 0.961 & 0.954 & 0.948 \\
0.90 & 0.000 & 0.985 & 0.980 & 0.975 & 0.970 & 0.966 \\
0.95 & 0.000 & 0.992 & 0.990 & 0.987 & 0.985 & 0.983 \\
\hline
\end{tabular}


Table 5.2.7

Steam/Water 2- $\phi$ Flow Cases (DC Motor, $\left.p_{1}=2 \mathrm{~atm}\right)$

Homologous Head Parameter vs. Inlet Void Fraction for Various Homologous Flow Parameters at $P 1=2.0$ atm.

DC Motor Operation

\begin{tabular}{|l|r|r|r|r|r|r|}
\hline \multirow{2}{*}{$\begin{array}{l}\text { Inlet } \\
\text { Void } \\
\text { Fraction }\end{array}$} & \multicolumn{7}{|c|}{ Homologous Flow Parameter } \\
\cline { 2 - 6 } & 0.4 & 0.6 & 0.8 & \multicolumn{1}{|c|}{1.0} & 1.2 & \multicolumn{1}{c|}{1.4} \\
\hline 0.00 & 1.008 & 1.005 & 1.002 & 1.000 & 0.999 & 0.999 \\
0.05 & 1.013 & 1.265 & 1.611 & 1.615 & 1.700 & 1.673 \\
0.10 & 1.019 & 1.365 & 1.495 & 1.623 & 1.458 & 1.605 \\
0.15 & 1.025 & 1.028 & 1.676 & 1.486 & 2.032 & 1.572 \\
0.20 & 1.030 & 0.945 & 1.397 & 1.488 & 1.458 & 1.550 \\
0.25 & 1.036 & 0.880 & 1.318 & 1.452 & 1.412 & 1.430 \\
0.30 & 1.042 & 0.899 & 1.006 & 0.889 & 1.310 & 1.283 \\
0.35 & 1.048 & 0.987 & 0.847 & 0.776 & 0.741 & 0.760 \\
0.40 & 1.054 & 0.979 & 0.804 & 0.718 & 0.677 & 0.635 \\
0.45 & 1.059 & 0.968 & 0.757 & 0.622 & 0.565 & 0.522 \\
0.50 & 1.065 & 0.954 & 0.704 & 0.527 & 0.431 & 0.373 \\
0.55 & 1.071 & 0.935 & 0.639 & 0.416 & 0.279 & 0.175 \\
0.60 & 1.076 & 0.916 & 0.560 & 0.282 & 0.088 & -0.039 \\
0.65 & 1.082 & 0.890 & 0.458 & 0.112 & -0.143 & -0.334 \\
0.70 & 1.087 & 0.856 & -0.693 & -1.139 & -0.479 & -0.722 \\
0.75 & 1.093 & -0.244 & -0.379 & -0.572 & -0.827 & -1.150 \\
0.80 & 1.098 & -0.143 & -0.190 & -0.266 & -0.366 & -0.487 \\
0.85 & 1.103 & -0.069 & -0.075 & -0.092 & -0.121 & -0.160 \\
0.90 & 1.108 & 0.000 & 0.013 & 0.020 & 0.021 & 0.014 \\
0.95 & 1.135 & 0.172 & 0.182 & 0.185 & 0.203 & 0.213 \\
\hline
\end{tabular}


Table 5.2.7

Steam/Water 2- $\phi$ Flow Cases

(DC Motor, $p_{1}=2 \mathrm{~atm}$ )

(continued)

Static Pressure Rise in psia at Vane Exit vs. Inlet Void Fraction for Various Homologous Flow Parameters at $\mathrm{PI}=2.0 \mathrm{~atm}$.

DC Motor Operation

\begin{tabular}{|l|r|r|r|r|r|r|}
\hline \multirow{2}{*}{$\begin{array}{l}\text { Inlet } \\
\text { Void } \\
\text { Fraction }\end{array}$} & \multicolumn{7}{|c|}{ Homologous Flow Parameter } \\
\cline { 2 - 7 } & 0.4 & 0.6 & 0.8 & 1.0 & 1.2 & 1.4 \\
\hline 0.00 & 14.79 & 14.84 & 14.91 & 14.99 & 1.5 .10 & 15.22 \\
0.05 & 16.34 & 23.05 & 31.71 & 31.32 & 32.81 & 31.61 \\
0.10 & 18.07 & 27.21 & 30.22 & 32.93 & 28.51 & 31.44 \\
0.15 & 20.00 & 20.03 & 36.50 & 31.19 & 43.58 & 32.23 \\
0.20 & 22.17 & 19.80 & 31.30 & 33.08 & 31.89 & 33.50 \\
0.25 & 24.63 & 20.31 & 31.47 & 34.34 & 32.86 & 32.84 \\
0.30 & 27.43 & 23.41 & 26.05 & 23.01 & 32.93 & 31.92 \\
0.35 & 30.67 & 28.73 & 24.96 & 23.14 & 22.37 & 22.95 \\
0.40 & 34.44 & 32.07 & 27.33 & 25.15 & 24.25 & 23.46 \\
0.45 & 38.89 & 36.02 & 30.30 & 26.92 & 25.68 & 24.92 \\
0.50 & 44.23 & 40.76 & 33.99 & 29.56 & 27.45 & 26.44 \\
0.55 & 50.74 & 46.56 & 38.57 & 33.00 & 29.97 & 28.03 \\
0.60 & 58.87 & 53.96 & 44.41 & 37.51 & 33.20 & 30.88 \\
0.65 & 69.32 & 63.50 & 51.97 & 43.42 & 37.79 & 34.18 \\
0.70 & 83.24 & 76.29 & 0.34 & 0.55 & 43.38 & 38.86 \\
0.75 & 102.68 & 0.11 & 0.19 & 0.28 & 0.39 & 0.52 \\
0.80 & 131.79 & 0.06 & 0.10 & 0.14 & 0.19 & 0.24 \\
0.85 & 180.14 & 0.04 & 0.05 & 0.07 & 0.09 & 0.10 \\
0.90 & 276.29 & 0.02 & 0.03 & 0.03 & 0.04 & 0.04 \\
0.95 & 560.75 & 0.02 & 0.02 & 0.02 & 0.02 & 0.02 \\
\hline
\end{tabular}


Table 5.2.7

Steam/Water 2- $\phi$ Flow Cases (DC Motor, $\left.p_{1}=2 \mathrm{~atm}\right)$

(continued)

Normalized Relative Velocity of Water at Vane Exit vs. Inlet Void Fraction for Various Homologous Flow Parameters at $P 1=2.0$ atm. DC Motor Operation

\begin{tabular}{|l|l|l|l|l|l|l|}
\hline \multirow{2}{*}{$\begin{array}{l}\text { Inlet } \\
\text { Void } \\
\text { Fraction }\end{array}$} & \multicolumn{5}{|c|}{ Homologous Flow Parameter } \\
\cline { 2 - 7 } & 0.4 & 0.6 & 0.8 & 1.0 & 1.2 & 1.4 \\
\hline 0.00 & 0.092 & 0.138 & 0.184 & 0.230 & 0.276 & 0.322 \\
0.05 & 0.087 & 0.131 & 0.175 & 0.218 & 0.262 & 0.306 \\
0.10 & 0.083 & 0.124 & 0.166 & 0.207 & 0.248 & 0.290 \\
0.15 & 0.078 & 0.117 & 0.156 & 0.195 & 0.234 & 0.273 \\
0.20 & 0.073 & 0.110 & 0.147 & 0.184 & 0.221 & 0.257 \\
0.25 & 0.069 & 0.103 & 0.138 & 0.173 & 0.207 & 0.241 \\
0.30 & 0.064 & 0.097 & 0.129 & 0.161 & 0.193 & 0.225 \\
0.35 & 0.060 & 0.090 & 0.120 & 0.150 & 0.179 & 0.209 \\
0.40 & 0.055 & 0.083 & 0.110 & 0.138 & 0.166 & 0.193 \\
0.45 & 0.051 & 0.076 & 0.101 & 0.126 & 0.152 & 0.177 \\
0.50 & 0.046 & 0.069 & 0.092 & 0.115 & 0.138 & 0.161 \\
0.55 & 0.041 & 0.062 & 0.083 & 0.104 & 0.124 & 0.145 \\
0.60 & 0.037 & 0.055 & 0.074 & 0.092 & 0.110 & 0.129 \\
0.65 & 0.032 & 0.048 & 0.065 & 0.081 & 0.097 & 0.113 \\
0.70 & 0.028 & 0.042 & 0.812 & 0.787 & 0.083 & 0.097 \\
0.75 & 0.023 & 0.856 & 0.847 & 0.840 & 0.836 & 0.834 \\
0.80 & 0.019 & 0.872 & 0.871 & 0.873 & 0.879 & 0.889 \\
0.85 & 0.014 & 0.883 & 0.888 & 0.895 & 0.906 & 0.920 \\
0.90 & 0.009 & 0.891 & 0.899 & 0.909 & 0.923 & 0.940 \\
0.95 & 0.005 & 0.895 & 0.905 & 0.918 & 0.933 & 0.950 \\
\hline
\end{tabular}


Table 5.2.7

Steam/Water 2- $\phi$ Flow Cases

(DC Motor, $p_{1}=2 \mathrm{~atm}$ )

(continued)

Normalized Relative Velocity of steam at Vane Exit vs. Inlet Void Fraction for Various Homologous Flow Parameters at $P 1=2.0$ atm.

DC Motor Operation

\begin{tabular}{|l|l|l|l|l|l|l|}
\hline \multirow{2}{*}{$\begin{array}{l}\text { Inlet } \\
\text { Void } \\
\text { Fraction }\end{array}$} & \multicolumn{6}{|c|}{ Homologous Flow Parameter } \\
\cline { 2 - 7 } & 0.4 & 0.6 & 0.8 & 1.0 & 1.2 & 1.4 \\
\hline 0.00 & 0.000 & 0.000 & 0.000 & 0.000 & 0.000 & 0.000 \\
0.05 & 0.000 & 0.000 & 0.000 & 0.000 & 0.000 & 0.000 \\
0.10 & 0.000 & 0.000 & 0.000 & 0.000 & 0.000 & 0.000 \\
0.15 & 0.000 & 0.000 & 0.000 & 0.000 & 0.000 & 0.000 \\
0.20 & 0.000 & 0.000 & 0.000 & 0.000 & 0.000 & 0.000 \\
0.25 & 0.000 & 0.000 & 0.000 & 0.000 & 0.000 & 0.000 \\
0.30 & 0.000 & 0.000 & 0.000 & 0.000 & 0.000 & 0.000 \\
0.45 & 0.000 & 0.000 & 0.000 & 0.000 & 0.000 & 0.000 \\
0.50 & 0.000 & 0.000 & 0.000 & 0.000 & 0.000 & 0.000 \\
0.55 & 0.000 & 0.000 & 0.000 & 0.000 & 0.000 & 0.000 \\
0.60 & 0.000 & 0.000 & 0.000 & 0.000 & 0.000 & 0.000 \\
0.65 & 0.000 & 0.000 & 0.000 & 0.000 & 0.000 & 0.000 \\
0.70 & 0.000 & 0.000 & 0.099 & 0.098 & 0.000 & 0.000 \\
0.75 & 0.000 & 0.098 & 0.127 & 0.151 & 0.169 & 0.180 \\
0.80 & 0.000 & 0.109 & 0.145 & 0.180 & 0.214 & 0.247 \\
0.85 & 0.000 & 0.118 & 0.158 & 0.199 & 0.239 & 0.279 \\
0.90 & 0.000 & 0.126 & 0.168 & 0.211 & 0.255 & 0.299 \\
0.95 & 0.000 & 0.132 & 0.177 & 0.222 & 0.267 & 0.312 \\
\hline
\end{tabular}


Table 5.2.7

Steam/Water 2- $\phi$ Flow Cases

(DC Motor, $p_{1}=2 \mathrm{~atm}$ )

(continued)

Discharge Void Fraction vs. Inlet Void Fraction for Varlous Homologous Flow Parameters at $P 1=2.0 \mathrm{~atm}$. DC Motor Operation

\begin{tabular}{|l|l|l|l|l|l|l|}
\hline \multirow{2}{*}{$\begin{array}{l}\text { Inlet } \\
\text { Vold } \\
\text { Fraction }\end{array}$} & \multicolumn{6}{|c|}{ Homologous Flow Parameter } \\
\cline { 2 - 7 } & 0.4 & 0.6 & 0.8 & 1.0 & 1.2 & 1.4 \\
\hline 0.00 & 0.000 & 0.000 & 0.000 & 0.000 & 0.000 & .0 .000 \\
0.05 & 0.000 & 0.000 & 0.000 & 0.000 & 0.000 & 0.000 \\
0.10 & 0.000 & 0.000 & 0.000 & 0.000 & 0.000 & 0.000 \\
0.15 & 0.000 & 0.000 & 0.000 & 0.000 & 0.000 & 0.000 \\
0.20 & 0.000 & 0.000 & 0.000 & 0.000 & 0.000 & 0.000 \\
0.25 & 0.000 & 0.000 & 0.000 & 0.000 & 0.000 & 0.000 \\
0.30 & 0.000 & 0.000 & 0.000 & 0.000 & 0.000 & 0.000 \\
0.45 & 0.000 & 0.000 & 0.000 & 0.000 & 0.000 & 0.000 \\
0.50 & 0.000 & 0.000 & 0.000 & 0.000 & 0.000 & 0.000 \\
0.55 & 0.000 & 0.000 & 0.000 & 0.000 & 0.000 & 0.000 \\
0.60 & 0.000 & 0.000 & 0.000 & 0.000 & 0.000 & 0.000 \\
0.65 & 0.000 & 0.000 & 0.000 & 0.000 & 0.000 & 0.000 \\
0.70 & 0.000 & 0.000 & 0.932 & 0.912 & 0.000 & 0.000 \\
0.75 & 0.000 & 0.960 & 0.946 & 0.932 & 0.917 & 0.903 \\
0.80 & 0.000 & 0.968 & 0.958 & 0.947 & 0.937 & 0.928 \\
0.85 & 0.000 & 0.977 & 0.969 & 0.961 & 0.954 & 0.948 \\
0.90 & 0.000 & 0.985 & 0.980 & 0.975 & 0.970 & 0.966 \\
0.95 & 0.000 & 0.992 & 0.990 & 0.987 & 0.985 & 0.983 \\
\hline
\end{tabular}


Table $\mathbf{5 . 2 . 8}$

Steam/Water 2- $\phi$ Flow Cases (DC Motor, $p_{1}=1 \mathrm{~atm}$ )

Homologous Head Parameter vs. Inlet Vold Fraction for Various Homologous Flow Parameters at $P_{1}=1.0 \mathrm{~atm}$. DC Motor Operation

\begin{tabular}{|l|r|r|r|r|r|r|}
\hline \multirow{2}{*}{$\begin{array}{l}\text { Inlet } \\
\text { Vold } \\
\text { Fraction }\end{array}$} & \multicolumn{7}{|c|}{ Homologous Flow Parameter } \\
\cline { 2 - 7 } & 0.4 & 0.6 & 0.8 & 1.0 & 1.2 & 1.4 \\
\hline 0.00 & 1.008 & 1.005 & 1.002 & 1.000 & 0.999 & 0.999 \\
0.05 & 1.249 & 1.289 & 1.302 & 1.319 & 1.286 & 1.344 \\
0.10 & 1.244 & 1.272 & 1.259 & 1.279 & 1.313 & 1.325 \\
0.15 & 1.268 & 1.237 & 1.253 & 1.267 & 1.293 & 1.307 \\
0.20 & 1.260 & 1.238 & 1.224 & 1.231 & 1.267 & 1.285 \\
0.25 & 1.261 & 1.151 & 1.248 & 1.218 & 1.241 & 1.251 \\
0.30 & 1.042 & 1.052 & 1.172 & 1.170 & 1.194 & 1.222 \\
0.35 & 1.047 & 1.014 & 0.985 & 0.986 & 1.024 & 1.071 \\
0.40 & 1.053 & 1.015 & 0.970 & 0.966 & 0.992 & 1.026 \\
0.45 & 1.059 & 1.007 & 0.944 & 0.927 & 0.928 & 0.989 \\
0.50 & 1.065 & 0.992 & 0.902 & 0.858 & 0.852 & 0.916 \\
0.55 & 1.071 & 0.982 & 0.857 & 0.803 & 0.779 & 0.769 \\
0.60 & 1.076 & 0.966 & 0.810 & 0.730 & 0.683 & 0.658 \\
0.65 & 1.082 & 0.946 & 0.749 & 0.625 & 0.550 & 0.521 \\
0.70 & 1.088 & 0.922 & 0.671 & 0.302 & 0.394 & 0.313 \\
0.75 & 1.093 & 0.389 & 0.551 & 0.319 & 0.160 & 0.048 \\
0.80 & 1.099 & -0.189 & -0.319 & -0.507 & -0.761 & -1.103 \\
0.85 & 1.104 & -0.083 & -0.122 & -0.181 & -0.257 & -0.349 \\
0.90 & 1.109 & -0.017 & -0.017 & -0.025 & -0.039 & -0.061 \\
0.95 & 1.124 & 0.058 & 0.070 & 0.084 & 0.094 & 0.093 \\
\hline
\end{tabular}


Table 5.2.8

Steam/Water 2- $\phi$ Flow Cases (DC Motor, $p_{1}=1 \mathrm{~atm}$ )

(contlnued)

Static Pressure Rise in psia at Vane Exit ve. Inlet Vold Fraction for Varlous Homologous Flow Parameters at FI = $1.0 \mathrm{~atm}$.

DC Motor Operation

\begin{tabular}{|l|r|r|r|r|r|r|}
\hline \multirow{2}{*}{$\begin{array}{l}\text { Inlat } \\
\text { Vold } \\
\text { Fract1on }\end{array}$} & \multicolumn{7}{|c|}{ Homologous Flow Parameter } \\
\cline { 2 - 7 } & 0.4 & 0.6 & 0.8 & 1.0 & 1.2 & 1.4 \\
\hline 0.00 & 15.04 & 15.09 & 15.16 & 15.25 & 15.36 & 15.49 \\
0.05 & 22.37 & 23.30 & 23.45 & 23.68 & 22.67 & 23.82 \\
0.10 & 22.93 & 23.49 & 22.94 & 23.24 & 23.83 & 23.86 \\
0.15 & 21.63 & 23.30 & 23.48 & 23.58 & 23.97 & 24.03 \\
0.20 & 25.14 & 24.20 & 23.56 & 23.45 & 24.06 & 24.21 \\
0.25 & 26.24 & 22.88 & 25.12 & 24.03 & 24.31 & 24.25 \\
0.30 & 21.41 & 21.41 & 24.29 & 23.91 & 24.20 & 24.57 \\
0.35 & 23.03 & 21.79 & 20.78 & 20.60 & 21.38 & 22.31 \\
0.40 & 24.92 & 23.49 & 21.99 & 21.65 & 22.09 & 22.72 \\
0.45 & 27.16 & 25.27 & 23.26 & 22.57 & 22.39 & 23.64 \\
0.50 & 29.84 & 27.33 & 24.56 & 23.17 & 22.84 & 24.20 \\
0.55 & 33.10 & 30.11 & 26.37 & 24.71 & 23.96 & 23.63 \\
0.60 & 37.18 & 33.52 & 28.94 & 26.60 & 25.31 & 24.67 \\
0.65 & 42.42 & 38.00 & 32.31 & 28.88 & 26.95 & 26.30 \\
0.70 & 49.40 & 44.10 & 36.97 & 32.41 & 29.75 & 28.01 \\
0.75 & 59.17 & 52.76 & 43.31 & 37.25 & 33.48 & 31.18 \\
0.80 & 73.79 & 0.05 & 0.09 & 0.14 & 0.20 & 0.28 \\
0.85 & 98.13 & 0.03 & 0.04 & 0.06 & 0.08 & 0.10 \\
0.90 & 146.66 & 0.01 & 0.02 & 0.02 & 0.03 & 0.03 \\
0.95 & 291.39 & 0.01 & 0.01 & 0.01 & 0.01 & 0.01 \\
\hline
\end{tabular}


Table $\mathbf{5 . 2 . 8}$

Steam/Water 2- $\phi$ Flow Cases
(DC Motor, $p_{1}=1$ atm)
(contlaued)

Normalized Relative Velocity of Water at Vane Exit vs. Inlet Void Fraction for Varlous Homologous Flow parameters at $P 1=1.0$ atm. DC Motor Operation

\begin{tabular}{|l|l|l|l|l|l|l|}
\hline \multirow{2}{*}{$\begin{array}{l}\text { Inlet } \\
\text { Vold } \\
\text { Fraction }\end{array}$} & \multicolumn{5}{|c|}{ Homologous Flow Parameter } \\
\cline { 2 - 7 } & 0.4 & 0.6 & 0.8 & 1.0 & 1.2 & 1.4 \\
\hline 0.00 & 0.092 & 0.138 & 0.184 & 0.230 & 0.276 & 0.322 \\
0.05 & 0.087 & 0.131 & 0.175 & 0.218 & 0.262 & 0.306 \\
0.10 & 0.083 & 0.124 & 0.166 & 0.207 & 0.248 & 0.290 \\
0.15 & 0.078 & 0.117 & 0.156 & 0.195 & 0.234 & 0.273 \\
0.20 & 0.073 & 0.110 & 0.147 & 0.184 & 0.221 & 0.257 \\
0.25 & 0.069 & 0.103 & 0.138 & 0.172 & 0.207 & 0.241 \\
0.30 & 0.064 & 0.097 & 0.129 & 0.161 & 0.193 & 0.225 \\
0.35 & 0.060 & 0.090 & 0.120 & 0.150 & 0.179 & 0.209 \\
0.40 & 0.055 & 0.083 & 0.110 & 0.138 & 0.166 & 0.193 \\
0.45 & 0.051 & 0.076 & 0.101 & 0.126 & 0.152 & 0.177 \\
0.50 & 0.046 & 0.069 & 0.092 & 0.115 & 0.138 & 0.161 \\
0.55 & 0.041 & 0.062 & 0.083 & 0.103 & 0.124 & 0.145 \\
0.60 & 0.037 & 0.055 & 0.074 & 0.092 & 0.110 & 0.129 \\
0.65 & 0.032 & 0.048 & 0.064 & 0.080 & 0.097 & 0.113 \\
0.70 & 0.028 & 0.041 & 0.055 & 0.069 & 0.083 & 0.097 \\
0.75 & 0.023 & 0.035 & 0.046 & 0.058 & 0.069 & 0.081 \\
0.80 & 0.018 & 0.871 & 0.869 & 0.868 & 0.870 & 0.872 \\
0.85 & 0.014 & 0.883 & 0.887 & 0.894 & 0.904 & 0.918 \\
0.90 & 0.009 & 0.890 & 0.898 & 0.909 & 0.922 & 0.939 \\
0.95 & 0.005 & 0.895 & 0.905 & 0.917 & 0.932 & 0.950 \\
\hline
\end{tabular}


Table 5.2.8 Steam/Water 2- $\phi$ Flow Cases (DC Motor, $p_{1}=1 \mathrm{~atm}$ )

(contlaued)

Normalized Relative Velocity of steam at Vane Exit vs. Inlet Void Fraction for Various Homologous Flow Parameters at P1 $=1.0$ atm. DC Motor Operation

\begin{tabular}{|l|l|l|l|l|l|l|}
\hline \multirow{2}{*}{$\begin{array}{l}\text { Inlet } \\
\text { Vold } \\
\text { Fraction }\end{array}$} & \multicolumn{5}{|c|}{ Homologous Flow Parameter } \\
\cline { 2 - 7 } & 0.4 & 0.6 & 0.8 & 1.0 & 1.2 & 1.4 \\
\hline 0.00 & 0.000 & 0.000 & 0.000 & 0.000 & 0.000 & 0.000 \\
0.05 & 0.000 & 0.000 & 0.000 & 0.000 & 0.000 & 0.000 \\
0.10 & 0.000 & 0.000 & 0.000 & 0.000 & 0.000 & 0.000 \\
0.15 & 0.000 & 0.000 & 0.000 & 0.000 & 0.000 & 0.000 \\
0.20 & 0.000 & 0.000 & 0.000 & 0.000 & 0.000 & 0.000 \\
0.25 & 0.000 & 0.000 & 0.000 & 0.000 & 0.000 & 0.000 \\
0.30 & 0.000 & 0.000 & 0.000 & 0.000 & 0.000 & 0.000 \\
0.45 & 0.000 & 0.000 & 0.000 & 0.000 & 0.000 & 0.000 \\
0.50 & 0.000 & 0.000 & 0.000 & 0.000 & 0.000 & 0.000 \\
0.55 & 0.000 & 0.000 & 0.000 & 0.000 & 0.000 & 0.000 \\
0.60 & 0.000 & 0.000 & 0.000 & 0.000 & 0.000 & 0.000 \\
0.65 & 0.000 & 0.000 & 0.000 & 0.000 & 0.000 & 0.000 \\
0.70 & 0.000 & 0.000 & 0.000 & 0.000 & 0.000 & 0.000 \\
0.75 & 0.000 & 0.000 & 0.000 & 0.000 & 0.000 & 0.000 \\
0.80 & 0.000 & 0.102 & 0.129 & 0.147 & 0.155 & 0.149 \\
0.85 & 0.000 & 0.125 & 0.153 & 0.289 & 0.223 & 0.255 \\
0.90 & 0.000 & 0.125 & 0.167 & 0.209 & 0.251 & 0.294 \\
0.95 & 0.000 & 0.132 & 0.177 & 0.221 & 0.267 & 0.312 \\
\hline
\end{tabular}


Table 5.2 .8

Steam/Water 2- $\phi$ Flow Cases

(DC Motor, $p_{1}=1 \mathrm{~atm}$ )

(continued)

Discharge Void Fraction Vs. Inlet Void Fraction for Various Homologous Flow Parameters at P1 $=1.0$ atm. DC Motor Operation

\begin{tabular}{|l|l|l|l|l|l|l|}
\hline \multirow{2}{*}{$\begin{array}{l}\text { Inlet } \\
\text { Vold } \\
\text { Fraction }\end{array}$} & \multicolumn{6}{|c|}{ Homologous Flow Parameter } \\
\cline { 2 - 6 } & 0.4 & 0.6 & 0.8 & 1.0 & 1.2 & 1.4 \\
\hline 0.00 & 0.000 & 0.000 & 0.000 & 0.000 & 0.000 & .0 .000 \\
0.05 & 0.000 & 0.000 & 0.000 & 0.000 & 0.000 & 0.000 \\
0.10 & 0.000 & 0.000 & 0.000 & 0.000 & 0.000 & 0.000 \\
0.15 & 0.000 & 0.000 & 0.000 & 0.000 & 0.000 & 0.000 \\
0.20 & 0.000 & 0.000 & 0.000 & 0.000 & 0.000 & 0.000 \\
0.25 & 0.000 & 0.000 & 0.000 & 0.000 & 0.000 & 0.000 \\
0.30 & 0.000 & 0.000 & 0.000 & 0.000 & 0.000 & 0.000 \\
0.45 & 0.000 & 0.000 & 0.000 & 0.000 & 0.000 & 0.000 \\
0.50 & 0.000 & 0.000 & 0.000 & 0.000 & 0.000 & 0.000 \\
0.55 & 0.000 & 0.000 & 0.000 & 0.000 & 0.000 & 0.000 \\
0.60 & 0.000 & 0.000 & 0.000 & 0.000 & 0.000 & 0.000 \\
0.65 & 0.000 & 0.000 & 0.000 & 0.000 & 0.000 & 0.000 \\
0.70 & 0.000 & 0.000 & 0.000 & 0.000 & 0.000 & 0.000 \\
0.75 & 0.000 & 0.000 & 0.000 & 0.000 & 0.000 & 0.000 \\
0.80 & 0.000 & 0.968 & 0.958 & 0.947 & 0.937 & 0.926 \\
0.85 & 0.000 & 0.977 & 0.969 & 0.961 & 0.954 & 0.947 \\
0.90 & 0.000 & 0.985 & 0.980 & 0.975 & 0.970 & 0.966 \\
0.95 & 0.000 & 0.992 & 0.990 & 0.987 & 0.985 & 0.983 \\
\hline
\end{tabular}


Table 5.2.9

Steam/Water 2- $\phi$ Flow Cases

(DC Motor, $p_{1}=0.05 \mathrm{~atm}$ )

Homologous Head Parameter vs. Inlet Void Fraction

for Various Homologous Flow Parameters at P1 $=0.05 \mathrm{~atm}$.

DC Motor Operation

\begin{tabular}{|l|l|l|r|r|r|r|}
\hline \multirow{2}{*}{$\begin{array}{l}\text { Inlet } \\
\text { Void } \\
\text { Fraction }\end{array}$} & \multicolumn{7}{|c|}{ Homologous Flow Parameter } \\
\cline { 2 - 7 } & 0.4 & 0.6 & 0.8 & 1.0 & 1.2 & 1.4 \\
\hline 0.00 & 1.008 & 1.005 & 1.002 & 1.000 & 0.999 & 0.999 \\
0.05 & 1.025 & 1.026 & 1.026 & 0.945 & 1.032 & 1.037 \\
0.10 & 1.029 & 1.033 & 1.037 & 1.024 & 1.051 & 1.061 \\
0.15 & 1.033 & 1.040 & 1.048 & 1.052 & 1.069 & 1.082 \\
0.20 & 1.038 & 1.047 & 1.058 & 1.070 & 1.086 & 1.104 \\
0.25 & 1.041 & 1.054 & 1.068 & 1.084 & 1.086 & 1.126 \\
0.30 & 1.045 & 1.060 & 1.078 & 1.098 & 1.117 & 1.147 \\
0.35 & & & & & & \\
0.40 & & & & & & \\
0.45 & 1.059 & 1.012 & 1.100 & 1.143 & 1.177 & 1.196 \\
0.50 & 1.065 & 1.093 & 1.124 & 1.157 & 1.159 & 1.231 \\
0.55 & 1.070 & 1.102 & 1.137 & 1.175 & 1.218 & 1.265 \\
0.60 & 1.076 & 1.110 & 1.149 & 1.191 & 1.238 & 1.290 \\
0.65 & 1.082 & 1.116 & 1.160 & 1.205 & 1.257 & 1.313 \\
0.70 & 1.088 & 1.118 & 1.162 & 1.217 & 1.274 & 1.336 \\
0.75 & 1.093 & 1.121 & 1.167 & 1.215 & 1.287 & 1.355 \\
0.80 & 1.098 & 1.117 & 1.161 & 1.214 & 1.273 & 1.354 \\
0.85 & 1.104 & 1.111 & 1.140 & 1.184 & 1.252 & 1.325 \\
0.90 & 1.109 & 1.095 & 1.106 & 1.140 & 1.187 & 1.248 \\
0.95 & 1.115 & -0.019 & -0.034 & -0.061 & -0.100 & -0.153 \\
\hline
\end{tabular}




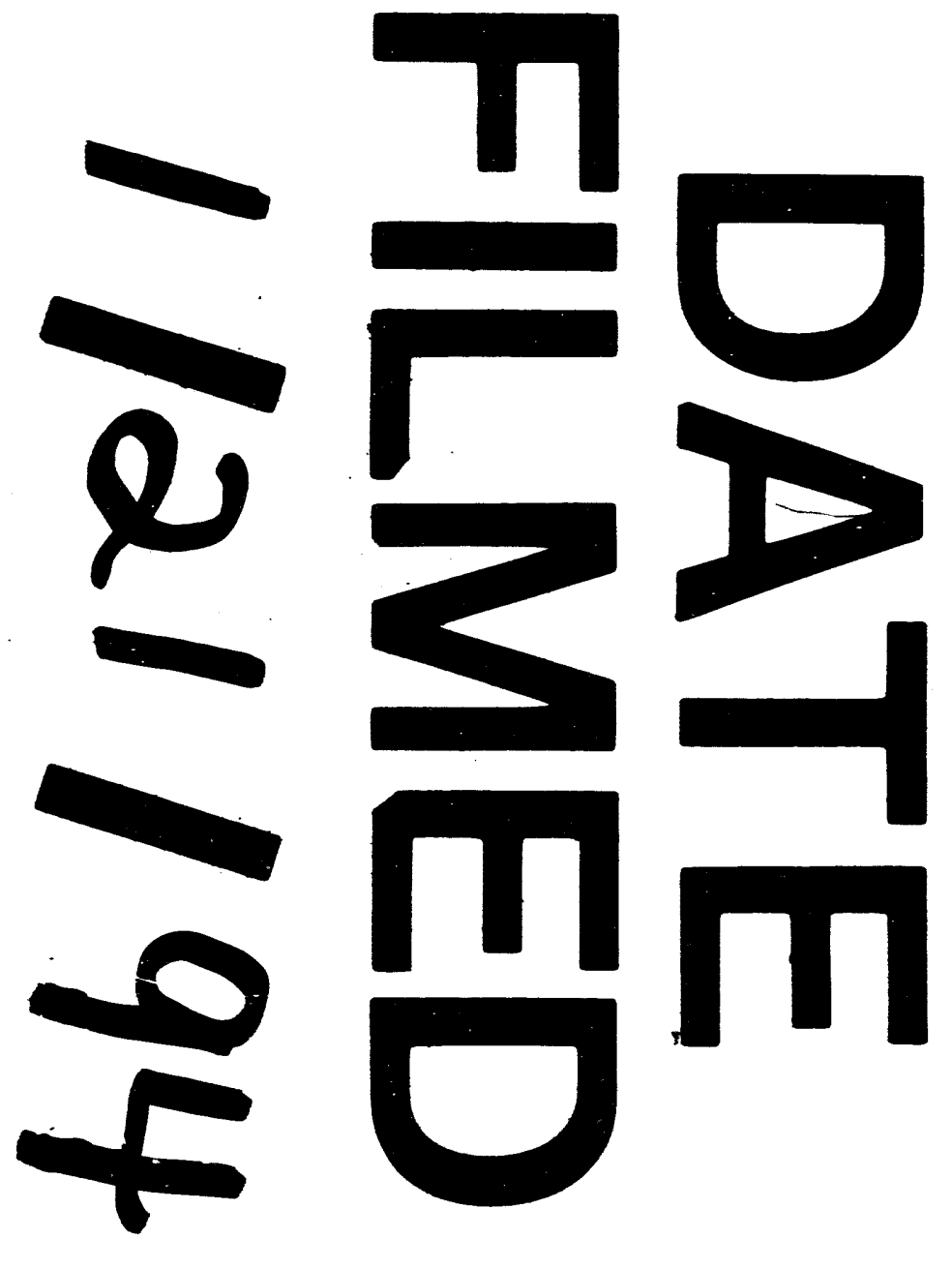


